\title{
Ruthenium-Catalyzed C-H Arylation of Diverse Aryl Carboxylic Acids with Aryl- and Heteroaryl Halides
}

\author{
Liangbin Huang and Daniel J. Weix* \\ Department of Chemistry, University of Rochester, Rochester, NY, USA 14627

\section{Supporting Information}

General Methods

S2-3

Optimization, Control, and Mechanism Experiments

S4-7

General Procedure for the ortho-Arylation and Examples

S8-29

NMR Data

S30-81 


\section{General Informations}

\section{Reagents}

\section{Metals}

$\left[\mathrm{Ru}\left(p \text {-cymene) } \mathrm{Cl}_{2}\right]_{2}\right.$ (Alfa Aesar), $\left[\mathrm{IrCp}^{*} \mathrm{Cl}_{2}\right]_{2}$ (Alfa Aesar) $\mathrm{CoBr}_{2}$ (Aldrich), $\mathrm{CrCl}_{3}(\mathrm{Strem}), \mathrm{Cu}(\mathrm{OAc})_{2}$ (Aldrich), $\mathrm{RuCl}_{3}$ hydrate (Pressure Chemical), were used as received and stored on the bench. ( $p$ cymene) $\mathrm{Ru}\left(\kappa^{2}-\mathrm{O}, \mathrm{C}\right.$-napthenoate)(py) was prepared according to the literature procedure. ${ }^{1}$

\section{Ligand}

4-4'-di-tert-butyl-2,2'-bipyridine (Aldrich) and $\mathrm{PCy}_{3}$ (Aldrich) was used as received. Other ligands tested were from commercial suppliers and used as received.

\section{Solvents}

NMP, anhydrous, 99.5\% (Sigma-Aldrich) was stored in a nitrogen-filled glove box.

\section{Other Reagents}

Dodecane (Aldrich), $\mathrm{K}_{2} \mathrm{CO}_{3}$ (Combi-blocks), $\mathrm{Cs}_{2} \mathrm{CO}_{3}$ (Alfa Aesar), MeI (Aldrich), carboxylic acids and (het)aryl halides (Alfa Aesar, Aldrich, Combi-Blocks, etc.) were used as received.

\section{Methods}

\section{NMR Spectroscopy}

${ }^{1} \mathrm{H}$ nuclear magnetic resonance (NMR) spectroscopy chemical shifts are reported in ppm and referenced to TMS (tetramethylsilane) in $\mathrm{CDCl}_{3}(\delta=0 \mathrm{ppm})$ or the residual solvent peak for $\mathrm{CDCl}_{3}(\delta=7.26 \mathrm{ppm})$. For ${ }^{13} \mathrm{C}$ NMR and ${ }^{19} \mathrm{~F}$ NMR chemical shifts, the residual solvent peak $\left(\mathrm{CDCl}_{3}, \delta=77.00 \mathrm{ppm}\right)$ and the external standard, $\alpha, \alpha, \alpha$-trifluorotoluene $(\delta=0 \mathrm{ppm})$ were used as references. NMR spectra were recorded on Avance Bruker NMR spectrometers operating at either $400.13 \mathrm{MHz}$ or $500.13 \mathrm{MHz}$ and data analysis was performed using the iNMR software package (www.inmr.net). Chemical shifts are reported in parts per million (ppm), multiplicities are indicated by s (singlet), $d$ (doublet), $t$ (triplet), $q$ (quartet), m (multiplet) and br (broad). Coupling constants ( $\mathrm{J})$ are reported in Hertz.

\section{Gas Chromatography}

GC analyses were performed on an Agilent 7890A GC equipped with dual DB-5 columns $(20 \mathrm{~m} \times 180$ $\mu \mathrm{m} \times 0.18 \mu \mathrm{m}$ ), dual FID detectors, and hydrogen as the carrier gas. A sample volume of $1 \mu \mathrm{L}$ was injected at a temperature of $300{ }^{\circ} \mathrm{C}$ and a $100: 1$ split ratio. The initial inlet pressure was 20.3 psi but varied as the column flow was held constant at $1.8 \mathrm{~mL} / \mathrm{min}$ for the duration of the run. The initial oven temperature of $50{ }^{\circ} \mathrm{C}$ was held for $0.46 \mathrm{~min}$ followed by a temperature ramp of $65{ }^{\circ} \mathrm{C} / \mathrm{min}$ up to $300{ }^{\circ} \mathrm{C}$.

\footnotetext{
${ }^{1}$ Warratz, S.; Kornhaaß, C.; Cajaraville, A.; Niepötter, B.; Stalke, D.; Ackermann, L., Angew. Chem. Int. Ed. 2015, 54, 55135517
} 
The temperature was held at $300{ }^{\circ} \mathrm{C}$ for $3 \mathrm{~min}$. The total run time was $\sim 7.3 \mathrm{~min}$ and the FID temperature was $325^{\circ} \mathrm{C}$.

\section{GC/MS Analysis}

GC/MS analyses were performed on a Shimadzu GCMS-QP2010 equipped with an RTX-XLB column $(30 \mathrm{~m} \times 0.25 \mathrm{~mm} \times 0.28 \mu \mathrm{m})$ with a quadrupole mass analyzer using helium as the carrier gas. The analysis method used in all cases was $5 \mu \mathrm{L}$ injection of sample, an injection temp of $225^{\circ} \mathrm{C}$, and a $25: 1$ split ratio. The initial inlet pressure was $7.8 \mathrm{psi}$, but varied as the column flow was held constant at 1.0 $\mathrm{mL} / \mathrm{min}$ for the duration of the run. The interface temperature was held at $250{ }^{\circ} \mathrm{C}$, and the ion source $(\mathrm{EI}+, 30 \mathrm{eV})$ was held at $250^{\circ} \mathrm{C}$. The initial oven temperature was held at $50^{\circ} \mathrm{C}$ for 2 min with the detector off, followed by a temperature ramp, with the detector on, to $280{ }^{\circ} \mathrm{C}$ at $40^{\circ} \mathrm{C} / \mathrm{min}$. The temperature was held at $280{ }^{\circ} \mathrm{C}$ for $3 \mathrm{~min}$. Total run time was $11.75 \mathrm{~min}$.

\section{Low Resolution Mass Spectrometry (LRMS) Analysis}

LRMS analyses were performed on a Shimadzu LCMS-2010A equipped with an ESI or ACPI probe with a quadrupole mass analyzer. Direct injection analysis was employed in all cases with $5 \mu \mathrm{L}$ of sample solution in methanol. The ion source (electron spray ionization, ESI) was held at $250{ }^{\circ} \mathrm{C}$ or $400{ }^{\circ} \mathrm{C}$ when atmospheric-pressure chemical ionization (APCI) was used, sample flow rate at $1 \mathrm{~mL} / \mathrm{min}$.

\section{Infrared Spectroscopy}

Infrared (IR) spectra were recorded on a Shimadzu IRAffinity-1 Fourier Transform Infrared Spectrophotometer and are reported in wavenumbers $\left(\mathrm{cm}^{-1}\right)$.

\section{Chromatography}

Chromatography was performed on silica gel (EMD, silica gel 60, particle size $0.040-0.063 \mathrm{~mm}$ ) using standard flash techniques or on $40 \mathrm{~g}$ HP Silica column (catalog 69-2203-347) using a Teledyne Isco Rf200 (detection at $210 \mathrm{~nm}$ ). Products were visualized by UV, iodine stain, ceric ammonium molybdate stain, or $\mathrm{KMnO}_{4}$ stain. 


\section{Coupling of (Hetero)Aromatic Acids with (Hetero)Aryl Halides}

\section{Optimization of the Coupling of 2-Methylbenzoic Acid with Aryl Halides}

Table S1. Effect of Aryl Electrophile and Additives on C-H Arylation Reaction.

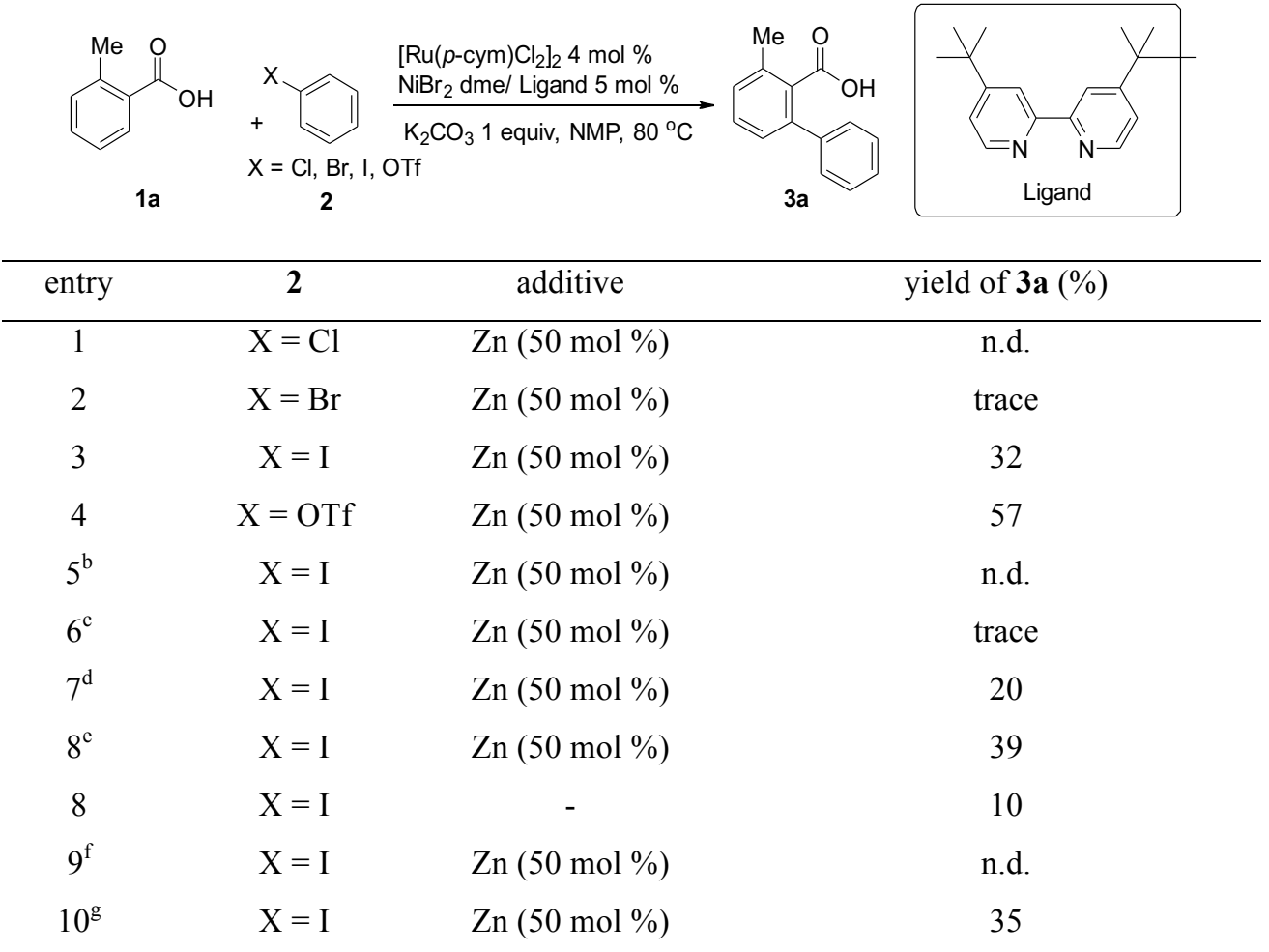

${ }^{\mathrm{a}}$ Reaction conditions: 1a $(0.25 \mathrm{mmol}), 2(0.375 \mathrm{mmol}),\left[\mathrm{Ru}(p \text {-cymene }) \mathrm{Cl}_{2}\right]_{2} 4 \mathrm{~mol} \%, \mathrm{NiBr}_{2} \mathrm{dme}(5 \mathrm{~mol} \%)$, dtbbpy (5 mol \%), $\mathrm{K}_{2} \mathrm{CO}_{3}$ (1 equiv), $80{ }^{\circ} \mathrm{C}, 24 \mathrm{~h}$. GC yield after esterification with $\mathrm{K}_{2} \mathrm{CO}_{3} / \mathrm{MeI}$, with $n$-dodecane as internal standard. ${ }^{\mathrm{b}} \mathrm{K}_{2} \mathrm{CO}_{3}$ (2 equiv) as base. ${ }^{\mathrm{c}} \mathrm{K}_{2} \mathrm{CO}_{3}$ (1 equiv), and $\mathrm{KOAc}$ ( 1 equiv) as base. ${ }^{\mathrm{d}} 60{ }^{\circ} \mathrm{C} .{ }^{\mathrm{e}} 100{ }^{\circ} \mathrm{C}$. ${ }^{\mathrm{f}}$ without Ligand. ${ }^{\mathrm{g}}$ without Ni-catalyst. 
Table S2. Ligand Effects in the Ru-Catalyzed C-H Arylation of $o$-Toluic Acid.

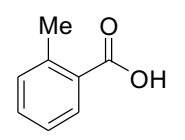

$1 \mathbf{a}$

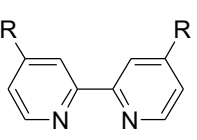

$\mathrm{L} 1, \mathrm{R}={ }^{t} \mathrm{Bu}$

$\mathrm{L} 2, \mathrm{R}=\mathrm{CF}_{3}$

$\mathrm{L} 3, \mathrm{R}=\mathrm{OMe}$

$\mathrm{PCy}_{3} \mathrm{~L} 10$
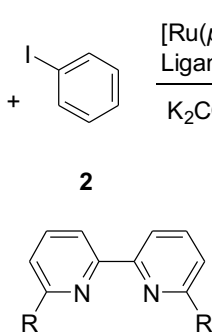

L4, $R=M e$

$\mathrm{L} 5, \mathrm{R}=\mathrm{Br}$

$\mathrm{PPh}_{3} \mathrm{~L} 11$

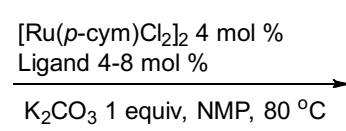

$\mathrm{K}_{2} \mathrm{CO}_{3} 1$ equiv, NMP, $80^{\circ} \mathrm{C}$

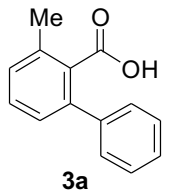

$3 a$<smiles>[R]c1ccnc(-c2cc([R])cc(-c3cc([R])ccn3)n2)c1</smiles>

$\mathrm{L} 6, \mathrm{R}=\mathrm{Me} \quad \mathrm{L} 8, \mathrm{R}=\mathrm{H}$

$\mathrm{L} 7, \mathrm{R}={ }^{t_{\mathrm{Bu}}} \quad \mathrm{L}, \mathrm{R}=\mathrm{NMe}_{2}$

$\mathrm{P}^{t} \mathrm{Bu}_{3} \mathrm{~L} 12 \quad \mathrm{P}(\text { o-toly })_{3} \mathrm{~L} 13$

$\mathrm{Ph}_{-\mathrm{P}^{-}} \mathrm{Ph} \mathrm{Ph}_{\mathrm{P}^{-}} \mathrm{Ph}$

$\mathrm{Ph} P \mathrm{Ph} \quad \mathrm{n}=2, \mathrm{~L} 14$ $\mathrm{Ph}^{-} \mathrm{P}_{Y Y_{n=2,4}}^{\mathrm{P}_{-}} \mathrm{Ph} \quad \mathrm{n}=4, \mathrm{~L} 15$
BINAP L17

DPPF L18

\begin{tabular}{|c|c|c|c|c|}
\hline Entry & $\begin{array}{l}\mathrm{Ru}] \\
(\%)\end{array}$ & $\begin{array}{c}\text { ligand } \\
(\%)\end{array}$ & solvent & $\begin{array}{l}\text { yield of } \\
\text { 3a (\%) }\end{array}$ \\
\hline 1 & 4 & $\mathrm{~L}_{1} 4 \mathrm{~mol} \%$ & NMP & 48 \\
\hline 2 & 4 & $\mathrm{~L}_{1} 8 \mathrm{~mol} \%$ & $"$ & 46 \\
\hline 3 & 4 & $\mathrm{~L}_{1} 4 \mathrm{~mol} \%$ & toluene & n.d. \\
\hline 4 & 4 & $\mathrm{~L}_{1} 4 \mathrm{~mol} \%$ & dioxane & trace \\
\hline 5 & 4 & $\mathrm{~L}_{1} 4 \mathrm{~mol} \%$ & DMAc & 41 \\
\hline 6 & 4 & $\mathrm{~L}_{1} 4 \mathrm{~mol} \%$ & DMF & 40 \\
\hline 7 & 4 & $\mathrm{~L}_{1} 4 \mathrm{~mol} \%$ & $\mathrm{H}_{2} \mathrm{O}$ & n.d. \\
\hline 8 & 4 & $\mathrm{~L}_{1} 4 \mathrm{~mol} \%$ & $i$-PrOH & n.d. \\
\hline 9 & 4 & $\mathrm{~L}_{2} 4 \mathrm{~mol} \%$ & NMP & n.d. \\
\hline 10 & 4 & $\mathrm{~L}_{3} 4 \mathrm{~mol} \%$ & $"$ & 58 \\
\hline 11 & 4 & $\mathrm{~L}_{4} 4 \mathrm{~mol} \%$ & $"$ & 67 \\
\hline $12^{\mathrm{b}}$ & 4 & $\mathrm{~L}_{4} 4 \mathrm{~mol} \%$ & $"$ & n.d. \\
\hline $13^{\mathrm{c}}$ & $"$ & $\mathrm{~L}_{4} 4 \mathrm{~mol} \%$ & $"$ & 54 \\
\hline $14^{\mathrm{d}}$ & $"$ & $\mathrm{~L}_{4} 4 \mathrm{~mol} \%$ & $"$ & 23 \\
\hline 15 & $"$ & $\mathrm{~L}_{5} 4 \mathrm{~mol} \%$ & $"$ & trace \\
\hline 16 & $"$ & $\mathrm{~L}_{6} 4 \mathrm{~mol} \%$ & $"$ & n.d. \\
\hline 17 & $"$ & $\mathrm{~L}_{7} 4 \mathrm{~mol} \%$ & $"$ & n.d. \\
\hline 18 & $"$ & $\mathrm{~L}_{8} 8 \mathrm{~mol} \%$ & $"$ & 12 \\
\hline 19 & $"$ & $\mathrm{~L}_{9} 8 \mathrm{~mol} \%$ & $"$ & 9 \\
\hline 20 & $"$ & $\mathrm{~L}_{10} 8 \mathrm{~mol} \%$ & $"$ & $91(94)$ \\
\hline 21 & 2 & $\mathrm{~L}_{10} 4 \mathrm{~mol} \%$ & & 83 \\
\hline 22 & 1 & $\mathrm{~L}_{10} 2 \mathrm{~mol} \%$ & & 54 \\
\hline 23 & $"$ & $\mathrm{~L}_{11} 8 \mathrm{~mol} \%$ & $"$ & 51 \\
\hline 24 & $"$ & $\mathrm{~L}_{12} 8 \mathrm{~mol} \%$ & $"$ & 28 \\
\hline 25 & 4 & $\mathrm{~L}_{13} 8 \mathrm{~mol} \%$ & $"$ & 9 \\
\hline 26 & 4 & $\mathrm{~L}_{14} 4 \mathrm{~mol} \%$ & $"$ & trace \\
\hline 27 & 4 & $\mathrm{~L}_{15} 4 \mathrm{~mol} \%$ & $"$ & 44 \\
\hline 28 & $"$ & $\mathrm{~L}_{16} 4 \mathrm{~mol} \%$ & $"$ & trace \\
\hline 29 & $"$ & $\mathrm{~L}_{17} 4 \mathrm{~mol} \%$ & $"$ & trace \\
\hline 30 & $"$ & $\mathrm{~L}_{18} 4 \mathrm{~mol} \%$ & $"$ & n.d. \\
\hline
\end{tabular}

${ }^{\mathrm{a}}$ Reaction conditions: 1a $(0.25 \mathrm{mmol}), 2(0.375 \mathrm{mmol})$, $\left[\mathrm{Ru}(p \text {-cymene }) \mathrm{Cl}_{2}\right]_{2} 1-4 \mathrm{~mol} \%$, ligand $(4-8 \mathrm{~mol} \%), \mathrm{K}_{2} \mathrm{CO}_{3}$ ( 1 equiv), $100{ }^{\circ} \mathrm{C}, 24 \mathrm{~h}$. GC yield after esterification with $\mathrm{K}_{2} \mathrm{CO}_{3} / \mathrm{MeI}$, with $n$-dodecane as internal standard. ${ }^{\mathrm{b}}$ $\mathrm{Ag}_{2} \mathrm{CO}_{3}$ (1 equiv) as base. ${ }^{\mathrm{c}} \mathrm{Cs}_{2} \mathrm{CO}_{3}$ (1 equiv) as base. ${ }^{\mathrm{d}} \mathrm{Na}_{2} \mathrm{CO}_{3}$ (1 equiv) as base. 
Table S3. C-H Arylation with Alternative Metals and PCy.

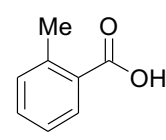

1a

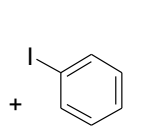

2

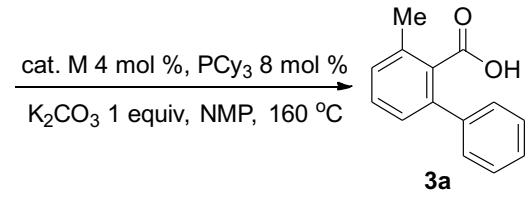

3a

\begin{tabular}{ccc}
\hline entry & cat. M & yield of $\mathbf{3 a}(\%)$ \\
\hline 1 & $\mathrm{CoBr}_{2}$ & n.d. \\
2 & $\mathrm{Cp}^{*} \mathrm{CoMe}_{2}$ & n.d. \\
3 & $\mathrm{CrCl}_{3}$ & n.d. \\
$4^{\mathrm{b}}$ & $\mathrm{RuCl}_{3} \cdot 3 \mathrm{H}_{2} \mathrm{O}$ & 68 \\
5 & $\mathrm{Cu}(\mathrm{OAc})_{2}$ & n.d. \\
6 & {$\left[\mathrm{IrCp} * \mathrm{Cl}_{2}\right]_{2}$} & 11 \\
$7^{\mathrm{b}}$ & {$\left[\mathrm{IrCp}^{*} \mathrm{Cl}_{2}\right]_{2}$} & 11 \\
$8^{\mathrm{c}}$ & {$\left[\mathrm{IrCp}^{*} \mathrm{Cl}_{2}\right]_{2}$} & 12
\end{tabular}

${ }^{\mathrm{a}}$ Reaction conditions: 1a $(0.25 \mathrm{mmol}), \mathbf{2 a}(0.375 \mathrm{mmol})$, cat. M (4 mol \%), $\mathrm{PCy}_{3}(8 \mathrm{~mol} \%), \mathrm{K}_{2} \mathrm{CO}_{3}$ (1 equiv), 160 ${ }^{\circ} \mathrm{C}$, $24 \mathrm{~h}$. GC yield after esterification with $\mathrm{K}_{2} \mathrm{CO}_{3} / \mathrm{MeI}$, with $n$-dodecane as internal standard. ${ }^{\mathrm{b}} 100{ }^{\circ} \mathrm{C} .{ }^{\mathrm{c}} 130{ }^{\circ} \mathrm{C}$.

\section{Additional Control and Mechanistic Experiments}

Amide vs carboxylate as directing group

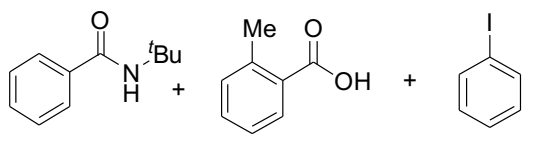

$0.25 \mathrm{mmol}$

$0.25 \mathrm{mmol}$

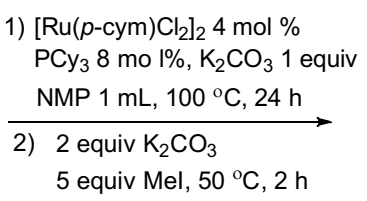
1.5 equiv

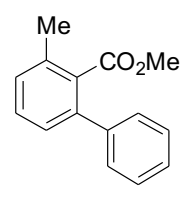

91\% NMR yield

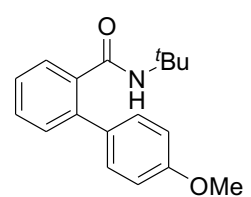

no detected

Ketone vs carboxylate as directing group<smiles>CC(=O)c1ccccc1</smiles>

$0.25 \mathrm{mmol}$<smiles>Cc1ccccc1C(=O)O</smiles>

$0.25 \mathrm{mmol}$<smiles>Ic1ccccc1</smiles>

1.5 equiv

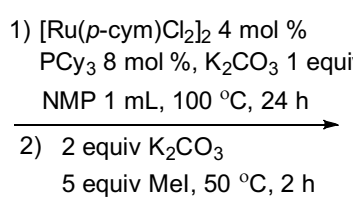

5 equiv Mel, $50{ }^{\circ} \mathrm{C}, 2 \mathrm{~h}$

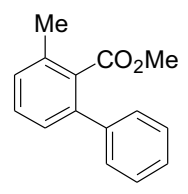

$96 \%$ NMR yield

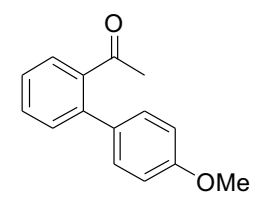

trace

Figure S1. Competition experiments between 2-methylbenzoic acid and $\mathrm{N}$-tert-

butylbenzamide and acetophenone. These results demonstrate that carboxylate over-rides both amide and ketone directing groups under these conditions.

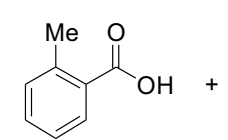

$0.25 \mathrm{mmol}$

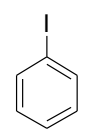

1.5 equiv

1) $\left[\mathrm{Ru}(p-\mathrm{cym}) \mathrm{Cl}_{2}\right]_{2} 4 \mathrm{~mol} \%$ $\mathrm{PCy}_{3} 8 \mathrm{~mol} \%, \mathrm{~K}_{2} \mathrm{CO}_{3} 1$ equiv NMP $1 \mathrm{~mL}, 100{ }^{\circ} \mathrm{C}, 24 \mathrm{~h}$

2) 2 equiv $\mathrm{K}_{2} \mathrm{CO}_{3}$ 5 equiv Mel, $50{ }^{\circ} \mathrm{C}, 2 \mathrm{~h}$

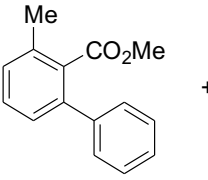

TEMPO 1 equiv as additive

BHT 1 equiv as additive $86 \%$ GC yield

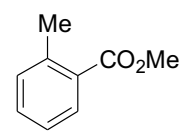

$77 \%$ GC yield

trace 
Figure S2. Radical trap experiments. When 1 equiv of TEMPO ( $\sim .25 \mathrm{M})$ was added to the reaction under standard conditions, nearly quantitative amounts of the starting material (77\% uncorrected GC yield vs dodecane as internal standard) was recovered. When the 1 equiv of BHT $(\sim 0.25 \mathrm{M})$ was added into the reaction, nearly all the carboxylic acid was transformed into the desired product ( $86 \%$ uncorrected GC yield vs dodecane as internal standard). These results are inconclusive for the involvement of radicals. While the TEMPO result is consistent with a radical intermediate, TEMPO can also react directly with transition metals. ${ }^{2}$ BHT did not affect the reaction, but this cannot rule out radicals that simply react faster in the desired pathway.

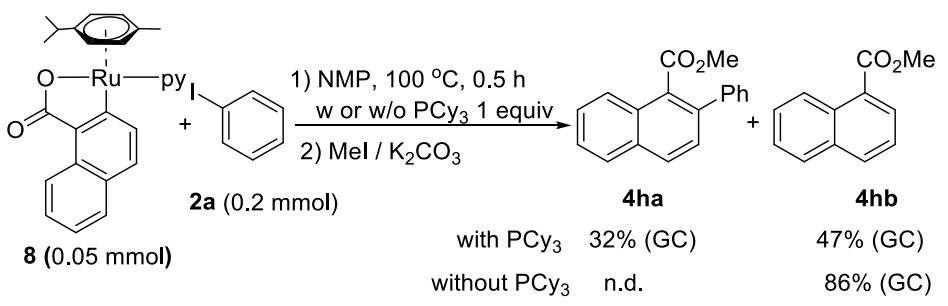

Figure S3. Stoichiometric Reaction. When ( $p$-cymeneene) $\mathrm{Ru}\left(\kappa^{2}-O, C\right.$-napthenoate $)(\mathrm{py}) \mathrm{Cl}(\mathbf{8})$ and $\mathrm{PhI}(\mathbf{2 a})$ were stirred in NMP $(0.2 \mathrm{~mL})$ at $100{ }^{\circ} \mathrm{C}$ for $30 \mathrm{mins}$, the desired product $4 \mathbf{h a}$ was formed only when $\mathrm{PCy}_{3}$ was present. (32\% yield vs. none detected). Without the phoshine ligand, only the methyl ester of starting material could be found.

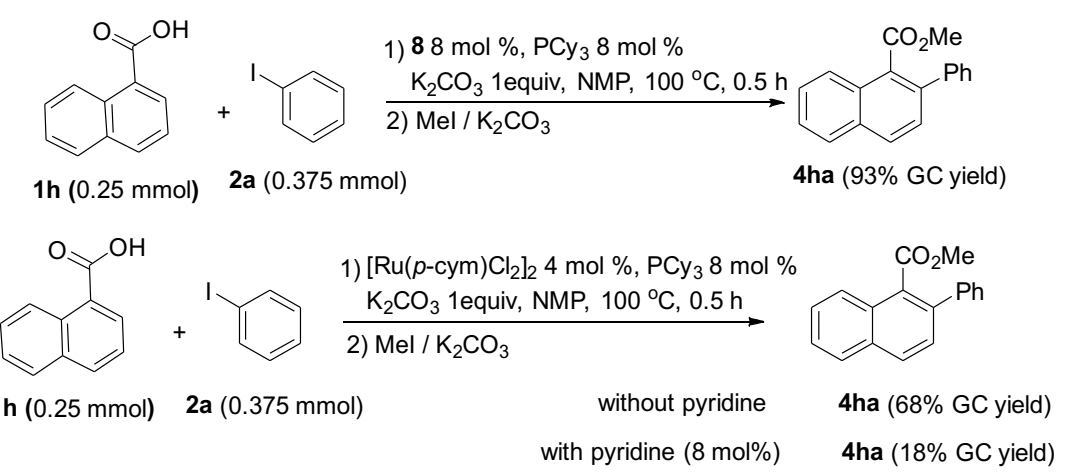

Figure S4. Kinetic Competency of Cyclometalated Complex 8. When complex 8 was used as a pre-catalyst, the standard reaction was complete in $0.5 \mathrm{~h}$. This is considerably faster than reactions catalyzed by $\left[(p \text {-cymene }) \mathrm{RuCl}_{2}\right]_{2}$ either with or without added pyridine. Note that the reaction with added pyridine was considerably slower to form product, consistent with the longer times required for heteroaryl halides.

\footnotetext{
${ }^{2}$ See, for example, the Cu/TEMPO oxidation of alcohols: Hoover, J. M.; Ryland, B. L.; Stahl, S. S. J. Am. Chem. Soc. 2013, $135,2357$.
} 


\section{General Procedure for the Coupling of 2-Methylbenzoic Acid and Aryl Halides.}
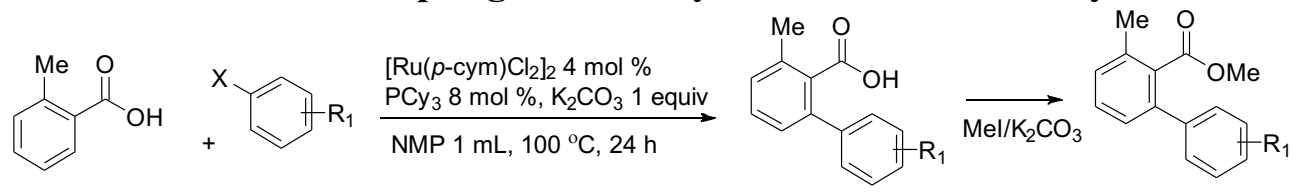

On the bench, an oven-dried 1-dram vial fitted with a Teflon-coated stir-bar was charged with $\left[\mathrm{Ru}(p \text {-cymene }) \mathrm{Cl}_{2}\right]_{2}(6.2 \mathrm{mg}, 0.01 \mathrm{mmol}, 4 \mathrm{~mol} \%), 2$-methylbenzoic acid (35 mg, $0.25 \mathrm{mmol}$, 1.0 equiv), and $\mathrm{K}_{2} \mathrm{CO}_{3}(34.5 \mathrm{mg}, 0.25 \mathrm{mmol}, 1.0$ equiv). The vial was moved into a nitrogen filled glove box and subeseqently charged with $\mathrm{PCy}_{3}(5.6 \mathrm{mg}, 0.02 \mathrm{mmol}, 8 \mathrm{~mol} \%$ ), dodecane (as an internal standard, $20.0 \mu \mathrm{L})$, aryl halide ( $0.375 \mathrm{mmol}, 1.50$ equiv), and NMP (1.00 mL). The vial was capped with a screw cap fitted with a PTFE-faced silicone septum, removed from the glove box, and heated in a reaction block set to $100^{\circ} \mathrm{C}$ on the benchtop with stirring at 1195 rpm for $24 \mathrm{~h}$.

After stirring for 24 hours, the reaction was removed from the plate and charged with $\mathrm{MeI}\left(0.1 \mathrm{~mL}, 1.6 \mathrm{mmol}, 6.4\right.$ equiv), another 2-3 equiv of $\mathrm{K}_{2} \mathrm{CO}_{3}(69-138 \mathrm{mg})$, and $\mathrm{MeCN}(0.5$ $\mathrm{mL}$ ). The resulting mixture was stirred for 2 hours at $60{ }^{\circ} \mathrm{C}$.

\section{GC Analysis.}

After the esterification reaction was complete, the reaction vial was vented to an oil bubbler before a $25 \mu \mathrm{L}$ aliquot of the reaction mixture was removed with a $50 \mu \mathrm{L}$ gas-tight syringe. The aliquot was diluted with diethyl ether $(1.5 \mathrm{~mL})$ and filtered through a short silica pad (about 1.5 $\mathrm{cm}$ ) in a pipette packed with glass wool. The filtrate was analyzed by gas chromatography and percent yield was calculated versus dodecane as the internal standard.

\section{Isolation and Purification}

When the reaction was judged complete, the reaction mixture was filtered through a short plug of silica gel $(1.5 \mathrm{~cm}$ wide $\times 2 \mathrm{~cm}$ high) to remove metal salts and the pad was washed with diethyl ether $(75 \mathrm{~mL})$. The filtrate was poured into a separatory funnel and partitioned with deionized water $(30 \mathrm{~mL})$. The aqueous layer was then extracted with diethyl ether $(3 \times 30 \mathrm{~mL})$. The combined organic layers were dried over $\mathrm{MgSO}_{4}$, filtered, and the filtrate was concentrated under reduced pressure. The resulting residue was purified by column chromatography on silica gel to afford the pure product. 


\section{Methyl 3-methylbiphenyl-2-carboxylate (3a) [CAS: 941320-77-0] ${ }^{3}$}<smiles>COC(=O)c1c(C)cccc1-c1ccccc1</smiles>

Compound 3a was prepared following the general procedure, starting from 2-methylbenzoic acid $(35 \mathrm{mg}, 0.25 \mathrm{mmol})$ and phenyl iodide $(76.5 \mathrm{mg}, 0.375 \mathrm{mmol})$. After purification by column chromatography (ethyl acetate / hexane $=10: 1$ ), 3a was obtained as colorless liquid (53.1 mg, 94\% isolated yield). ${ }^{1} \mathrm{H}$ NMR (400 MHz, $\left.\mathrm{CDCl}_{3}\right): \delta 7.36-7.32$ (m, 4H), $7.30-7.27$ $(\mathrm{m}, 2 \mathrm{H}), 7.19-7.16(\mathrm{~m}, 2 \mathrm{H}), 3.53(\mathrm{~s}, 3 \mathrm{H}), 2.36(\mathrm{~s}, 3 \mathrm{H}) ;{ }^{13} \mathrm{C} \mathrm{NMR}\left(100 \mathrm{MHz}, \mathrm{CDCl}_{3}\right): \delta 170.2$, $140.9,140.1,135.4,133.1,129.4,129.1,128.2,128.2,127.3,127.2,51.8,19.7$. MS (APCI+), m/z: $226.85\left[\mathrm{M}+\mathrm{H}^{+}\right]$. IR: $\tilde{v}=2947,2160,1975,1724,1435,1265,1123,1065,756,698$.

\section{Methyl 4'-chloro-3-methylbiphenyl-2-carboxylate (3b) [CAS: 1809272-61-4] $]^{3}$}<smiles>COC(=O)c1c(C)cccc1-c1ccc(Cl)cc1</smiles>

Compound $\mathbf{3 b}$ was prepared following the general procedure, starting from 2-methylbenzoic acid (35 mg, $0.25 \mathrm{mmol}$ ) and 1-chloro-4-iodobenzene ( $89.3 \mathrm{mg}, 0.375 \mathrm{mmol})$. After purification by column chromatography (ethyl acetate / hexane =10:1), 3a was obtained as colorless liquid (59.2 mg, 91\% isolated yield). ${ }^{1} \mathrm{H} \mathrm{NMR}\left(400 \mathrm{MHz}, \mathrm{CDCl}_{3}\right): \delta 7.36(\mathrm{t}, J=8.0 \mathrm{~Hz}, 3 \mathrm{H}), 7.30$ (d, $J=8.4 \mathrm{~Hz}, 2 \mathrm{H}), 7.23(\mathrm{~d}, J=7.6 \mathrm{~Hz}, 1 \mathrm{H}), 7.18(\mathrm{~d}, J=7.6 \mathrm{~Hz}, 1 \mathrm{H}), 3.62(\mathrm{~s}, 3 \mathrm{H}), 2.40(\mathrm{~s}, 3 \mathrm{H})$;

${ }^{13} \mathrm{C}$ NMR (100 MHz, $\left.\mathrm{CDCl}_{3}\right)$ : 170.0, 139.3, 138.8, 135.6, 133.5, 133.0, 129.5, 129.4, 128.4, 127.0, 51.9, 19.7. GCMS, m/z (\% relative intensity, ion): $262\left[\mathrm{M}+2^{+}\right](14), 260\left[\mathrm{M}^{+}\right](44), 231$ (21), 229 (67), 193 (36), 166 (64), 165 (100), 152 (9). IR: $\tilde{v}=2947,2160$, 1975, 1724, 1458, $1265,1123,1084,1065,787$.

\section{Methyl 4'-methoxy-3-methylbiphenyl-2-carboxylate (3c) [CAS: 1097018-19-3] ${ }^{3}$}<smiles>COC(=O)c1c(C)cccc1-c1ccc(OC)cc1</smiles>

Compound 3c was prepared following the general procedure, starting from 2-methoxybenzoic acid (35 mg, $0.25 \mathrm{mmol}$ ) and 1-iodo-4-methoxybenzene (87.8 $\mathrm{mg}, 0.375 \mathrm{mmol})$. After

\footnotetext{
${ }^{3}$ Huang, L.; Hackenberger, D.; Gooßen, L. J., Angew. Chem. Int. Ed. 2015, 54, 12607-12611.
} 
purification by column chromatography (ethyl acetate / hexane $=10: 1$ ), $\mathbf{3 c}$ was obtained as beige liquid (52.5 mg, 82\% isolated yield). ${ }^{1} \mathrm{H} \mathrm{NMR}$ (400 MHz, $\mathrm{CDCl}_{3}$ ): $\delta 7.33$ (dd, $J=16 \mathrm{~Hz}$, $8.0 \mathrm{~Hz}, 3 \mathrm{H}), 7.21-7.18(\mathrm{~m}, 2 \mathrm{H}), 6.93(\mathrm{~d}, J=12 \mathrm{~Hz}, 2 \mathrm{H}), 3.84(\mathrm{~s}, 3 \mathrm{H}), 3.63(\mathrm{~s}, 3 \mathrm{H}), 2.4(\mathrm{~s}, 3 \mathrm{H})$;

${ }^{13} \mathrm{C} \mathrm{NMR}\left(100 \mathrm{MHz}, \mathrm{CDCl}_{3}\right): \delta=170.5,159.0,139.6,135.2,133.2,133.1,129.3,128.7,127.1$, 113.7, 55.2, 51.8, 19.6. GCMS, m/z (\% relative intensity, ion): 256[ $\left.\mathrm{M}^{+}\right]$(100), 225 (84), 209 (27), 182 (47), 165 (44), 153 (66), 139 (27), 115 (17), 76 (8). IR: $\tilde{v}=2928,2160,1724,1609$, $1512,1246,1180,1088,787$.

\section{Dimethyl 3-methylbiphenyl-2,4'-dicarboxylate (3d) [CAS: 1809272-59-0] ${ }^{3}$}

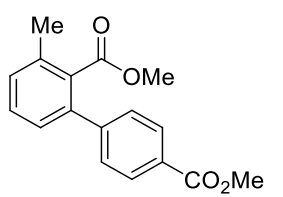

Compound 3d was prepared following procedure, starting from 2-methoxybenzoic acid (35 mg, $0.25 \mathrm{mmol})$, methyl-4-iodobenzoate $(98.3 \mathrm{mg}, 0.375 \mathrm{mmol})$. After purification by column chromatography (ethyl acetate / hexane $=10: 1)$, 3d was obtained as white solid $(62.5 \mathrm{mg}, 88 \%$ isolated yield). m.p. $=71-75{ }^{\circ} \mathrm{C} .{ }^{1} \mathrm{H}$ NMR $\left(400 \mathrm{MHz}, \mathrm{CDCl}_{3}\right): \delta 8.06(\mathrm{~d}, J=8.0 \mathrm{~Hz}, 2 \mathrm{H})$, $7.43(\mathrm{~d}, J=8.0 \mathrm{~Hz}, 2 \mathrm{H}), 7.37$ (t, $J=8.0 \mathrm{~Hz}, 1 \mathrm{H}), 7.23$ (dd, $J=15.2 \mathrm{~Hz}, 8.0 \mathrm{~Hz}, 2 \mathrm{H}), 3.93$ (s, 3H), $3.57(\mathrm{~s}, 3 \mathrm{H}), 2.40(\mathrm{~s}, 3 \mathrm{H}) ;{ }^{13} \mathrm{C} \mathrm{NMR}\left(100 \mathrm{MHz}, \mathrm{CDCl}_{3}\right): \delta 169.8,166.8,145.6,139.1$, $135.8,132.9,129.8,129.5,129.0,128.2,127.0,52.1,51.9,19.7$. GCMS, m/z (\% relative

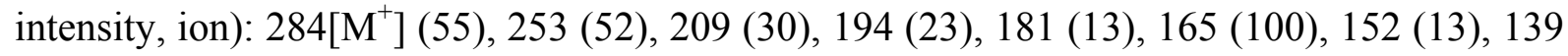
(12), 111 (9), 59 (15). IR: $\tilde{v}=2928,2160,1975,1709,1435,1261,1180,1115,1065,772$.

\section{Methyl 4'-acetyl-3-methylbiphenyl-2-carboxylate (3e) [CAS: 1809272-58-9] ${ }^{3}$}<smiles>COC(=O)c1c(OC)cccc1-c1ccc(C(C)=O)cc1</smiles>

Compound 3e was prepared following the general procedure, starting from 2-methoxybenzoic acid (35 mg, $0.25 \mathrm{mmol})$ and 1-(4-iodophenyl)ethanone (92.3 $\mathrm{mg}, 0.375 \mathrm{mmol})$. After purification by column chromatography (ethyl acetate / hexane $=10: 1$ ), 3e was obtained as brown oil (52.3 mg, 78\% isolated yield). m.p. $=124-127{ }^{\circ} \mathrm{C} .{ }^{1} \mathrm{H} \mathrm{NMR}\left(400 \mathrm{MHz}, \mathrm{CDCl}_{3}\right): \delta$ $7.98(\mathrm{~d}, J=8.0 \mathrm{~Hz}, 2 \mathrm{H}), 7.45(\mathrm{~d}, J=8.0 \mathrm{~Hz}, 2 \mathrm{H}), 7.37$ (t, $J=8.0 \mathrm{~Hz}, 1 \mathrm{H}), 7.25$ (d, $J=8.0 \mathrm{~Hz}$, 2H), $7.20(\mathrm{~d}, J=8.0 \mathrm{~Hz}, 1 \mathrm{H}), 3.59(\mathrm{~s}, 3 \mathrm{H}), 2.62(\mathrm{~s}, 3 \mathrm{H}), 2.40(\mathrm{~s}, 3 \mathrm{H}) ;{ }^{13} \mathrm{C} \mathrm{NMR}(100 \mathrm{MHz}$, $\left.\mathrm{CDCl}_{3}\right): \delta 197.7,169.8,145.7,139.0,135.9,135.8,132.9,129.8,129.5,128.4,128.3,127.0$, 
51.9, 26.6, 19.7. GCMS, m/z (\% relative intensity, ion): 268[ $\left.\mathrm{M}^{+}\right]$(46), 253 (100), 195 (38), 165 (62), 152 (16), 139 (11), 115 (8). IR: $\tilde{v}=2924,2160,1717,1678,1269,1180,1119,795$.

\section{Methyl 3-methyl-3'-(trifluoromethyl)biphenyl-2-carboxylate (3f)}<smiles>COC(=O)c1c(-c2cccc(C(F)(F)F)c2)cccc1[N+](=O)[O-]</smiles>

Compound $3 \mathbf{f}$ was prepared following the general procedure, starting from 2-methoxybenzoic acid (35 mg, $0.25 \mathrm{mmol}$ ) and 1-iodo-3-(trifluoromethyl)benzene (102 mg, $0.375 \mathrm{mmol}$ ). After purification by column chromatography (ethyl acetate / hexane $=10: 1$ ), 3f was obtained as colorless liquid (61.7 mg, 84\% isolated). ${ }^{1} \mathrm{H}$ NMR (400 MHz, $\left.\mathrm{CDCl}_{3}\right): \delta 7.65(\mathrm{~s}, 1 \mathrm{H}), 7.62(\mathrm{~d}$, $J=7.6 \mathrm{~Hz}, 1 \mathrm{H}), 7.57-7.50(\mathrm{~m}, 2 \mathrm{H}), 7.39$ (t, $J=7.6 \mathrm{~Hz}, 1 \mathrm{H}), 7.27$ (d, $J=7.6 \mathrm{~Hz}, 1 \mathrm{H}), 7.22$ (d, $J=7.6 \mathrm{~Hz}, 1 \mathrm{H}), 3.62(\mathrm{~s}, 3 \mathrm{H}), 2.42(\mathrm{~s}, 3 \mathrm{H}) ;{ }^{13} \mathrm{C} \mathrm{NMR}\left(100 \mathrm{MHz}, \mathrm{CDCl}_{3}\right): \delta 169.8,141.6,138.5$, 135.8, 133.2, 131.6, 130.7 (q, $J=25.6 \mathrm{~Hz}$ ), 129.8, 129.6, 128.8, 127.1, 125.0 (unresolved m), 124.1 (unresolved m), 124.0 (q, $J=216.4 \mathrm{~Hz}), 51.8,19.7 .{ }^{19} \mathrm{~F} \mathrm{NMR}\left(376 \mathrm{MHz}, \mathrm{CDCl}_{3}\right): \delta 0.22$ (s, 3F). GCMS, m/z (\% relative intensity, ion): 294[M $\left.\mathrm{M}^{+}\right]$(52), 275 (8), 263 (100), 215 (27), 165 (87), 139 (4), 107 (5). IR: $\tilde{v}=2928,2160,1728,1435$, 1335, 1265, 1165, 1119, 1069, 783, 702.

\section{3',5'-Dimethoxy-3-methylbiphenyl-2-carboxylic acid (3g)}

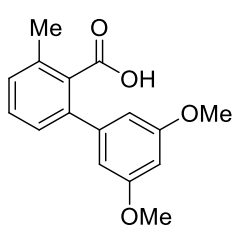

Compound $\mathbf{3 g}$ was prepared following the general procedure, starting from 2-methoxybenzoic acid (35 mg, $0.25 \mathrm{mmol}$ ) and 1-bromo-3,5-dimethoxybenzene (81 $\mathrm{mg}, 0.375 \mathrm{mmol})$. After purification by column chromatography (ethyl acetate / hexane $=5: 1$ ), $\mathbf{3 g}$ was obtained as light yellow solid (44.3 mg, $62 \%$ isolated yield). m.p. $=70-74{ }^{\circ} \mathrm{C} .{ }^{1} \mathrm{H} \mathrm{NMR}\left(400 \mathrm{MHz}, \mathrm{CDCl}_{3}\right): \delta$ 7.35 (t, $J=7.6 \mathrm{~Hz}, 1 \mathrm{H}), 7.23(\mathrm{t}, J=7.2 \mathrm{~Hz}, 2 \mathrm{H}), 6.53(\mathrm{~d}, J=2.4 \mathrm{~Hz}, 2 \mathrm{H}), 6.45(\mathrm{~m}, J=2.0 \mathrm{~Hz}$, $1 \mathrm{H}), 3.80(\mathrm{~s}, 6 \mathrm{H}), 3.65(\mathrm{~s}, 3 \mathrm{H}), 2.39(\mathrm{~s}, 3 \mathrm{H}) ;{ }^{13} \mathrm{C} \mathrm{NMR}\left(100 \mathrm{MHz}, \mathrm{CDCl}_{3}\right): \delta 170.3,160.6$, 142.9, 140.0, 135.4, 133.1, 129.4, 129.3, 127.0, 106.3, 99.9, 55.4, 52.0, 19.6. MS (APCI+), m/z: $286.85\left[\mathrm{M}+\mathrm{H}^{+}\right] . \mathrm{IR}: \tilde{v}=2928,2160,2018,1975,1721,1585,1416,1273,1204,1153,1065$, 787. 


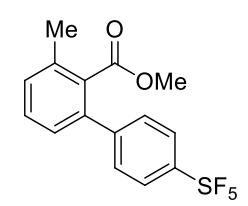

Compound $3 \mathbf{h}$ was prepared following the general procedure, starting from 2-methoxybenzoic acid (35 mg, $0.25 \mathrm{mmol}$ ) and 1-bromo-4-pentafluorosulfanylbenzene (105.8 mg, $0.375 \mathrm{mmol}$ ). After purification by column chromatography (ethyl acetate / hexane $=10: 1$ ), $3 \mathbf{h}$ was obtained as colorless liquid (62.1 mg, 71\% isolated yield). ${ }^{1} \mathrm{H}$ NMR (400 $\left.\mathrm{MHz}, \mathrm{CDCl}_{3}\right): \delta 7.78(\mathrm{~d}, J=$ $8.4 \mathrm{~Hz}, 2 \mathrm{H}), 7.45$ (d, $J=8.4 \mathrm{~Hz}, 2 \mathrm{H}), 7.39$ (t, $J=8.0 \mathrm{~Hz}, 1 \mathrm{H}), 7.28$ (d, $J=7.6 \mathrm{~Hz}, 1 \mathrm{H}), 7.19$ $(\mathrm{d}, J=7.6 \mathrm{~Hz}, 1 \mathrm{H}), 3.62(\mathrm{~s}, 3 \mathrm{H}), 2.42(\mathrm{~s}, 3 \mathrm{H}) ;{ }^{13} \mathrm{C} \mathrm{NMR}\left(100 \mathrm{MHz}, \mathrm{CDCl}_{3}\right): \delta 169.7,152.8$ (unresolved m), 144.4, 138.0, 136.0, 133.0, 130.1. 129.7, 128.5, 127.1, 125.9 (quint, $J=4 \mathrm{~Hz}$ ), 52.0, 19.7. ${ }^{19} \mathrm{~F}$ NMR $\left(376 \mathrm{MHz}, \mathrm{CDCl}_{3}\right.$ ): $\delta 147.3$ (quint, $\left.J=151.2 \mathrm{~Hz}, 1 \mathrm{~F}\right), 125.9(\mathrm{~d}, J=150.0$

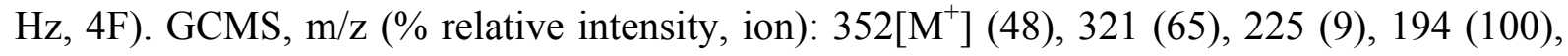
165 (67), 152 (9), 139 (8), 115 (6). IR: $\tilde{v}=2927,2160,1728,1439,1269,1123,1069,821,787$.

\section{Methyl 4'-fluoro-3-methylbiphenyl-2-carboxylate (3i) [CAS: 1809272-60-3] ${ }^{3}$}<smiles>COC(=O)c1c(-c2ccc(F)cc2)cccc1[N+](=O)[O-]</smiles>

Compound 3i was prepared following the general procedure, starting from 2-methoxybenzoic acid (35 mg, $0.25 \mathrm{mmol}$ ) and 1-bromo-4-fluorobenzene (65.3 $\mathrm{mg}, 0.375 \mathrm{mmol})$. After purification by column chromatography (ethyl acetate / hexane $=10: 1), \mathbf{3 i}$ was obtained as colorless liquid (45.8 mg, 75\% isolated yield). m.p. $=60-64{ }^{\circ} \mathrm{C} .{ }^{1} \mathrm{H} \mathrm{NMR}\left(400 \mathrm{MHz}, \mathrm{CDCl}_{3}\right)$ : $\delta 7.37-7.31(\mathrm{~m}, 3 \mathrm{H}), 7.22(\mathrm{~d}, J=7.2 \mathrm{~Hz}, 1 \mathrm{H}), 7.18(\mathrm{~d}, J=7.6 \mathrm{~Hz}, 1 \mathrm{H}), 7.10-7.06(\mathrm{~m}, 2 \mathrm{H})$, $3.61(\mathrm{~s}, 3 \mathrm{H}), 2.40(\mathrm{~s}, 3 \mathrm{H}) ;{ }^{13} \mathrm{C} \mathrm{NMR}\left(100 \mathrm{MHz}, \mathrm{CDCl}_{3}\right): \delta 170.2,162.3(\mathrm{~d}, J=244.9 \mathrm{~Hz}), 139.0$, $136.9(\mathrm{~d}, J=3.2 \mathrm{~Hz}), 135.5,133.2,129.8$ (d, $J=32.4 \mathrm{~Hz}), 129.3$ (d, $J=82 \mathrm{~Hz}), 127.2,115.3$, 115.1, 51.9, 19.7. ${ }^{19} \mathrm{~F}$ NMR (376 MHz, $\left.\mathrm{CDCl}_{3}\right): \delta-52.4(\mathrm{~m}, 1 \mathrm{~F})$. GCMS, m/z (\% relative intensity, ion): 244 (100) $\left[\mathrm{M}^{+}\right], 214$ (13), 213 (88), 212 (26), 183 (11), 165 (11). IR: $\tilde{v}=2924$, $2160,2025,1735,1508,1261,1119,1088,1065,841,767$.

\section{3',5'-Di-tert-butyl-4'-hydroxy-3-methylbiphenyl-2-carboxylic acid (3j)}

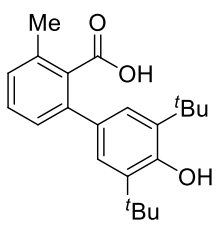


Compound 3j was prepared following the general procedure, starting from 2-methoxybenzoic acid (35 mg, $0.25 \mathrm{mmol}$ ) and 2,6-di-tert-butyl-4-bromophenol (106.5 mg, $0.375 \mathrm{mmol})$. After the reaction was complete, the reaction mixture was allowed to cool to rt. The reaction mixture was diluted with ethyl acetate $(20 \mathrm{~mL})$ and extracted with aq. $\mathrm{NaOH}$ solution $(3 \times 20 \mathrm{~mL})$. The aq. basic phase was acidified with $\mathrm{HCl}(\mathrm{pH} 1-2)$ and afterwards extracted with ethyl acetate (3 $\times 20 \mathrm{~mL}$ ). The combined organic layers were dried over $\mathrm{MgSO}_{4}$, filtered, and the volatiles were removed under reduced pressure. The residue was purified by column chromatography $\left(\mathrm{SiO}_{2}\right.$, ethyl acetate/hexane $=10: 1,1 \%$ formic acid $)$ yielding $\mathbf{3} \mathbf{j}$ as a beige solid $(57.8 \mathrm{mg}, 68 \%$ isolated yield). m.p. $=203-210{ }^{\circ} \mathrm{C} .{ }^{1} \mathrm{H}$ NMR $\left(400 \mathrm{MHz}, \mathrm{CDCl}_{3}\right): \delta 7.36(\mathrm{t}, J=8.0 \mathrm{~Hz}, 1 \mathrm{H}), 7.28-7.26$ $(\mathrm{m}, 3 \mathrm{H}), 7.19(\mathrm{~d}, J=8.0 \mathrm{~Hz}, 1 \mathrm{H}), 5.24(\mathrm{~s}, 1 \mathrm{H}), 2.40(\mathrm{~s}, 3 \mathrm{H}), 1.45(\mathrm{~s}, 18 \mathrm{H}) ;{ }^{13} \mathrm{C} \mathrm{NMR}(100 \mathrm{MHz}$, $\left.\mathrm{CDCl}_{3}\right): \delta 176.4,153.5,140.8,135.8,135.1,132.3,131.5,129.6,128.4,127.4,125.2,34.4$, 30.3, 19.9. MS (APCI+), m/z: $340.95\left[\mathrm{M}+\mathrm{H}^{+}\right]$. IR: $\tilde{v}=2924,2523,2160,1686,1431,1300$, $1231,887$.

\section{4'-Hydroxy-3-methylbiphenyl-2-carboxylic acid (3k) [CAS: 1261914-93-5] ${ }^{4}$}<smiles>Cc1cccc(-c2ccc(O)cc2)c1C(=O)O</smiles>

Compound 3k was prepared following the general procedure, starting from 2-methoxybenzoic acid (35 mg, $0.25 \mathrm{mmol})$ and 4-bromophenol (64.5 $\mathrm{mg}, 0.375 \mathrm{mmol})$. After the reaction was complete, the reaction mixture was allowed to cool to rt. The reaction mixture was diluted with ethyl acetate $(20 \mathrm{~mL})$ and extracted with aq. $\mathrm{NaOH}$ solution $(3 \times 20 \mathrm{~mL})$. The aq. basic phase was acidified with $\mathrm{HCl}(\mathrm{pH} 1-2)$ and afterwards extracted with ethyl acetate $(3 \times 20 \mathrm{~mL})$. The combined organic layers were dried over $\mathrm{MgSO}_{4}$, filtered, and the volatiles were removed under reduced pressure. The residue was purified by column chromatography $\left(\mathrm{SiO}_{2}\right.$, ethyl acetate/hexane $=10: 1,1 \%$ formic acid) yielding $3 \mathbf{k}$ as beige solid ( $30.2 \mathrm{mg}, 53 \%$ isolated yield). m.p. $=192-194{ }^{\circ} \mathrm{C} .{ }^{1} \mathrm{H}$ NMR $\left(400 \mathrm{MHz}, \mathrm{CD}_{3} \mathrm{CN}\right): \delta 7.40(\mathrm{t}, J=8.0 \mathrm{~Hz}, 1 \mathrm{H}), 7.30-7.28(\mathrm{~m}$, $3 \mathrm{H}), 7.22(\mathrm{~d}, J=8.0 \mathrm{~Hz}, 1 \mathrm{H}), 6.89(\mathrm{~d}, J=8.0 \mathrm{~Hz}, 2 \mathrm{H}), 2.42(\mathrm{~s}, 3 \mathrm{H}) ;{ }^{13} \mathrm{C} \mathrm{NMR}(100 \mathrm{MHz}$, $\left.\mathrm{CD}_{3} \mathrm{CN}\right): \delta 170.4,156.6,138.9,134.6,133.6,132.2,129.6,129.2,128.5,127.1,115.1,18.8$. MS (APCI+), m/z: $228.85\left[\mathrm{M}+\mathrm{H}^{+}\right] . \mathrm{IR}: \tilde{v}=2928,2160,1662,1516,1265,1211,1169,829$, 775.

\footnotetext{
${ }^{4}$ Wu, Z.; Luo, F.; Chen, S.; Li, Z.; Xiang, H.; Zhou, X., Chem. Commun. 2013, 49, 7653-7655.
} 
Methyl 3'-bromo-4'-methoxy-3-methylbiphenyl-2-carboxylate (3I)<smiles>COc1cc(-c2cccc(Br)c2OC)ccc1O[AlH2]</smiles>

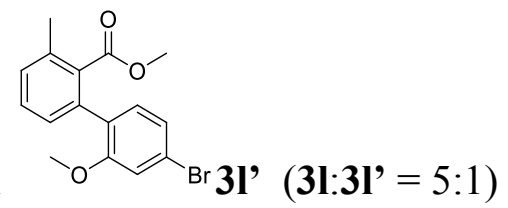

Compound $3 \mathbf{l}$ was prepared following the general procedure, starting from 2-methoxybenzoic acid (35 mg, $0.25 \mathrm{mmol}$ ) and 2,4-dibromo-1-methoxybenzene (99 mg, $0.375 \mathrm{mmol}$ ). After purification by column chromatography (ethyl acetate / hexane $=10: 1$ ), 3ol was obtained as yellow solid (79.3 mg, 95\% isolated yield). m.p. $=84-92{ }^{\circ} \mathrm{C} .{ }^{1} \mathrm{H} \mathrm{NMR}\left(400 \mathrm{MHz}, \mathrm{CDCl}_{3}\right): \delta$ $7.58(\mathrm{~d}, J=2.4 \mathrm{~Hz}, 1 \mathrm{H}), 7.34(\mathrm{t}, J=7.6 \mathrm{~Hz}, 1 \mathrm{H}), 7.29-7.27(\mathrm{~m}, 1 \mathrm{H}), 7.20(\mathrm{~d}, J=7.6 \mathrm{~Hz}, 1 \mathrm{H})$, $7.17(\mathrm{~d}, J=8.0 \mathrm{~Hz}, 1 \mathrm{H}), 6.91$ (d, $J=8.8 \mathrm{~Hz}, 1 \mathrm{H}), 3.92$ (s, 3H), 3.67 (s, 3H), 3.38 (s, 3H); ${ }^{13} \mathrm{C} \mathrm{NMR}\left(100 \mathrm{MHz}, \mathrm{CDCl}_{3}\right): \delta 170.2,155.2,138.1,135.5,134.6,133.1,129.4,129.2,128.3$, 127.1, 111.5, 56.2, 51.9, 19.7. MS (APCI+), m/z: $334.75\left[\mathrm{M}+\mathrm{H}^{+}\right]$. IR: $\tilde{v}=2947,1724,1501$, $1439,1265,1119,1096,1053,802$.

\section{Methyl 3'-bromo-3,4'-dimethylbiphenyl-2-carboxylate (3m)}<smiles>Cc1ccc(-c2cccc(C)c2C(=O)O)c(Br)c1</smiles>

Compound 3m was prepared following the general procedure, starting from 2-methoxybenzoic acid (35 mg, $0.25 \mathrm{mmol}$ ) and 2-bromo-4-iodo-1-methylbenzene (111 mg, $0.375 \mathrm{mmol})$. After purification by column chromatography (ethyl acetate / hexane =10:1), 3m was obtained as light yellow liquid (73.9 mg, 93\% isolated yield). ${ }^{1} \mathrm{H}$ NMR (400 MHz, $\left.\mathrm{CDCl}_{3}\right): \delta 7.58(\mathrm{~s}, 1 \mathrm{H})$, $7.35(\mathrm{t}, J=8.0 \mathrm{~Hz}, 1 \mathrm{H}), 7.26-7.18(\mathrm{~m}, 4 \mathrm{H}), 3.67(\mathrm{~s}, 3 \mathrm{H}), 2.43(\mathrm{~s}, 2 \mathrm{H}), 2.40(\mathrm{~s}, 3 \mathrm{H}) ;{ }^{13} \mathrm{C} \mathrm{NMR}$ $\left(100 \mathrm{MHz}, \mathrm{CDCl}_{3}\right): \delta 170.1,140.1,138.3,136.9,135.5,133.1,131.9,130.5,129.5,129.4$, 127.1, 124.8, 51.9, 22.6, 19.7. MS (APCI+), m/z: $318.70\left[\mathrm{M}+\mathrm{H}^{+}\right] . \mathrm{IR}: \tilde{v}=2947,1724,1497$, $1435,1265,1238,1119,1092,1065,787$.

Methyl 4'-bromo-3-methylbiphenyl-2-carboxylate (3n) [CAS: 809272-62-5] ${ }^{3}$<smiles>COC(=O)c1c(-c2ccc(Br)cc2)cccc1[N+](=O)[O-]</smiles> 
Compound 3n was prepared following the general procedure, starting from 2-methoxybenzoic acid (35 mg, $0.25 \mathrm{mmol})$ and 1-bromo-4-iodobenzene (211.5 mg, $0.75 \mathrm{mmol})$. After purification by column chromatography (ethyl acetate / hexane $=10: 1$ ), $\mathbf{3 n}$ and $\mathbf{3 n}$ ' were obtained as light yellow solid with about 6:1 ratio (59.3 mg, 78\% isolated yield). m.p. $=54-55$ ${ }^{\circ} \mathrm{C} .{ }^{1} \mathrm{H}$ NMR (400 MHz, $\left.\mathrm{CDCl}_{3}\right): \delta$ 7.43-7.40 (m, 2H), 7.25 (t, $\left.J=7.6 \mathrm{~Hz}, 1 \mathrm{H}\right), 7.15-7.12(\mathrm{~m}$, $3 \mathrm{H}), 7.07(\mathrm{~d}, J=7.6 \mathrm{~Hz}, 1 \mathrm{H}), 3.52(\mathrm{~s}, 3 \mathrm{H}), 2.30(\mathrm{~s}, 3 \mathrm{H}) ;{ }^{13} \mathrm{C} \mathrm{NMR}\left(100 \mathrm{MHz}, \mathrm{CDCl}_{3}\right): \delta 170.0$, $139.8,138.8,135.6,132.9,131.4,129.8,129.5,129.4,127.0,121.7,51.9,19.6$. GCMS, m/z (\% relative intensity, ion): $306\left[\mathrm{M}+2^{+}\right](30), 304\left[\mathrm{M}^{+}\right](32), 275$ (23), 273 (24), 194 (91), 193 (40), 165 (100). IR: $\tilde{v}=2947,1724,1589,1458,1435,1265,1238,1119,1069,787$.

\section{Methyl 3'-bromo-3-methylbiphenyl-2-carboxylate (3o) [CAS: 1809272-66-9] ${ }^{3}$}

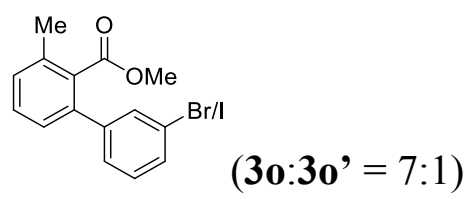

Compound 3o was prepared following the general procedure, starting from 2-methoxybenzoic acid (35 mg, $0.25 \mathrm{mmol}$ ) and 1-bromo-3-iodobenzene (211.5 mg, $0.75 \mathrm{mmol})$. After purification by column chromatography (ethyl acetate / hexane $=10: 1$ ), 3o and 3o' were obtained as brown oil with about 7:1 ratio (62.3 mg, 82\% isolated yield). ${ }^{1} \mathrm{H}$ NMR (400 MHz, $\left.\mathrm{CDCl}_{3}\right): \delta 7.52(\mathrm{t}, J=1.6 \mathrm{~Hz}, 1 \mathrm{H}), 7.45(\mathrm{dt}, J=7.6 \mathrm{~Hz}, 1.8 \mathrm{~Hz}, 1 \mathrm{H}), 7.35(\mathrm{t}, J=7.8 \mathrm{~Hz}, 1 \mathrm{H})$, 7.29-7.26 (m, 1H), 7.25-7.22 (m, 2H), $7.18(\mathrm{~d}, J=7.6 \mathrm{~Hz}, 1 \mathrm{H}), 3.62(\mathrm{~s}, 3 \mathrm{H}), 2.39(\mathrm{~s}, 3 \mathrm{H}) ;{ }^{13} \mathrm{C}$ NMR (100 MHz, $\left.\mathrm{CDCl}_{3}\right): \delta 169.9,142.9,138.5,135.7,133.1,131.3,130.4,129.8,129.7$, $129.5,127.1,126.9,122.3,51.9,19.7 . \mathrm{GCMS}, \mathrm{m} / \mathrm{z}$ (\% relative intensity, ion): $306\left[\mathrm{M}+2^{+}\right](25)$, $304\left[\mathrm{M}^{+}\right]$(27), 275 (22), 273 (22), 194 (84), 165 (100), 139 (12). IR: $\tilde{v}=2947,1724,1589$, $1555,1454,1265,1238,1123,1099,1065,775$.

\section{Methyl 2-methyl-6-(thiophen-3-yl)benzoate (3p)}<smiles>COc1c(C)cccc1-c1ccsc1</smiles>

Compound 3p was prepared following the general procedure, starting from 2-methoxybenzoic acid (35 $\mathrm{mg}, 0.25 \mathrm{mmol}$ ) and 3-bromothiophene $(60.8 \mathrm{mg}, 0.375 \mathrm{mmol})$. After purification by column chromatography (ethyl acetate / hexane $=10: 1$ ), 3p was obtained as light yellow solid 
(46.4 mg, 80\% isolated yield). m.p. $=65-66^{\circ} \mathrm{C} .{ }^{1} \mathrm{H}$ NMR $\left(400 \mathrm{MHz}, \mathrm{CDCl}_{3}\right): \delta 7.36-7.36$ (m, 2H), $7.31-7.28(\mathrm{~m}, 2 \mathrm{H}), 7.19(\mathrm{~d}, J=8.0 \mathrm{~Hz}, 1 \mathrm{H}), 7.16(\mathrm{dd}, J=8.0 \mathrm{~Hz}, 4.0 \mathrm{~Hz}, 1 \mathrm{H}), 3.70$ (s, 3H), $2.38(\mathrm{~s}, 3 \mathrm{H}) ;{ }^{13} \mathrm{C} \mathrm{NMR}\left(100 \mathrm{MHz}, \mathrm{CDCl}_{3}\right): \delta 170.5,140.9,135.2,134.3,133.0,129.3$, 129.0, 127.9, 126.8, 125.6, 122.3, 52.0, 19.5. GCMS, m/z (\% relative intensity, ion): $232\left[\mathrm{M}^{+}\right]$ (56), 200 (100), 171 (78), 158 (20), 129 (31), 115 (19), 102 (6). IR: $\tilde{v}=2924,1728,1258$, $1119,1065,772$.

\section{Methyl 2-methyl-6-(1-methyl-1H-pyrazol-4-yl)benzoate (3q)}<smiles>COC(=O)c1c(-c2cnn(C)c2)cccc1[N+](=O)[O-]</smiles>

Compound 3q was prepared following the general procedure, starting from 2-methoxybenzoic acid (35 mg, $0.25 \mathrm{mmol})$ and 3-iodo-1-methyl-1H-pyrazole (78 mg, $0.375 \mathrm{mmol})$. After purification by column chromatography (ethyl acetate / hexane $=5: 1$ ), $\mathbf{3 q}$ was obtained as colorless liquid $\left(47.2 \mathrm{mg}, 82 \%\right.$ isolated yield). ${ }^{1} \mathrm{H}$ NMR $\left(400 \mathrm{MHz}, \mathrm{CDCl}_{3}\right): \delta 7.55(\mathrm{~s}, 1 \mathrm{H})$, $7.28(\mathrm{t}, J=8.0 \mathrm{~Hz}, 1 \mathrm{H}), 7.20(\mathrm{~d}, J=8.0 \mathrm{~Hz}, 1 \mathrm{H}), 7.12(\mathrm{~d}, J=8.0 \mathrm{~Hz}, 1 \mathrm{H}), 3.91(\mathrm{~s}, 3 \mathrm{H}), 3.79$ (s, $3 \mathrm{H}), 2.33(\mathrm{~s}, 3 \mathrm{H}) ;{ }^{13} \mathrm{C} \mathrm{NMR}\left(100 \mathrm{MHz}, \mathrm{CDCl}_{3}\right): \delta 170.8,138.0,134.9,132.6,129.9,129.4$, 128.4, 128.3, 126.3, 121.1, 52.1, 39.0, 19.4. GCMS, m/z (\% relative intensity, ion): $230\left[\mathrm{M}^{+}\right]$ (100), 198 (83), 184 (17), 169 (16), 155 (25), 130 (15), 115 (21), 103 (23), 77 (14). IR: $\tilde{v}=$ 2947, 1724, 1593, 1439, 1265, 1099, 1069, 787.

\section{Methyl 2-methyl-6-(1-methyl-1H-indol-5-yl)benzoate (3r)}

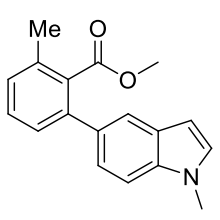

Compound 3r was prepared following the general procedure, starting from 2-methoxybenzoic acid (35 mg, $0.25 \mathrm{mmol}$ ) and 5-iodo-1-methyl-1H-indole (96.4 mg, $0.375 \mathrm{mmol})$. After purification by column chromatography (ethyl acetate / hexane $=10: 1$ ), 3r was obtained as yellow solid (65.5 mg, 94\% isolated yield). m.p. $=83-84{ }^{\circ} \mathrm{C} .{ }^{1} \mathrm{H} \mathrm{NMR}\left(400 \mathrm{MHz}, \mathrm{CDCl}_{3}\right): \delta$ $7.66(\mathrm{~s}, 1 \mathrm{H}), 7.39-7.34(\mathrm{~m}, 2 \mathrm{H}), 7.32$ (d, $J=8.0 \mathrm{~Hz}, 1 \mathrm{H}), 7.28(\mathrm{dd}, J=8.4 \mathrm{~Hz}, 1.6 \mathrm{~Hz}, 1 \mathrm{H})$, $7.21(\mathrm{~d}, J=7.2 \mathrm{~Hz}, 1 \mathrm{H}), 7.09$ (d, $J=2.8 \mathrm{~Hz}, 1 \mathrm{H}), 6.52(\mathrm{~d}, J=3.2 \mathrm{~Hz}, 1 \mathrm{H}), 3.82(\mathrm{~s}, 3 \mathrm{H}), 3.60$ (s, 3H), $2.44(\mathrm{~s}, 3 \mathrm{H}) ;{ }^{13} \mathrm{C} \mathrm{NMR}\left(100 \mathrm{MHz}, \mathrm{CDCl}_{3}\right): \delta 170.8,141.2,136.1,135.1,133.5,132.1$, 
$129.3,129.2,128.5,128.3,127.7,122.2,120.5,109.0,101.2,51.8,32.8,19.7$. GCMS, m/z (\% relative intensity, ion): $279\left[\mathrm{M}^{+}\right]$(100), 247 (73), 233 (16), 218 (11), 204 (15), 178 (6), 151 (5), 139 (6), 124 (8), 102 (15), 97 (26). IR: $\tilde{v}=2978,1728,1265,1242,787$.

\section{Methyl 2-(6-chloro-5-methylpyridin-3-yl)-6-methylbenzoate (3s)}

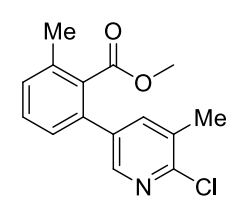

Compound 3s was prepared following the general procedure, starting from 2-methoxybenzoic acid (35 mg, $0.25 \mathrm{mmol}$ ) and 5-bromo-2-chloro-3-methylpyridine (76.9 mg, $0.375 \mathrm{mmol}$ ). After purification by column chromatography (ethyl acetate / hexane $=5: 1$ ), 3s was obtained as yellow liquid (28.9 mg, 42\% isolated yield). ${ }^{1} \mathrm{H} \mathrm{NMR}\left(400 \mathrm{MHz}, \mathrm{CDCl}_{3}\right): \delta 8.22$ (d, $J=1.6$ $\mathrm{Hz}, 1 \mathrm{H}), 7.55$ (s, 1H), 7.39 (t, $J=7.6 \mathrm{~Hz}, 1 \mathrm{H}), 7.80(\mathrm{~d}, J=8.0 \mathrm{~Hz}, 1 \mathrm{H}), 7.16(\mathrm{~d}, J=7.2 \mathrm{~Hz}$, $1 \mathrm{H}), 3.67(\mathrm{~s}, 3 \mathrm{H}), 2.41(\mathrm{~s}, 3 \mathrm{H}), 2.40(\mathrm{~s}, 3 \mathrm{H}) ;{ }^{13} \mathrm{C} \mathrm{NMR}\left(100 \mathrm{MHz}, \mathrm{CDCl}_{3}\right): \delta$ 169.6, 150.7, 146.0, 139.0, 136.0, 135.7, 135.2, 133.2, 131.9, 130.2, 129.7, 127.2, 52.1, 19.7, 19.6. GCMS, $\mathrm{m} / \mathrm{z}$ (\% relative intensity, ion): $277\left[\mathrm{M}+2^{+}\right](11), 275\left[\mathrm{M}^{+}\right]$(34), 260 (4), 244 (100), 208 (9), 180 (13), 166 (9), 152 (16), 139 (5), 115 (6), 76 (7). IR: $\tilde{v}=1725,1591,1387,1166,1103,956$.

\section{Methyl 2-(6-chloropyridin-3-yl)-6-ethylbenzoate (3t)}<smiles>CCOC(=O)c1c(C)cccc1-c1ccc(Cl)nc1</smiles>

Compound 3t was prepared following the general procedure (using EtI instead of MeI for esterification), starting from 2-methoxybenzoic acid (35 mg, $0.25 \mathrm{mmol}$ ) and 5-bromo-2chloropyridine $(71.6 \mathrm{mg}, 0.375 \mathrm{mmol})$. After purification by column chromatography (ethyl acetate $/$ hexane $=5: 1)$, 3t was obtained as yellow liquid ( $78 \%$ isolated yield). ${ }^{1} \mathrm{H}$ NMR $\left(400 \mathrm{MHz}, \mathrm{CDCl}_{3}\right): \delta 8.37(\mathrm{~d}, J=2.4 \mathrm{~Hz}, 1 \mathrm{H}), 7.64(\mathrm{dd}, J=8.0 \mathrm{~Hz}, 2.4 \mathrm{~Hz}, 1 \mathrm{H}), 7.39-7.32$ (m, 2H), 7.27 (d, $J=7.6 \mathrm{~Hz}, 2 \mathrm{H}), 7.14$ (d, $J=7.2 \mathrm{~Hz}, 1 \mathrm{H}), 4.12$ (q, $J=7.2 \mathrm{~Hz}, 2 \mathrm{H}), 2.40$ (s, $3 \mathrm{H}), 1.07$ (t, $J=7.2 \mathrm{~Hz}, 3 \mathrm{H}) ;{ }^{13} \mathrm{C} \mathrm{NMR}\left(100 \mathrm{MHz}, \mathrm{CDCl}_{3}\right): \delta 168.8,150.4,148.8,138.5,136.0$, $135.5,135.0,133.4,130.3,129.6,127.1,123.6,61.1,19.6,13.8$. GCMS, m/z (\% relative intensity, ion): $277\left[\mathrm{M}+2^{+}\right]$(10), 275[M $\left.\mathrm{M}^{+}\right]$(29), 246 (9), 230 (100), 219 (5), 203 (7), 194 (9), 166 (29), 139 (22), 115 (9), 89 (5). IR: $\tilde{v}=2982,2361,1720,1589,1450,1261,1126,1088$, $1065,1023,791 \mathrm{~cm}^{-1}$ 


\section{Methyl 2-(2-chloropyridin-4-yl)-6-methylbenzoate (3u)}<smiles>COC(=O)c1c(C)cccc1-c1ccnc(Cl)c1</smiles>

Compound 3u was prepared following procedure B, starting from 2-methoxybenzoic acid (35 $\mathrm{mg}, 0.25 \mathrm{mmol})$ and 4-bromo-2-chloropyridine $(71.6 \mathrm{mg}, 0.375 \mathrm{mmol})$. After purification by column chromatography (ethyl acetate / hexane $=5: 1), \mathbf{3 x}$ was obtained as brown oil $(42.4 \mathrm{mg}$, $65 \%$ isolated yield). ${ }^{1} \mathrm{H}$ NMR $\left(400 \mathrm{MHz}, \mathrm{CDCl}_{3}\right): \delta 8.39(\mathrm{~d}, J=4.8 \mathrm{~Hz}, 1 \mathrm{H}), 7.40(\mathrm{t}, J=7.6$ $\mathrm{Hz}, 1 \mathrm{H}), 7.33-7.30(\mathrm{~m}, 2 \mathrm{H}), 7.20-7.17(\mathrm{~m}, 2 \mathrm{H}), 3.65(\mathrm{~s}, 3 \mathrm{H}), 2.41(\mathrm{~s}, 3 \mathrm{H}) ;{ }^{13} \mathrm{C} \mathrm{NMR}$ $\left(100 \mathrm{MHz}, \mathrm{CDCl}_{3}\right): \delta 169.2,151.9,151.7,149.4,136.4,136.1,132.6,131.0,129.9,126.6$, 123.5, 121.9, 52.1, 19.7. GCMS, m/z (\% relative intensity, ion): $263\left[\mathrm{M}+2^{+}\right](18), 261\left[\mathrm{M}^{+}\right](52)$, 230 (100), 202 (8), 194 (17), 166 (21), 139 (26), 115 (10), 89 (5). IR: $\tilde{v}=2928,2361,1724$, $1589,1535,1373,1265,1123,1096,1069,791,768$.

\section{Methyl 2-(6-chloro-5-fluoropyridin-3-yl)-6-methylbenzoate (3v)}<smiles>COC(=O)c1c(N)cccc1-c1cnc(Cl)c(F)c1</smiles>

Compound $3 \mathbf{v}$ was prepared following the general procedure, starting from 2-methoxybenzoic acid (35 mg, $0.25 \mathrm{mmol}$ ) and 5-bromo-2-chloro-3-fluoropyridine (78.4 mg, $0.375 \mathrm{mmol}$ ). After purification by column chromatography (ethyl acetate / hexane $=5: 1$ ), 3v was obtained as yellow liquid (50.9 mg, 73\% isolated yield). ${ }^{1} \mathrm{H}$ NMR (400 MHz, $\left.\mathrm{CDCl}_{3}\right): \delta 8.20$ (d, $J=1.2$ $\mathrm{Hz}, 1 \mathrm{H}), 7.49(\mathrm{dd}, J=8.8 \mathrm{~Hz}, 2 \mathrm{~Hz}, 1 \mathrm{H}), 7.41(\mathrm{t}, J=8.0 \mathrm{~Hz}, 1 \mathrm{H}), 7.31(\mathrm{~d}, J=7.6 \mathrm{~Hz}, 1 \mathrm{H}), 7.16$ $(\mathrm{d}, J=7.6 \mathrm{~Hz}, 1 \mathrm{H}), 3.69$ (s, 3H), 2.41 (s, 3H); ${ }^{13} \mathrm{C} \mathrm{NMR}\left(100 \mathrm{MHz}, \mathrm{CDCl}_{3}\right): 169.2,154.2$ (d, $J$ $=130.5 \mathrm{~Hz}, 1 \mathrm{C}), 143.8(\mathrm{~d}, J=5 \mathrm{~Hz}, 1 \mathrm{C}), 137.95(\mathrm{~d}, J=9.5 \mathrm{~Hz}, 1 \mathrm{C}), 137.5$ (d, $J=2 \mathrm{~Hz}, 1 \mathrm{C})$, 136.4, 133.8, 133.1, 130.8, 129.9, 127.1, 124.2 (d, $J=19 \mathrm{~Hz}, 1 \mathrm{C}), 52.2,19.7 .{ }^{19} \mathrm{~F}$ NMR (376 $\mathrm{MHz}, \mathrm{CDCl}_{3}$ ): $\delta-56.33(\mathrm{~d}, J=7.5 \mathrm{~Hz}, 1 \mathrm{~F}) . \mathrm{GCMS}, \mathrm{m} / \mathrm{z}$ (\% relative intensity, ion): $281\left[\mathrm{M}+2^{+}\right]$ (9), $279\left[\mathrm{M}^{+}\right]$(28), 250 (36), 248 (100), 184 (20), 158 (10), 91 (5). IR: $\tilde{v}=2951,2361,1724$, 1593, 1439, 1393, 1265, 1126, 1103, 1076, 791.

Methyl 2-(5-bromo-6-chloropyridin-3-yl)-6-methylbenzoate (3w) 


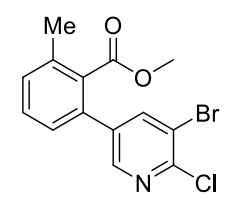

Compound 3w was prepared following the general procedure, starting from 2-methoxybenzoic acid (35 mg, $0.25 \mathrm{mmol}$ ) and 3,5-dibromo-2-chloropyridine (100.9 mg, $0.375 \mathrm{mmol})$. After purification by column chromatography (ethyl acetate / hexane $=5: 1$ ), $\mathbf{3 w}$ was obtained as yellow solid (46.6 mg, 55\% isolated yield). m.p. $=87-88^{\circ} \mathrm{C} .{ }^{1} \mathrm{H} \mathrm{NMR}\left(400 \mathrm{MHz}, \mathrm{CDCl}_{3}\right): \delta$ $8.32(\mathrm{~d}, J=1.6 \mathrm{~Hz}, 1 \mathrm{H}), 7.94(\mathrm{~d}, J=2.0 \mathrm{~Hz}, 1 \mathrm{H}), 7.40(\mathrm{t}, J=8.4 \mathrm{~Hz}, 1 \mathrm{H}), 7.30$ (d, $J=7.6 \mathrm{~Hz}$, $1 \mathrm{H}), 7.16(\mathrm{~d}, J=7.6 \mathrm{~Hz}, 1 \mathrm{H}), 3.70(\mathrm{~s}, 3 \mathrm{H}), 2.40(\mathrm{~s}, 3 \mathrm{H}),{ }^{13} \mathrm{C} \mathrm{NMR}\left(100 \mathrm{MHz}, \mathrm{CDCl}_{3}\right): \delta 169.2$, 149.7, 146.9, 141.6, 136.8, 136.4, 133.7, 133.1, 130.8, 129.9, 127.1, 119.7, 52.2, 19.8. GCMS,

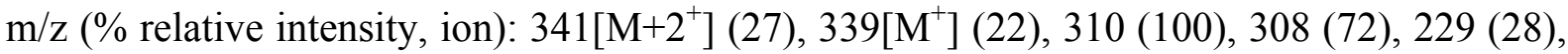
201 (11), 166 (29), 139 (20), 115 (5), 82 (10). IR: $\tilde{v}=2928,2360,1713,1269$.

\section{Methyl 3-methyl-3'-(pyrrolidine-1-carbonyl)biphenyl-2-carboxylate (3x)}

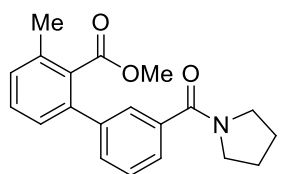

Compound $\mathbf{3 x}$ was prepared following the general procedure, starting from 2-methoxybenzoic acid (35 mg, $0.25 \mathrm{mmol}$ ) and (3-iodophenyl)(pyrrolidin-1-yl)methanone (112.9 mg, $0.375 \mathrm{mmol}$ ). After purification by column chromatography (ethyl acetate / hexane $=5: 1$ ), $\mathbf{3 x}$ was obtained as light yellow solid (76.8 mg, 95\% isolated yield). m.p. $=135-137^{\circ} \mathrm{C} .{ }^{1} \mathrm{H}$ NMR $\left(400 \mathrm{MHz}, \mathrm{CDCl}_{3}\right): \delta 7.49-7.47(\mathrm{~m}, 2 \mathrm{H}), 7.33(\mathrm{~d}, J=8.0 \mathrm{~Hz}, 1 \mathrm{H}), 7.19(\mathrm{dd}, J=12.0 \mathrm{~Hz}, 8.0$ $\mathrm{Hz}, 2 \mathrm{H}), 3.62$ (t, $J=8.0 \mathrm{~Hz}, 2 \mathrm{H}), 3.57$ (s, 3H), 3.41 (t, $J=8.0 \mathrm{~Hz}, 2 \mathrm{H}), 2.37$ (s, 3H), 1.96-1.90 (m, 2H), 1.88-1.83 (m, 2H); ${ }^{13} \mathrm{C}$ NMR (100 MHz, $\left.\mathrm{CDCl}_{3}\right): \delta 170.0,169.2,140.7,139.2,137.3$, 135.4, 133.0, 129.4, 129.4, 129.3, 128.2, 127.1, 126.8, 126.1, 51.8, 49.5, 46.1, 26.3, 24.3, 19.6. MS (APCI+), m/z: 323.85 [M+H'] IR: $\tilde{v}=2951,1724,1620,1431,1269,1119,1069,748$.

\section{3'-(Diethylcarbamoyl)-3-methylbiphenyl-2-carboxylic acid (3y)}

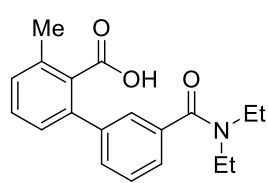

Compound 3y was prepared following the general procedure, starting from 2-methoxybenzoic acid (35 mg, $0.25 \mathrm{mmol}$ ) and 3-bromo- $N, N$-diethylbenzamide (95.6 mg, $0.375 \mathrm{mmol}$ ). After the 
reaction was complete, the reaction mixture was allowed to cool to rt. The reaction mixture was diluted with ethyl acetate $(20 \mathrm{~mL})$ and extracted with aq. $\mathrm{NaOH}$ solution $(3 \times 20 \mathrm{~mL})$. The aq. basic phase was acidified with $\mathrm{HCl}(\mathrm{pH} 1-2)$ and afterwards extracted with ethyl acetate $(3 \times$ $20 \mathrm{~mL}$ ). The combined organic layers were dried over $\mathrm{MgSO}_{4}$, filtered, and the volatiles were removed under reduced pressure. The residue was purified by column chromatography $\left(\mathrm{SiO}_{2}\right.$, ethyl acetate/hexane $=5: 1,1 \%$ fomic acid) yielding $\mathbf{3 y}$ as beige solid $(50.9 \mathrm{mg}, 65 \%$ isolated yield). ${ }^{1} \mathrm{H}$ NMR (400 MHz, $\left.\mathrm{CDCl}_{3}\right): \delta .7 .43-7.31(\mathrm{~m}, 4 \mathrm{H}), 7.29(\mathrm{~d}, J=4.4 \mathrm{~Hz}, 1 \mathrm{H}), 7.20$ (d, $J$ $=7.6 \mathrm{~Hz}, 1 \mathrm{H}), 7.15(\mathrm{~d}, J=7.6 \mathrm{~Hz}, 1 \mathrm{H}), 3.49(\mathrm{~d}, J=5.6 \mathrm{~Hz}, 2 \mathrm{H}), 3.24(\mathrm{~d}, J=6.0 \mathrm{~Hz}, 2 \mathrm{H}), 2.38$ (s, 3H), 1.19-1.06 (m, 6H); ${ }^{13} \mathrm{C}$ NMR (100 MHz, $\left.\mathrm{CDCl}_{3}\right): \delta 172.1,171.5,141.1,138.9,136.3$, $135.4,133.4,129.4,129.2,128.5,127.1,126.2,125.1,43.5,39.5,19.8,14.1,12.8$. MS (APCI+), m/z: $312.85\left[\mathrm{M}+\mathrm{H}^{+}\right] . \mathrm{IR}: \tilde{v}=2970,2928,2160,1717,1620,1543,1273,1123,768$.

\section{Methyl 4'-(isopropylcarbamoyl)-3-methylbiphenyl-2-carboxylate (3z)}

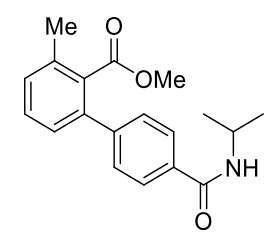

Compound $\mathbf{3 z}$ was prepared following the general procedure, starting from 2-methoxybenzoic acid (35 mg, $0.25 \mathrm{mmol}$ ) and 4-bromo- $N$-isopropylbenzamide (90.4 mg, $0.375 \mathrm{mmol}$ ). After purification by column chromatography (ethyl acetate $/$ hexane $=5: 1$ ), 3z was obtained as white solid (42.3 mg, 54\% isolated yield). m.p. $=170-172{ }^{\circ} \mathrm{C} .{ }^{1} \mathrm{H}$ NMR $\left(400 \mathrm{MHz}, \mathrm{CDCl}_{3}\right): \delta 7.78$ $(\mathrm{d}, J=8.0 \mathrm{~Hz}, 2 \mathrm{H}), 7.38-7.36(\mathrm{~m}, 2 \mathrm{H}), 7.34(\mathrm{t}, J=8.0 \mathrm{~Hz}, 1 \mathrm{H}), 7.22(\mathrm{~d}, J=8.0 \mathrm{~Hz}, 1 \mathrm{H}), 7.16$ $(\mathrm{d}, J=8.0 \mathrm{~Hz}, 1 \mathrm{H}), 7.32-7.28(\mathrm{~m}, 2 \mathrm{H}), 6.20(\mathrm{~d}, J=8.0 \mathrm{~Hz}, 1 \mathrm{H}), 4.29(\mathrm{dt}, J=12.0 \mathrm{~Hz}, 8.0 \mathrm{~Hz}$, $1 \mathrm{H}), 3.56(\mathrm{~s}, 3 \mathrm{H}), 2.38(\mathrm{~s}, 3 \mathrm{H}), 1.25(\mathrm{~d}, J=8.0 \mathrm{~Hz}, 6 \mathrm{H}) ;{ }^{13} \mathrm{C} \mathrm{NMR}\left(100 \mathrm{MHz}, \mathrm{CDCl}_{3}\right): \delta 169.9$, 166.3, 143.8, 139.0, 135.6, 133.7, 132.9, 129.6, 129.5, 128.2, 127.0, 126.8, 51.9, 41.8, 22.7, 19.6. MS (APCI+), m/z: $312.85\left[\mathrm{M}+\mathrm{H}^{+}\right]$. IR: $\tilde{v}=2967,2928,2160,1705,1574,1458,1254$, 787.

\section{Methyl 4-(trifluoromethyl)biphenyl-2-carboxylate (4ba)}

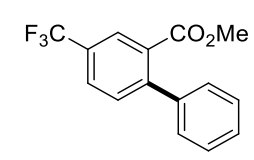

Compound 4ba was prepared following the general procedure, starting from 3(trifluoromethyl)benzoic acid $(47.5 \mathrm{mg}, 0.25 \mathrm{mmol})$ and phenyl iodide $(76.5 \mathrm{mg}, 0.375 \mathrm{mmol})$. 
After purification by column chromatography (ethyl acetate / hexane =10:1), 4ba was obtained as beige solid (54.6 mg, 78\% isolated yield). ${ }^{1} \mathrm{H}$ NMR (400 MHz, $\left.\mathrm{CDCl}_{3}\right): \delta 8.11(\mathrm{~s}, 1 \mathrm{H}), 7.79$ $(\mathrm{d}, J=8.0 \mathrm{~Hz}, 1 \mathrm{H}), 7.52(\mathrm{~d}, J=8.0 \mathrm{~Hz}, 1 \mathrm{H}), 7.45-7.42(\mathrm{~m}, 3 \mathrm{H}), 7.33-7.31(\mathrm{~m}, 2 \mathrm{H}), 3.68(\mathrm{~s}$, $3 \mathrm{H}) .{ }^{13} \mathrm{C} \mathrm{NMR}\left(100 \mathrm{MHz}, \mathrm{CDCl}_{3}\right): \delta 167.8,146.0,139.9,131.4,131.3,129.6$ (q, $\left.J=26 \mathrm{~Hz}\right)$, 128.2, 128.1, 128.0, 127.8 (q, $J=3.6 \mathrm{~Hz}), 126.9$ (q, $J=217 \mathrm{~Hz}), 126.7$ (q, $J=3.9 \mathrm{~Hz}), 52.3$. ${ }^{19} \mathrm{~F}$ NMR (376 MHz, $\left.\mathrm{CDCl}_{3}\right): \delta 0.23$ (s, 3F). GCMS, m/z (\% relative intensity, ion): $280\left[\mathrm{M}^{+}\right]$ (46), 261 (5), 249 (100), 201 (29), 152 (26), 125. IR: $\tilde{v}=2955,2361,1728,1335,1242,1169$, $1123,1084,768$.

\section{Methyl 4-bromobiphenyl-2-carboxylate (4ca) [CAS: 493028-83-4]}<smiles>CC(=O)c1cc(Br)ccc1-c1ccccc1</smiles>

Compound 4ca was prepared following the general procedure, starting from 3-bromobenzoic acid (50.0 mg, $0.25 \mathrm{mmol}$ ) and phenyl iodide $(76.5 \mathrm{mg}, 0.375 \mathrm{mmol})$. After purification by column chromatography (ethyl acetate / hexane $=10: 1$ ), 4ca was obtained as colorless liquid (45.1 mg, 62\% isolated yield). ${ }^{1} \mathrm{H}$ NMR (400 MHz, $\left.\mathrm{CDCl}_{3}\right): \delta 7.99$ (d, $\left.J=2.0 \mathrm{~Hz}, 1 \mathrm{H}\right), 7.67$ $(\mathrm{dd}, J=8.4 \mathrm{~Hz}, 2.0 \mathrm{~Hz}, 1 \mathrm{H}), 7.44-7.38(\mathrm{~m}, 3 \mathrm{H}), 7.31-7.28(\mathrm{~m}, 3 \mathrm{H}), 3.67(\mathrm{~s}, 3 \mathrm{H}) ;{ }^{13} \mathrm{C} \mathrm{NMR}$ $\left(100 \mathrm{MHz}, \mathrm{CDCl}_{3}\right): \delta 167.6,141.4,140.1,134.2,132.6,132.4,132.3,128.2,127.6,121.01$, 52.2. GCMS, m/z (\% relative intensity, ion): 292[M+2 $](61), 290\left[\mathrm{M}^{+}\right](69), 261(66), 259(64)$, 180 (55),152 (100), 126 (12), 99 (9), 76 (32). IR: $\tilde{v}=2951,1720,1435,1277,1238,1142,1092$, 760,698 .

\section{Methyl 4-chlorobiphenyl-2-carboxylate (4da) [CAS: 1092775-67-1] ${ }^{6}$}<smiles>CC(=O)c1cc(Cl)ccc1-c1ccccc1</smiles>

Compound 4da was prepared following procedure B, starting from 3-chlorobenzoic acid (39 $\mathrm{mg}, 0.25 \mathrm{mmol})$ and phenyl iodide $(76.5 \mathrm{mg}, 0.375 \mathrm{mmol})$. After purification by column chromatography (ethyl acetate / hexane $=10: 1)$, 4da was obtained as yellow liquid $(41.8 \mathrm{mg}$, $68 \%$ isolated yield). ${ }^{1} \mathrm{H}$ NMR (400 MHz, $\left.\mathrm{CDCl}_{3}\right): \delta 7.82(\mathrm{~d}, J=2.0 \mathrm{~Hz}, 1 \mathrm{H}), 7.50(\mathrm{dd}, J=8.4$ $\mathrm{Hz}, 2.0 \mathrm{~Hz}, 1 \mathrm{H}), 7.42-7.37(\mathrm{~m}, 3 \mathrm{H}), 7.32$ (d, $J=8.0 \mathrm{~Hz}, 1 \mathrm{H}), 7.28(\mathrm{dd}, J=8.0 \mathrm{~Hz}, 1.2 \mathrm{~Hz}$,

\footnotetext{
${ }^{5}$ Wang, Y.; Gulevich, A. V.; Gevorgyan, V. Chem. - Eur. J., 2013, 19, 15836-15840.

${ }^{6}$ Ramirez, N. P.; Bosque, I.; Gonzalez-Gomez, J. C., Org. Lett., 2015, 17, 4550-4553.
} 
2H), $3.65(\mathrm{~s}, 3 \mathrm{H}) ;{ }^{13} \mathrm{C} \mathrm{NMR}\left(100 \mathrm{MHz}, \mathrm{CDCl}_{3}\right): \delta 167.8,140.9,140.1,133.2,132.1,132.0$, 131.2, 129.7, 128.2, 128.1, 127.5, 52.2. GCMS, m/z (\% relative intensity, ion): $248\left[\mathrm{M}+2^{+}\right](26)$, 246[M $\left.\mathrm{M}^{+}\right]$(68), 215 (95), 152 (100), 76 (18). IR: $\tilde{v}=2951,2361,1720,1435,1277,1238,1088$, $764,698$.

\section{Methyl 4-methylbiphenyl-2-carboxylate (4ea) [CAS: 152620-33-2] ${ }^{5}$}<smiles>CC(=O)c1cc(C)ccc1-c1ccccc1</smiles>

Compound 4ea was prepared following procedure B, starting from 3-methylbenzoic acid (35 $\mathrm{mg}, 0.25 \mathrm{mmol})$ and phenyl iodide $(76.5 \mathrm{mg}, 0.375 \mathrm{mmol})$. After purification by column chromatography (ethyl acetate / hexane $=10: 1)$, 4ea was obtained as colorless oil $(53.1 \mathrm{mg}$, 94\% isolated yield). ${ }^{1} \mathrm{H}$ NMR (400 MHz, $\left.\mathrm{CDCl}_{3}\right): \delta 7.65(\mathrm{~s}, 1 \mathrm{H}), 7.42-7.39(\mathrm{~m}, 1 \mathrm{H}), 7.38$ 7.27 (m, 6H), 3.64 (s, 3H), $2.43(\mathrm{~s}, 3 \mathrm{H}) ;{ }^{13} \mathrm{C} \mathrm{NMR}\left(100 \mathrm{MHz}, \mathrm{CDCl}_{3}\right): \delta 169.3,141.2,139.6$, 137.0, 132.0, 130.61, 130.58, 130.2, 128.3, 128.0, 127.0, 51.8, 20.9. GCMS, m/z (\% relative intensity, ion): $226\left[\mathrm{M}^{+}\right](100), 195(95), 165$ (54), 152 (64), 139 (7), 115 (9). IR: $\tilde{v}=2947,2361$, $1717,1435,1296,1204,1088,764$.

\section{Methyl 4-acetylbiphenyl-2-carboxylate (4fa) [CAS: 537715-93-8] ${ }^{7}$}<smiles>CC(=O)c1ccc(-c2ccccc2)c(C(C)=O)c1</smiles>

Compound $4 \mathbf{f a}$ was prepared following procedure B, starting from 3-acetylbenzoic acid (41 mg, $0.25 \mathrm{mmol}$ ) and phenyl iodide $(76.5 \mathrm{mg}, 0.375 \mathrm{mmol})$. After purification by column chromatography (ethyl acetate / hexane $=10: 1)$, 4fa was obtained as colorless oil (63.5 mg, $99 \%$ isolated yield). ${ }^{1} \mathrm{H}$ NMR (400 MHz, $\left.\mathrm{CDCl}_{3}\right): \delta 8.39$ (d, $\left.J=1.6 \mathrm{~Hz}, 1 \mathrm{H}\right), 8.10$ (dd, $J=8.0$ Hz, 1.6 Hz, 1H), 7.49 (d, J=8.4 Hz, 1H), $7.44-7.39$ (m, 3H), $7.33-7.31$ (m, 2H), 3.67 (s, 3H), 2.66 (s, 3H); ${ }^{13} \mathrm{C} \mathrm{NMR}\left(100 \mathrm{MHz}, \mathrm{CDCl}_{3}\right): \delta 196.8,168.3,146.8,140.1,135.7,131.2$, 131.1, 130.6, 129.9, 128.2, 128.1, 127.9, 52.2, 26.6. GCMS, m/z (\% relative intensity, ion): 254[M $\left.\mathrm{M}^{+}\right]$(51), 239 (100), 223 (12), 196 (5), 152 (18), 139 (7), 76 (4). IR: $\tilde{v}=2951,2361,1721$, $1686,1231,1096,768,702$.

${ }^{7}$ Takasugi, H.; Inoue, Y.; Yoshikazu, T.; etc. PCT Int. Appl., 2003045921, 05 Jun 2003. 
Methyl 4-iodobiphenyl-2-carboxylate (4ga) [CAS: 69240-47-7] ${ }^{8}$<smiles>COC(=O)c1cc(I)ccc1-c1ccccc1</smiles>

Compound 4ga was prepared following the general procedure, starting from 3-iodobenzoic acid (62 $\mathrm{mg}, 0.25 \mathrm{mmol}$ ) and phenyl iodide $(76.5 \mathrm{mg}, 0.375 \mathrm{mmol})$. After purification by column chromatography (ethyl acetate / hexane =10: 1), 4ga was obtained as yellow oil $(42.2 \mathrm{mg}, 50 \%$ isolated yield). ${ }^{1} \mathrm{H} \mathrm{NMR}\left(400 \mathrm{MHz}, \mathrm{CDCl}_{3}\right): \delta 8.16(\mathrm{~d}, J=1.6 \mathrm{~Hz}, 1 \mathrm{H}), 7.85(\mathrm{dd}, J=8.0 \mathrm{~Hz}$, $2.0 \mathrm{~Hz}, 1 \mathrm{H}), 7.43-7.37(\mathrm{~m}, 3 \mathrm{H}), 7.28(\mathrm{dd}, J=7.6 \mathrm{~Hz}, 1.6 \mathrm{~Hz}, 2 \mathrm{H}), 7.11(\mathrm{~d}, J=8.0 \mathrm{~Hz}, 1 \mathrm{H})$, $3.64(\mathrm{~s}, 3 \mathrm{H}) ;{ }^{13} \mathrm{C} \mathrm{NMR}\left(100 \mathrm{MHz}, \mathrm{CDCl}_{3}\right): \delta 167.5,141.9,140.13,140.10,138.4,132.5,132.4$, 128.1, 128.1, 127.6, 92.2, 52.2. GCMS, m/z (\% relative intensity, ion): $338\left[\mathrm{M}^{+}\right](100), 307(80)$, 196 (7), 180 (47), 152 (76), 139 (13), 102 (7), 76 (14). IR: $\tilde{v}=2947,1721,1466,1435,1277$, $1238,1088,760,698$.

\section{Methyl 2-phenyl-1-naphthoate (4ha) [CAS: 109251-89-0] ${ }^{9}$}

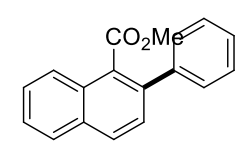

Compound 4 ha was prepared following the general procedure, starting from 1-naphthoic acid (43 $\mathrm{mg}, 0.25 \mathrm{mmol}$ ) and phenyl iodide $(76.5 \mathrm{mg}, 0.375 \mathrm{mmol})$. After purification by column chromatography (ethyl acetate / hexane $=10: 1)$, 4ha was obtained as yellow oil $(63.5 \mathrm{mg}, 97 \%$ isolated yield). ${ }^{1} \mathrm{H} \mathrm{NMR}\left(400 \mathrm{MHz}, \mathrm{CDCl}_{3}\right): \delta 7.98$ (dd, $\left.J=12 \mathrm{~Hz}, 8.4 \mathrm{~Hz}, 2 \mathrm{H}\right), 7.91(\mathrm{~d}, J=$ $8.0 \mathrm{~Hz}, 1 \mathrm{H}), 7.62-7.58(\mathrm{~m}, 1 \mathrm{H}), 7.57-7.45(\mathrm{~m}, 6 \mathrm{H}), 7.42-7.39(\mathrm{~m}, 1 \mathrm{H}), 3.72(\mathrm{~s}, 3 \mathrm{H})$. ${ }^{13} \mathrm{C} \mathrm{NMR}\left(100 \mathrm{MHz}, \mathrm{CDCl}_{3}\right): \delta 170.0,140.9,138.0,132.3,129.92,129.89,128.5,128.4(2 \mathrm{C})$, $128.1,127.6,127.42,127.36,126.3,125.0,52.1$. GCMS, $\mathrm{m} / \mathrm{z}$ (\% relative intensity, ion): 262[M $\left[\mathrm{M}^{+}\right]$(67), 231 (100), 202 (38), 101 (23), 88 (7). IR: $\tilde{v}=2947,1721,1435,1231,1138,826$, $760,703$.

\section{Methyl 3-ethoxybiphenyl-2-carboxylate (4ia)}

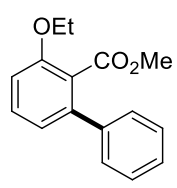

\footnotetext{
${ }^{8}$ Hawkins, A. F.; Jones, I.; Lewis, T., U.S. (1980), US 4242121 A 19801230.

${ }^{9}$ Zhao, Y.; Snieckus, V., Chem. Commun., 2016, 52, 1681-1684.
} 
Compound 4ia was prepared following the general procedure, starting from 2-ethoxybenzoic acid (42 $\mathrm{mg}, 0.25 \mathrm{mmol})$ and phenyl iodide $(76.5 \mathrm{mg}, 0.375 \mathrm{mmol})$. After purification by column chromatography (ethyl acetate / hexane $=10: 1)$, 4ia was obtained as yellow solid (61 mg, 95\% isolated yield). m.p. $=73-74{ }^{\circ} \mathrm{C} .{ }^{1} \mathrm{H}$ NMR $\left(400 \mathrm{MHz}, \mathrm{CDCl}_{3}\right): \delta 7.42-7.34(\mathrm{~m}$, $6 \mathrm{H}), 6.98(\mathrm{~d}, J=8.0 \mathrm{~Hz}, 1 \mathrm{H}), 6.93(\mathrm{~d}, J=8.0 \mathrm{~Hz}, 1 \mathrm{H}), 4.12$ (q, $J=8.0 \mathrm{~Hz}, 2 \mathrm{H}), 3.65(\mathrm{~s}, 3 \mathrm{H})$, $1.42(\mathrm{t}, J=7.2 \mathrm{~Hz}, 3 \mathrm{H}) ;{ }^{13} \mathrm{C} \mathrm{NMR}\left(100 \mathrm{MHz}, \mathrm{CDCl}_{3}\right): \delta 168.5,155.8,141.1,140.1,130.4$, $128.3,128.2,127.5,123.5,121.8,111.0,64.5,51.9,14.7$. GCMS, m/z (\% relative intensity, ion): $256\left[\mathrm{M}^{+}\right]$(98), 225 (87), 196 (100), 168 (96), 141 (27), 139 (73), 115 (27). IR: $\tilde{v}=2924$, $1728,1253,1165,1119,783$.

\section{Methyl 3-phenylbiphenyl-2-carboxylate (4ja) [CAS: 19832-92-9] ${ }^{10}$}<smiles>COC(=O)c1c(-c2ccccc2)cccc1-c1ccccc1</smiles>

Compound 4ja was prepared following the general procedure1, starting from biphenyl-2carboxylic acid (50 mg, $0.25 \mathrm{mmol})$ and phenyl iodide (76.5 $\mathrm{mg}, 0.375 \mathrm{mmol})$. After purification by column chromatography (ethyl acetate / hexane =10: 1), 4ja was obtained as white solid (65.5 mg, 91\% isolated yield). m.p. $=120-121{ }^{\circ} \mathrm{C} .{ }^{1} \mathrm{H} \mathrm{NMR}\left(400 \mathrm{MHz}, \mathrm{CDCl}_{3}\right)$ : $\delta 7.53(\mathrm{dd}, J=8.4 \mathrm{~Hz}, 7.6 \mathrm{~Hz}, 1 \mathrm{H}), 7.44-7.38(\mathrm{~m}, 12 \mathrm{H}), 3.41(\mathrm{~s}, 3 \mathrm{H}) ;{ }^{13} \mathrm{C} \mathrm{NMR}(100 \mathrm{MHz}$, $\left.\mathrm{CDCl}_{3}\right): \delta 169.8,140.5,140.3,132.8,129.3,128.8,128.3,128.3,127.5,51.7 . \mathrm{GCMS}, \mathrm{m} / \mathrm{z}(\%$

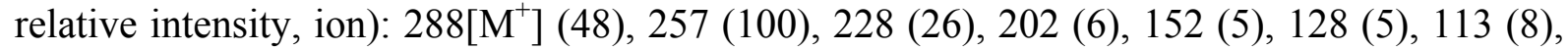
101 (7). IR: $\tilde{v}=2947,1724,1439,1281,1250,1119,748,702$.

\section{Methyl 3-phenylthiophene-2-carboxylate (4ka) [CAS: 21676-89-1] ${ }^{11}$}<smiles>CC(=O)c1sccc1-c1ccccc1</smiles>

Compound 4ka was prepared following the general procedure, starting from thiophene-2carboxylic acid (32 mg, $0.25 \mathrm{mmol}$ ) and phenyl iodide (76.5 mg, $0.375 \mathrm{mmol})$. After purification by column chromatography (ethyl acetate / hexane $=10: 1$ ), 4ka was obtained as white solid (44.1 mg, 81\% isolated yield). m.p. $=117-118{ }^{\circ} \mathrm{C} .{ }^{1} \mathrm{H} \mathrm{NMR}\left(400 \mathrm{MHz}, \mathrm{CDCl}_{3}\right)$ :

\footnotetext{
${ }^{10}$ Mroß, G.; Ladzik, S.; Reinke, H.; Spannenberg, A.; Fischer,C.; Langer, P., Synthesis, 2009, 2236-2248.

${ }^{11}$ Yang, J.; Liu, S.; Zheng, J.-F.; Zhou, J., Eur. J. Org. Chem., 2012, 3248-6259.
} 
$\delta 7.51(\mathrm{~d}, J=8.0 \mathrm{~Hz}, 1 \mathrm{H}), 7.48-7.46(\mathrm{~m}, 2 \mathrm{H}), 7.44-7.38(\mathrm{~m}, 3 \mathrm{H}), 7.10(\mathrm{~d}, J=5.2 \mathrm{~Hz}, 1 \mathrm{H})$, $3.78(\mathrm{~s}, 3 \mathrm{H}) ;{ }^{13} \mathrm{C} \mathrm{NMR}\left(100 \mathrm{MHz}, \mathrm{CDCl}_{3}\right): \delta 162.4,148.7,135.6,131.5,130.2,129.2,127.9$, 127.8, 126.9, 51.9. GCMS, m/z (\% relative intensity, ion): $218\left[\mathrm{M}^{+}\right](91), 187$ (85), 159 (18), 115 (100), 89 (17), 79 (12). IR: $\tilde{v}=2924,1705,1439,1281,1234,1119,1088,752$.

\section{Methyl 3-(6-chloropyridin-3-yl)thiophene-2-carboxylate (4kt)}

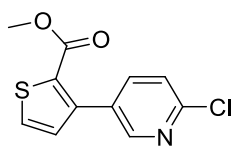

Compound 4kt was prepared following the general procedure, starting from thiophene-2carboxylic acid (32 mg, $0.25 \mathrm{mmol})$ and 5-bromo-2-chloropyridine (71.6 mg, $0.375 \mathrm{mmol}$ ). After purification by column chromatography (ethyl acetate / hexane $=5: 1$ ), 4kt was obtained as colorless solid (13.3 mg, 21\% isolated yield). m.p. $=149-151{ }^{\circ} \mathrm{C} .{ }^{1} \mathrm{H}$ NMR $(400 \mathrm{MHz}$, $\left.\mathrm{CDCl}_{3}\right): \delta 8.44(\mathrm{~d}, J=2.0 \mathrm{~Hz}, 1 \mathrm{H}), 7.79(\mathrm{dd}, J=8.0 \mathrm{~Hz}, 2.0 \mathrm{~Hz}, 1 \mathrm{H}), 7.58(\mathrm{~d}, J=5.2 \mathrm{~Hz}, 1 \mathrm{H})$, $7.37(\mathrm{~d}, J=8.4 \mathrm{~Hz}, 1 \mathrm{H}), 7.08(\mathrm{~d}, J=2.0 \mathrm{~Hz}, 1 \mathrm{H}), 3.80(\mathrm{~s}, 3 \mathrm{H}) ;{ }^{13} \mathrm{C} \mathrm{NMR}\left(100 \mathrm{MHz}, \mathrm{CDCl}_{3}\right)$ : $\delta 162.0,150.8,149.4,143.2,139.6,131.2,130.9,130.4,128.4,123.3,52.2$. GCMS, m/z (\% relative intensity, ion): $255\left[\mathrm{M}+2^{+}\right](38), 253\left[\mathrm{M}^{+}\right](100), 224$ (35), $222(91), 186(31), 159(22)$, 114 (20), 94 (12). IR: $\tilde{v}=1708,1463,1431,1243,1226,1080,892,833$.

\section{Methyl 2,6-dimethyl-4-phenylnicotinate (4la)}

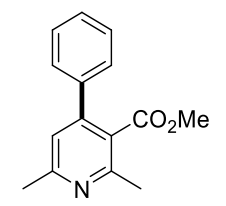

Compound 4la was prepared following the general procedure, starting from 2,6dimethylnicotinic acid (38 $\mathrm{mg}, 0.25 \mathrm{mmol})$ and phenyl iodide $(76.5 \mathrm{mg}, 0.375 \mathrm{mmol})$. After purification by column chromatography (ethyl acetate / hexane $=5: 1$ ), 4la was obtained as yellow solid (32.5 mg, 54\% isolated yield). m.p. $=31-32{ }^{\circ} \mathrm{C}{ }^{1} \mathrm{H}$ NMR $\left(400 \mathrm{MHz}, \mathrm{CDCl}_{3}\right): \delta$ $7.41-7.38(\mathrm{~m}, 3 \mathrm{H}), 7.35-7.33(\mathrm{~m}, 2 \mathrm{H}), 7.02(\mathrm{~s}, 1 \mathrm{H}), 3.60(\mathrm{~s}, 3 \mathrm{H}), 2.59(\mathrm{~s}, 3 \mathrm{H}), 2.57(\mathrm{~s}, 3 \mathrm{H}) ;{ }^{13} \mathrm{C}$ NMR (100 MHz, $\left.\mathrm{CDCl}_{3}\right): \delta$ 169.6, 158.8, 155.1, 148.3, 138.6, 128.5, 128.4, 127.7, 125.4, 121.1, 52.1, 24.4, 22.8. GCMS, m/z (\% relative intensity, ion): $241\left[\mathrm{M}^{+}\right]$(72), $210(100), 182$ (11), 167 (18), 139 (13), 115 (33). IR: $\tilde{v}=1727,1589,1266,1212,1137$.

\section{7-Methyl-6H-benzo[c]chromen-6-one (6a) [CAS: 106737-97-7] ${ }^{4}$}




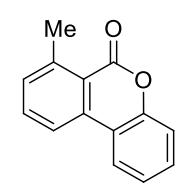

Compound 6a was prepared following the general procedure, starting from 2-methylbenzoic acid (35 mg, $0.25 \mathrm{mmol})$ and 2-iodophenol $(82.5 \mathrm{mg}, 0.375 \mathrm{mmol})$. After purification by column chromatography (ethyl acetate / hexane $=10: 1)$, 6a was obtained as white solid (49.8 mg, $95 \%$ isolated yield). m.p. $=101-102{ }^{\circ} \mathrm{C} .{ }^{1} \mathrm{H} \mathrm{NMR}\left(400 \mathrm{MHz}, \mathrm{CDCl}_{3}\right): \delta 7.96(\mathrm{q}, J=8.0$ $\mathrm{Hz}, 2 \mathrm{H}), 7.62$ (t, J=8.0 Hz, 2H), $7.44-7.40(\mathrm{~m}, 1 \mathrm{H}), 7.34$ (d, J=8.0 Hz, 1H), $7.29-7.25$ (m, 2H), 2.84 (s, 3H); ${ }^{13} \mathrm{C}$ NMR (100 MHz, $\left.\mathrm{CDCl}_{3}\right): \delta 160.3,151.2,144.3,136.0,133.8,132.1$, 130.1, 124.1, 122.9, 119.7, 119.6, 118.2, 117.2, 23.8. GCMS, m/z (\% relative intensity,

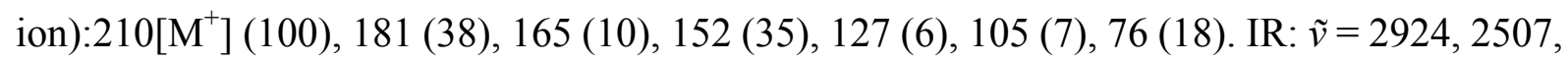
2160, 1717, 1462, 1246, 1207, 1065, 752.

\section{H-benzo[c]chromen-6-one (6b) [CAS: 2005-10-9 $]^{12}$}<smiles>O=c1oc2ccccc2c2ccccc12</smiles>

Compound $\mathbf{6 b}$ was prepared following the general procedure, starting from benzoic acid (31 $\mathrm{mg}, 0.25 \mathrm{mmol})$ and 2-iodophenol $(82.5 \mathrm{mg}, 0.375 \mathrm{mmol})$. After purification by column chromatography (ethyl acetate / hexane $=10: 1)$, $6 \mathbf{b}$ was obtained as white solid $(35.3 \mathrm{mg}, 72 \%$ isolated yield). m.p. $=93-94{ }^{\circ} \mathrm{C} .{ }^{1} \mathrm{H}$ NMR $\left(400 \mathrm{MHz}, \mathrm{CDCl}_{3}\right): \delta 8.38(\mathrm{~d}, J=8.0 \mathrm{~Hz}, 2 \mathrm{H})$, $8.10(\mathrm{~d}, J=8.0 \mathrm{~Hz}, 2 \mathrm{H}), 8.04(\mathrm{~d}, J=8.0 \mathrm{~Hz}, 1 \mathrm{H}), 7.81(\mathrm{t}, J=8.0 \mathrm{~Hz}, 2 \mathrm{H}), 7.57(\mathrm{t}, J=8.0 \mathrm{~Hz}$, 1H), $7.49-7.45(\mathrm{~m}, 1 \mathrm{H}), 7.36-7.30(\mathrm{~m}, 1 \mathrm{H}) ;{ }^{13} \mathrm{C} \mathrm{NMR}\left(100 \mathrm{MHz}, \mathrm{CDCl}_{3}\right): \delta 161.1,151.2$, $134.8,134.7,130.5,130.4,128.8,124.5,122.7,121.6,121.2,118.0,117.7$. GCMS, m/z (\% relative intensity, ion): $196\left[\mathrm{M}^{+}\right](100), 168$ (65), 139 (81), 113 (12), 98 (11), 84 (8). IR: $\tilde{v}=$ 2924, 2160, 1728, 1605, 1261, 1076, 1030, 745, 718.

\section{8-Acetyl-6H-benzo[c]chromen-6-one (6c) [CAS: 1447543-99-8] ${ }^{13}$}

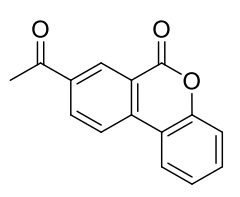

\footnotetext{
${ }^{12}$ Zhang, Z.; Gao, Y.; Liu, Y.; Li, J.; Xie, H.; Li, H.; Wang, W., Org. Lett., 2015, 17, 5492-5495.

${ }^{13}$ Luo, S.; Luo, F.-X; Zhang, X.-S.; Shi, Z.-J., Angew. Chem. Int. Ed., 2013, 52, 10598-10601.
} 
Compound 6c was prepared following the general procedure, starting from 3-acetylbenzoic acid (41 $\mathrm{mg}, 0.25 \mathrm{mmol})$ and 2-iodophenol $(82.5 \mathrm{mg}, 0.375 \mathrm{mmol})$. After purification by column chromatography (ethyl acetate / hexane $=10: 1)$, $6 \mathbf{c}$ was obtained as light yellow solid (51.7 mg, $92 \%$ isolated yield). m.p. $=185-186{ }^{\circ} \mathrm{C} .{ }^{1} \mathrm{H}$ NMR $\left(400 \mathrm{MHz}, \mathrm{CDCl}_{3}\right): \delta 8.87(\mathrm{~d}, J=1.6$ $\mathrm{Hz}, 1 \mathrm{H}), 8.38$ (dd, $J=8.4 \mathrm{~Hz}, 1.6 \mathrm{~Hz}, 1 \mathrm{H}), 8.17$ (d, $J=8.4 \mathrm{~Hz}, 1 \mathrm{H}), 8.06$ (d, $J=7.2 \mathrm{~Hz}, 1 \mathrm{H})$, $7.55-7.51(\mathrm{~m}, 1 \mathrm{H}), 7.38$ - $7.34(\mathrm{~m}, 2 \mathrm{H}), 2.69(\mathrm{~s}, 3 \mathrm{H}) ;{ }^{13} \mathrm{C} \mathrm{NMR}\left(100 \mathrm{MHz}, \mathrm{CDCl}_{3}\right): \delta 196.2$, $160.5,151.7,138.4,136.9,133.5,131.8,131.2,124.9,123.4,122.3,121.1,117.9,117.2$, 26.6. GCMS, m/z (\% relative intensity, ion): 238[ $\left.\mathrm{M}^{+}\right]$(9), 223 (17), 211 (100), 183 (17), 155 (26), 154 (61), 139 (13), 127 (23), 77 (16). IR: $\tilde{v}=2924,2160,1975,1717,1670,1605,1200,1115$, $1088,752$.

\section{8-Chloro-6H-benzo[c]chromen-6-one (6d)}<smiles>O=c1oc2ccccc2c2ccc(Cl)cc12</smiles>

Compound 6d was prepared following the general procedure, starting from 3-chlorobenzoic acid (39 $\mathrm{mg}, 0.25 \mathrm{mmol}$ ) and 2-iodophenol $(82.5 \mathrm{mg}, 0.375 \mathrm{mmol})$. After purification by column chromatography (ethyl acetate $/$ hexane $=10: 1), \mathbf{6 d}$ was obtained as brown solid (55.2 mg, 96\% isolated yield). m.p. $=174-175{ }^{\circ} \mathrm{C} .{ }^{1} \mathrm{H}$ NMR $\left(400 \mathrm{MHz}, \mathrm{CDCl}_{3}\right): \delta 8.30(\mathrm{~d}, J=2.0$ Hz, 1H), 8.02 (d, J=8.8 Hz, 2H), $7.98-7.96$ (m, 1H), 7.73 (dd, J=8.4 Hz, 2.0 Hz, 1H), 7.48 $(\mathrm{t}, J=7.6 \mathrm{~Hz}, 1 \mathrm{H}), 7.35-7.31(\mathrm{~m}, 2 \mathrm{H}) ;{ }^{13} \mathrm{C} \mathrm{NMR}\left(100 \mathrm{MHz}, \mathrm{CDCl}_{3}\right): \delta 159.9,151.0,135.0$, 134.9, 133.1, 130.8, 129.9, 124.8, 123.3, 122.7, 122.4, 117.8, 117.2. GCMS, m/z (\% relative intensity, ion): $232\left[\mathrm{M}+2^{+}\right](34), 230\left[\mathrm{M}^{+}\right](100), 202$ (23), 139 (51), 87 (7), 69 (9). IR: $\tilde{v}=2928$, $2160,1975,1717,1601,1308,826,745$.

\section{9-Bromo-6H-benzo[c]chromen-6-one (6e) [CAS: 1469912-47-7] $]^{14}$}<smiles>O=c1oc2ccccc2c2cc(Br)ccc12</smiles>

Compound 6e was prepared following the general procedure, starting from 4-bromobenzoic acid (50 mg, $0.25 \mathrm{mmol})$ and 2-iodophenol $(82.5 \mathrm{mg}, 0.375 \mathrm{mmol})$. After purification by column chromatography (ethyl acetate / hexane $=10: 1)$, 6e was obtained as white solid (59.6

\footnotetext{
${ }^{14}$ Zhou, J.; Han, P.; Xu, Y.-M.; Zhang, T.; Du, Z.-T., Heterocycles, 2013, 87, 1889-1896.
} 
mg, 87\% isolated yield). m.p. $=180-181{ }^{\circ} \mathrm{C} .{ }^{1} \mathrm{H}$ NMR $\left(400 \mathrm{MHz}, \mathrm{CDCl}_{3}\right): \delta 8.23-8.21(\mathrm{~m}$, 2H), $7.95(\mathrm{~d}, J=8.0 \mathrm{~Hz}, 1 \mathrm{H}), 7.67(\mathrm{dd}, J=8.4 \mathrm{~Hz}, 1.2 \mathrm{~Hz}, 1 \mathrm{H}), 7.52-7.48(\mathrm{~m}, 1 \mathrm{H}), 7.35$ $7.32(\mathrm{~m}, 2 \mathrm{H}) ;{ }^{13} \mathrm{C}$ NMR $\left(100 \mathrm{MHz}, \mathrm{CDCl}_{3}\right): \delta 160.5,151.5,136.3,132.1,131.2,130.6,124.9$, 124.7, 122.8, 119.9, 117.9, 116.8. GCMS, m/z (\% relative intensity, ion): $276\left[\mathrm{M}+2^{+}\right](96)$, 274[ $\left[\mathrm{M}^{+}\right]$(100), 246 (23), 248 (21), 195 (7), 167 (14), 139 (83), 113 (14), 98 (13). IR: $\tilde{v}=2928$, 2160, 1975, 1736, 1597, 1258, 1088, 1011, 745.

\section{7-(Methylamino)-6H-benzo[c]chromen-6-one (6f)}<smiles>CNc1cccc2c1c(=O)oc1ccccc12</smiles>

Compound 6f was prepared following the general procedure, starting from 2(methylamino)benzoic acid (37.8 $\mathrm{mg}, 0.25 \mathrm{mmol})$ and 2-iodophenol $(82.5 \mathrm{mg}, 0.375 \mathrm{mmol})$. After purification by column chromatography (ethyl acetate / hexane $=5: 1$ ), $6 \mathbf{f}$ was obtained as brown solid $\left(29.2 \mathrm{mg}, 52 \%\right.$ isolated yield). m.p. $=143-144{ }^{\circ} \mathrm{C} .{ }^{1} \mathrm{H}$ NMR $(400 \mathrm{MHz}$, $\left.\mathrm{CDCl}_{3}\right): \delta 8.49(\mathrm{~s}, 1 \mathrm{H}), 7.97(\mathrm{~d}, J=8.0 \mathrm{~Hz}, 1 \mathrm{H}), 7.57(\mathrm{t}, J=8.0 \mathrm{~Hz}, 1 \mathrm{H}), 7.42(\mathrm{t}, J=8.0 \mathrm{~Hz}$, 1H), 7.29-7.26 (m, 2H), $7.24(\mathrm{~d}, J=8.0 \mathrm{~Hz}, 1 \mathrm{H}), 6.67$ (d, $J=8.0 \mathrm{~Hz}, 1 \mathrm{H}), 2.97$ (d, $J=5.2 \mathrm{~Hz}$, $3 \mathrm{H}) ;{ }^{13} \mathrm{C} \mathrm{NMR}\left(100 \mathrm{MHz}, \mathrm{CDCl}_{3}\right): \delta 163.4,153.1,150.9,136.2,136.0,129.9,124.2,123.3$, 118.8, 117.2, 109.4, 107.2, 102.9, 29.7. GCMS, m/z (\% relative intensity, ion): $225\left[\mathrm{M}^{+}\right]$(100), 224 (51), 196 (21), 168 (20), 139 (24), 113 (9), 98 (5). IR: $\tilde{v}=2924,2160$, 1975, 1686, 1574, $1443,1250,1096,748$.

\section{8-Amino-6H-benzo[c]chromen-6-one (6g) [CAS: 27421-12-1 $]^{15}$}<smiles>Nc1ccc2c(c1)c(=O)oc1ccccc12</smiles>

Compound 6g was prepared following the general procedure, starting from 3-aminobenzoic acid (34.5 mg, 0.25mmol) and 2-iodophenol $(82.5 \mathrm{mg}, 0.375 \mathrm{mmol})$. After purification by column chromatography (ethyl acetate / hexane $=5: 1), \mathbf{6 g}$ was obtained as brown solid (20.6 $\mathrm{mg}, 39 \%$ isolated yield). m.p. $=168-170{ }^{\circ} \mathrm{C} .{ }^{1} \mathrm{H}$ NMR $\left(400 \mathrm{MHz}, \mathrm{CDCl}_{3}\right): \delta 8.58(\mathrm{~d}, J=$ $8.0 \mathrm{~Hz}, 1 \mathrm{H}), 7.93(\mathrm{~d}, J=8.0 \mathrm{~Hz}, 2 \mathrm{H}), 7.42-7.29(\mathrm{~m}, 4 \mathrm{H}), 7.16(\mathrm{~d}, J=8.0 \mathrm{~Hz}, 4 \mathrm{H}), 4.29(\mathrm{~s}, 2 \mathrm{H})$;

\footnotetext{
${ }^{15}$ Zhi, Li.; Ringgenber, J. D.; Edwards, J. P.; Tegley, C. M.; West, S. J.; Pio, B.; Motamedi, M.; Jones, T. K.; Marschke, K. B.; Mais, D. E.; Schrader, W. T., Bioorg. Med. Chem. Lett., 2003, 13, 2075-2078.
} 
${ }^{13} \mathrm{C} \mathrm{NMR}\left(100 \mathrm{MHz}, \mathrm{CDCl}_{3}\right): \delta$ 161.6, 150.4, 144.2, 129.0, 129.0, 125.1, 124.3, 124.0, 123.1, 122.0, 121.4, 118.9, 117.8. GCMS, m/z (\% relative intensity, ion): $211\left[\mathrm{M}^{+}\right]$(100), 183 (16), 154 (52), 127 (21), 102 (8), 91 (4), 77 (16). IR: $\tilde{v}=3332,1710,1425,1323,1284,1248,867$ (s).

\section{2,4-Dimethyl-5H-chromeno[3,4-c]pyridin-5-one (6h) [CAS: 104431-78-9] ${ }^{16}$}

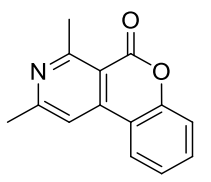

Compound 6h was prepared following the general procedure, starting from 2,6dimethylnicotinic acid (37.8 mg, $0.25 \mathrm{mmol})$ and 2-iodophenol $(82.5 \mathrm{mg}, 0.375 \mathrm{mmol})$. After purification by column chromatography (ethyl acetate / hexane $=5: 1$ ), $\mathbf{6 h}$ was obtained as white solid (43.3 mg, 77\% isolated yield). m.p. $=198-200{ }^{\circ} \mathrm{C}{ }^{1} \mathrm{H} \mathrm{NMR}\left(400 \mathrm{MHz}, \mathrm{CDCl}_{3}\right): \delta$ $8.00(\mathrm{~d}, J=8.0 \mathrm{~Hz}, 1 \mathrm{H}), 7.64(\mathrm{~s}, 1 \mathrm{H}), 7.54(\mathrm{t}, J=8.0 \mathrm{~Hz}, 1 \mathrm{H}), 7.32(\mathrm{t}, J=8.0 \mathrm{~Hz}, 2 \mathrm{H}), 3.03(\mathrm{~s}$, $3 \mathrm{H}), 2.68(\mathrm{~s}, 3 \mathrm{H}) ;{ }^{13} \mathrm{C} \mathrm{NMR}\left(100 \mathrm{MHz}, \mathrm{CDCl}_{3}\right): \delta 164.2,162.5,159.6,152.6,143.0,132.4$, 124.4, 123.5, 117.5, 116.2, 112.5, 26.7, 25.2. GCMS, m/z (\% relative intensity, ion): $225\left[\mathrm{M}^{+}\right]$ (100), 197 (6), 156 (16), 127 (9), 115 (4), 102 (7), 77(4). IR: $\tilde{v}=2924,1720,1589,1250,1189$, $1051,1024,917$

\footnotetext{
${ }^{16}$ Winneker, R. C.; Wagner, M. M.; Singh, B., J. Med. Chem., 1990, 33, 129-132.
} 
hua2-157-h

Avance $400 \# 1$

h1_latest
SKSUR

Magnet Revived

Revised: 21 May 15

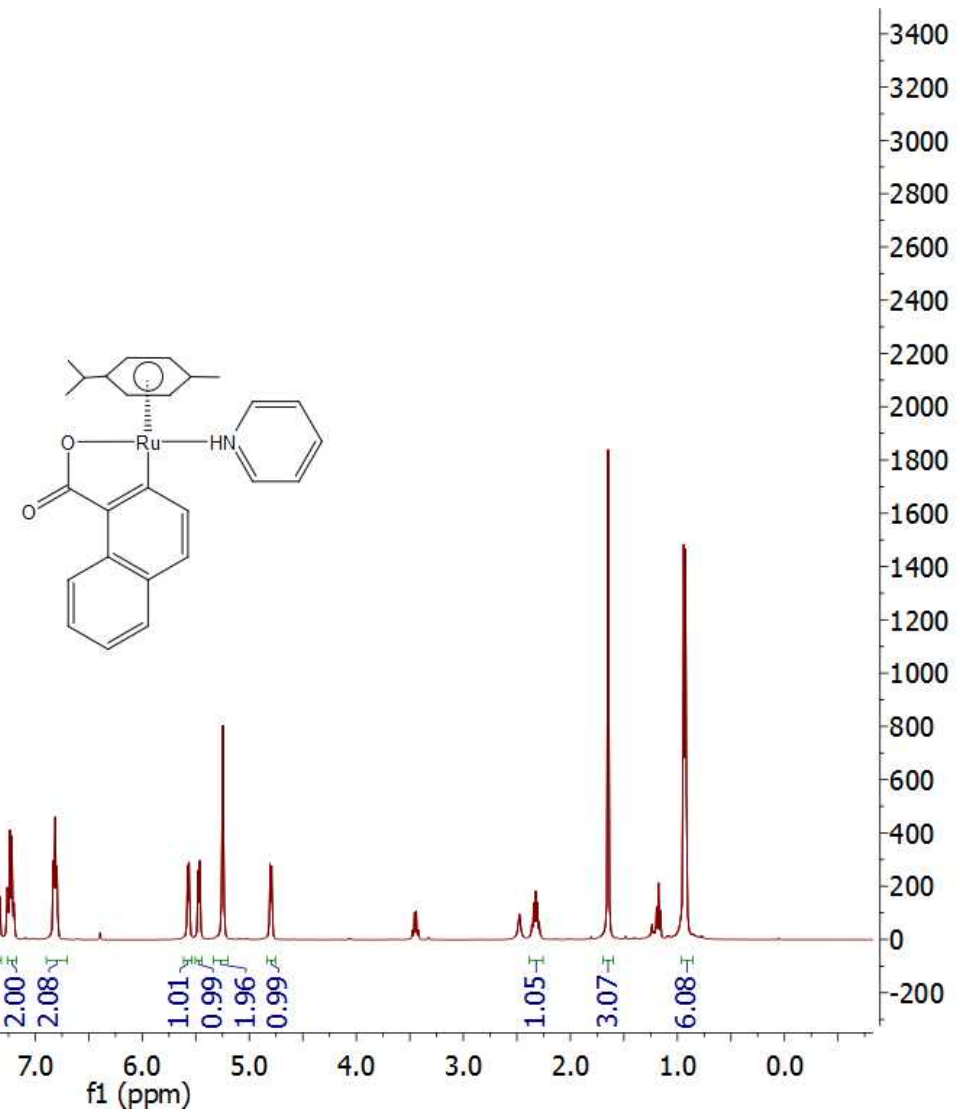

hua2-157-c N

\begin{tabular}{lll} 
Carbon NMR & c13 latest \\
SKSur & $-\infty$ & 0 \\
\hline
\end{tabular}

Plot Paper

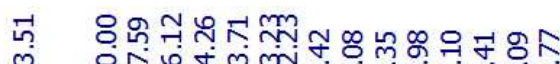

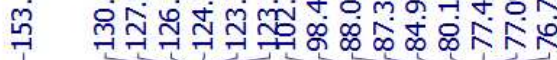

1 April 2011

ก่งก่

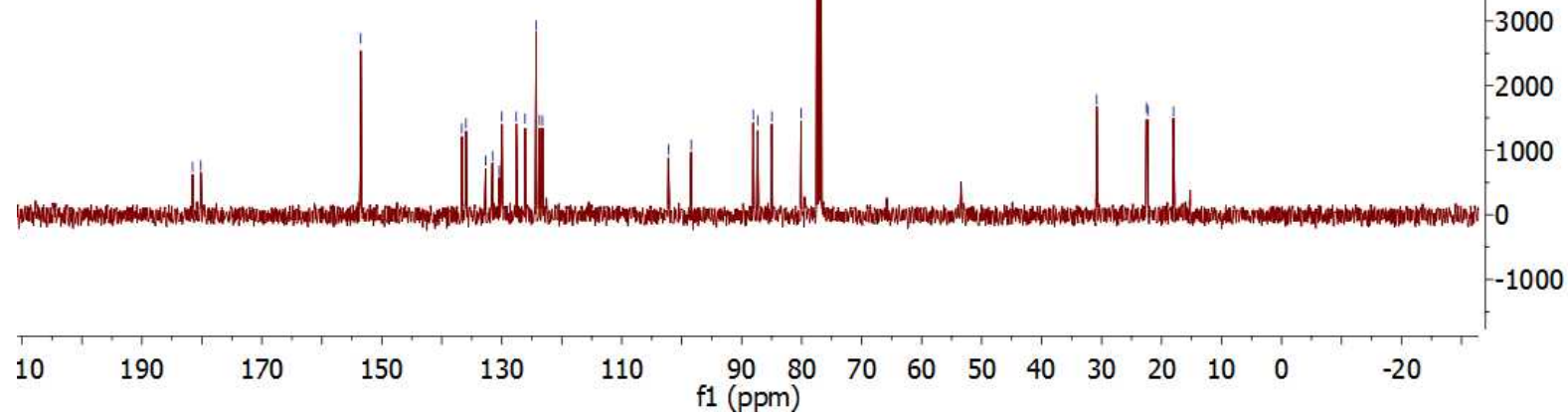


3a

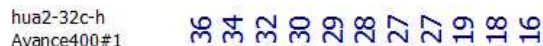

Avance400\# 1

SKSUR

Magnet Revived

minm

Revised: 21 May 15

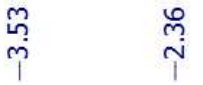

$-6000$

5500

i $\quad-5000$

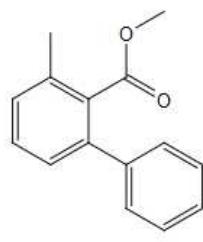

4500

4000

3500

3000

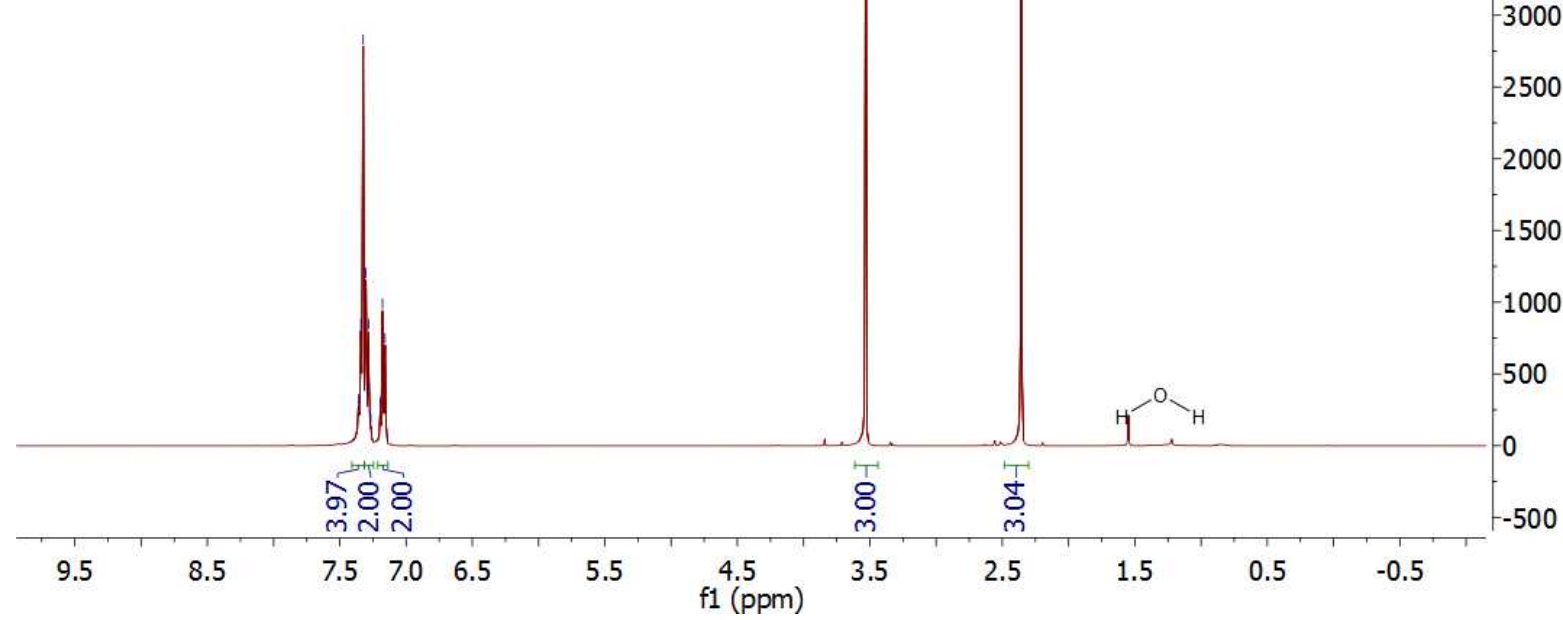

hua2-32c-c

Carbon NMR

C13_lates

Plot Paper Size: $8 \times 11$

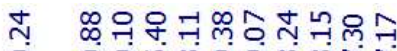

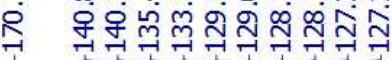

1 April 2011

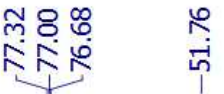

ஜே:

$-10000$

9000

8000

7000

6000

5000

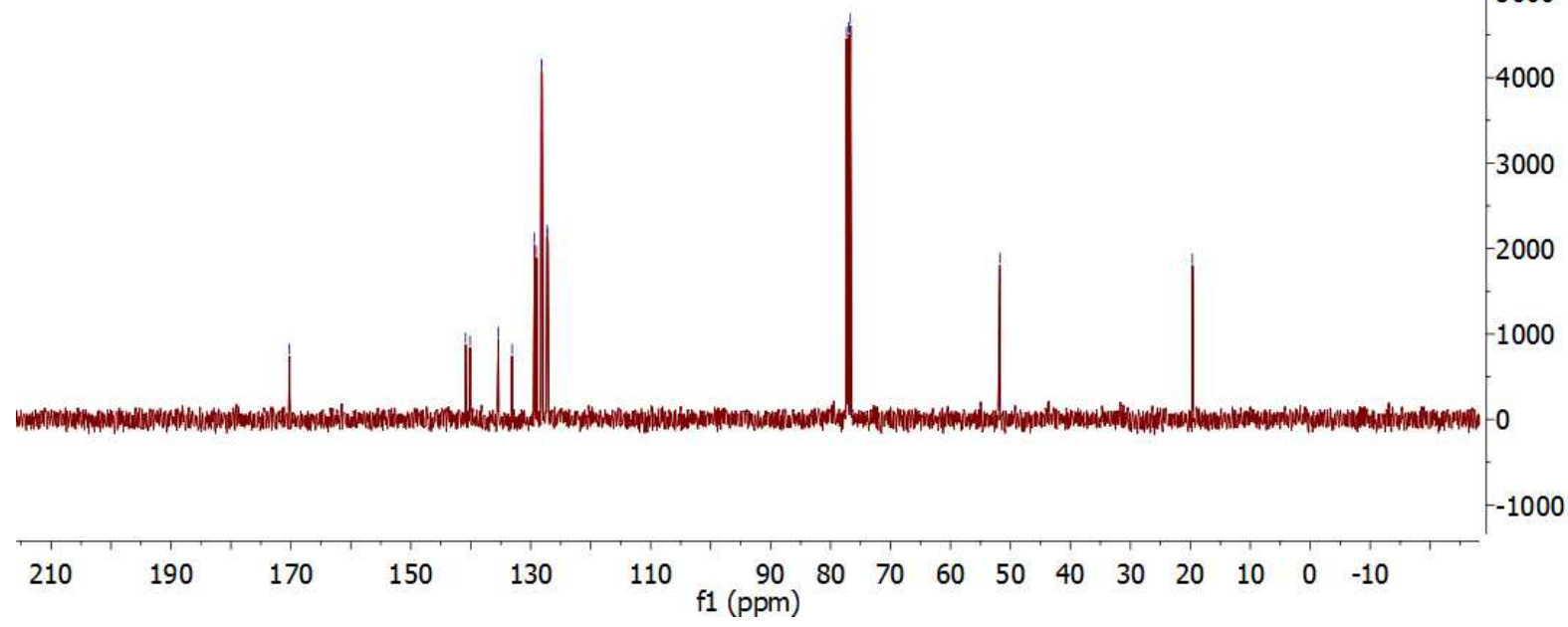




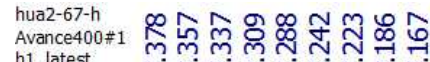

h1_latest
SKSUR

Magnet Revived

Revised: 21 May 15

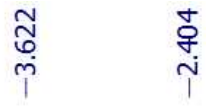

$-7000$

500

6000

5500

5000

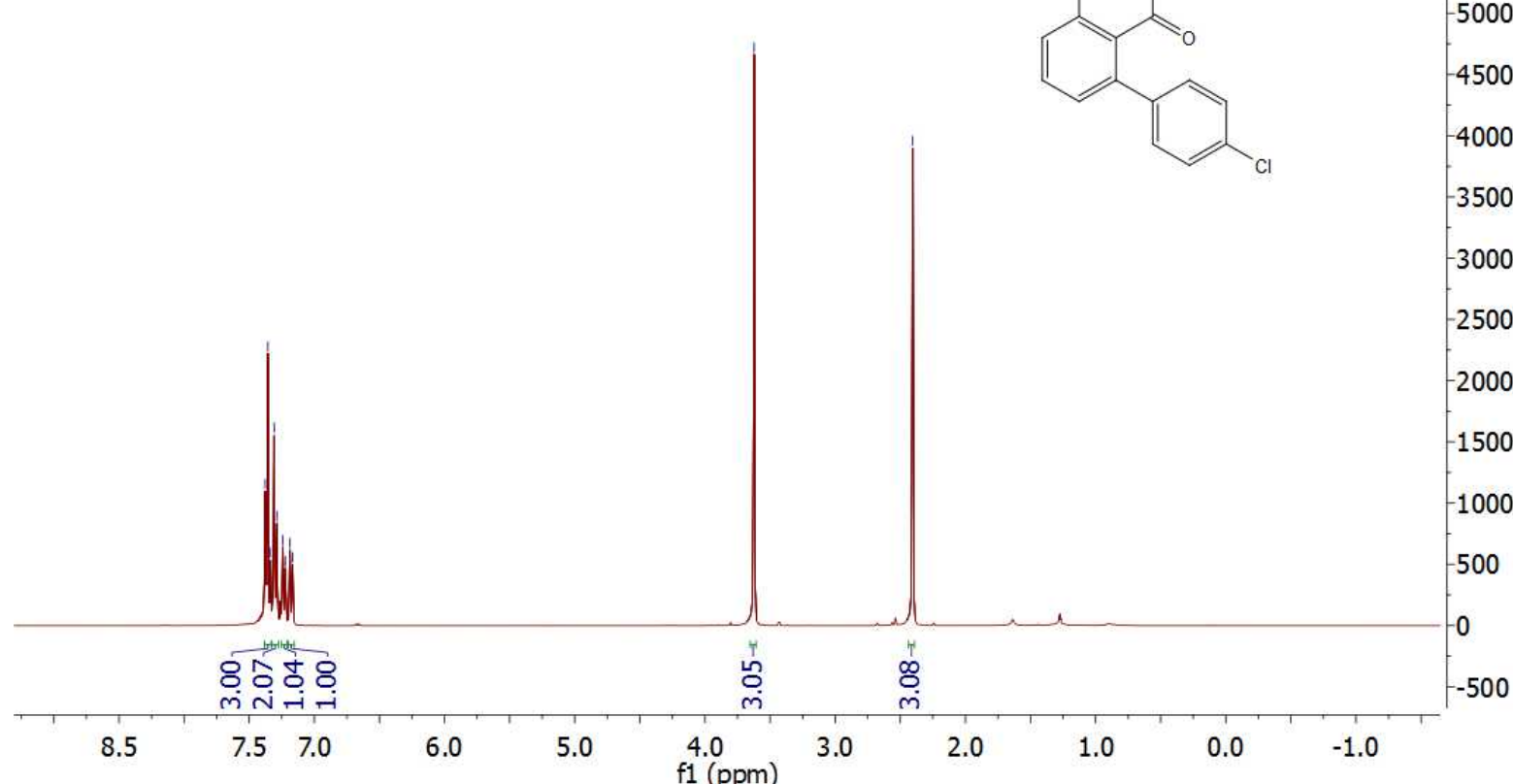

hua2-67Caron NMR Carbon NMR C13_lates

Plot Paper Size: 8x11

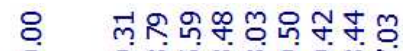

点

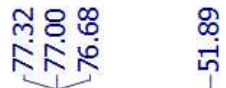

ᄂீํำ

9000

1 April 2011

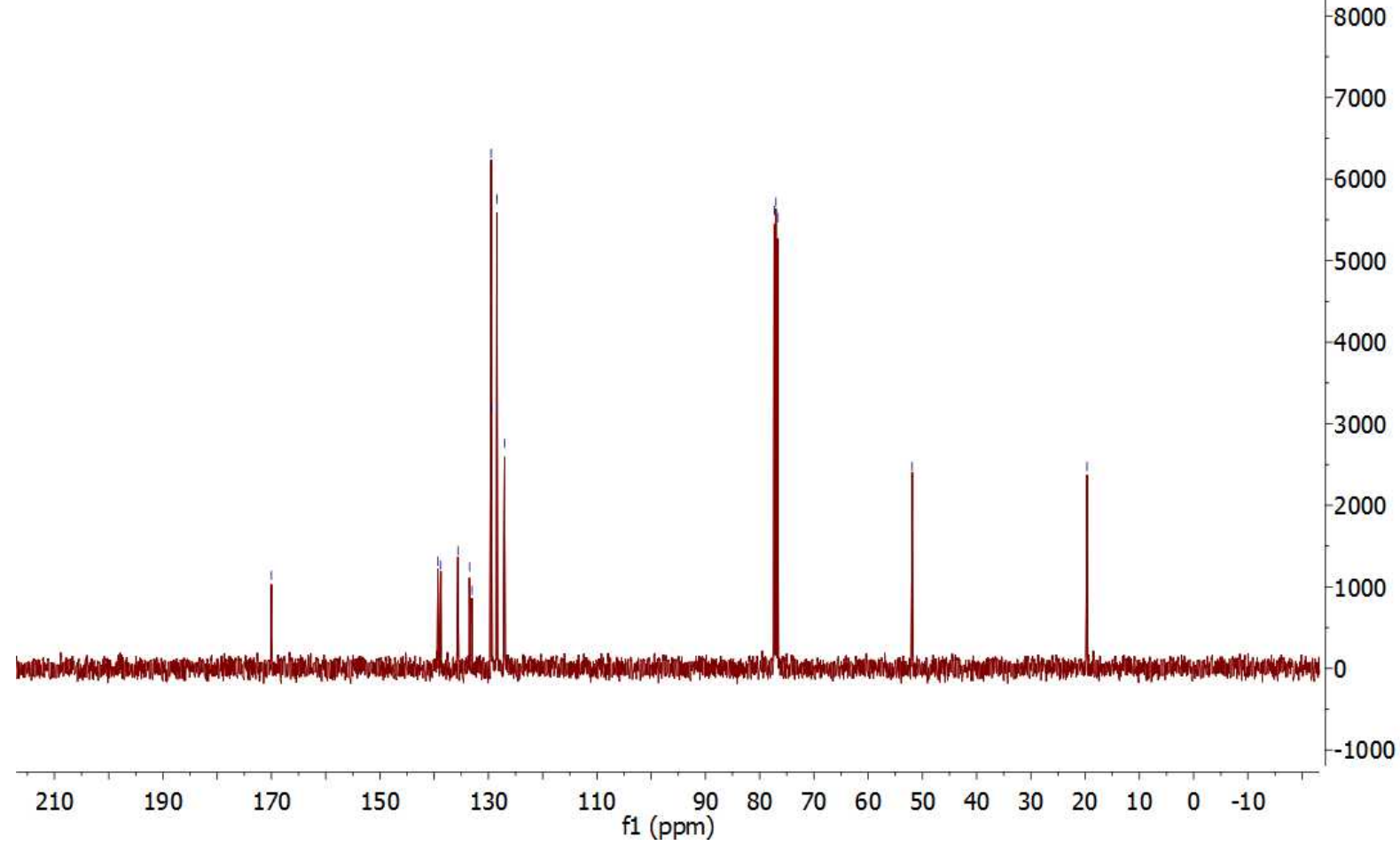




$$
3 c
$$

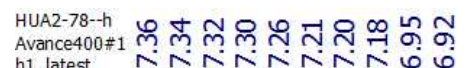

h1_latest

Magnet Revived

Revised: 21 May 15

$\dot{m} \mathfrak{m}$

mị

$\stackrel{\substack{i \\ i}}{i}$

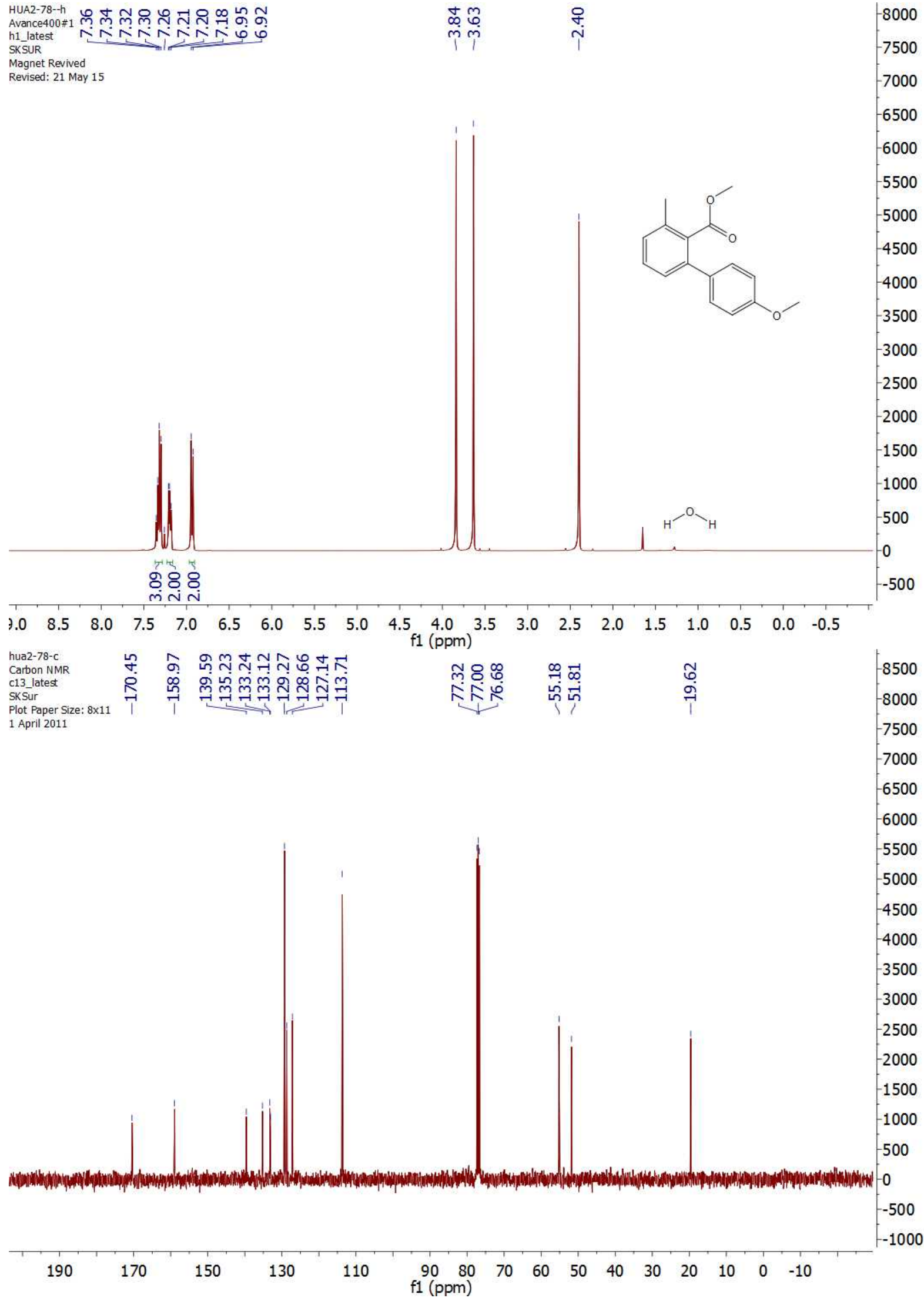

$-8000$

7500

7000

6500 


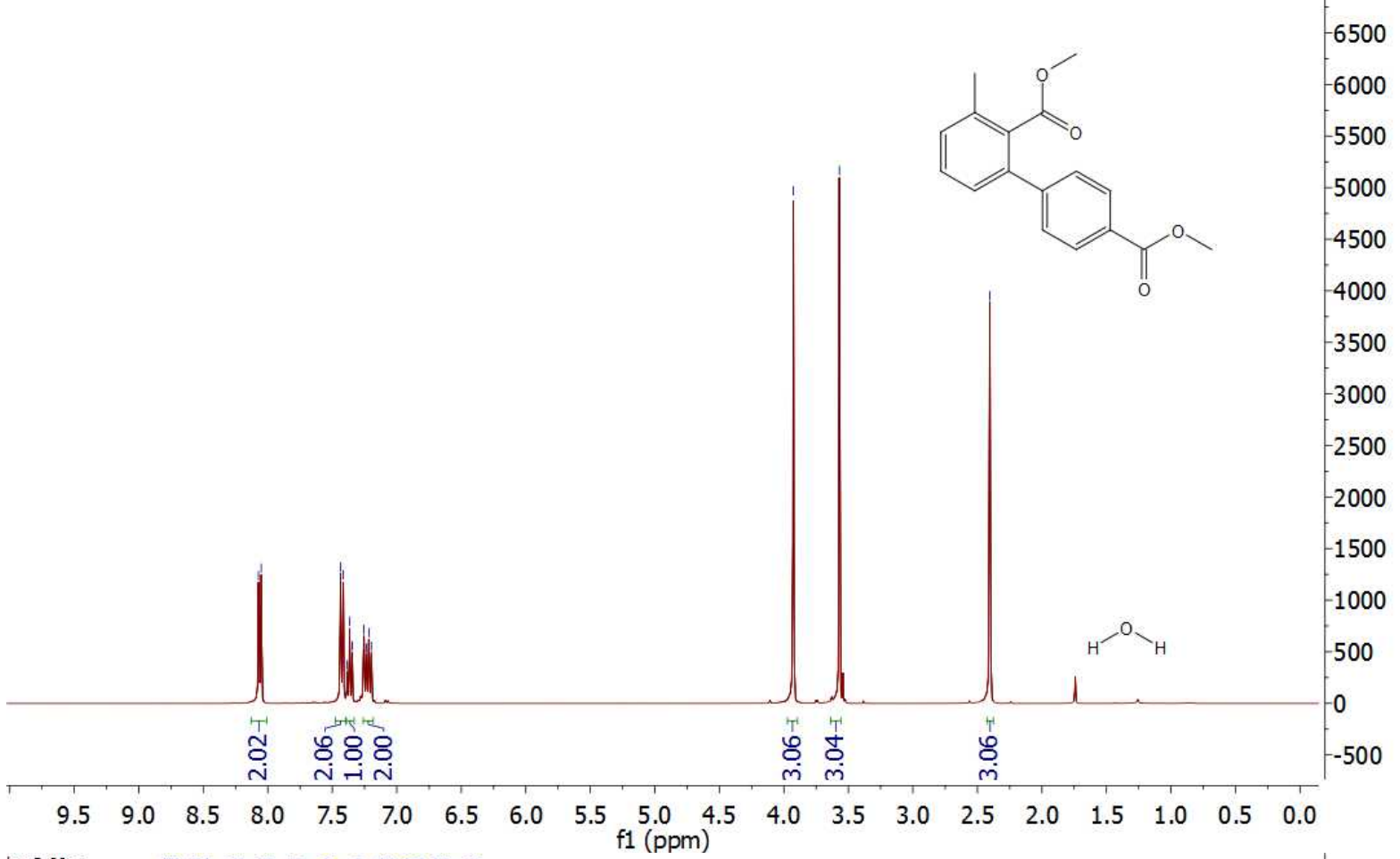

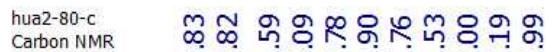

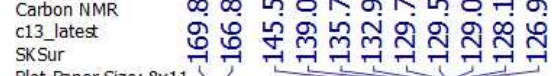

Plot Paper Siz
1 April 2011

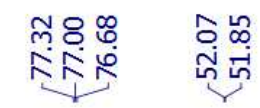

ஜํำ

$-7500$

$-7000$

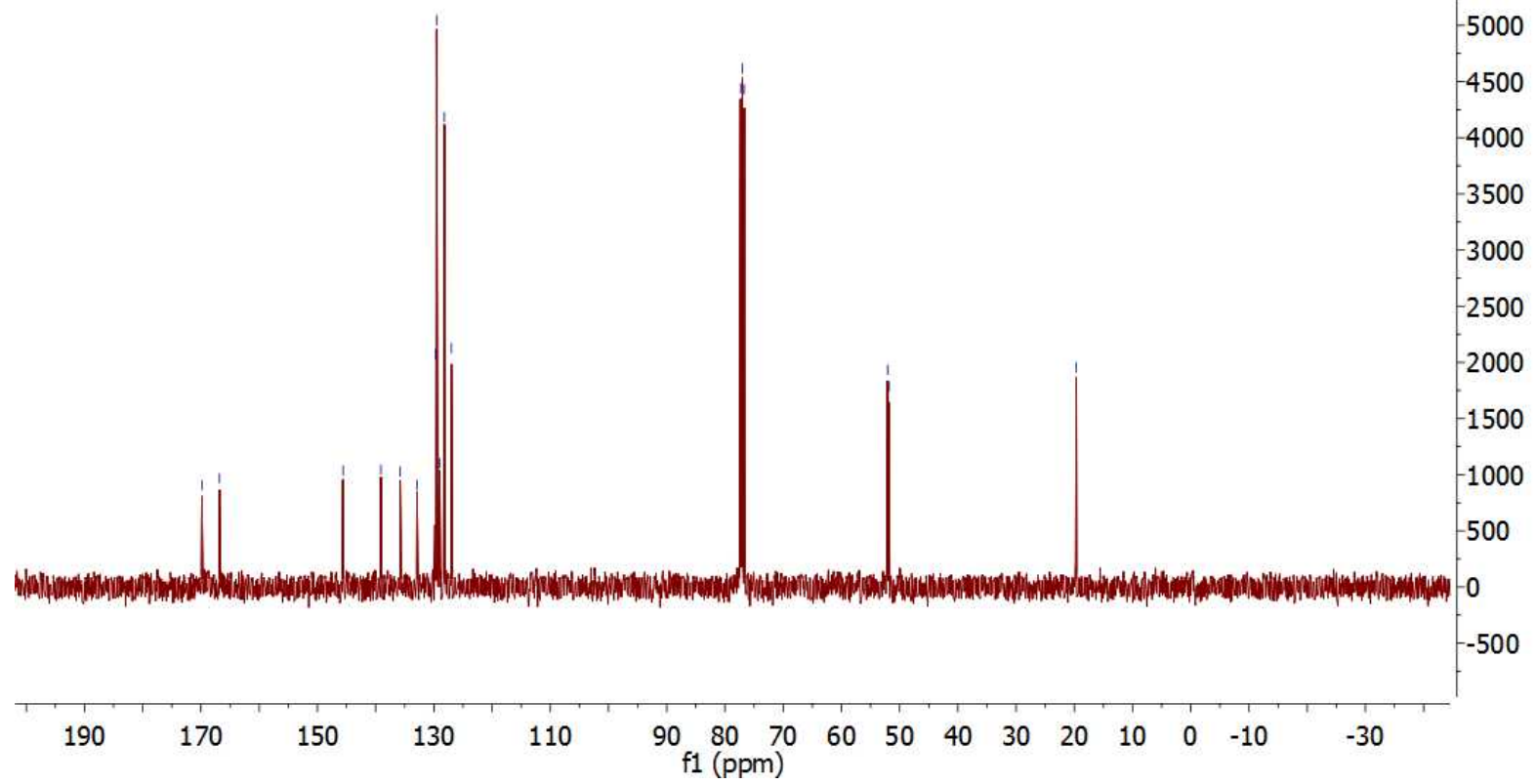


$3 e$

hua2-81-h

hua2-81-h
Avance400\#1
h1_latest

SKSUR

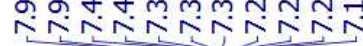

กี

$-8000$

gnet Revived

Revised: 21 May 15

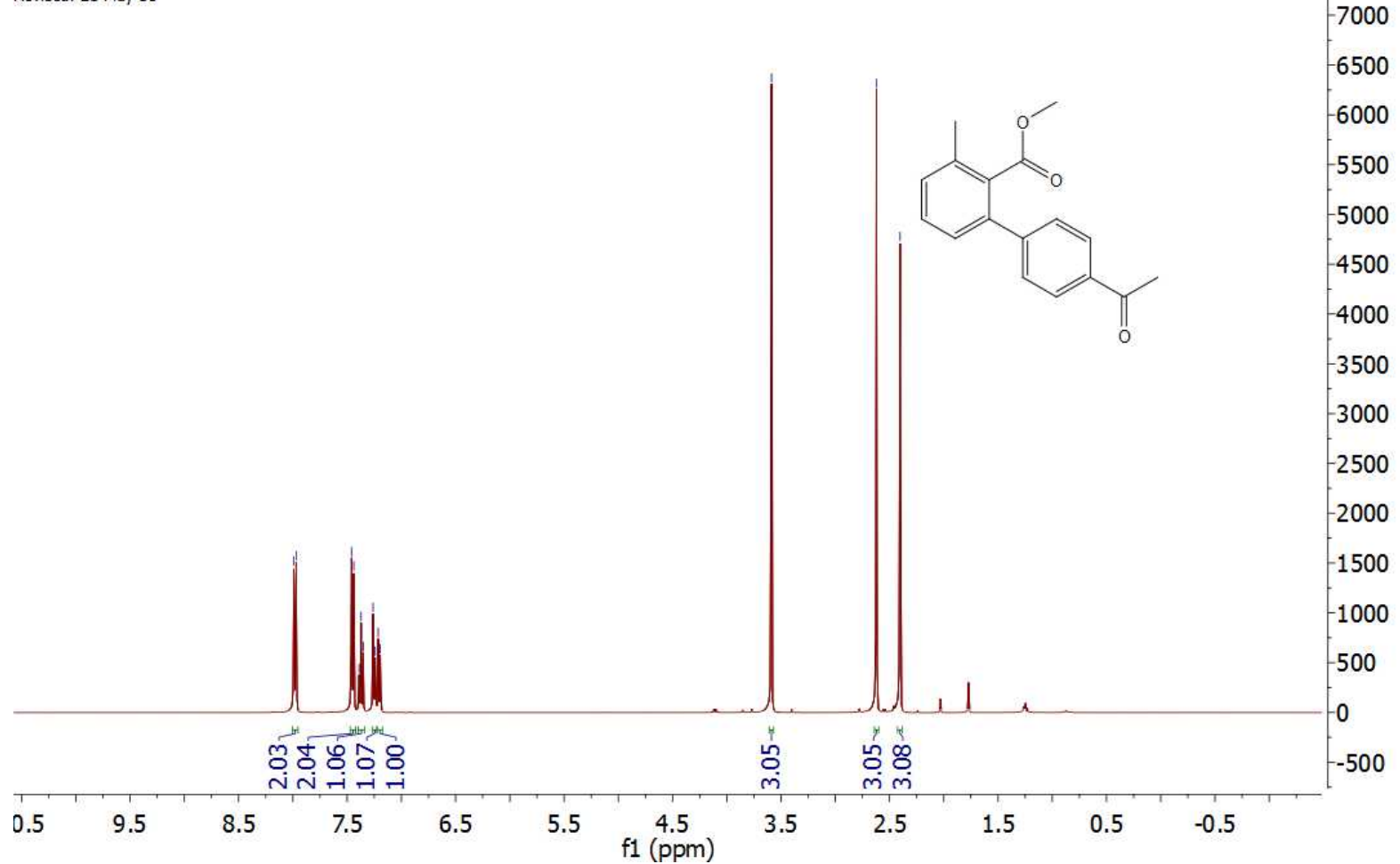

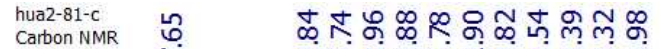

Carbon NMR latest
SKSur

Plot Paper Size: $8 \times 11$

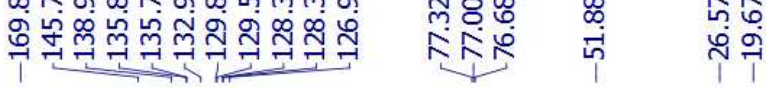

1 April 2011

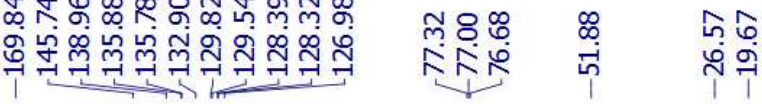

9000

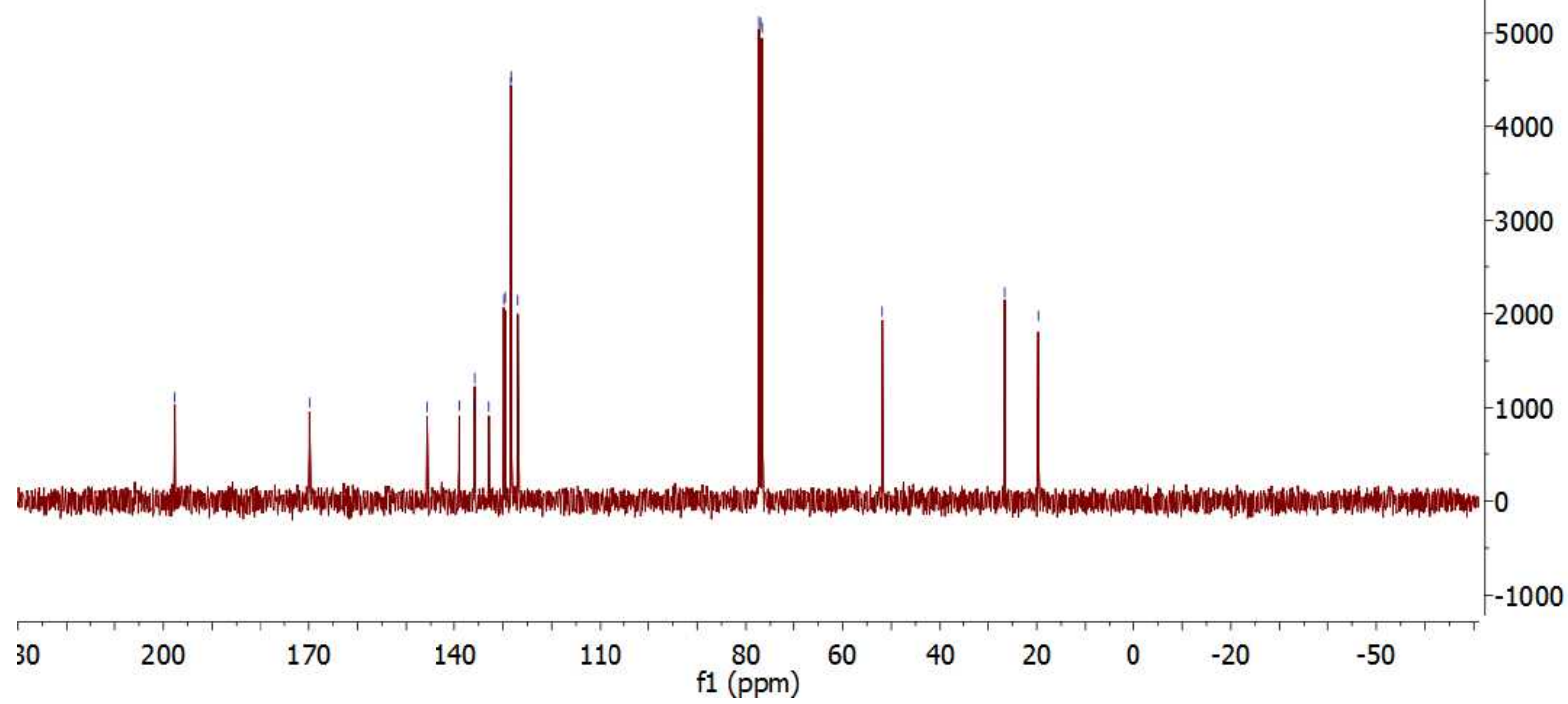



hua2-79-h
Avance400\#1
h1 latest SKSUR

Magnet Revived

Revised: 21 May 15

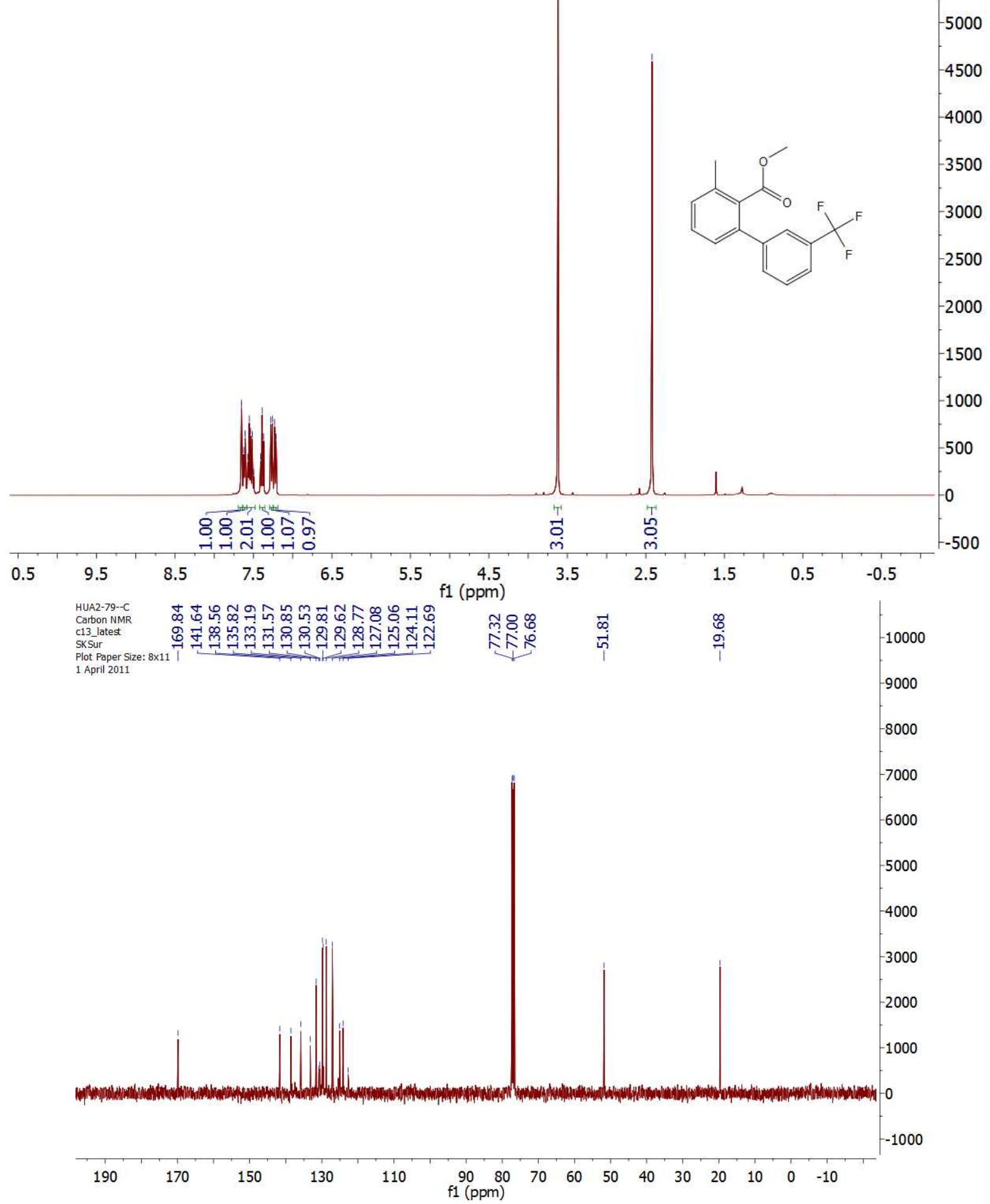


hua2-79-f

Avance 400-1

f19_latest

Plot Paper Size: $8 \times 11$

$\underset{N}{N}$

$-3200$

1 April 2011

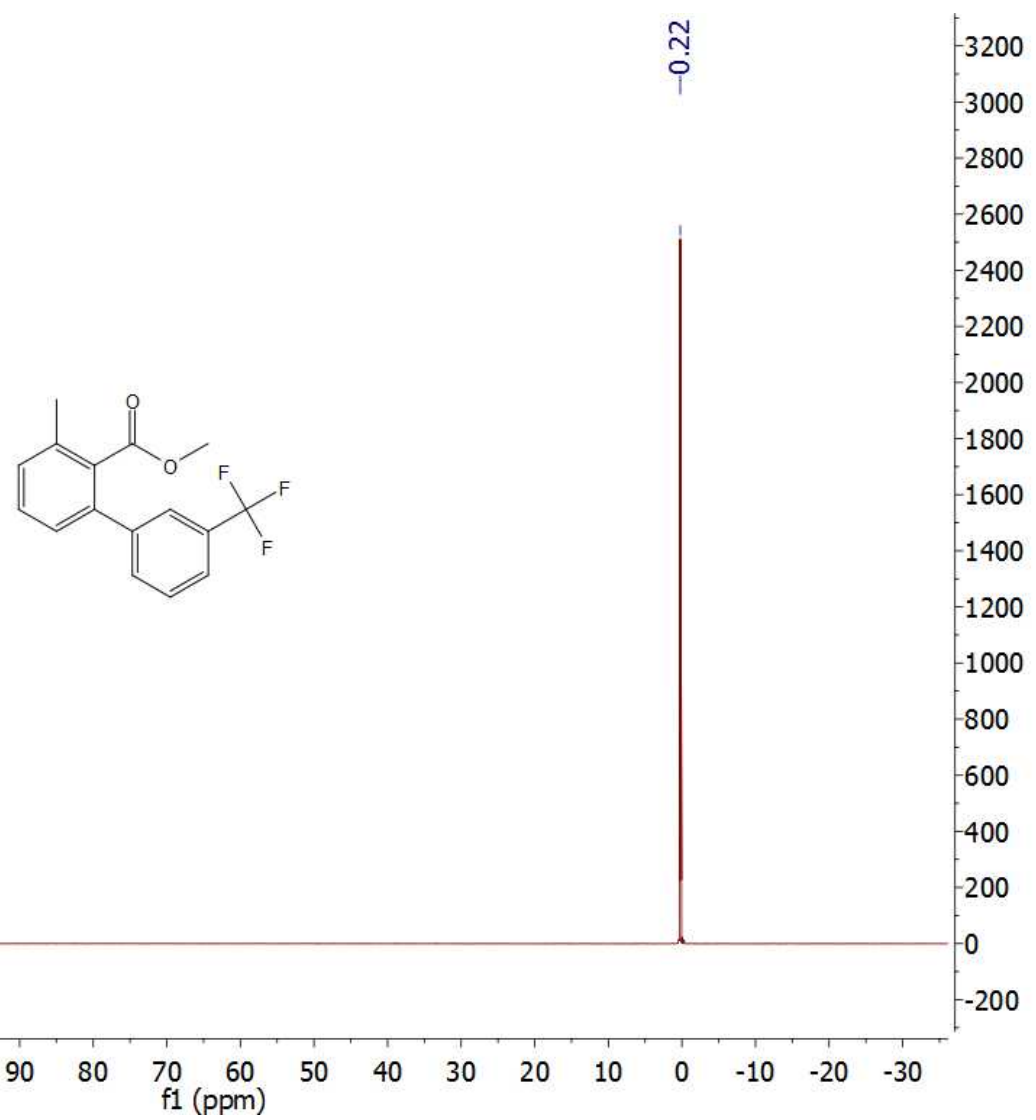


$3 g$

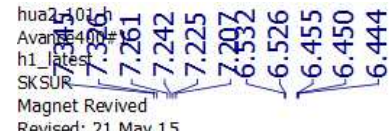

오숭

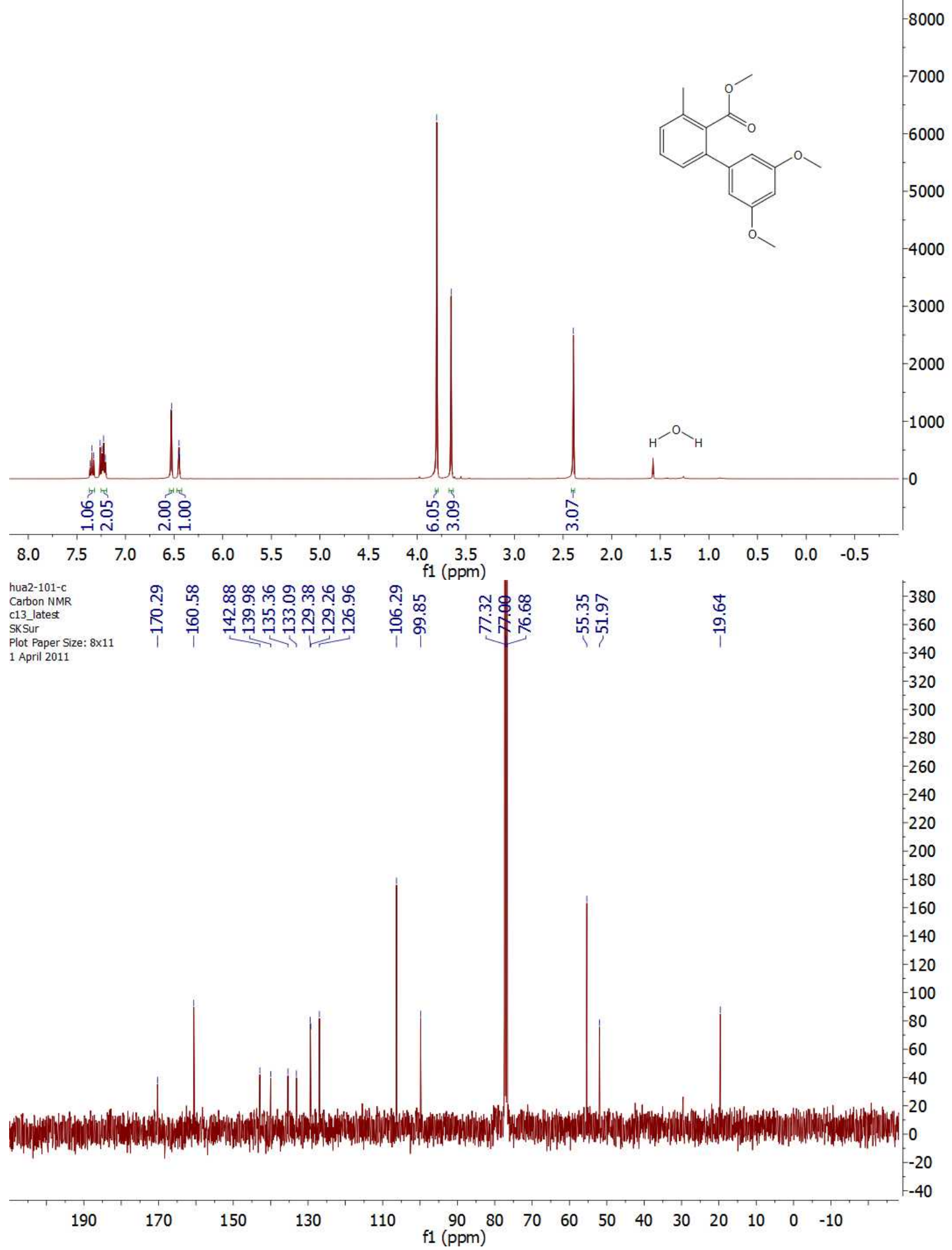


3h

hua2-94-h

Avance 400 \#1

h1_latest

Magnet Revived

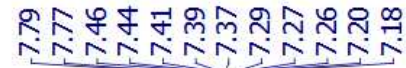

Revised: 21 May 15

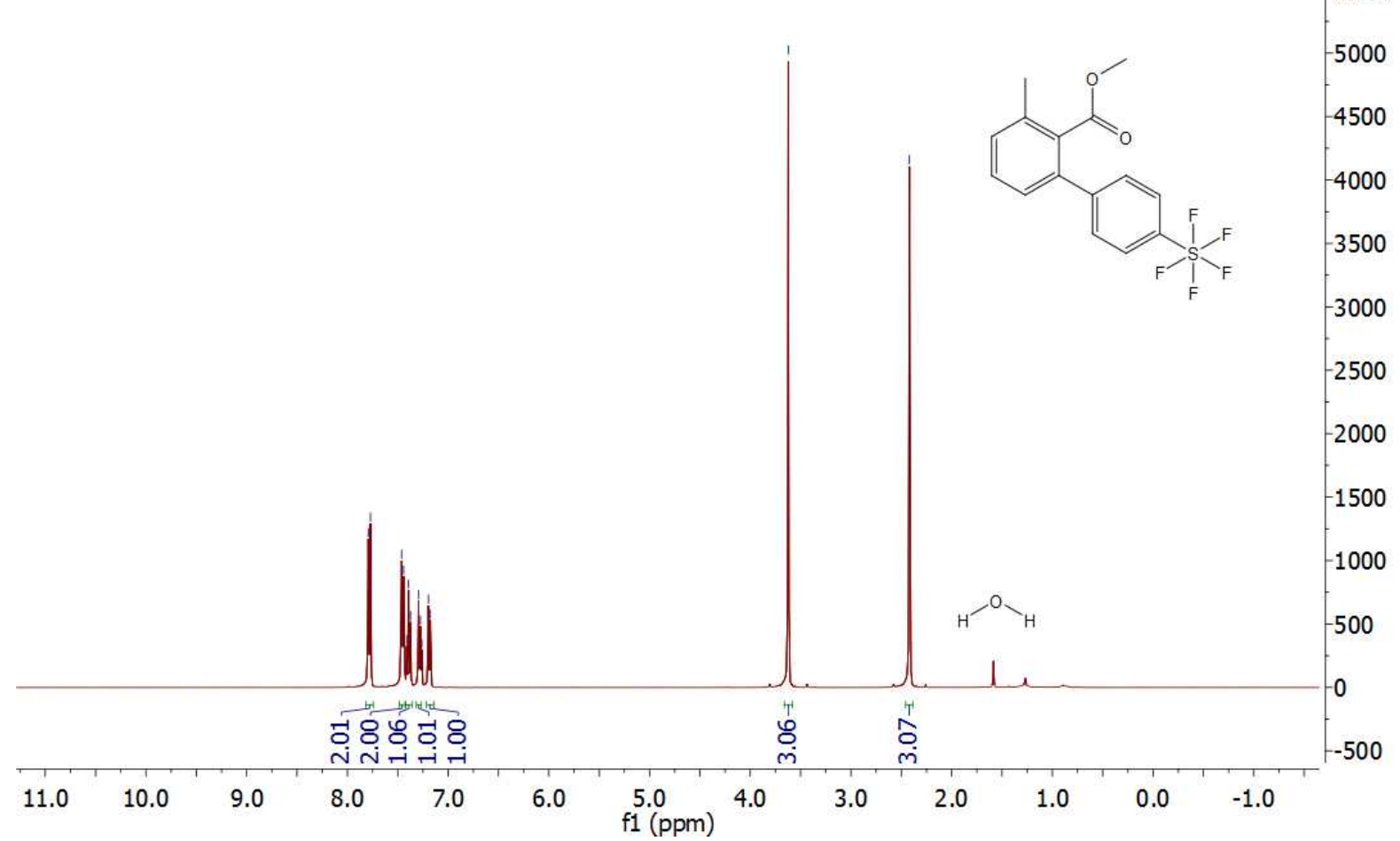

hua2-94-c Carbon NM
c13 latest C13_latest

Plot Paper Size: 8x11 1 April 2011

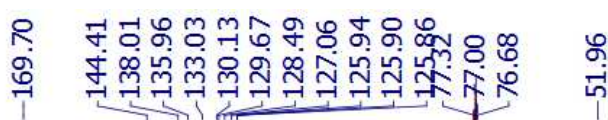

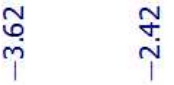

ToJu

6000

5500

000

500

000

3500

000

500

2000

500

000

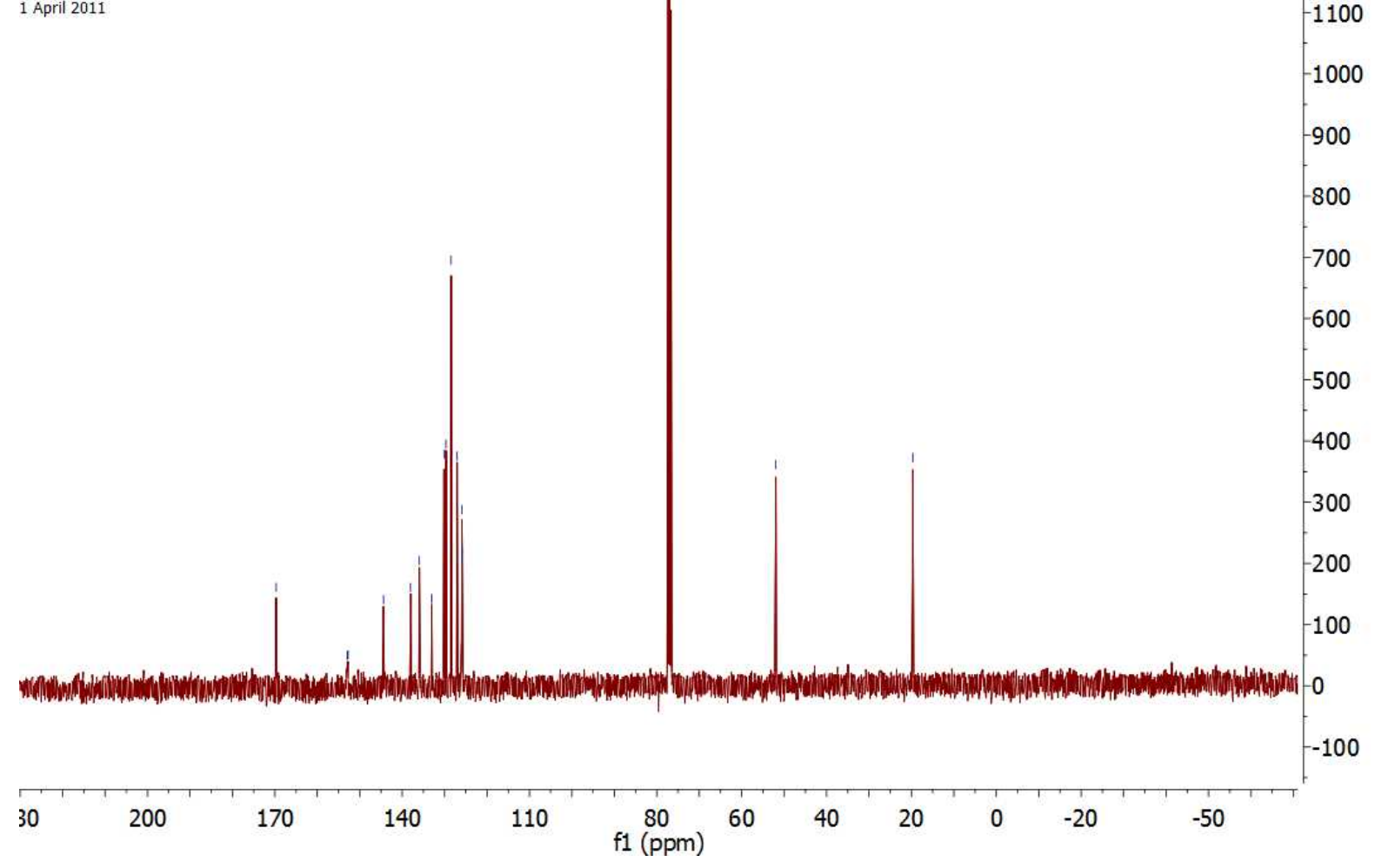




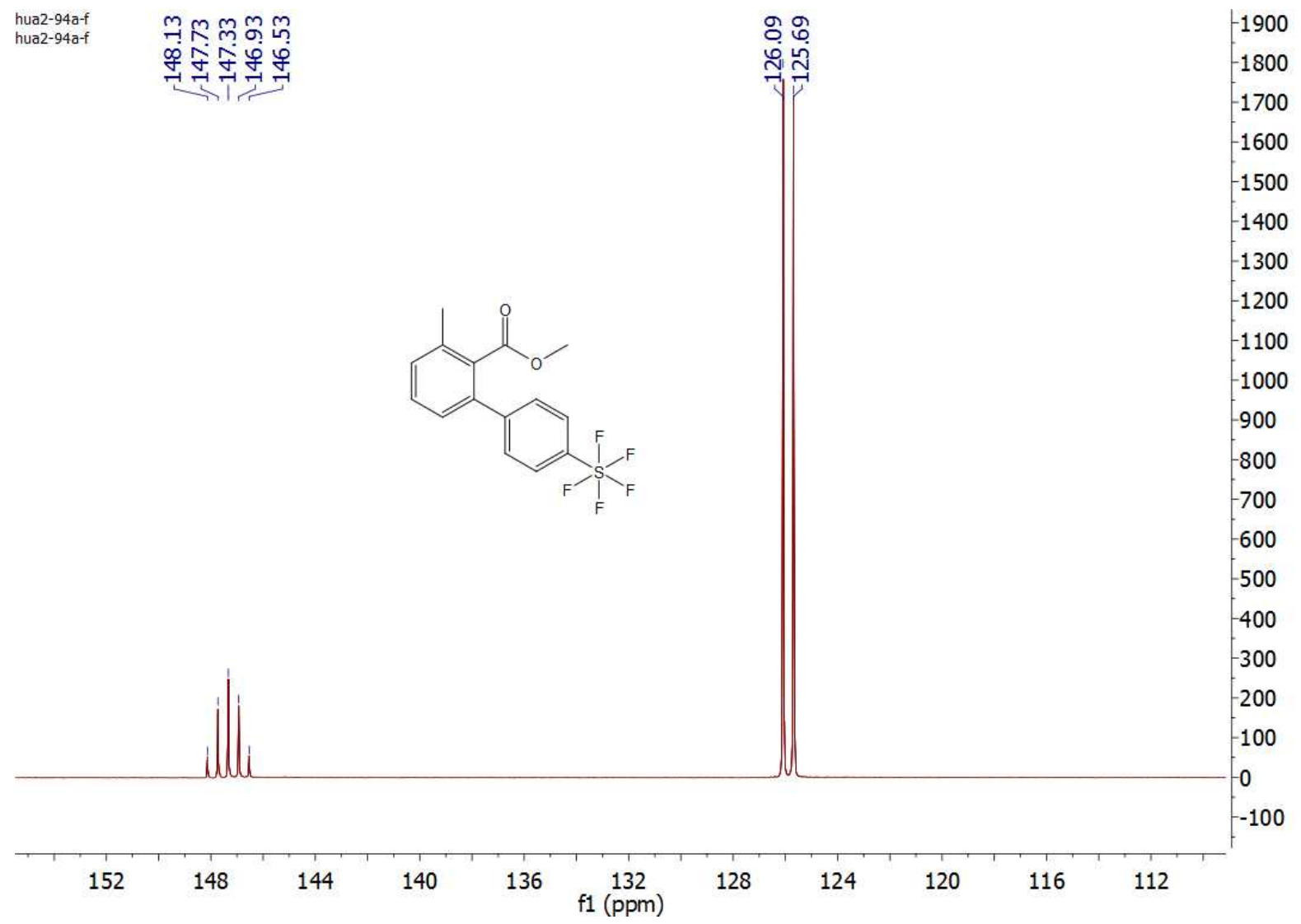


3i

hua2-103-h $\widehat{\text { Avance400\#1 }}$ m

Avance400\#1

SKSUR

Magnet Revived

Revised: 21 May 15

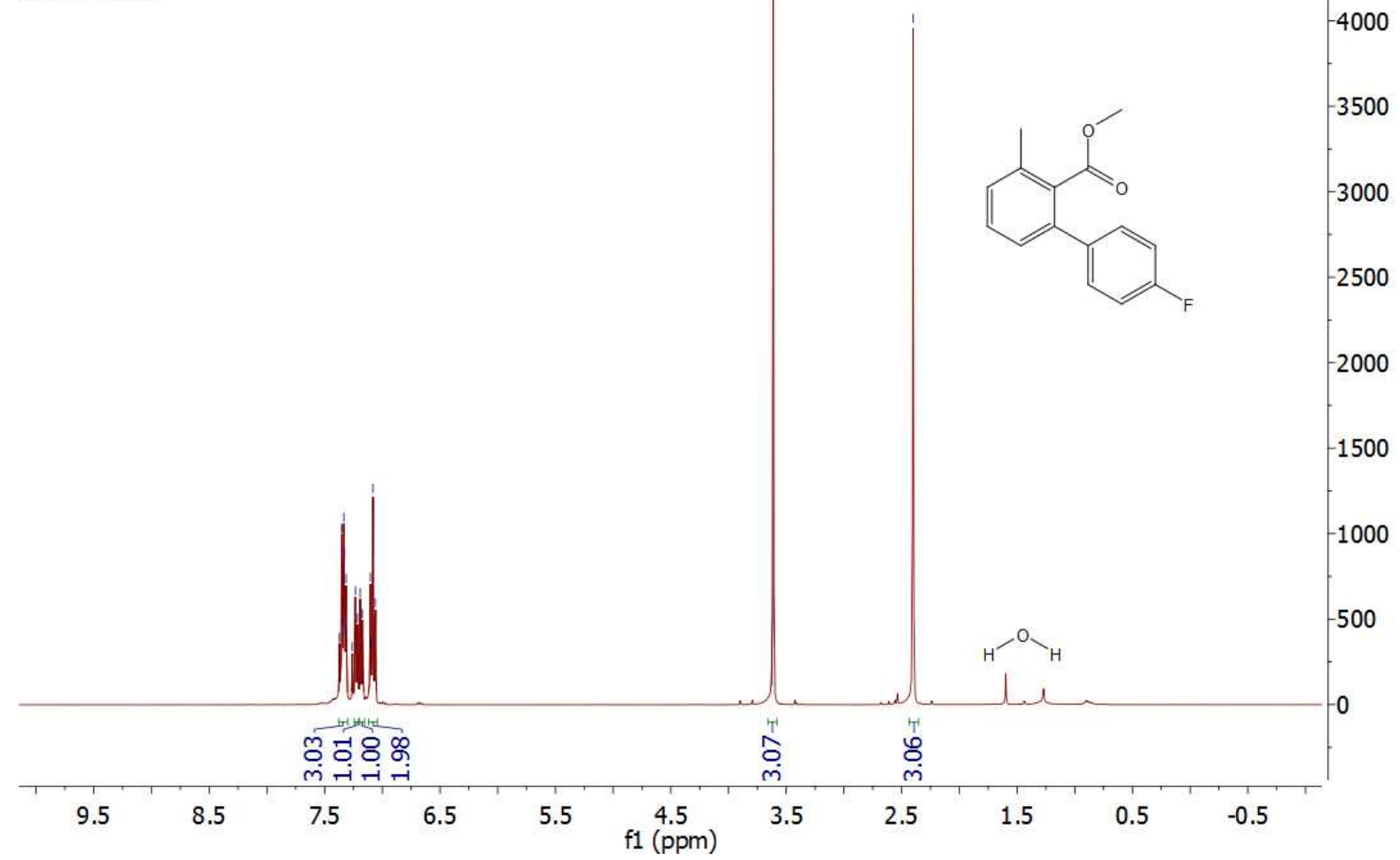

hua2-103-c

Carbon NMR

C13_latest

Plot Paper Size: $8 \times 11$

1 April 2011

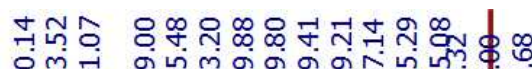

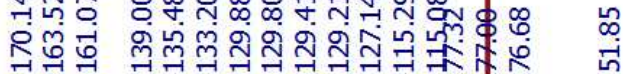

吊

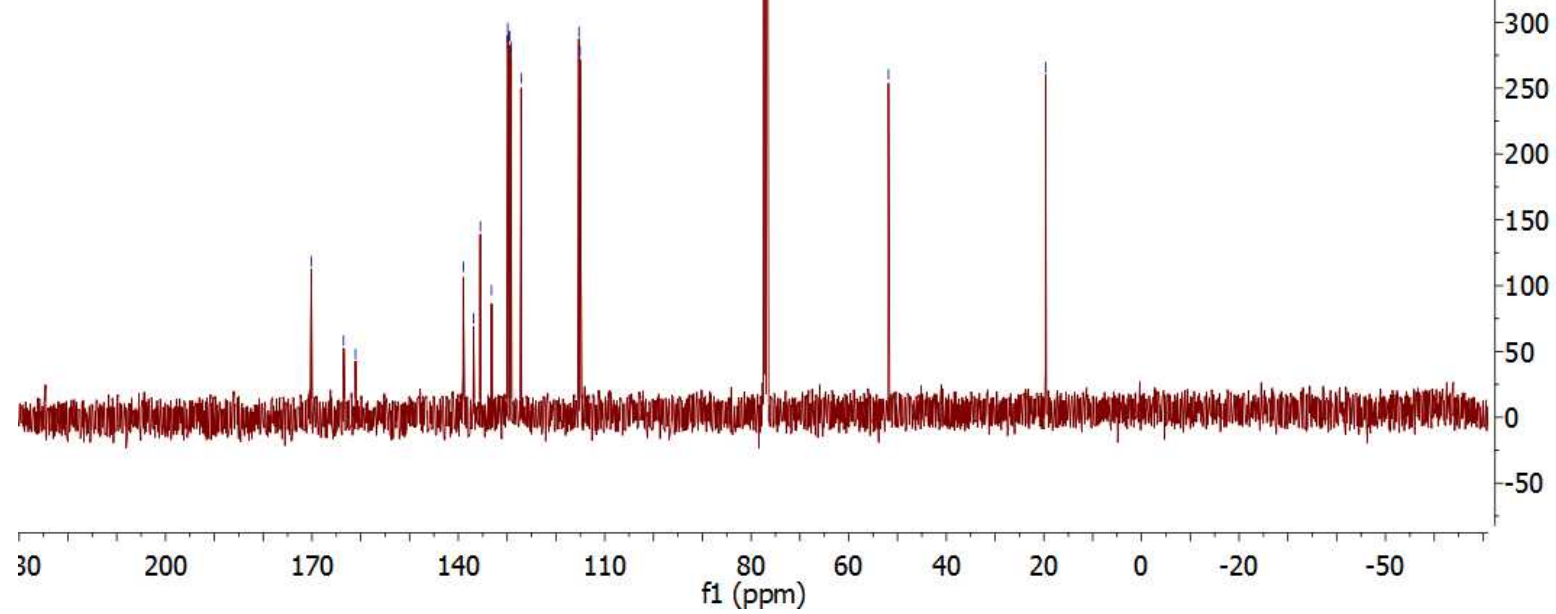




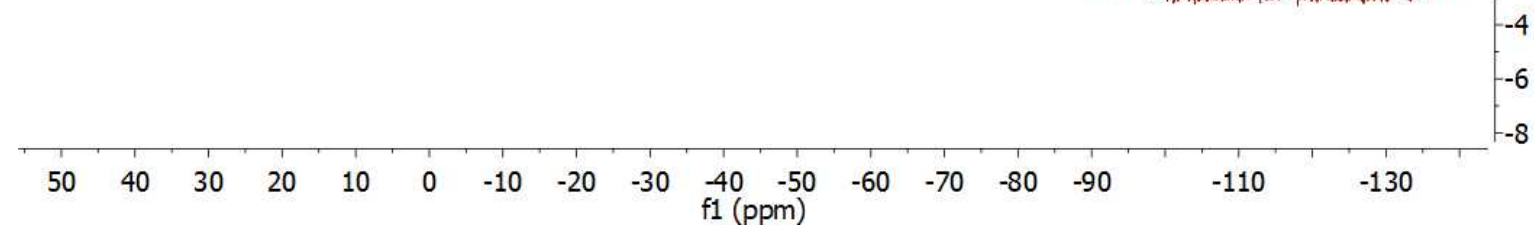


3j

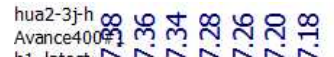

h1_latest

$\stackrel{\text { ָे }}{\text { i }}$

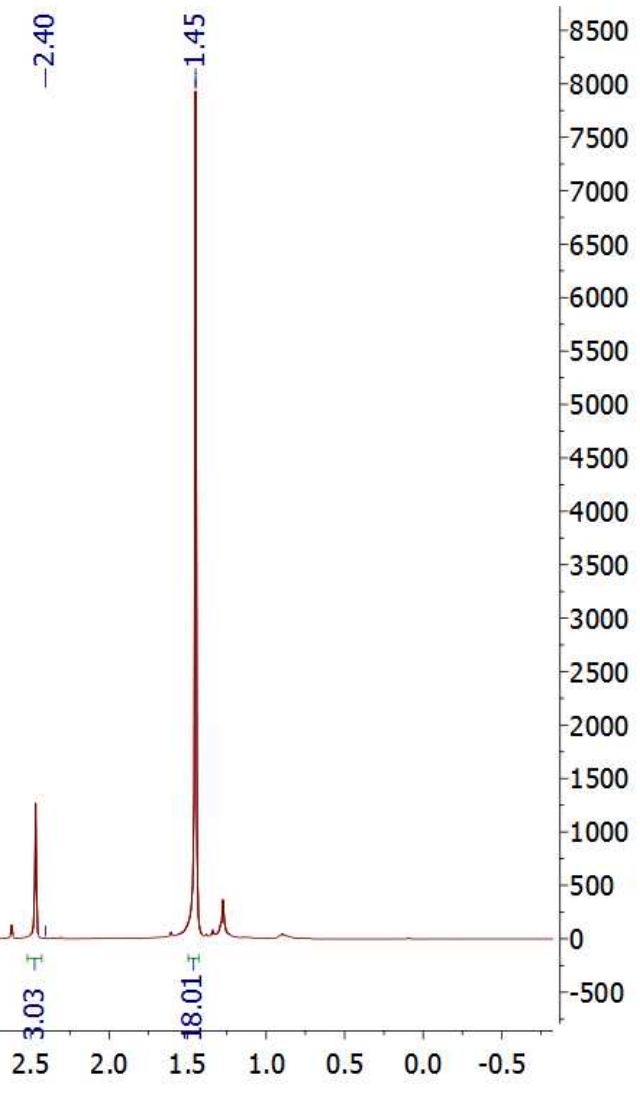

Magnet Revived
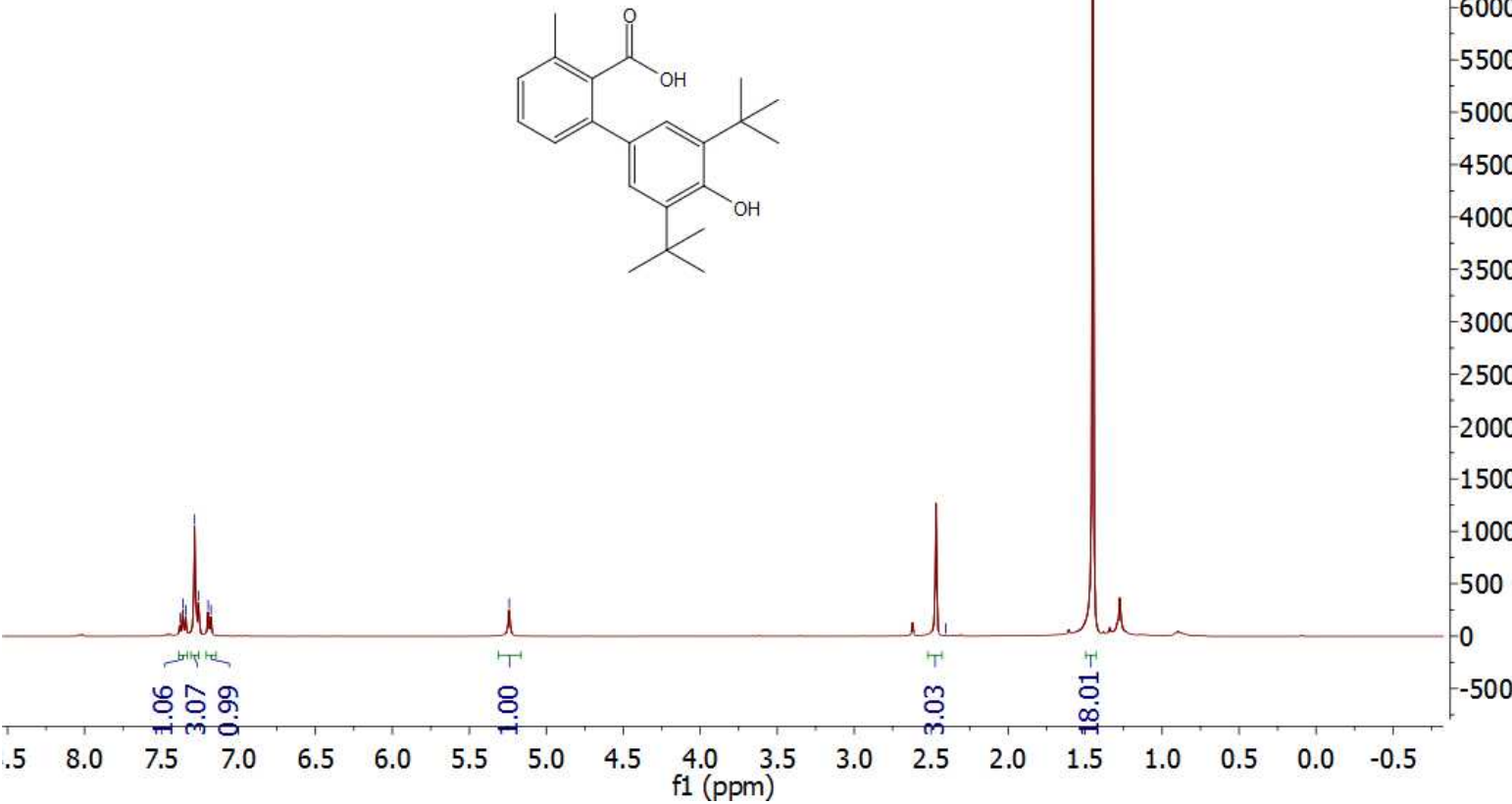

hua2-93-c
Carbon NMR

Carbon NMR m

C13_latest

Plot Paper Size: $8 \times 11$

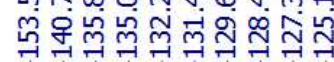

1 April 2011

f1 (ppm)

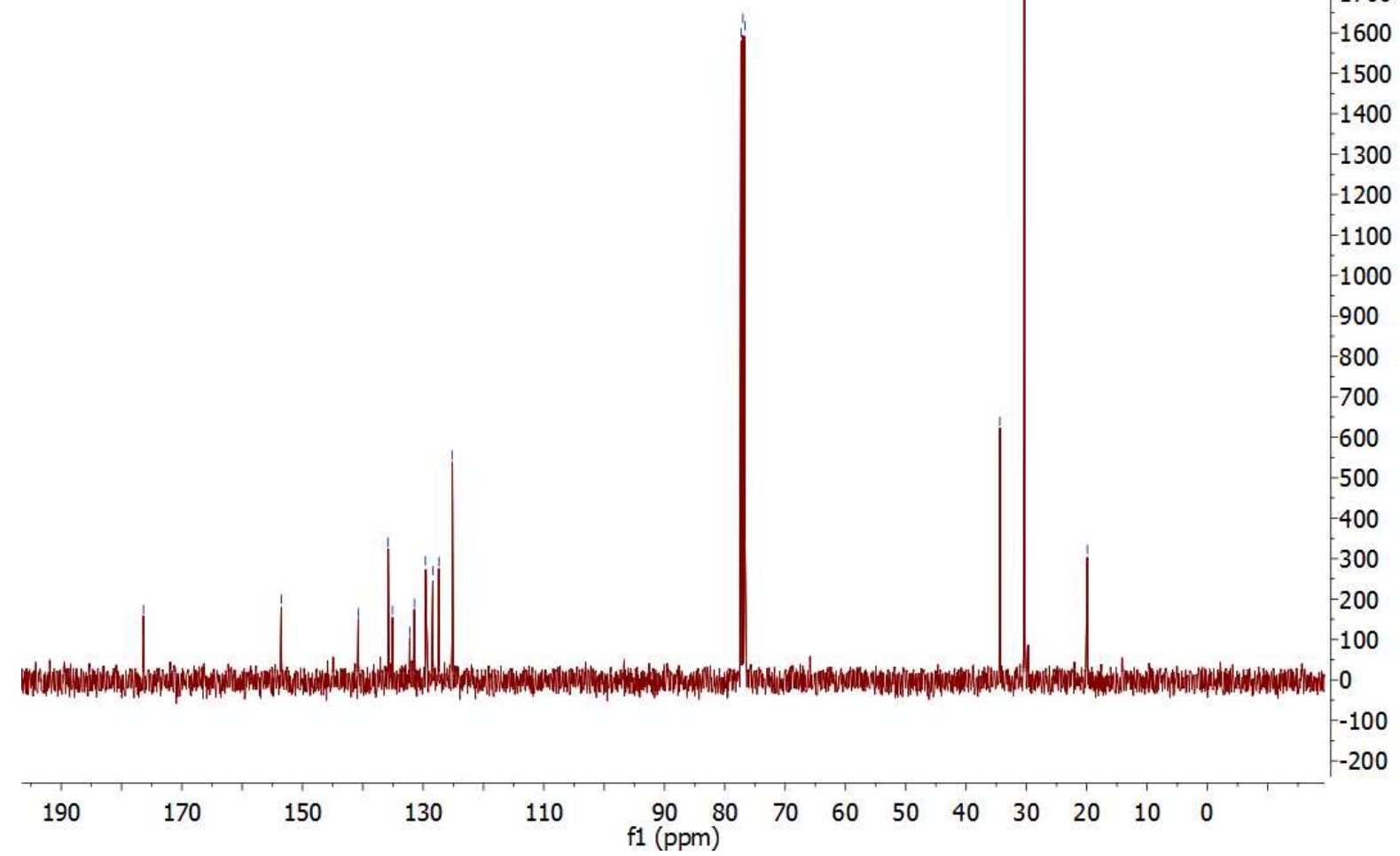


$3 k$

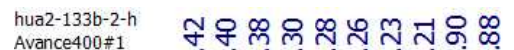

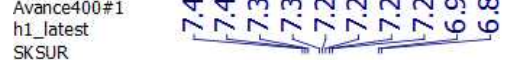

Magnet Revived

Revised: 21 May 15

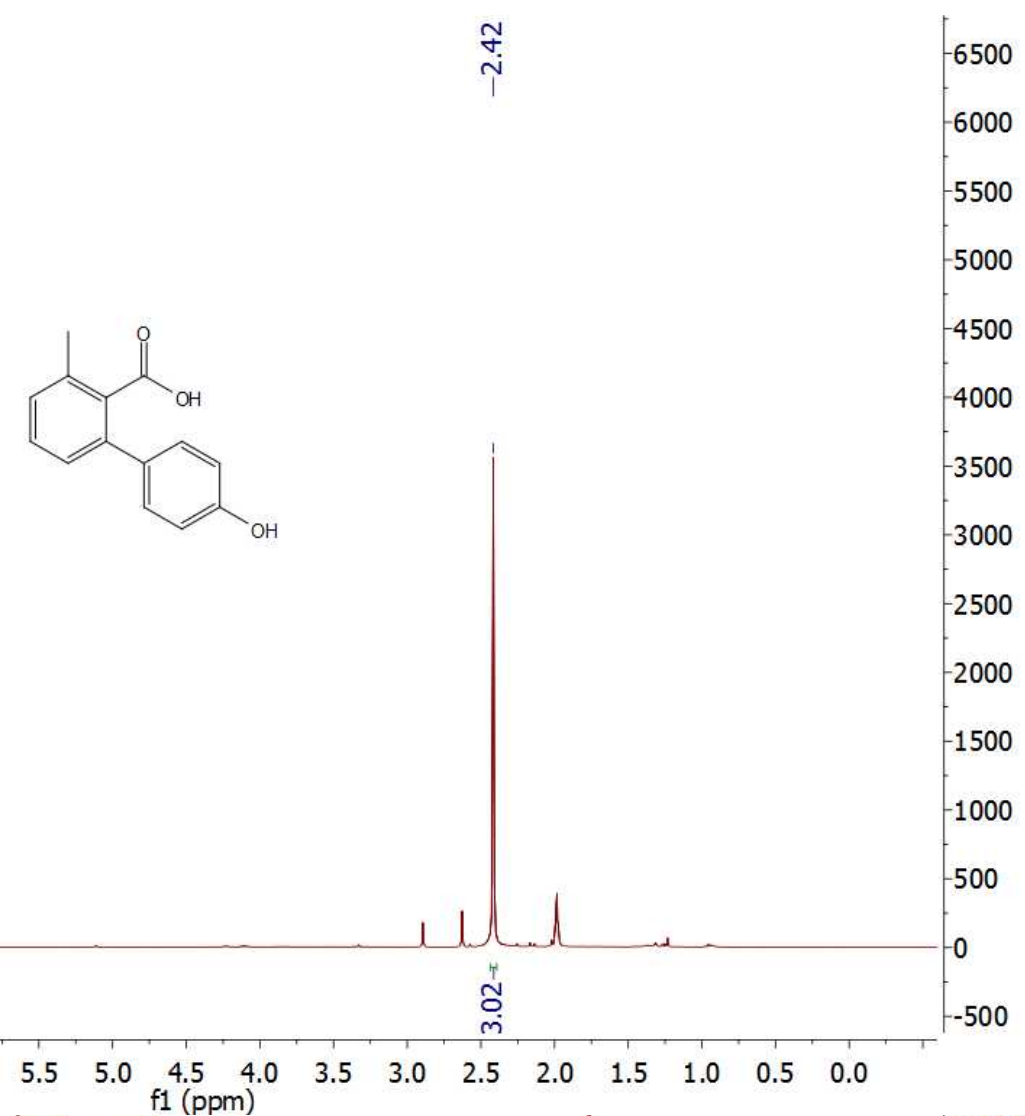

hua2-133b-2-c

Carbon NMR

C13_late

Plot Paper Size: $8 \times 11$

1 April 2011

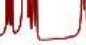

f1 (ppm)

80

$\begin{array}{llllll}2.5 & 2.0 & 1.5 & 1.0 & 0.5 & 0.0\end{array}$

00

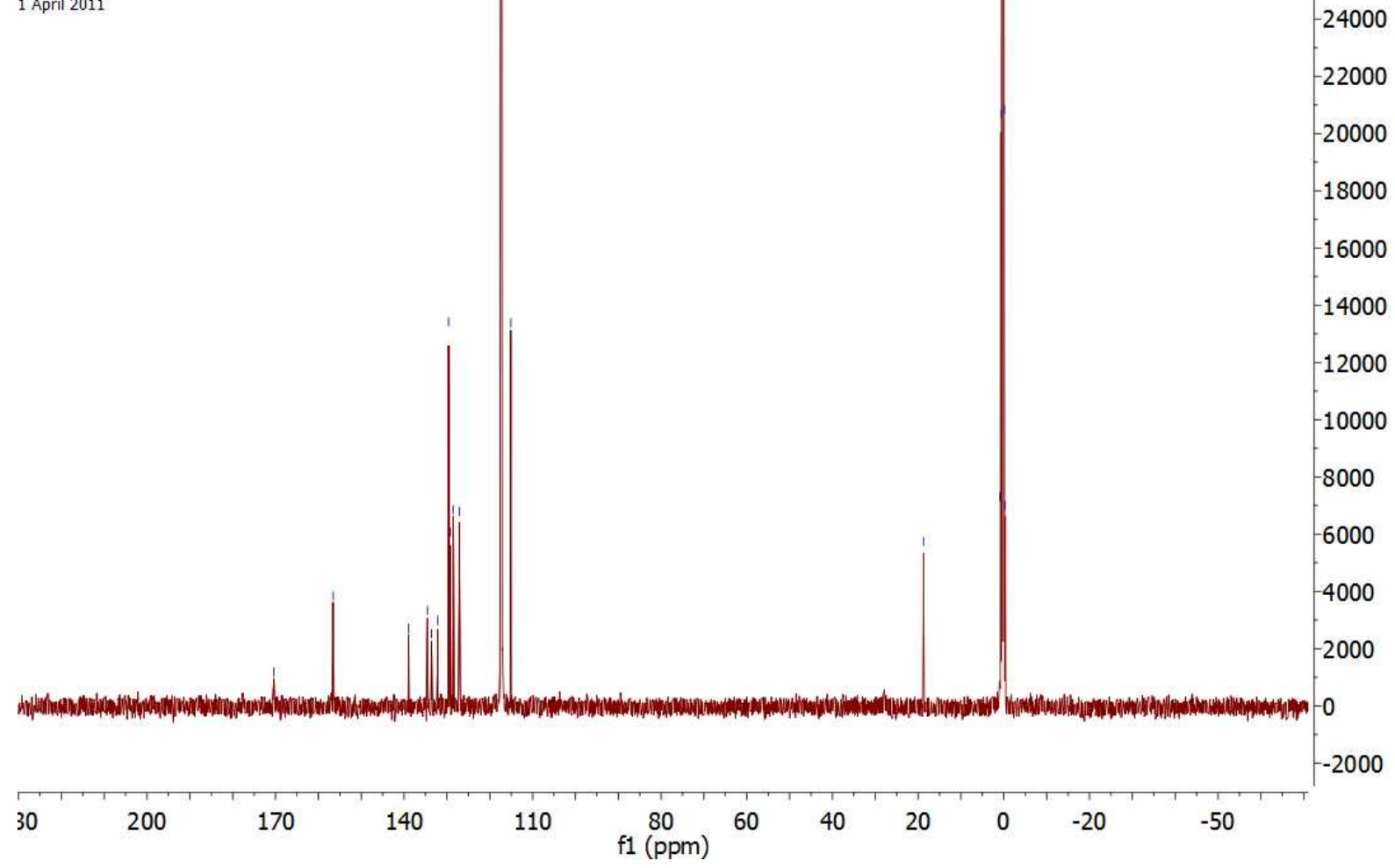


为20645

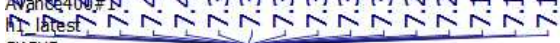
SKSUR

Magnet Revived

Revised: 21 May 15

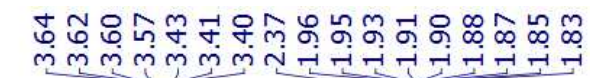
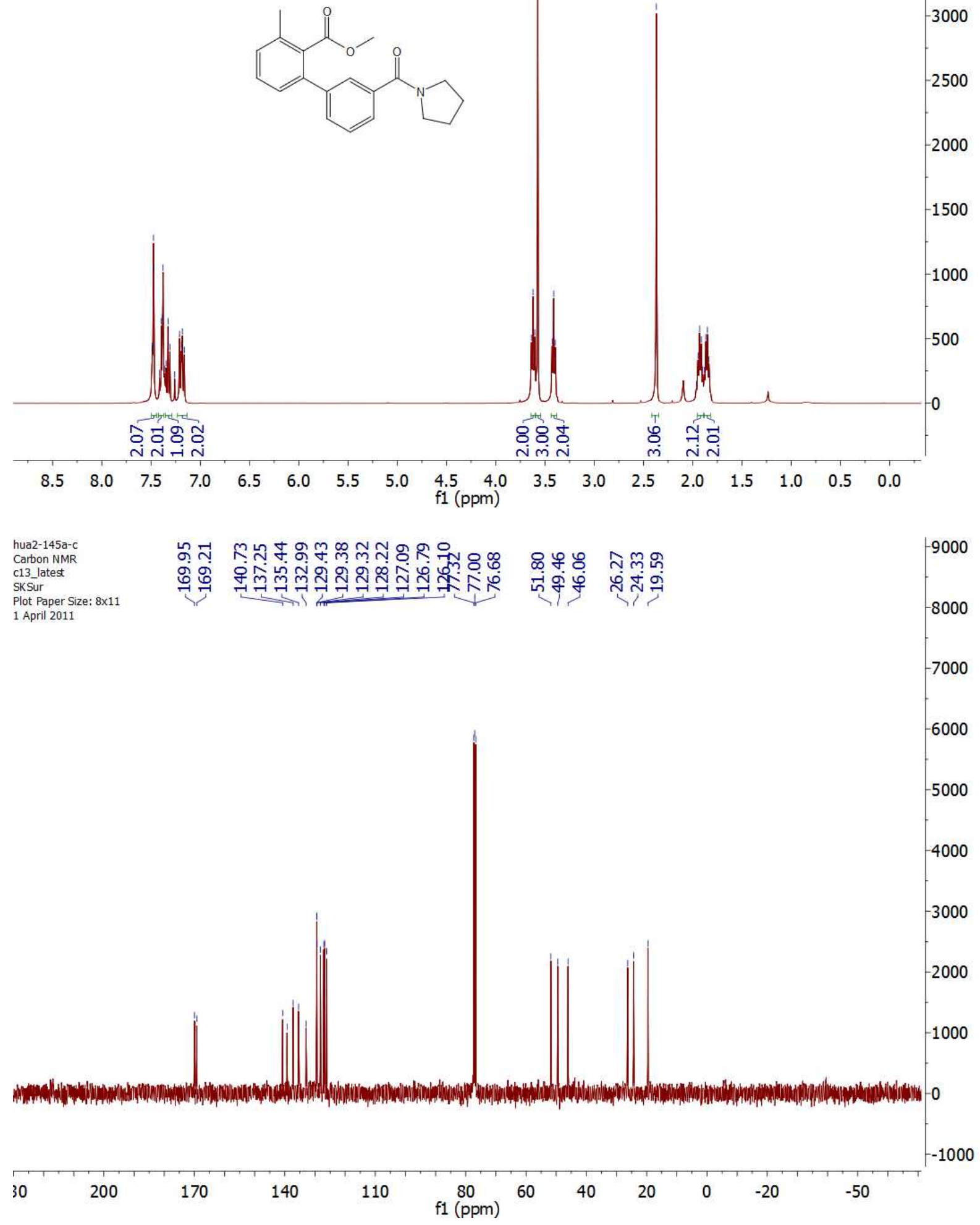
hua2-154a-2th ન્

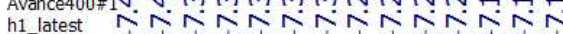

SKSUR

Magnet Revived

Revised: 21 May 15

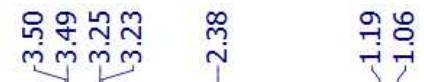

$-3000$

Rench 21 May 15
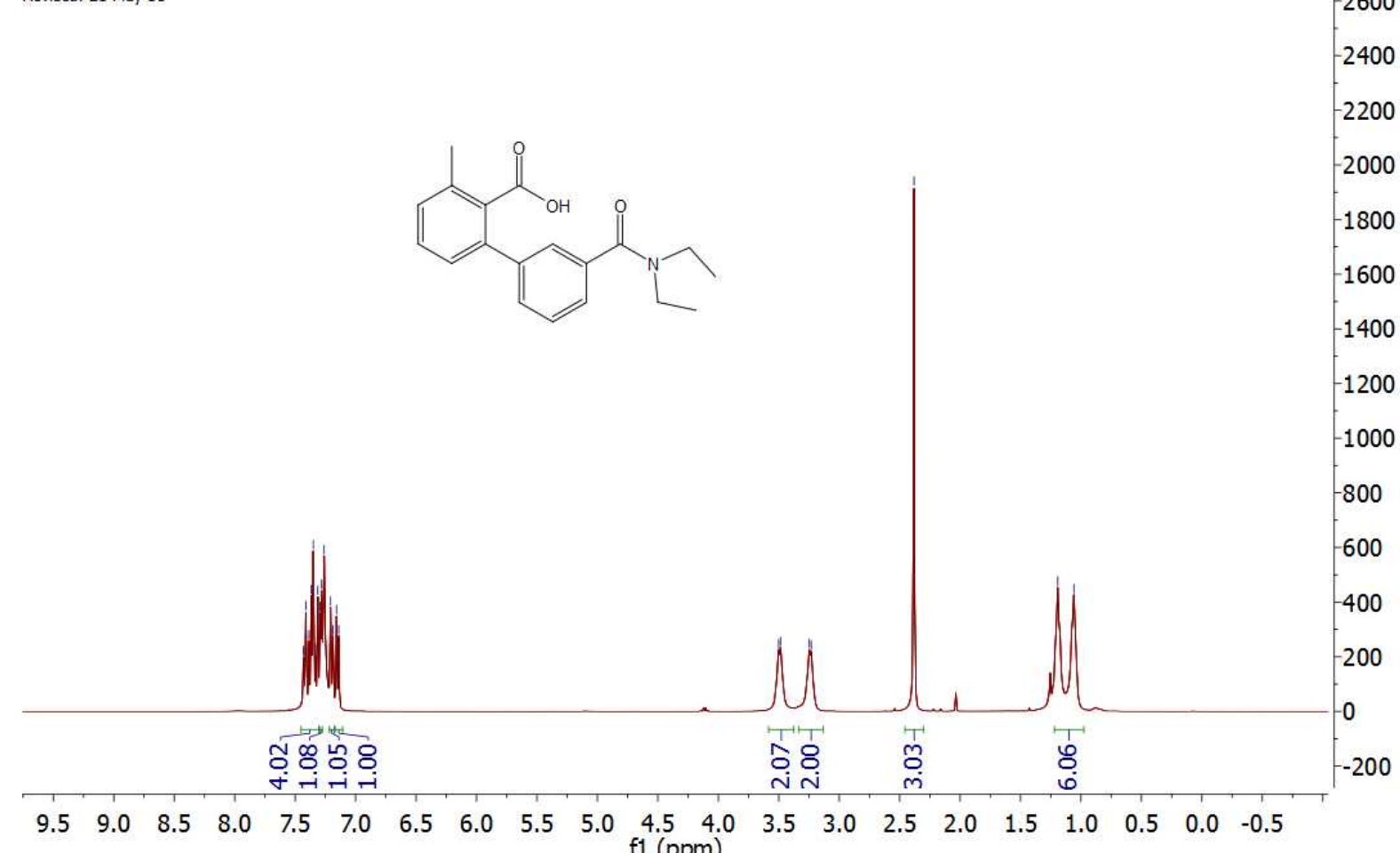

-2800
-2600
-2400

hua2-154a-2-c

hua2-154a-2-c
Carbon NMR

C13_latest

Plot Paper Size: $8 \times 11$

1 April 2011

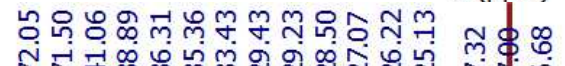

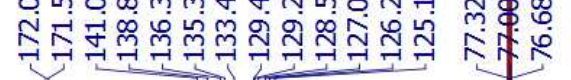

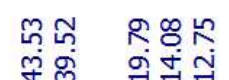

5 S4UUU

$-32000$

$-30000$

$-28000$

$-26000$

$-24000$

$-22000$

$-20000$

$-18000$

$-16000$

$-14000$

$-12000$

$-10000$

$-8000$

6000

4000

$-2000$

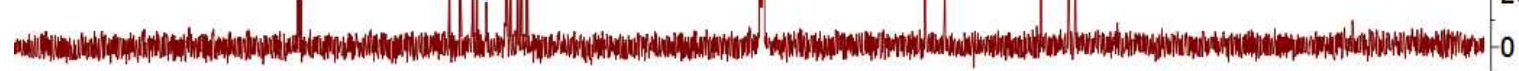

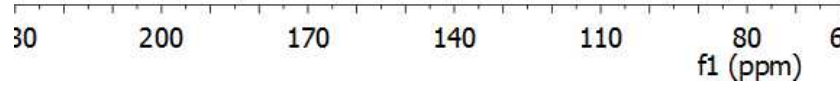



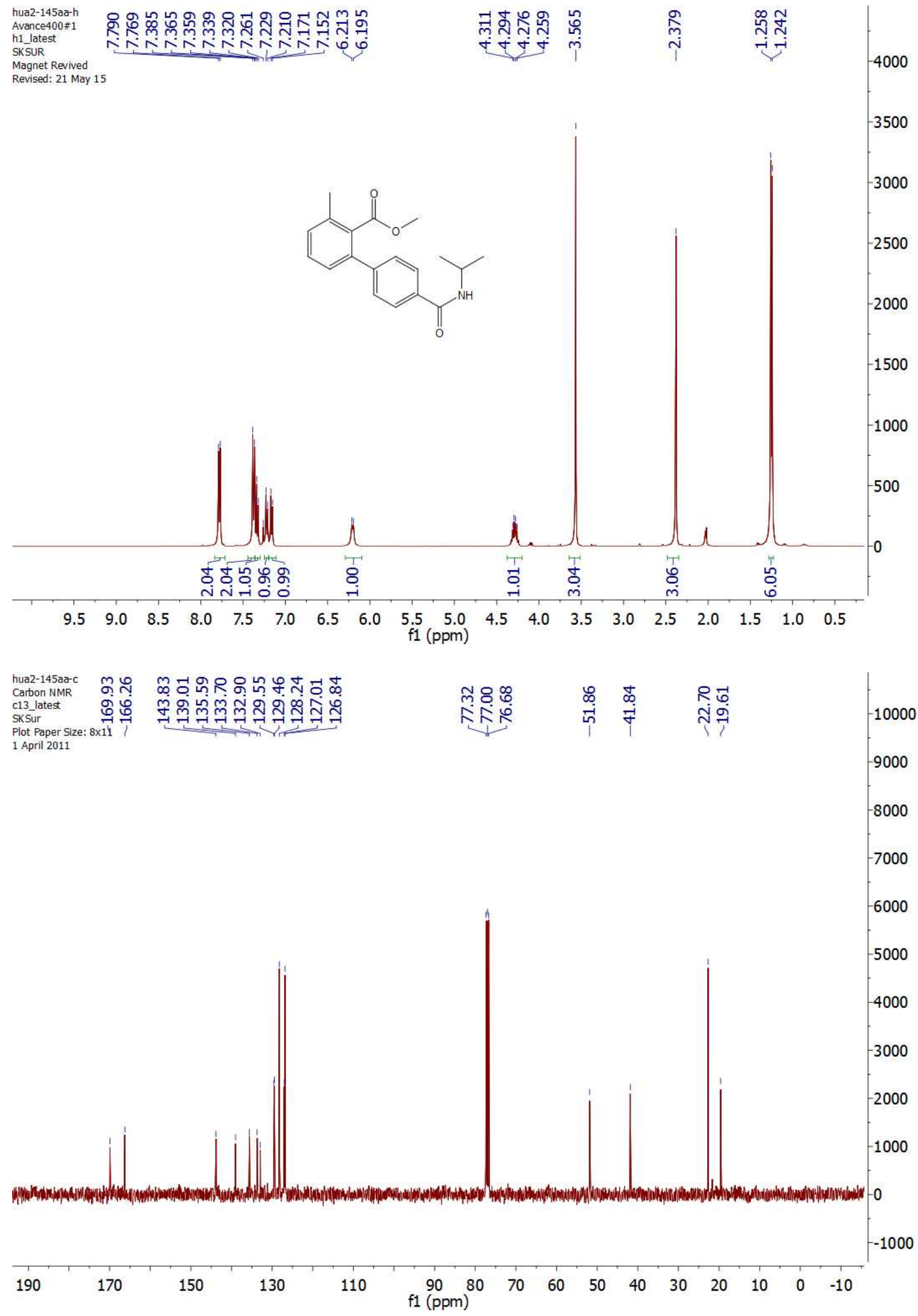

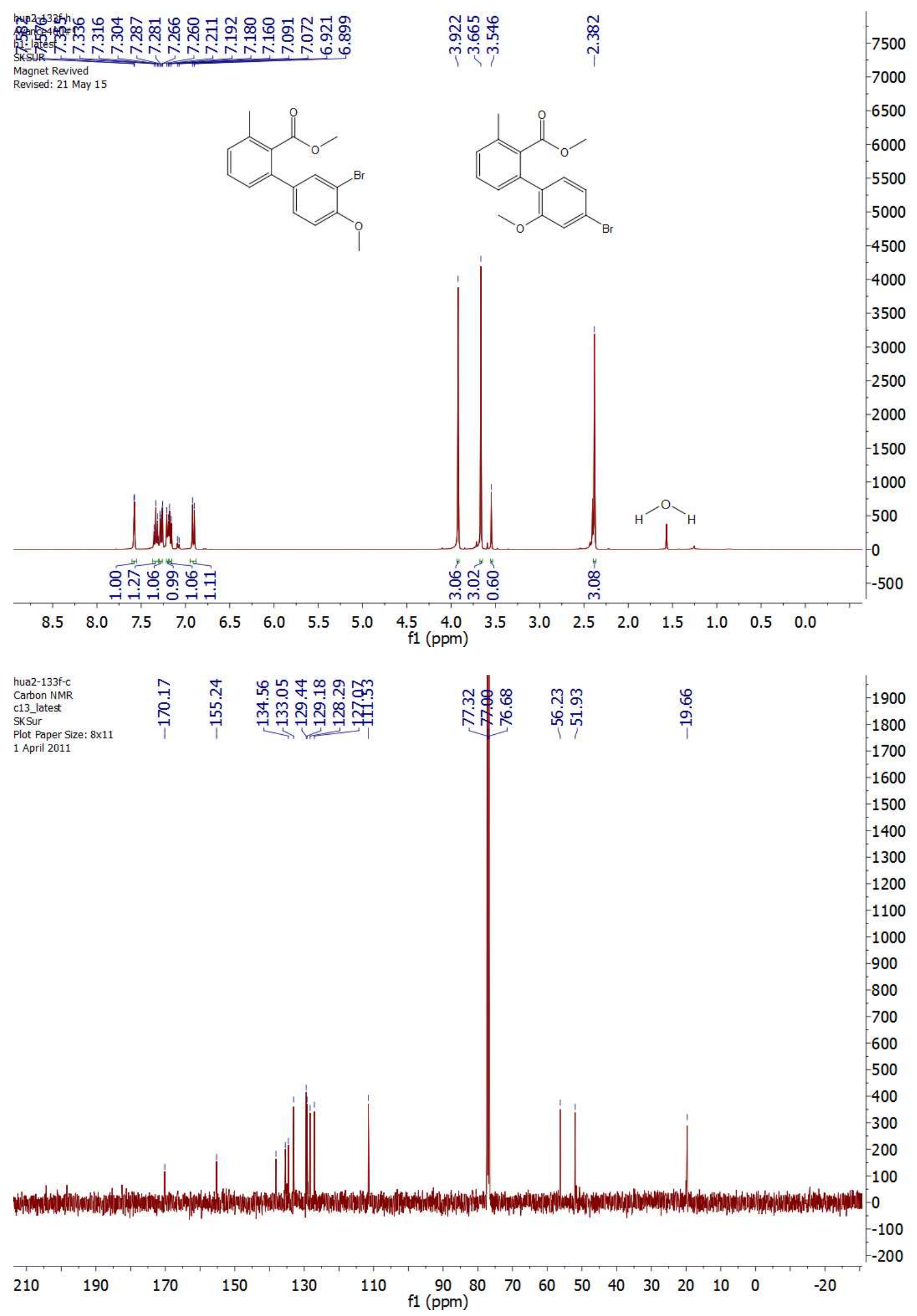

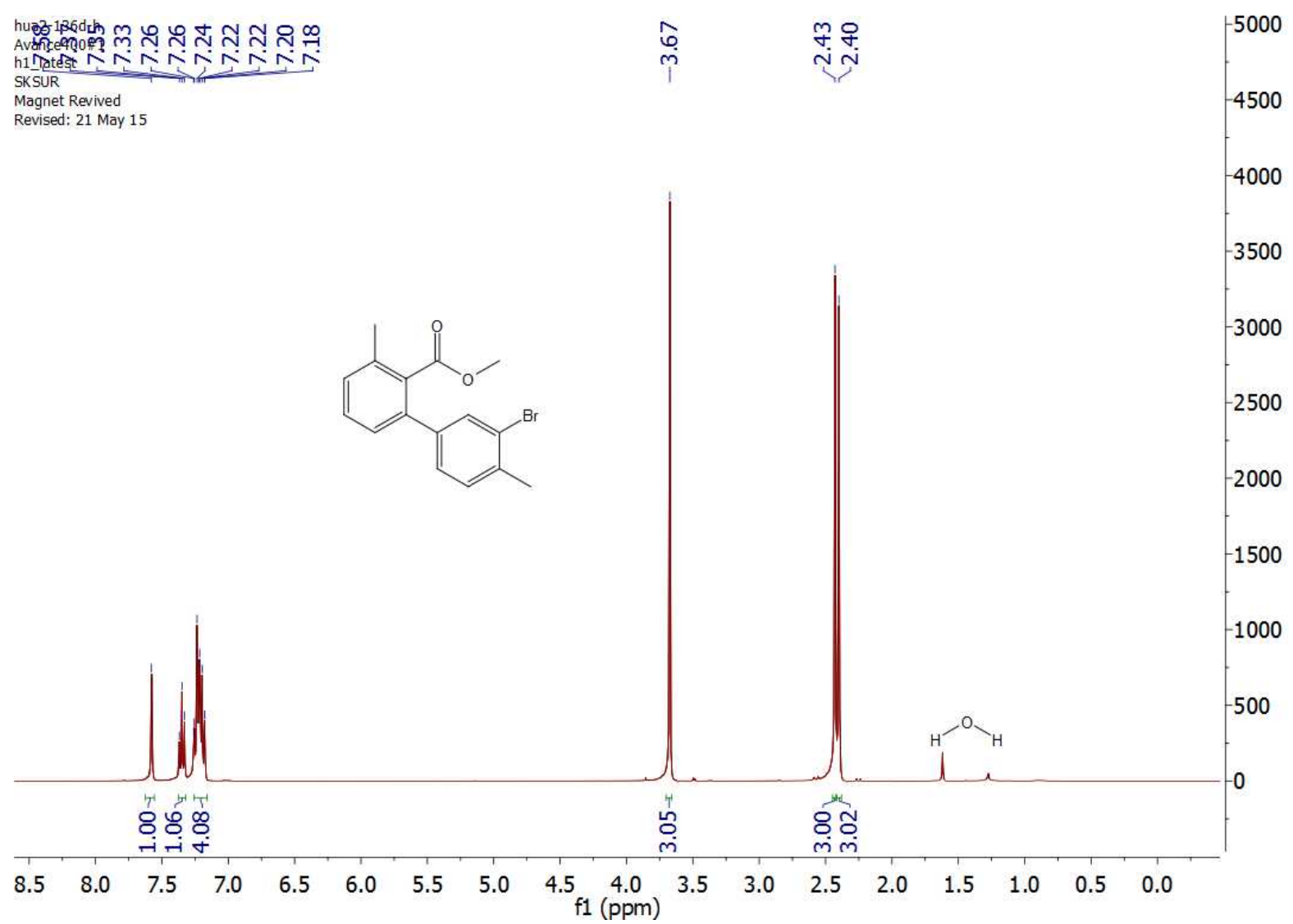

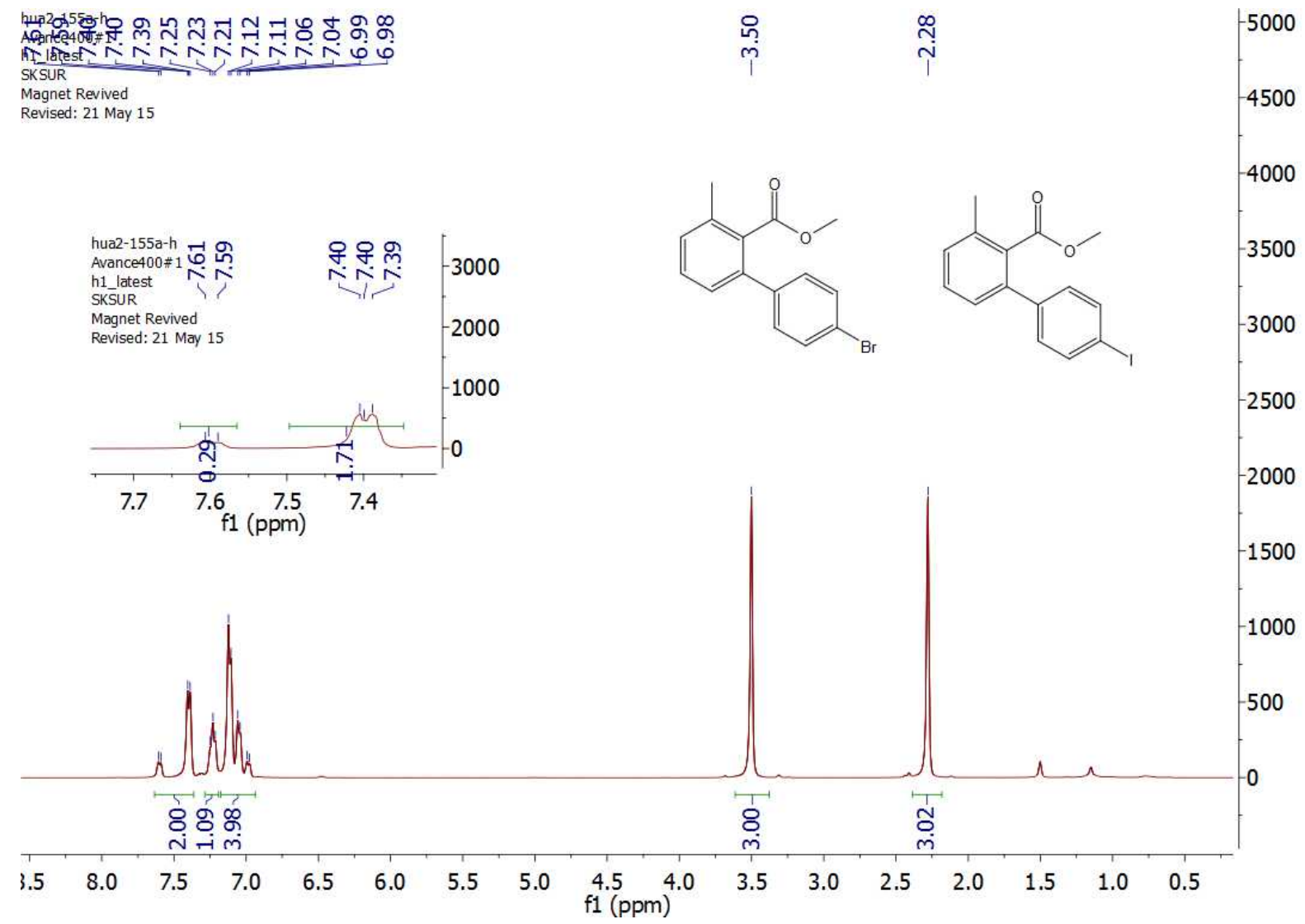

hua2-155a-c

Carbon NMR

C13_lates

Plot Paper Size: $8 \times 11$

1 April 2011

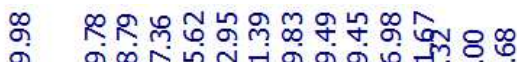

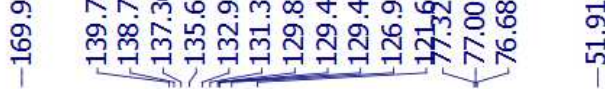

क़่

$-14000$

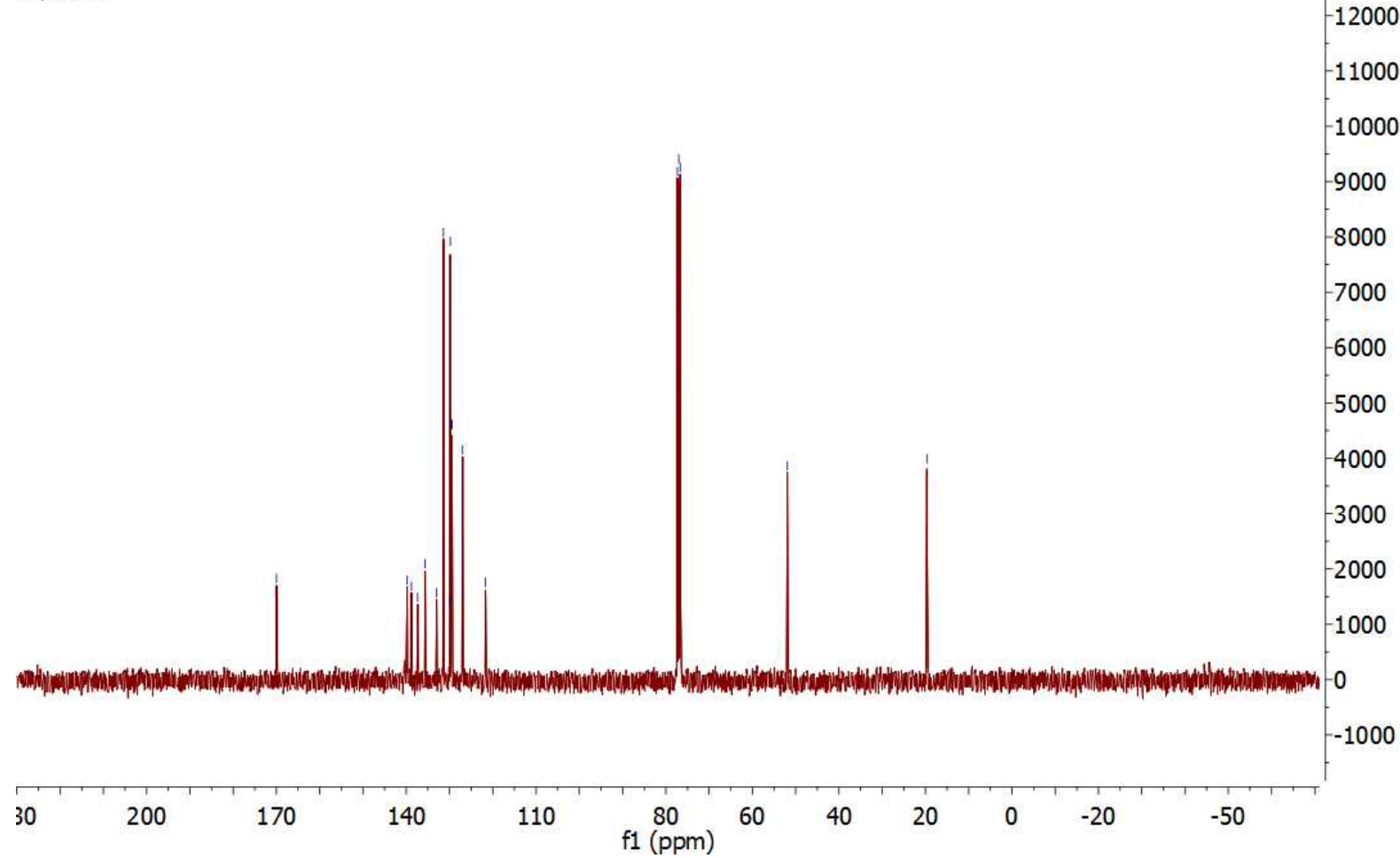


<smiles>Cc1cccc(-c2cccc(Br)c2)c1C(=O)O</smiles><smiles>Cc1cccc(-c2cccc(I)c2)c1C(=O)O</smiles>
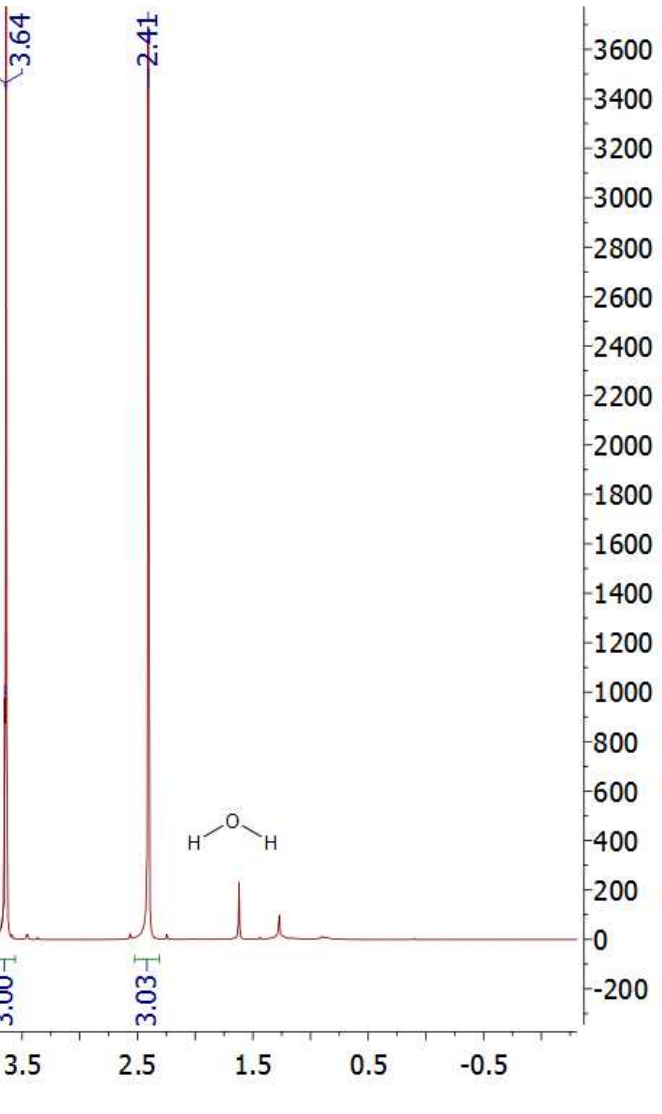

hua2-155b-c

Carbon NMR

C13_lates

Plot Paper Size: $8 \times 11$

1 April 2011

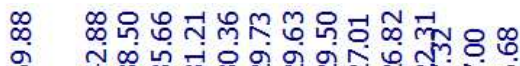

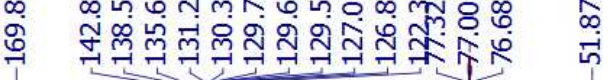

ڤิ)

$-8500$

$-8000$

$-7500$

$-7000$

$-6500$

$-6000$

$-5500$

5000

4500

4000

$-3500$

$-3000$

2500

2000

1500

1000

500

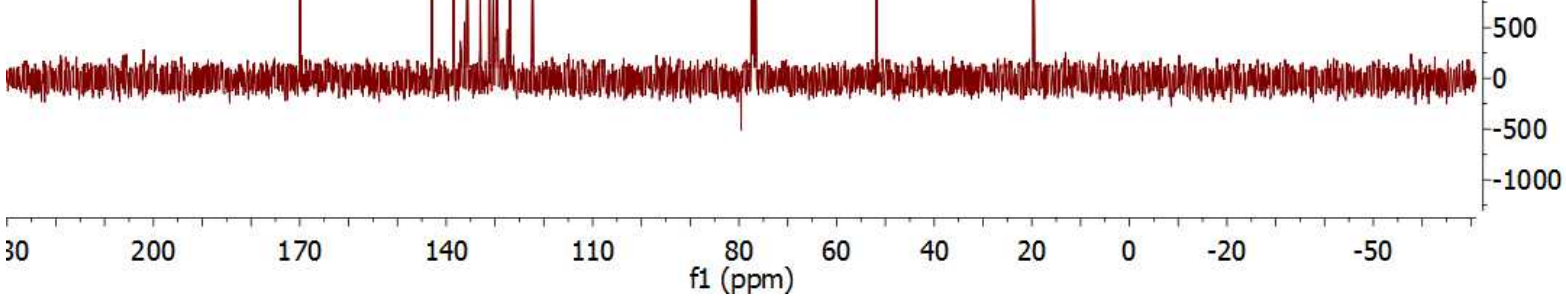




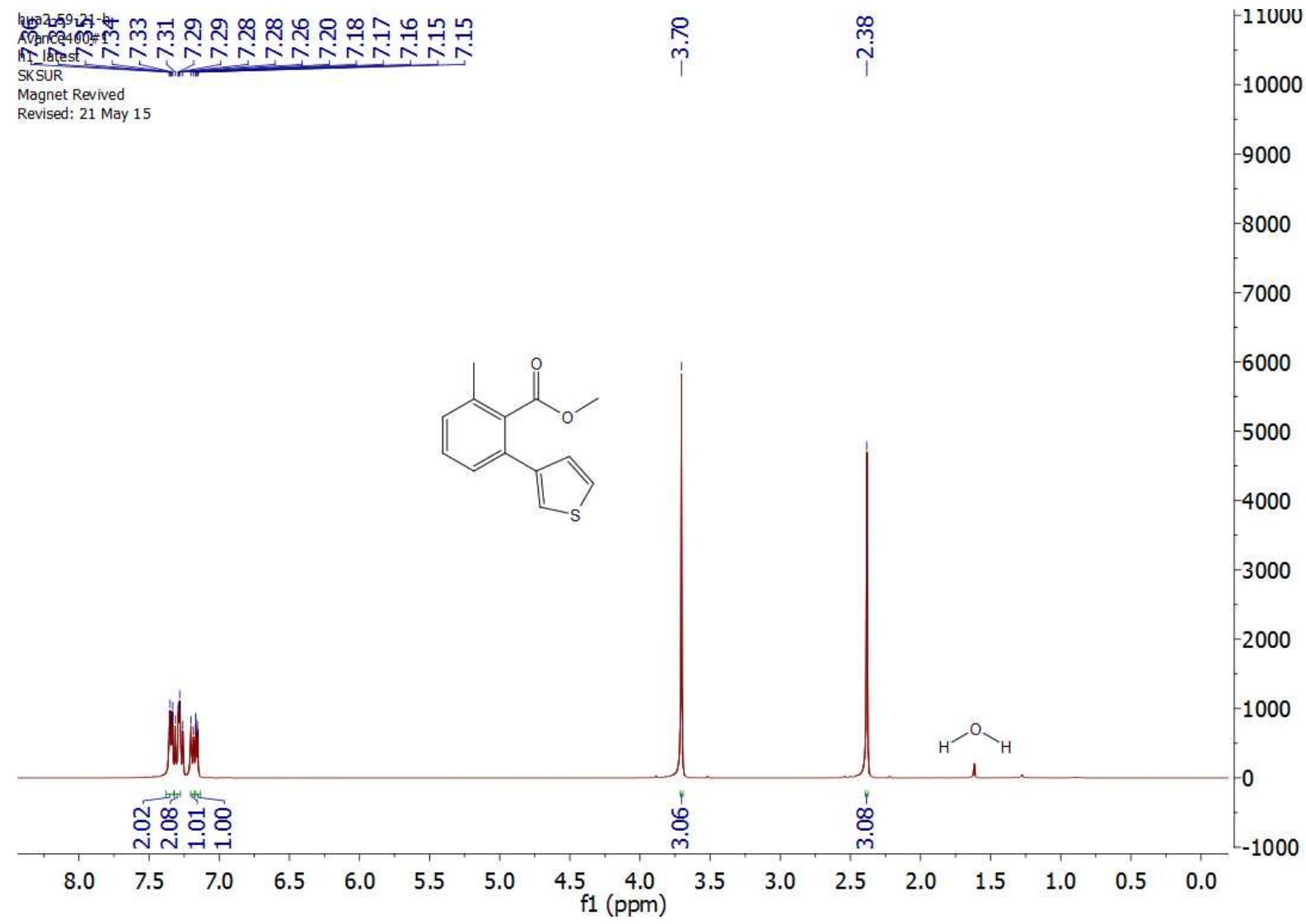

hua2-59-21-c Carbon NMR

C13_latest

Plot Paper Size: $8 \times 11$

1 April 2011

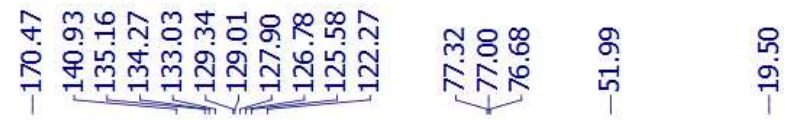

7000

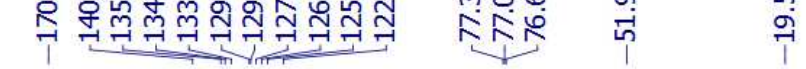

$-6500$

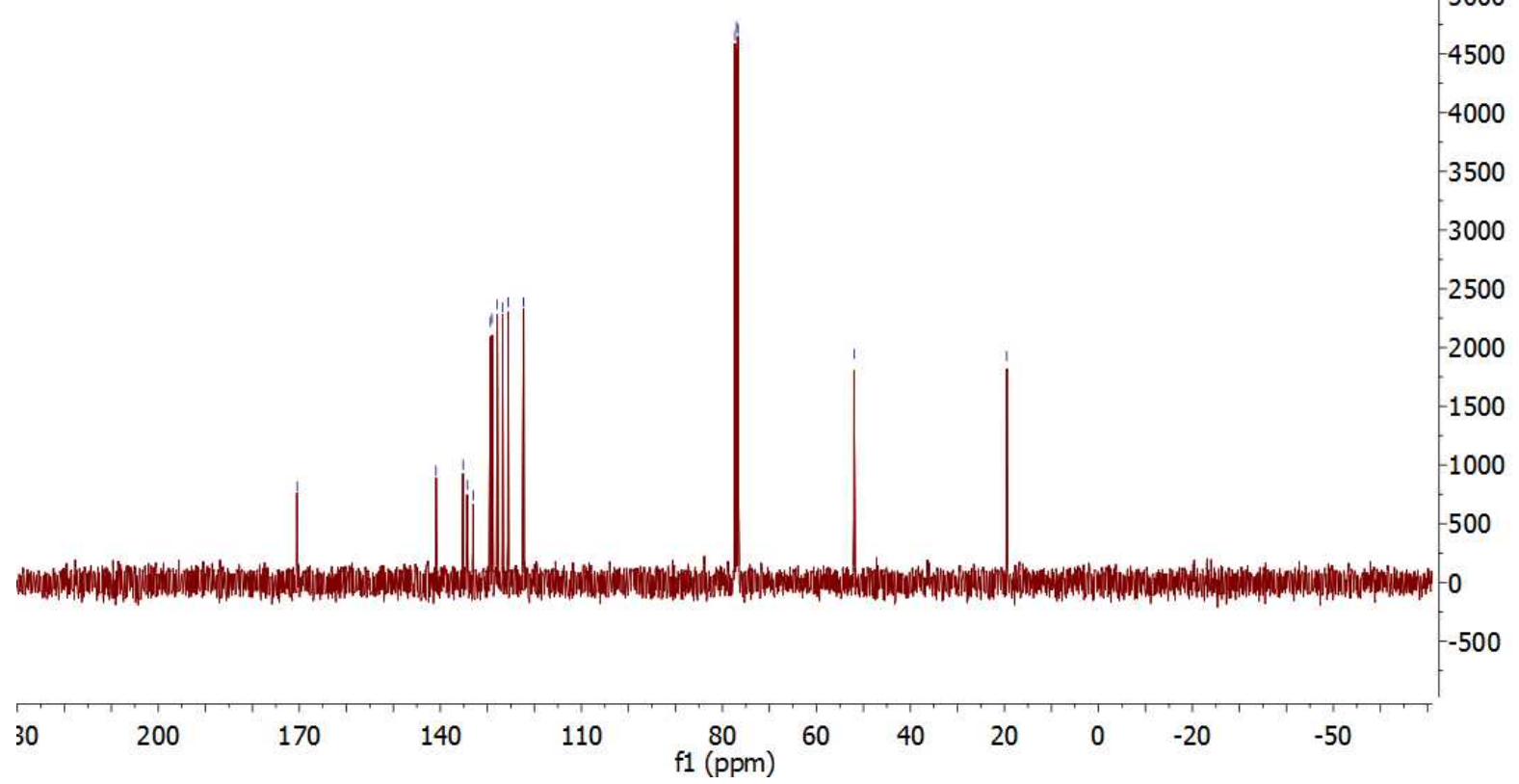




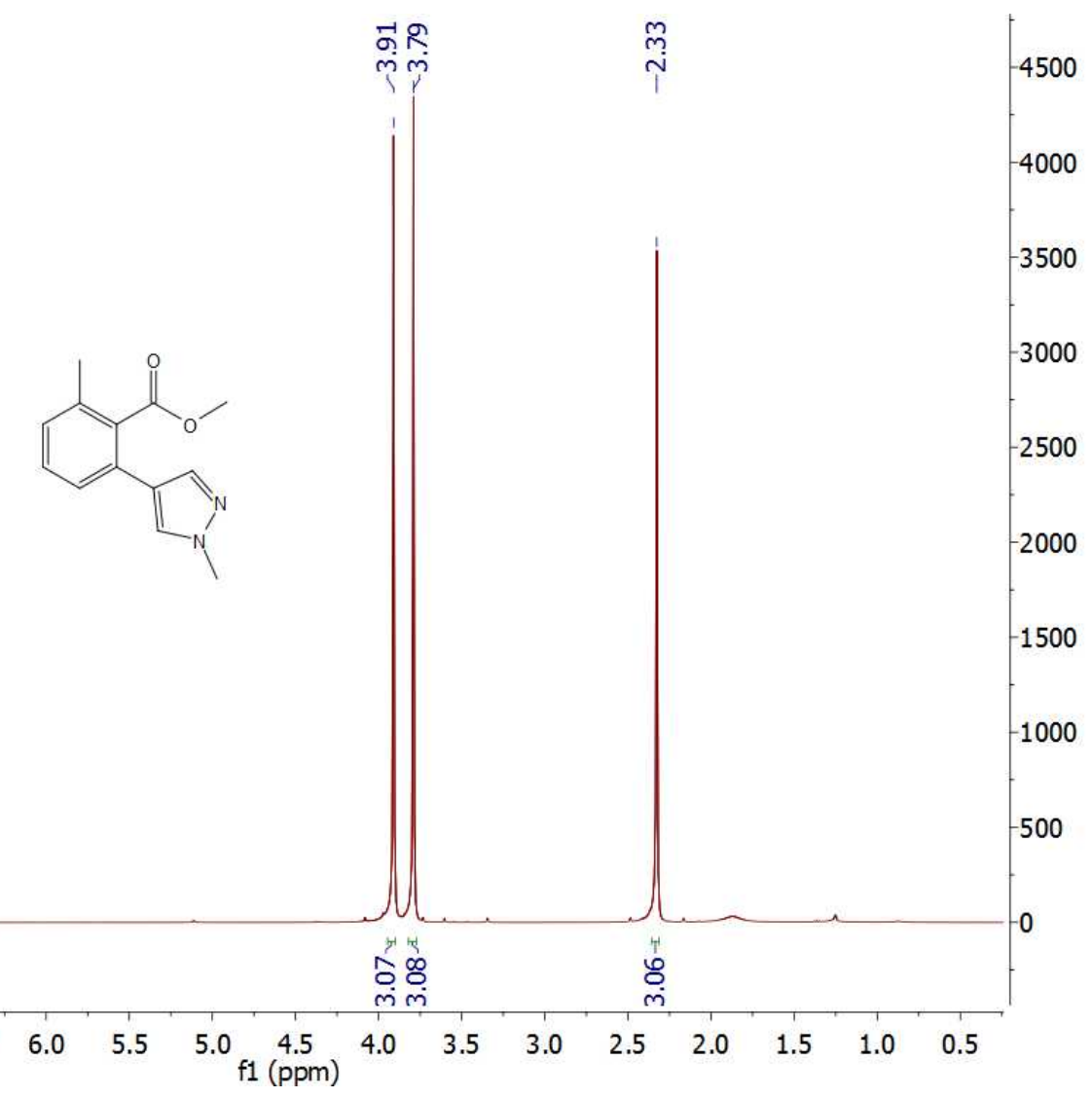

hua2-115a-c Carbon NMR

C13_latest

Plot Paper Size: $8 \times 11$

1 April 2011

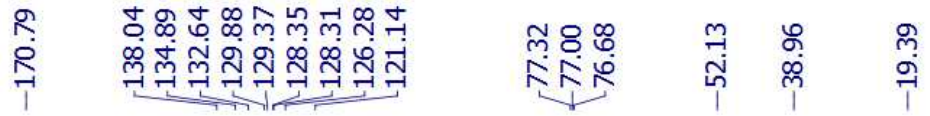

$-12000$

तन न्न

f1 (ppm)

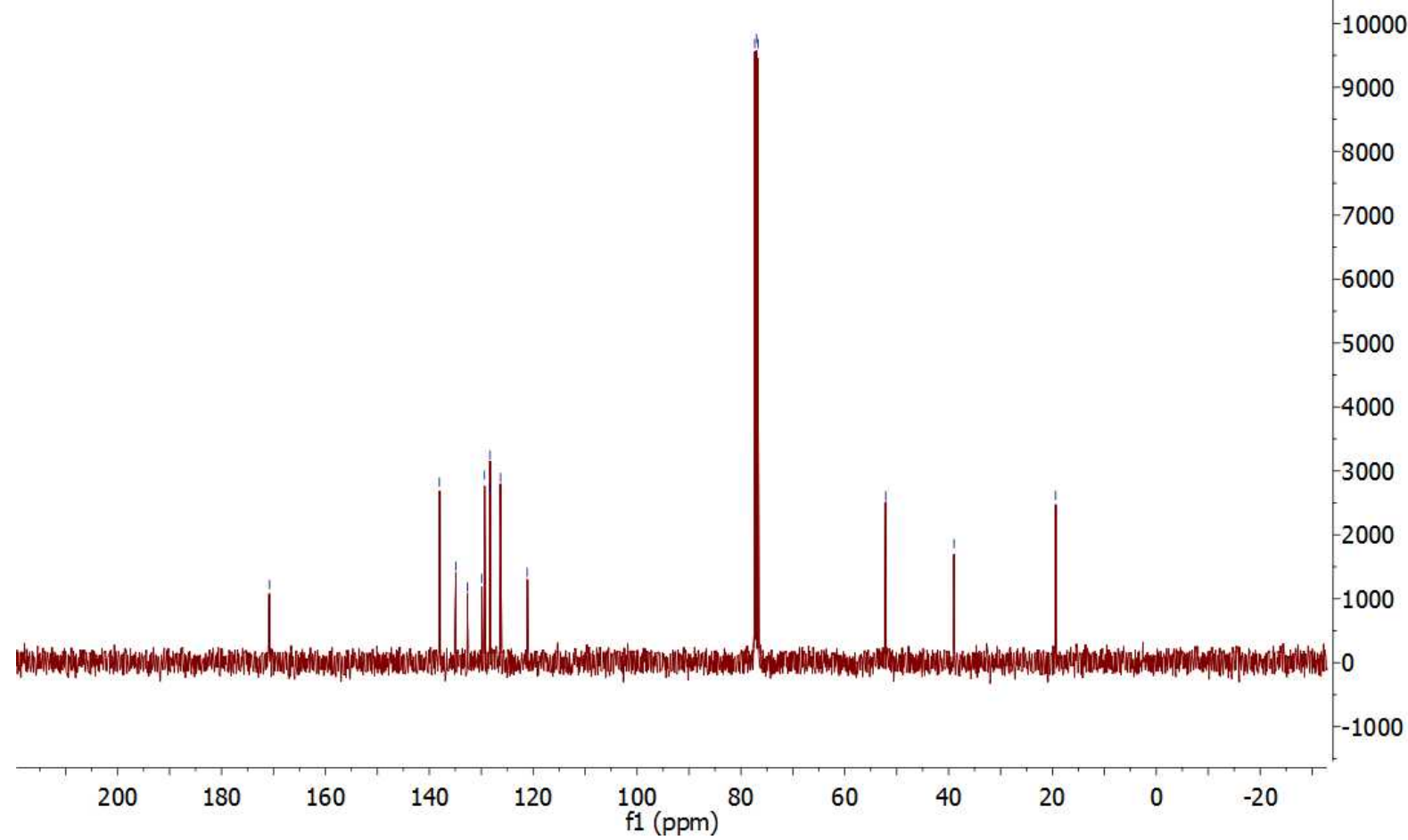


huaz154bu

hivatest N N N N N

Magnet Revived

Revised: 21 May 15

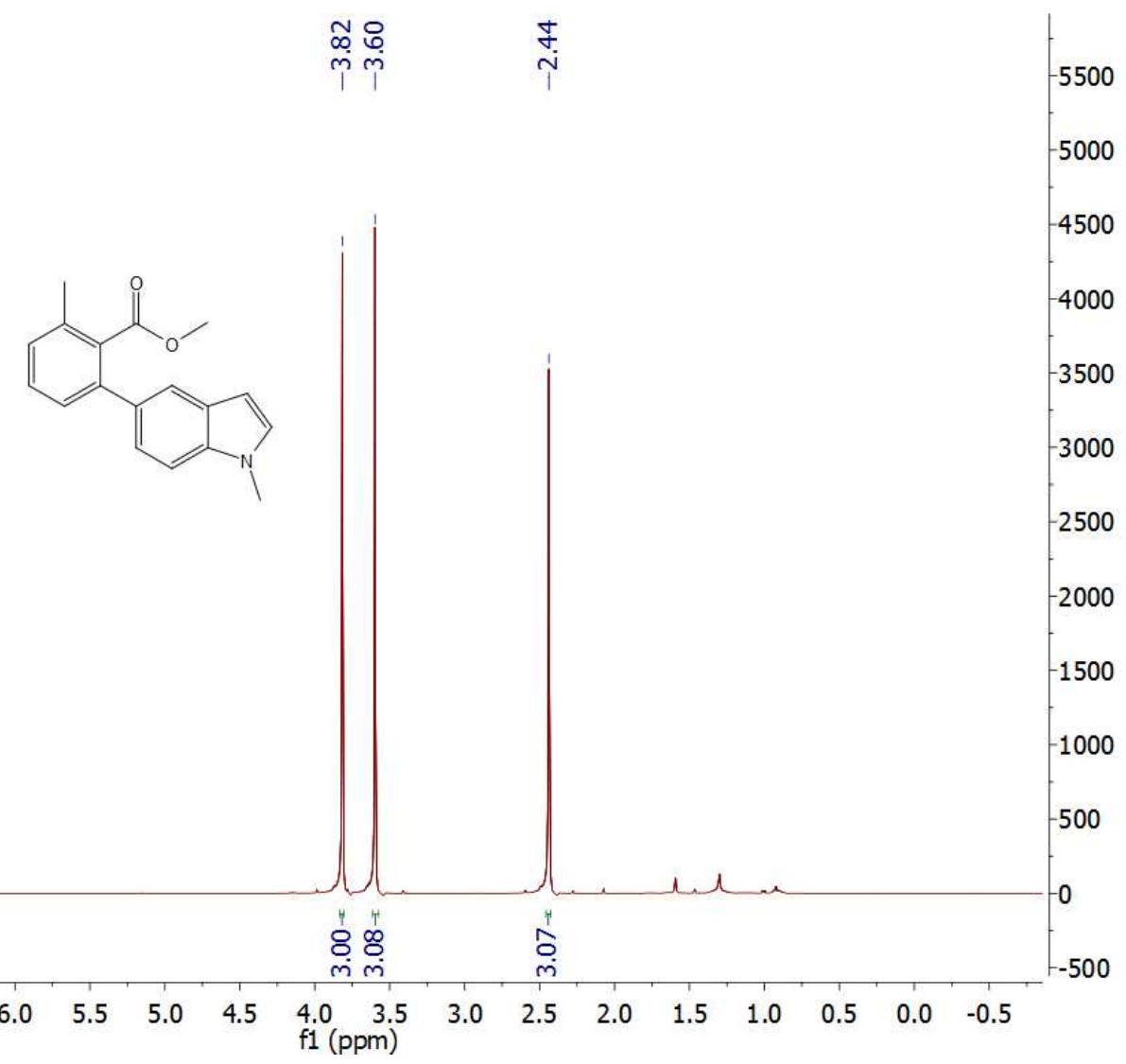

hya2-154b-c

C13 latest

ร न

SKSur

1 April 2011

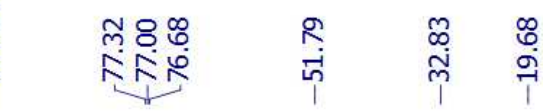

15000

14000

13000

12000

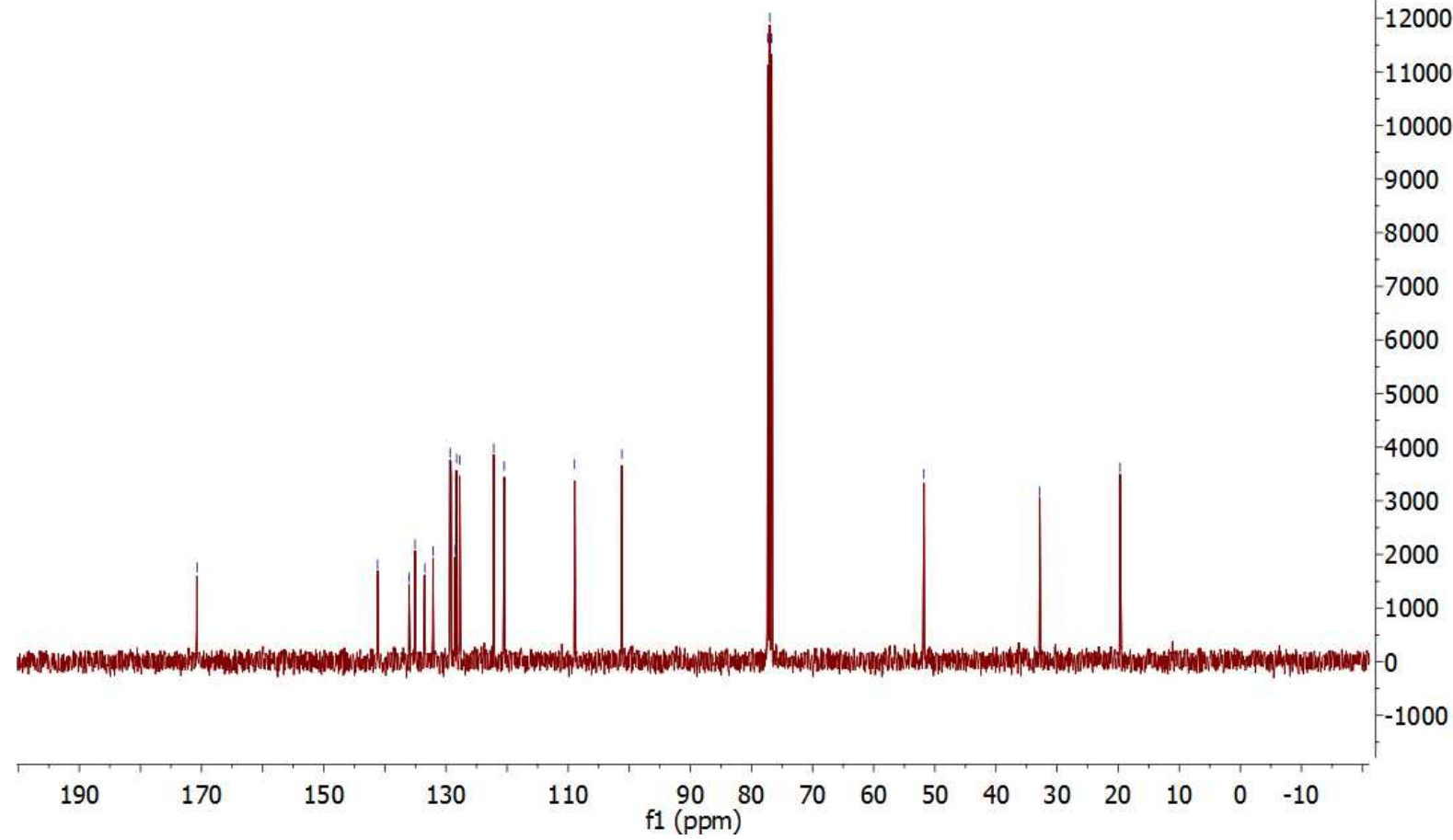


3v

hua2-1b-h

Avance $400 \# 1$

h1_latest
SKSUR

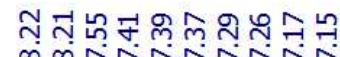

Magnet Revived

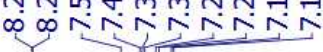

Revised: 21 May 15

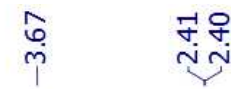

$-3400$

3200

3000

2800

2600

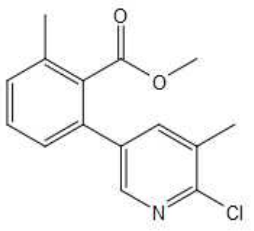

2400

2200

2000

1800

1600

1400

1200

1000

800

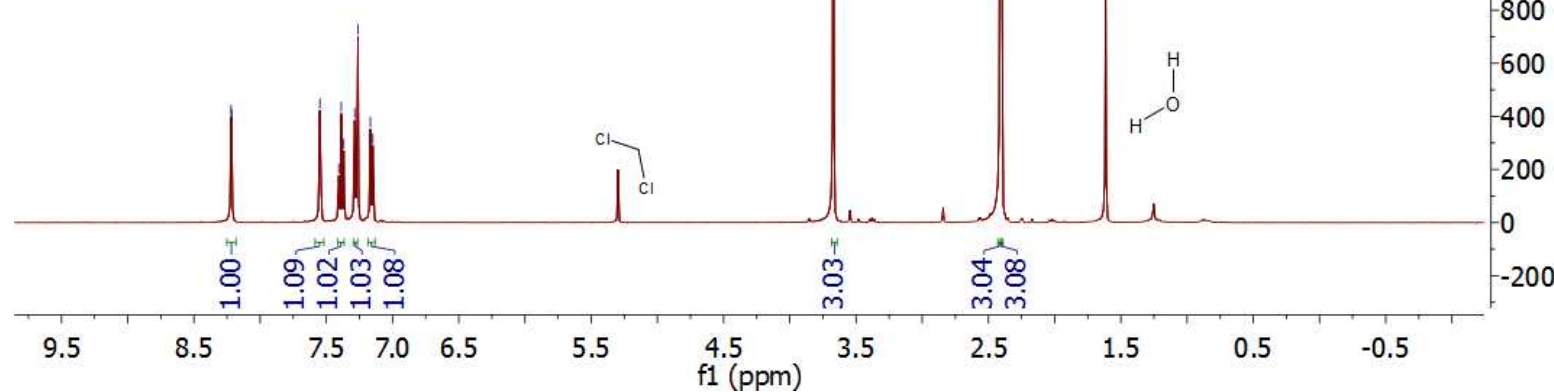

hua2-1b-c Carbon NMR C13_latest

Plot Paper Size: $8 \times 11$

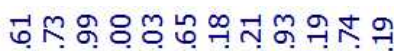

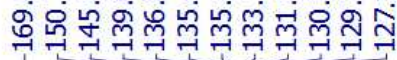

1 April 2011

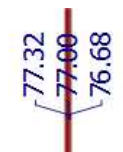

กิ่

ํํำ

$-19000$

$-18000$

$-17000$

$-16000$

$-15000$

$-14000$

$-13000$

$-12000$

$-11000$

$-10000$

9000

$-8000$

$-7000$

$-6000$

$-5000$

4000

3000

2000

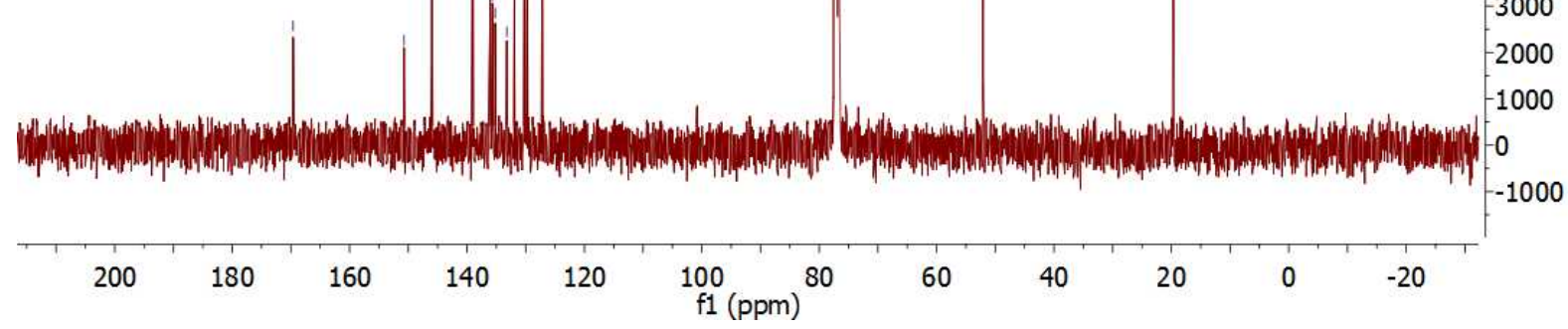


$3 w$

hua2-138-h

Avance $400 \# 1$
h1_latest

SKSUR

Magnet Revived

mon

i

Revised: 21 May 15

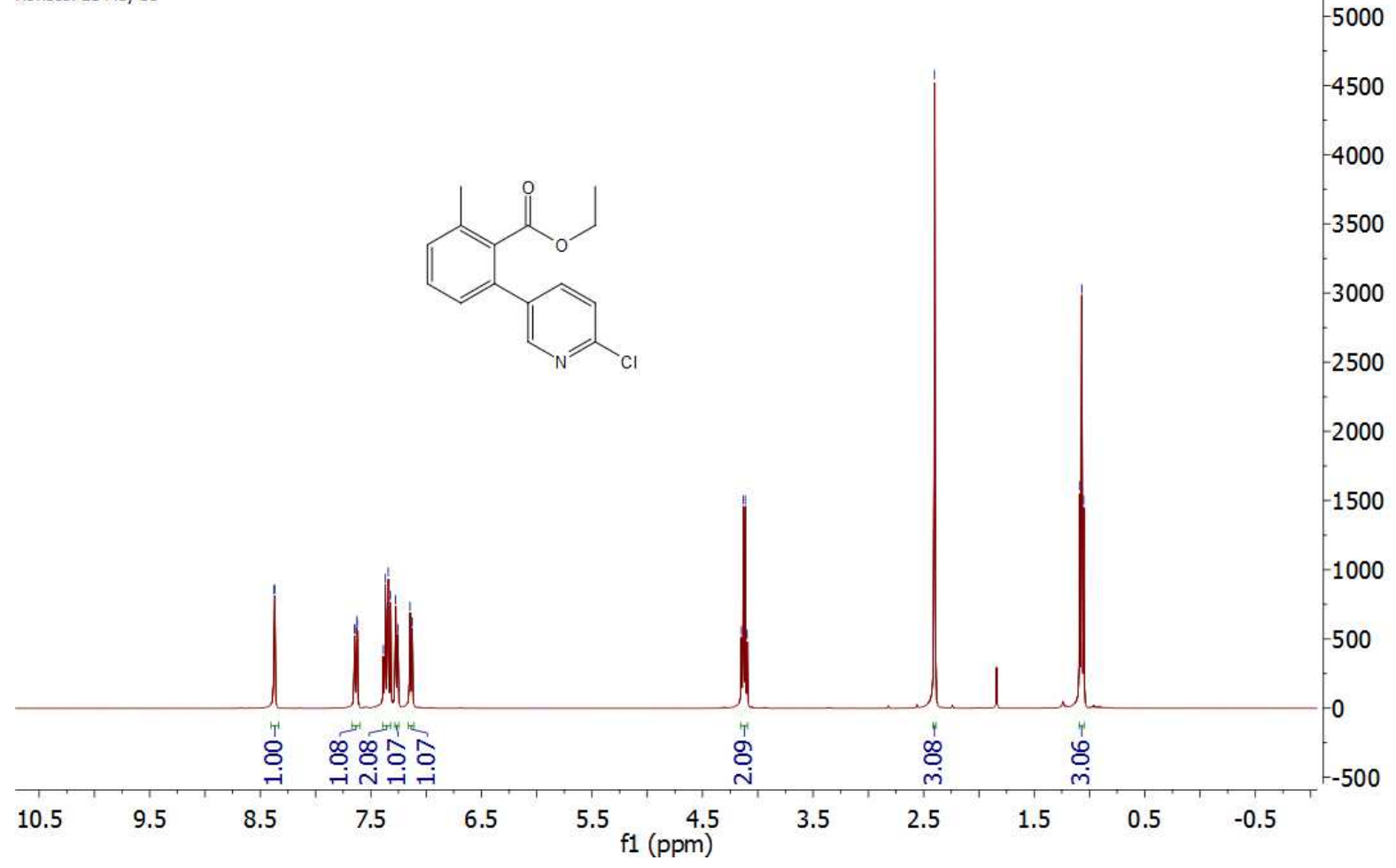

hua2-138-c

Carbon NMR

C13_latest

SKSur
Plot Paper Size: $8 \times 11$

1 April 2011

ஸ

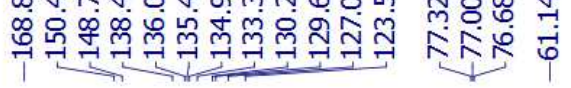

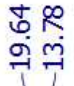

$5 / 000$

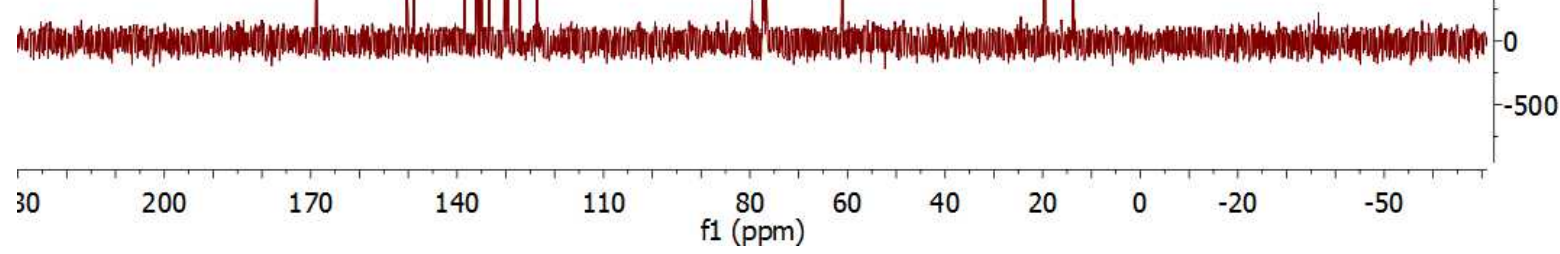




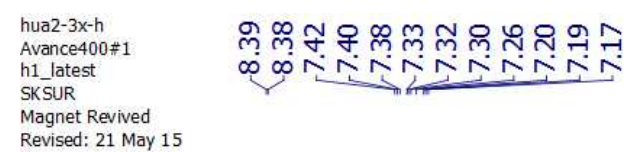

$3 \mathbf{x}$
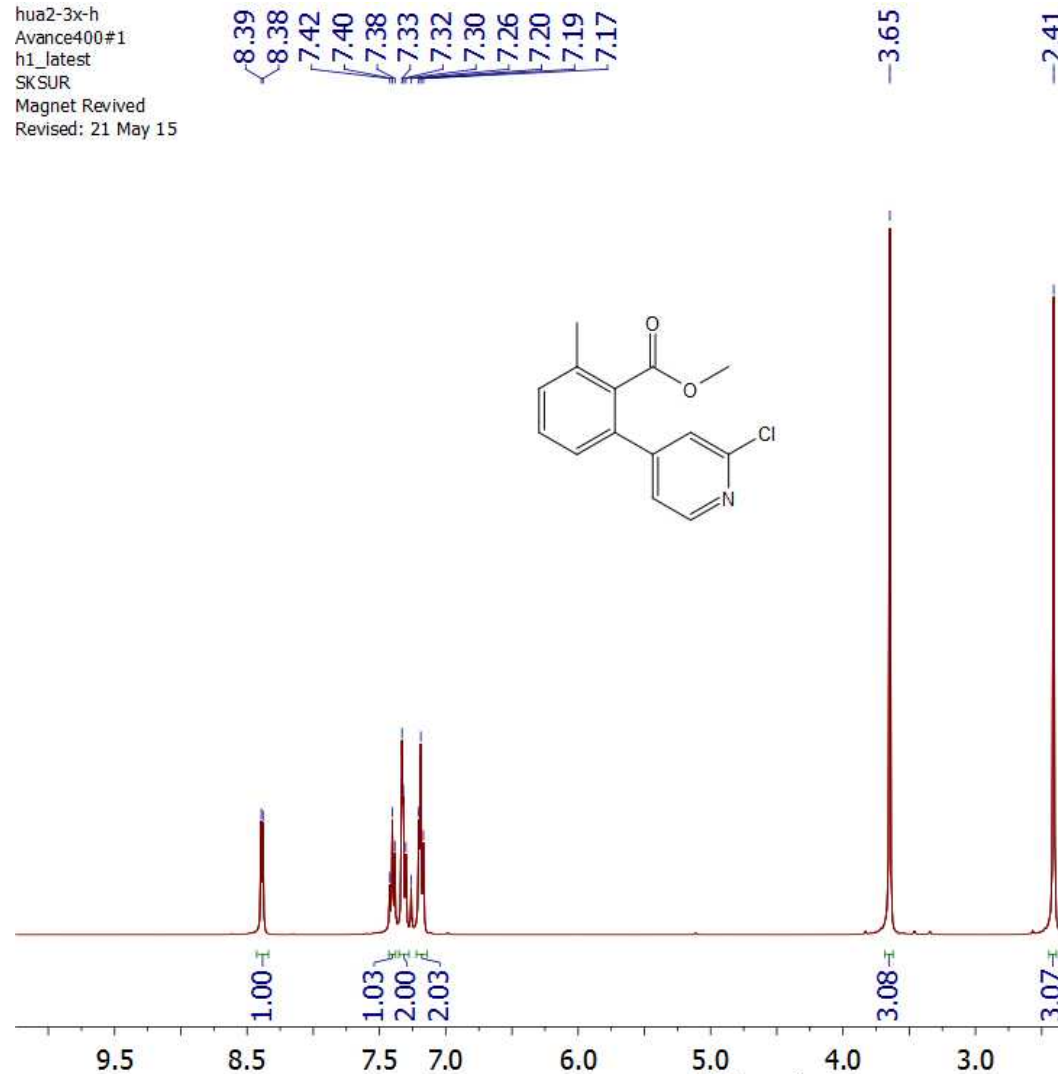

hua2-160c-c

Carbon NMR

SKSur

Plot Paper Size: $8 \times 11$

1 April 2011

8.5

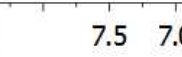

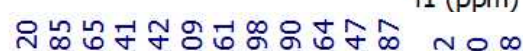

舟

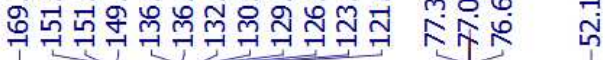

$\underset{\substack{1 \\ 0}}{\substack{5 \\ 0}}$

650

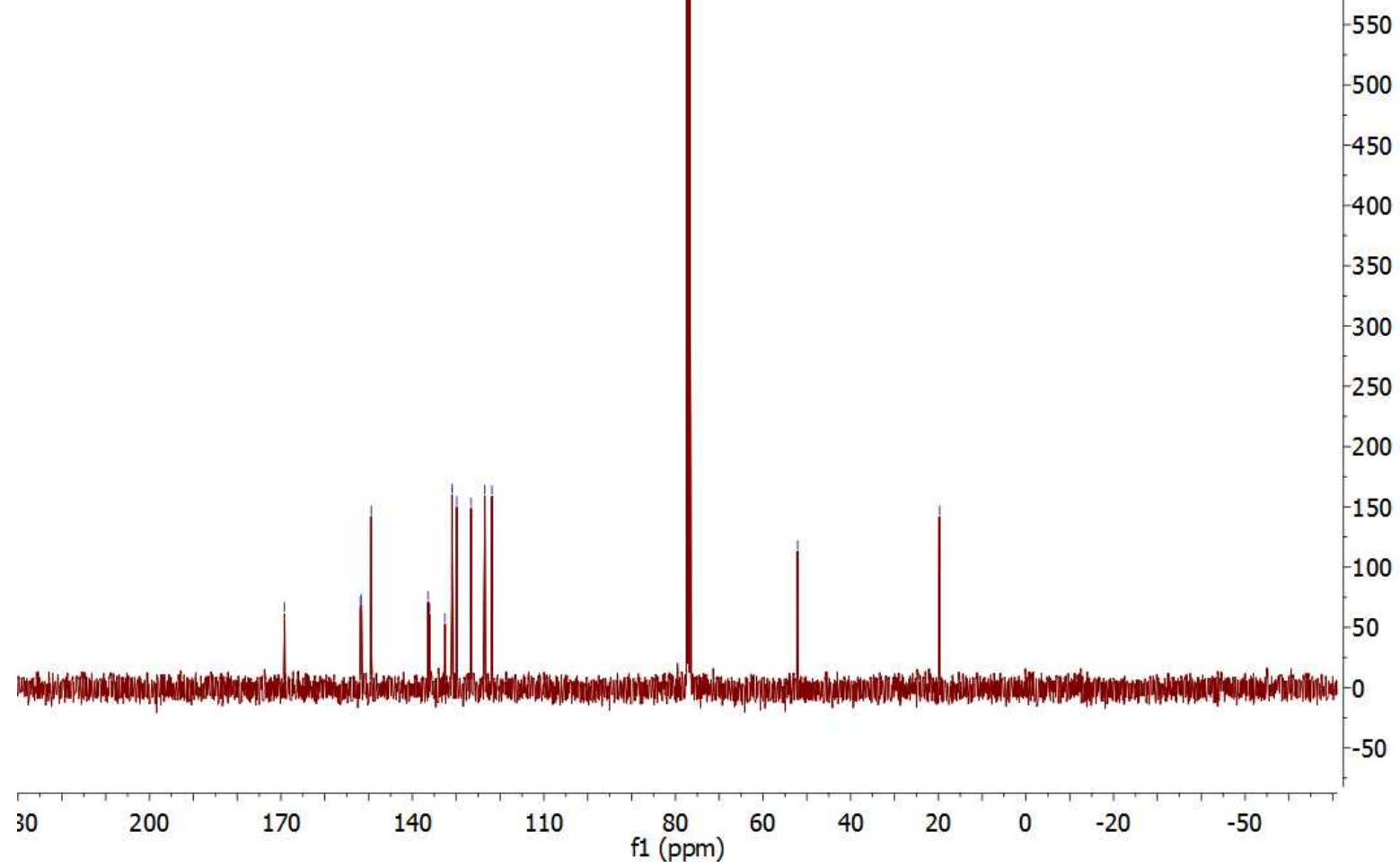


$3 y$

hua2-160a-h

Avance400\#

SKSUR

Magnet Revived

Revised: 21 May 15

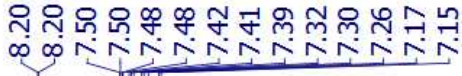

:̊ํํ

î

\begin{tabular}{ll|l} 
운 & -5000
\end{tabular}<smiles>COC(=O)c1c(C)cccc1-c1cnc(Cl)c(F)c1</smiles>

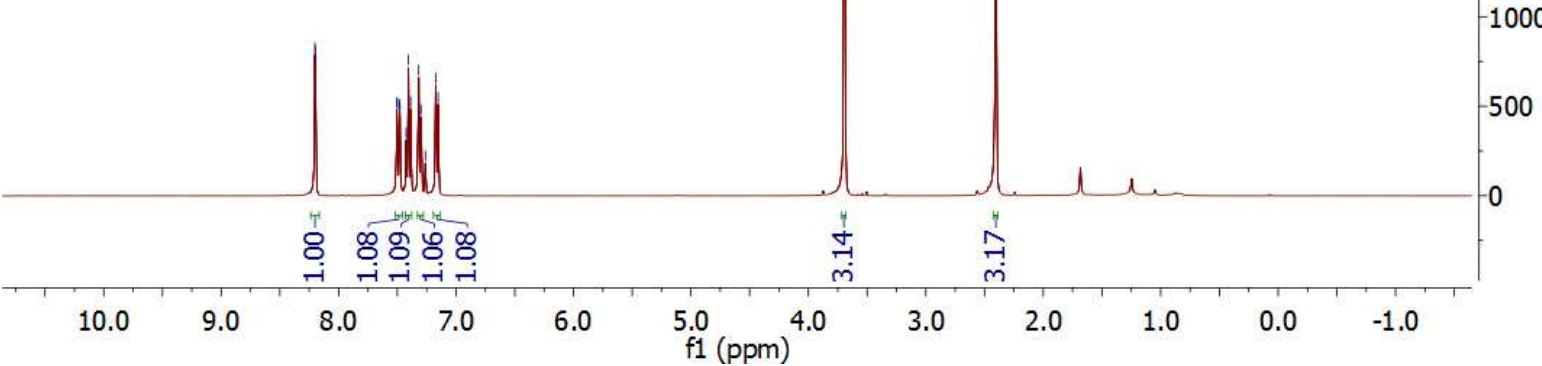

hua2-160a-c

Carbon NMR

C13_latest

Plot Paper Size: 8x11

1 April 2011

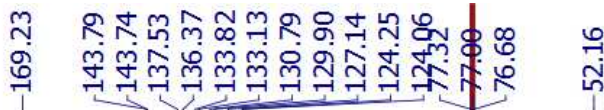

กับ

$\stackrel{2}{\stackrel{2}{7}}$

osue

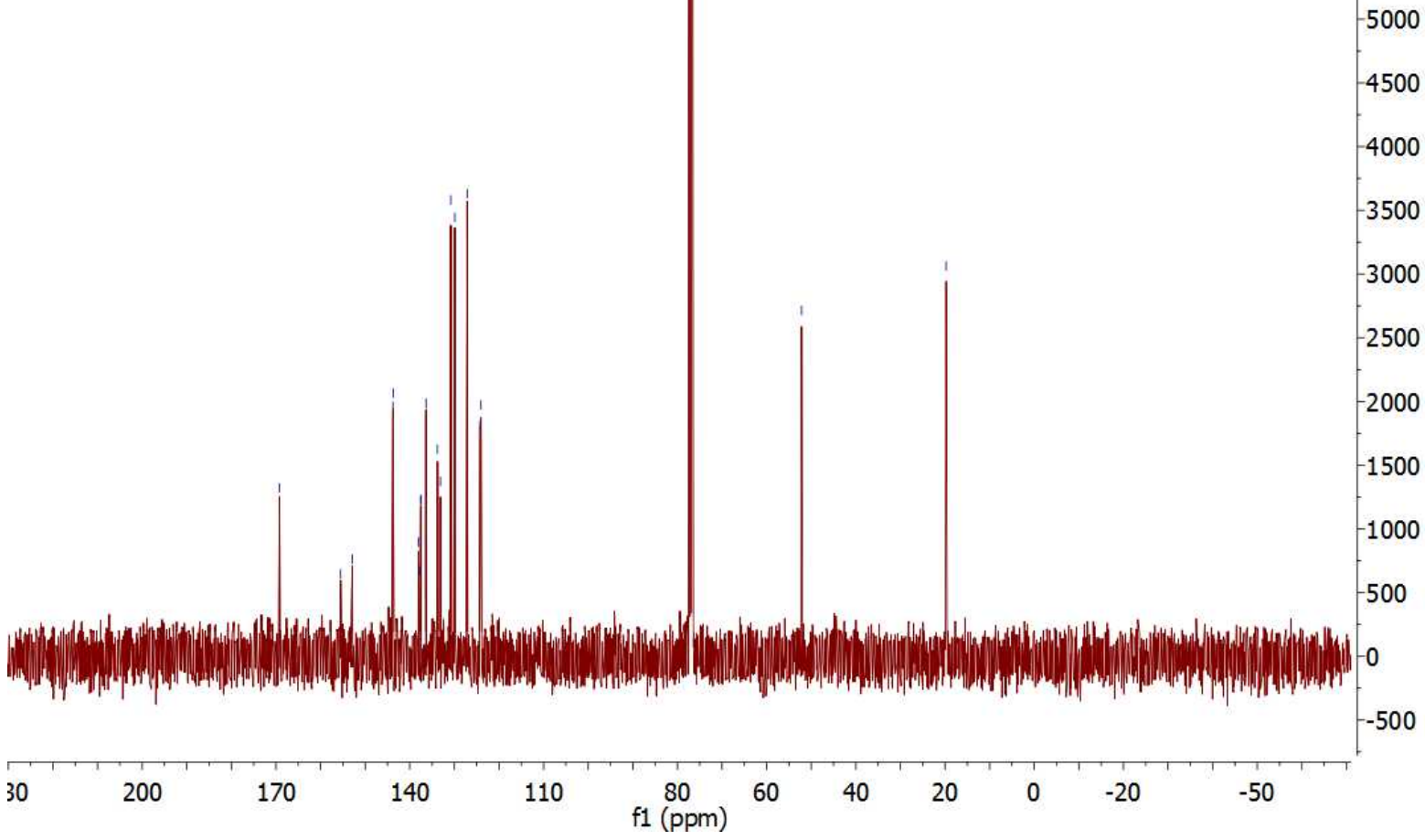




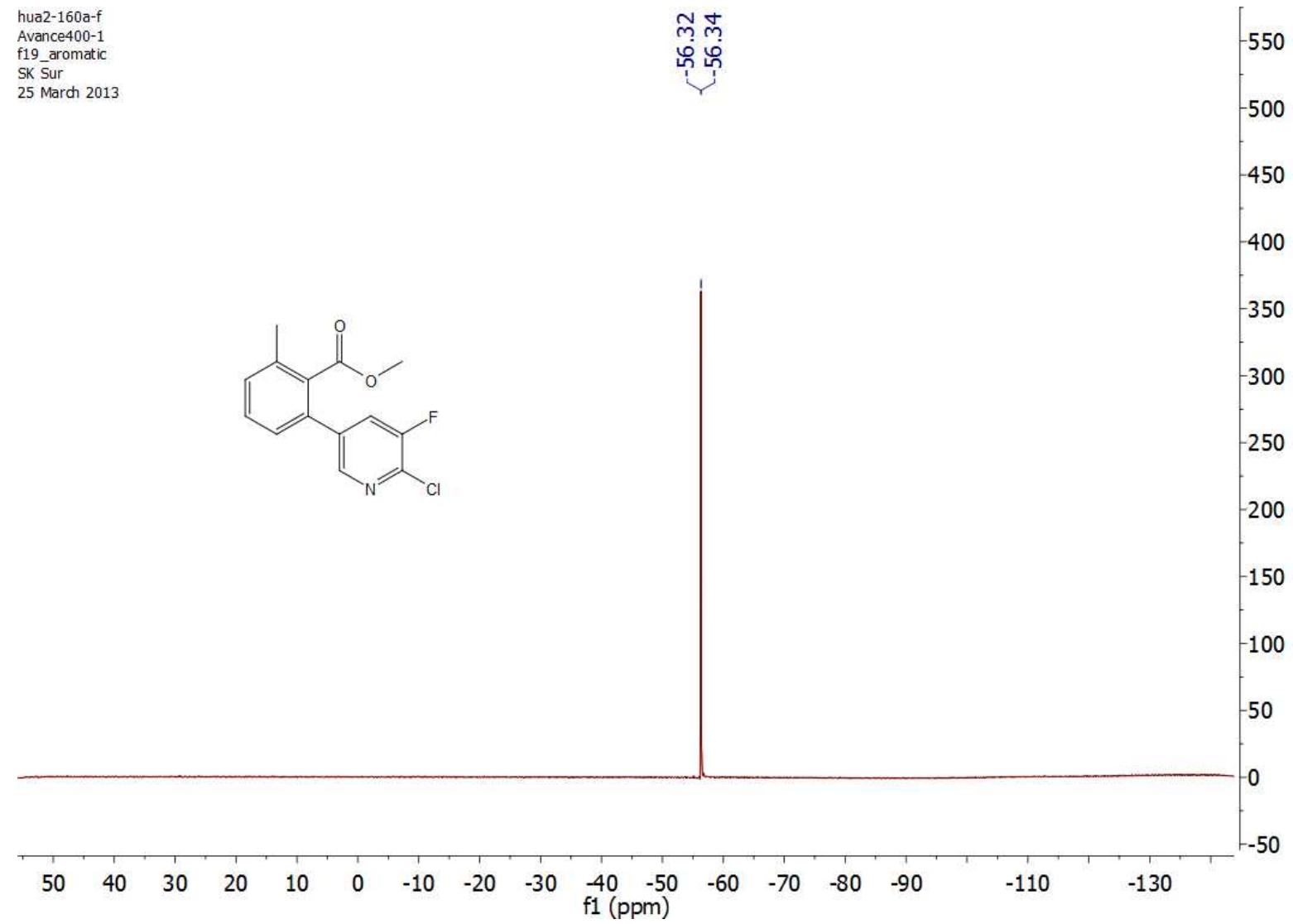


3z

hua2-160d-h
Avance400\#1
h1_latest
SKSUR
Magnet Revived

Revised: 21 May 15

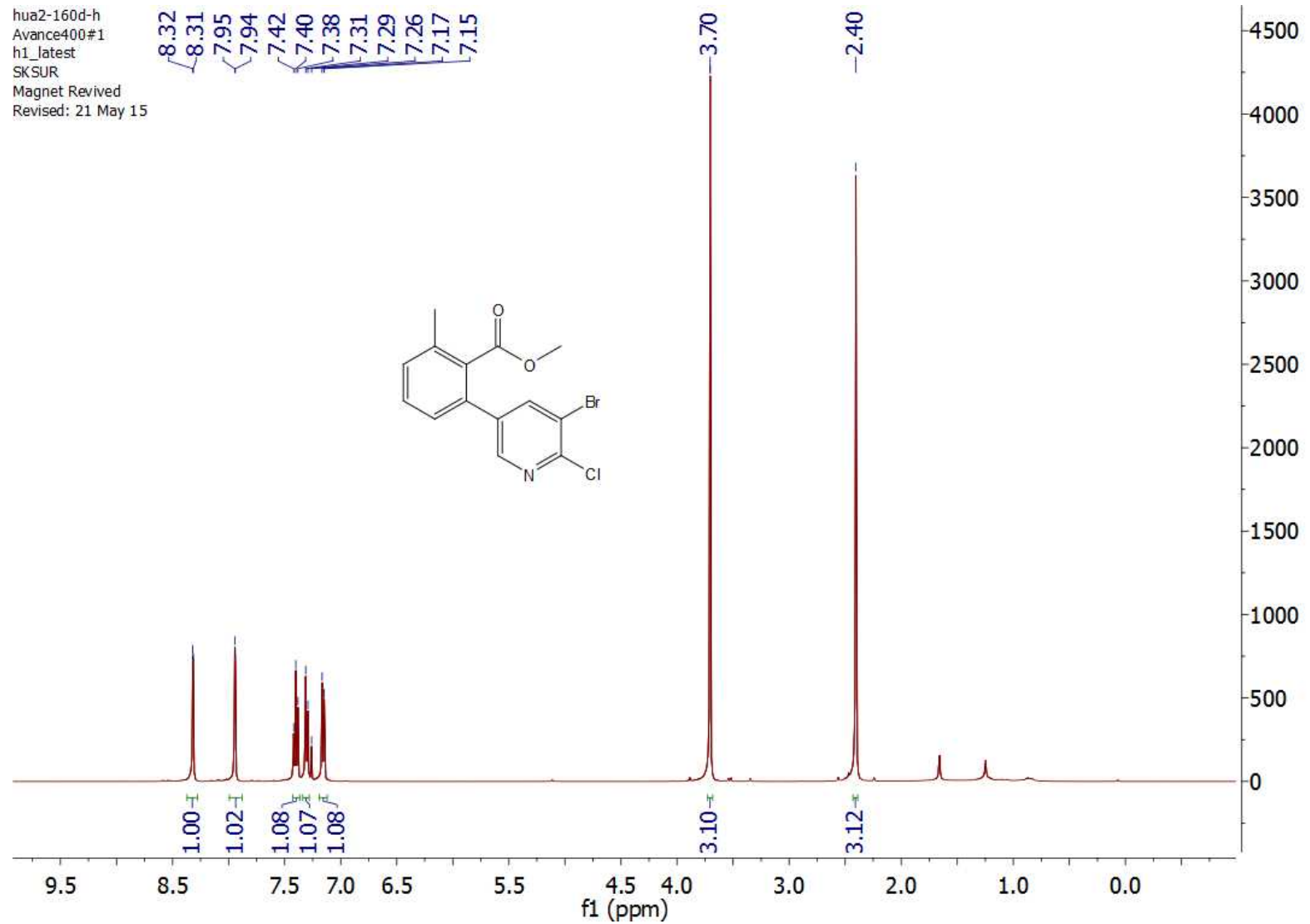

hua2-160d-c त)

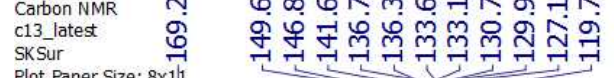

1 April 2011

1 (ppm)

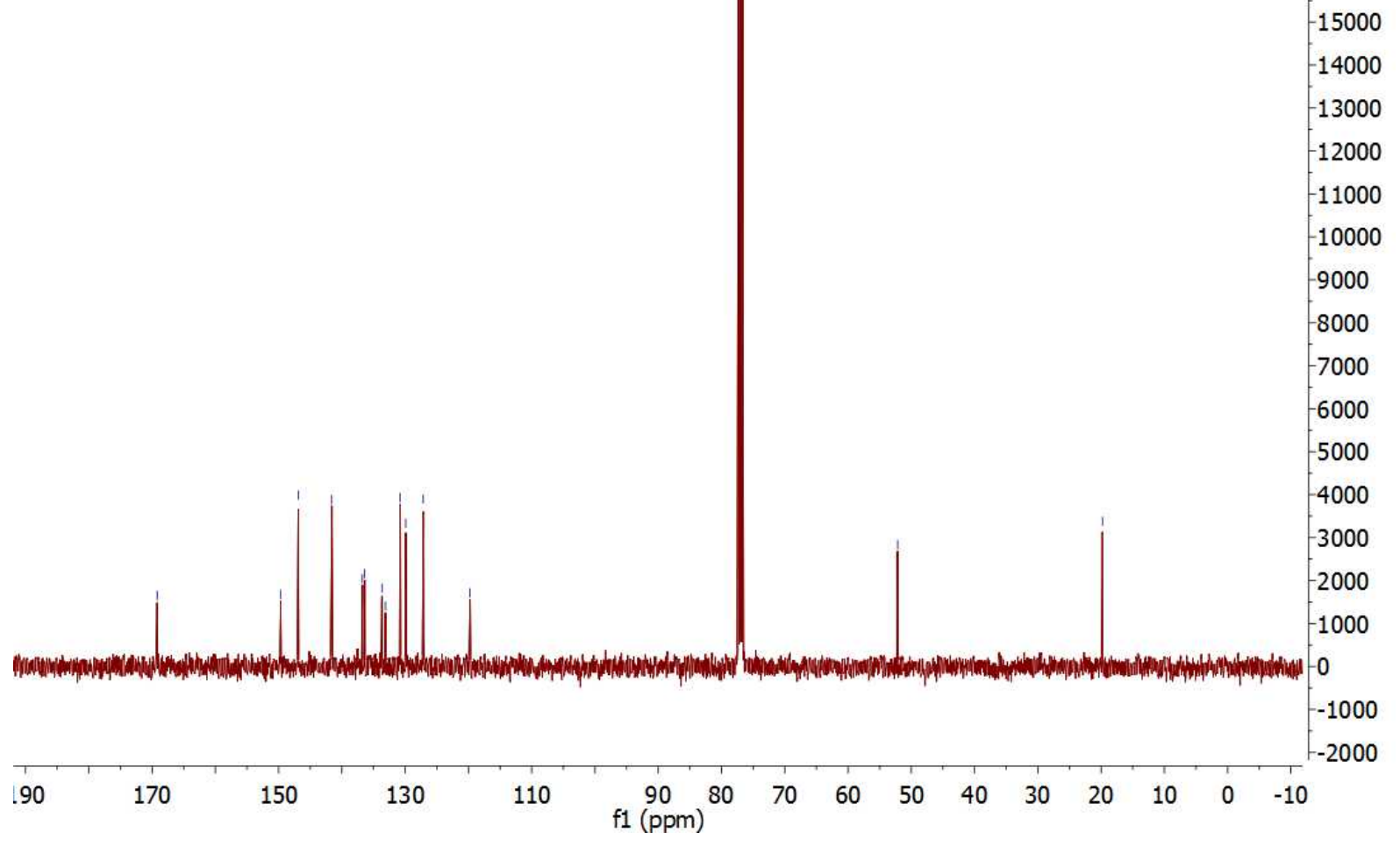


$4 b a$

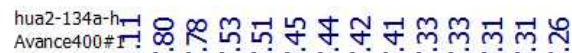

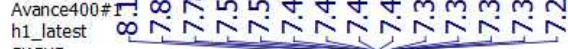

SKSUR

Magnet Revived

Revised: 21 May 15

$\stackrel{\infty}{\infty}$

$-8500$

$-8000$

7500

7000

(1)

$-6500$

6000

$-5500$

$-5000$

4500

4000

$-3500$

3000

2500

2000

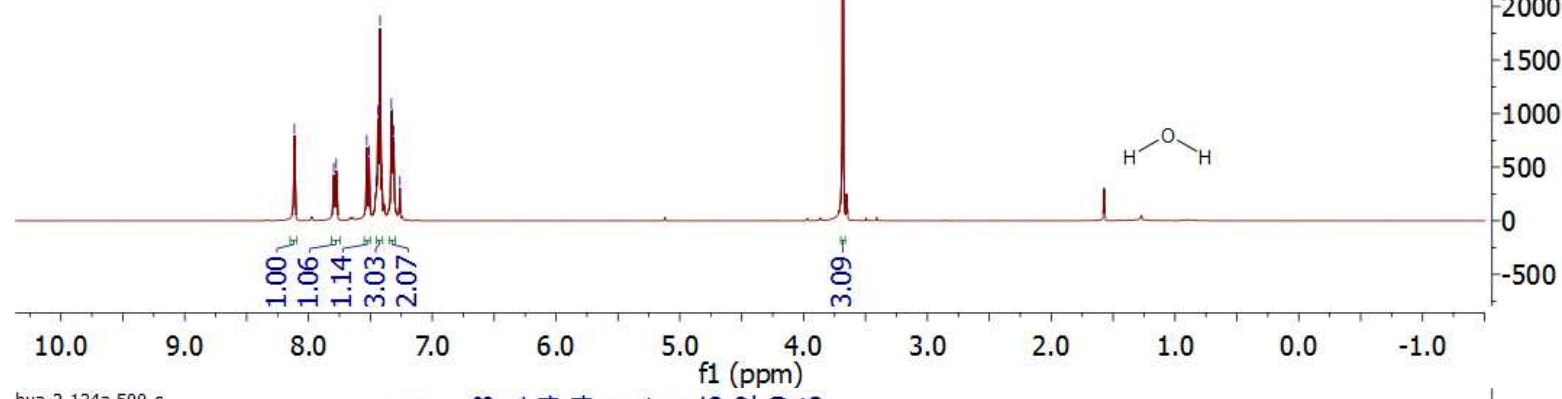

hua-2-134a-500-c

Avance 500
Carbon NMR: c13_latest

Sur/ 27 april 16

CD d3, $125.78 \mathrm{MHz}$, 25deg

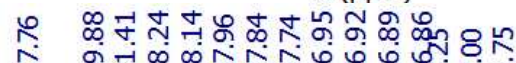

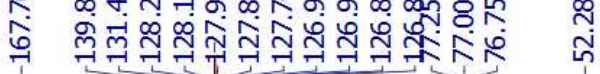
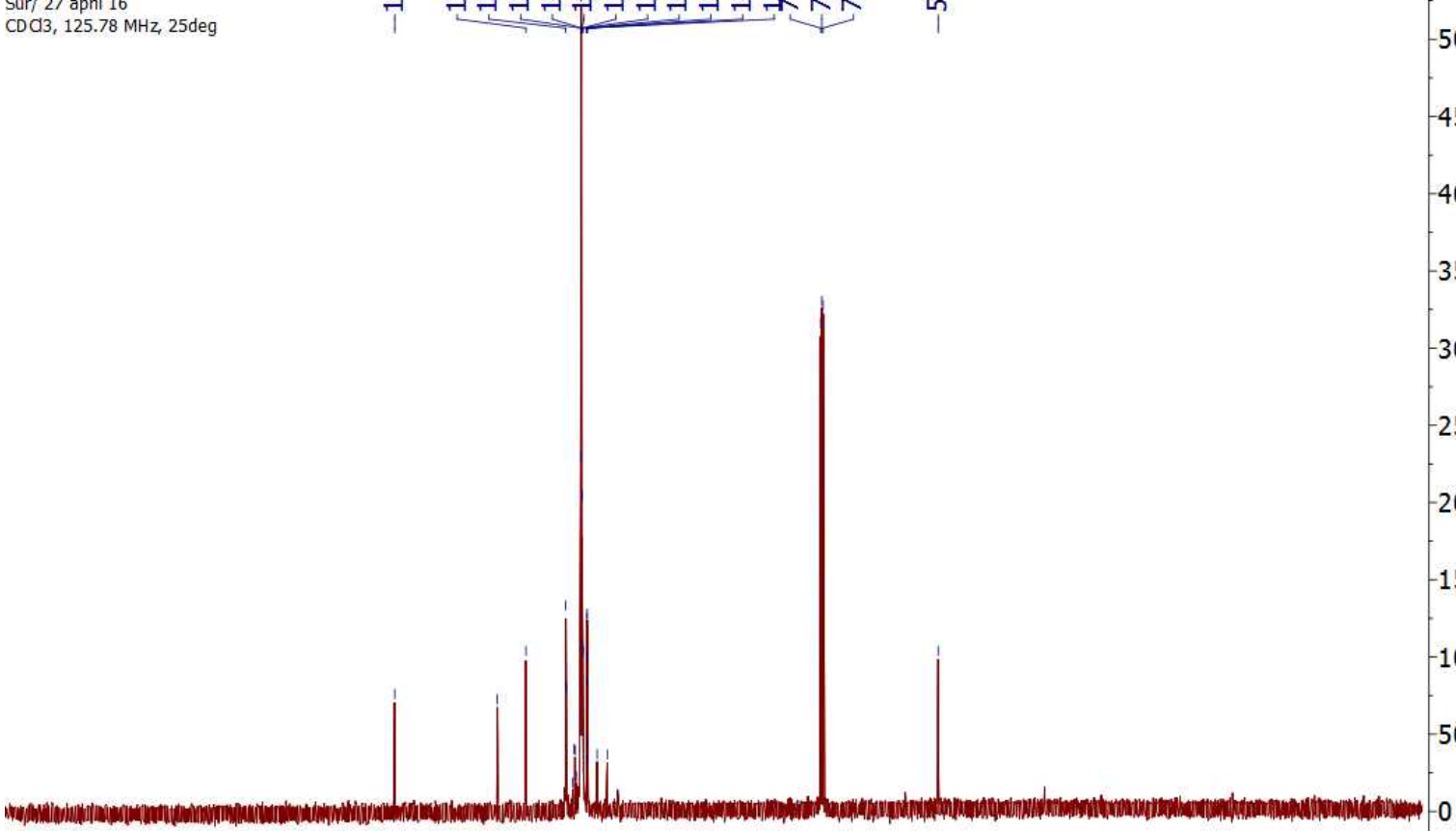


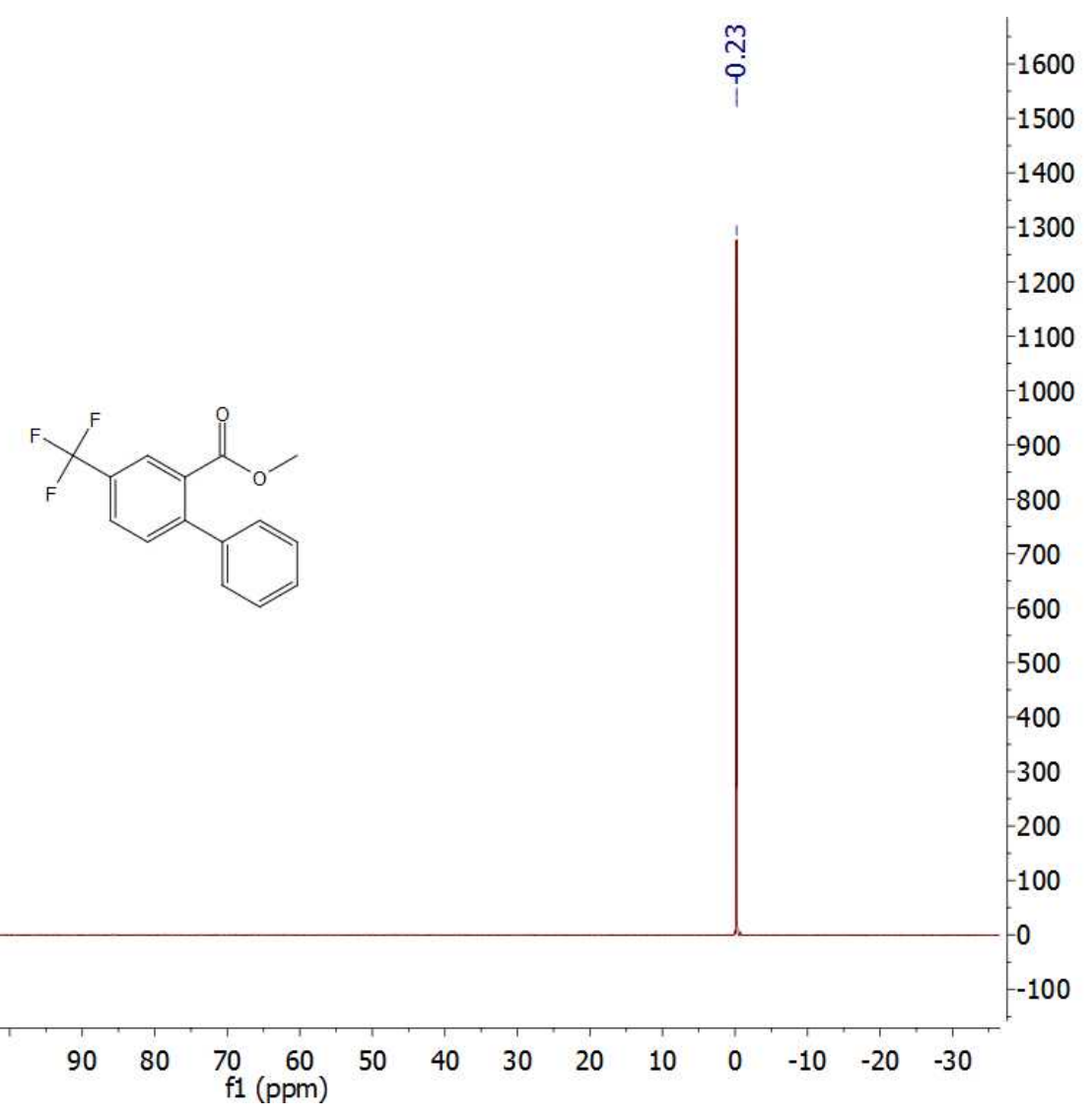




\section{4ca}

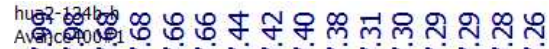

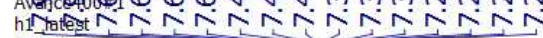
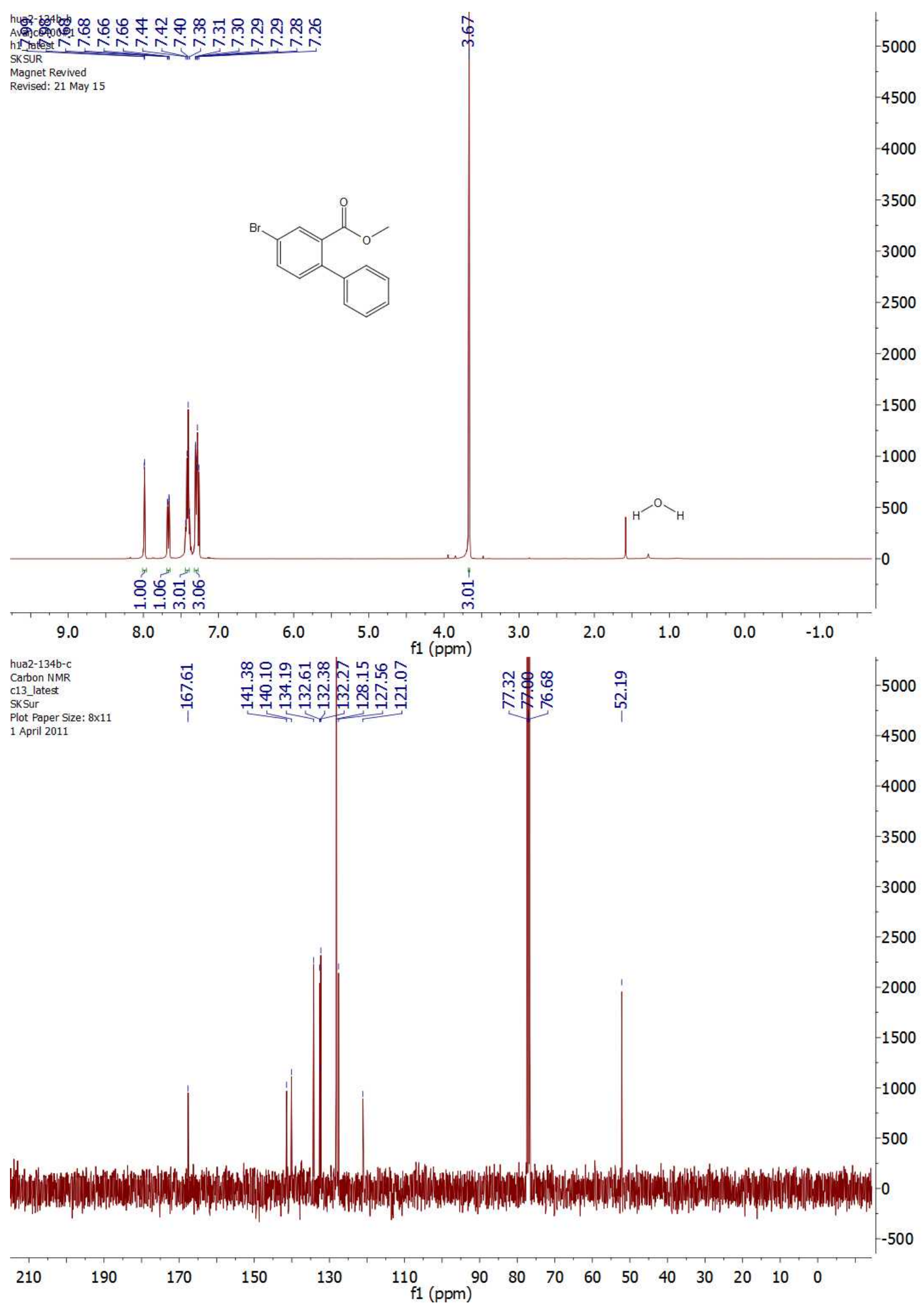


\section{4da}
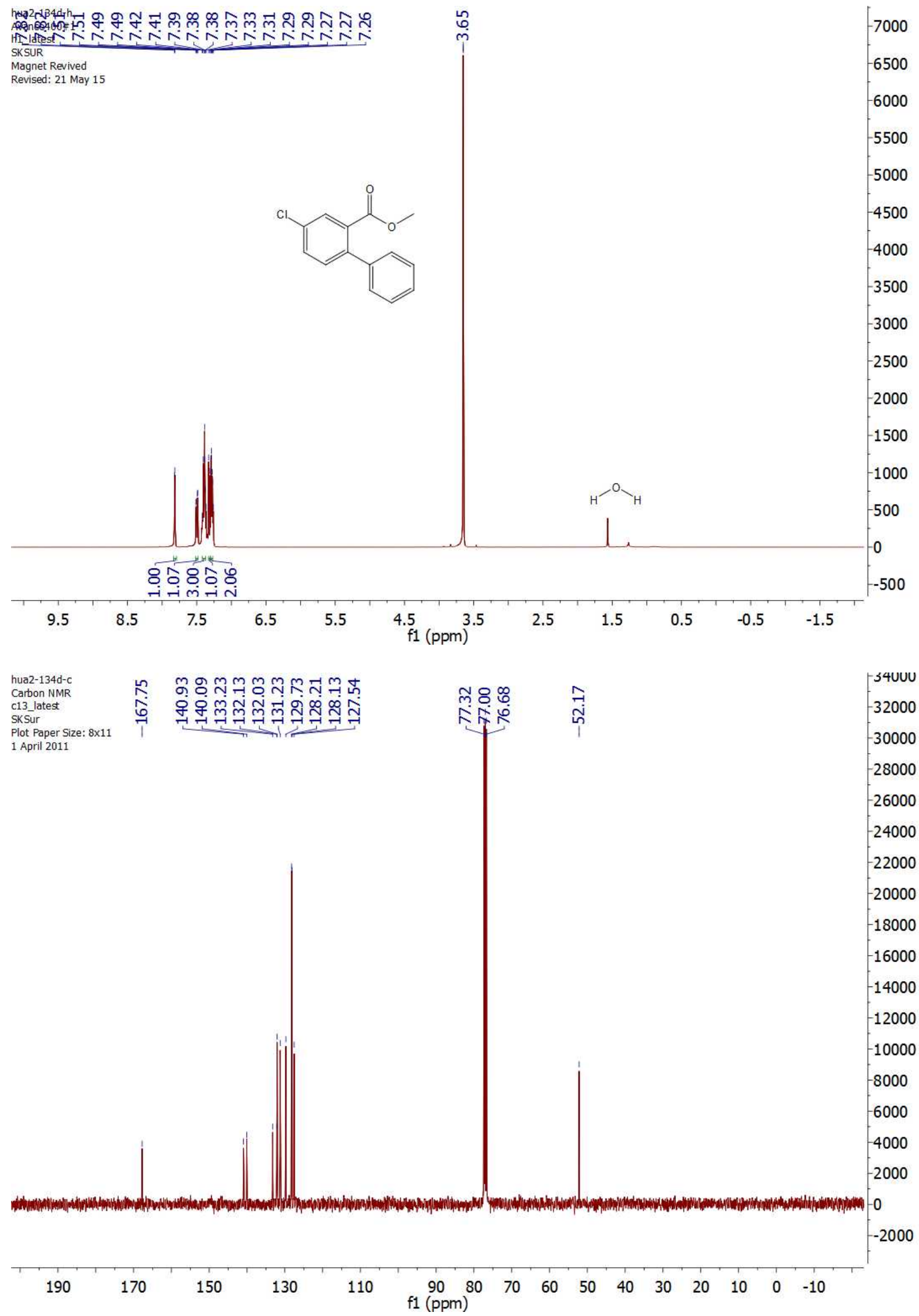


\section{4ea}

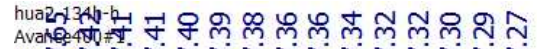

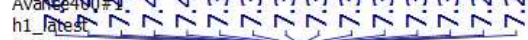

SKSUR

Magnet Revived

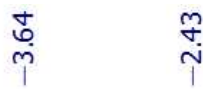

$-7500$

Revised: 21 May 15
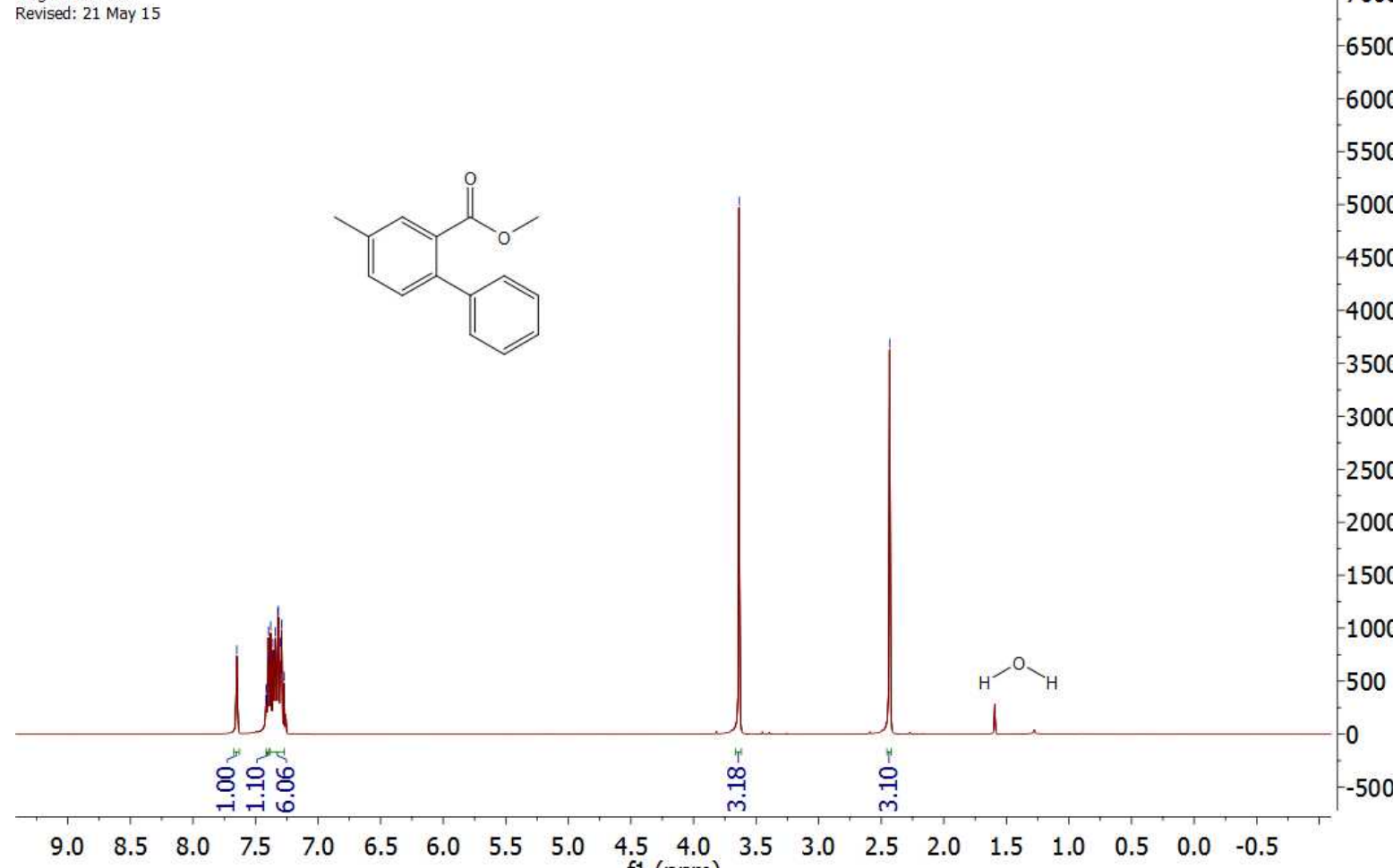

$\begin{array}{llllllllllllllllllll}9.0 & 8.5 & 8.0 & 7.5 & 7.0 & 6.5 & 6.0 & 5.5 & 5.0 & \underset{f 1}{4.5} & \underset{f(\mathrm{ppm})}{4.0} & 3.5 & 3.0 & 2.5 & 2.0 & 1.5 & 1.0 & 0.5 & 0.0 & -0.5\end{array}$

hua2-134h-c Carbon NMR

c13_latest

Plot Paper Size: $8 \times 11$

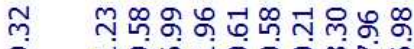

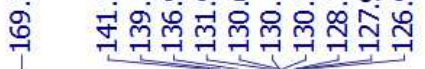

1 April 2011

\section{लำ}

송

$\stackrel{\text { मे }}{\text { मे }}$

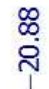


4fa

hua2-134k-h
Avance400\#1
h1_latest

h1_latest

Magnet Revived

$\infty \infty \infty \infty \infty \infty N)$

Revised: 21 May 15

$\begin{array}{ll}\stackrel{0}{0} & \stackrel{0}{0} \\ \stackrel{0}{0} & \stackrel{i}{1}\end{array}$

8000
-7500
7000

7000

$-6500$

$-6000$

$-5500$

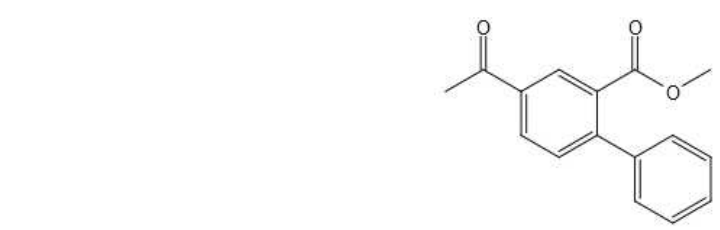

5000

4500

4000

3500

3000

2500

2000

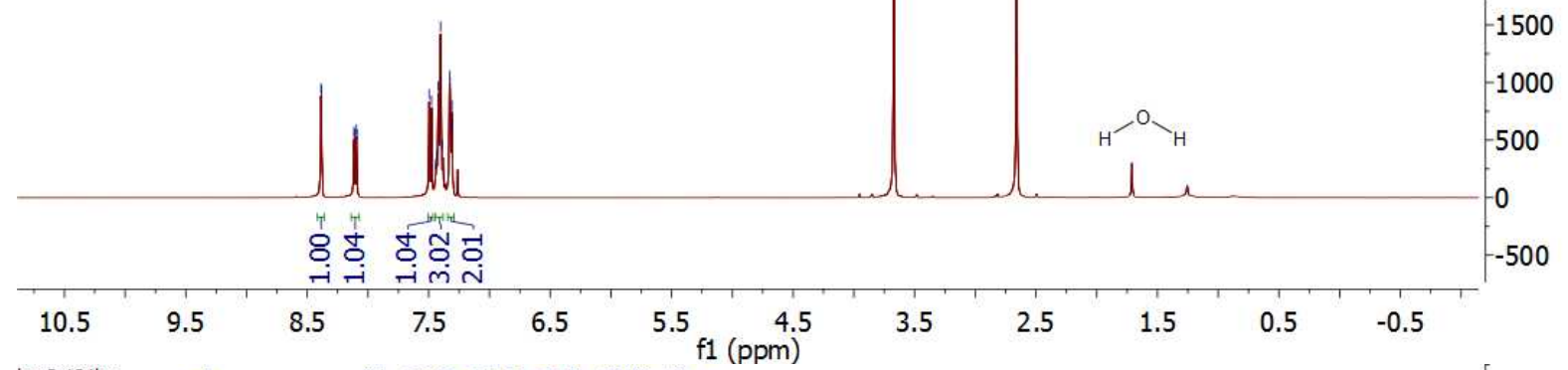

10.5

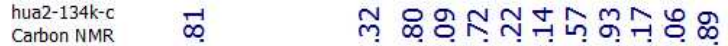

c13_latest

SkSur

1 April 2011

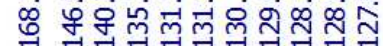

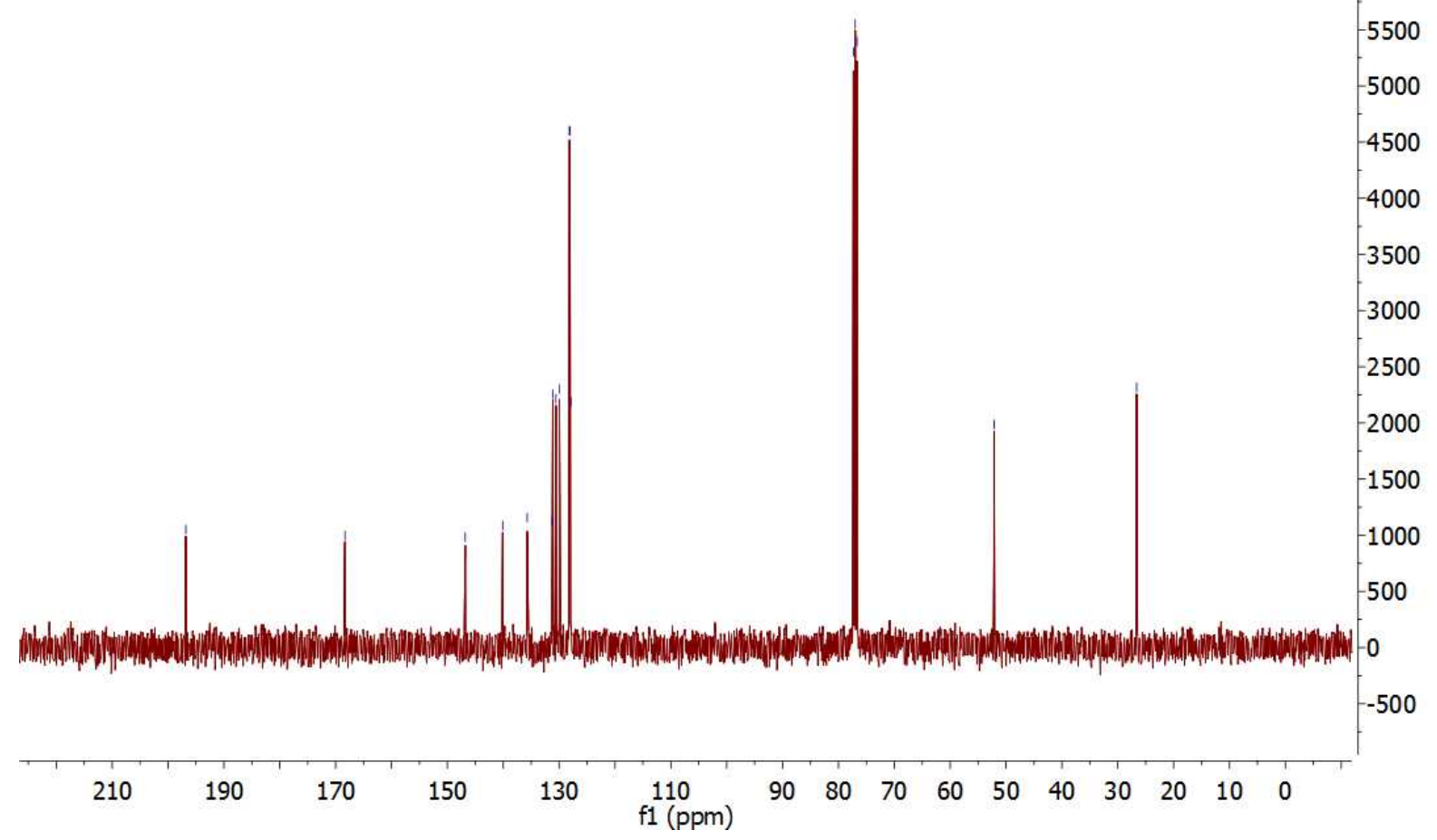




\section{4ga}

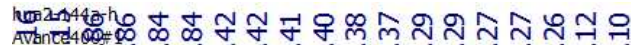

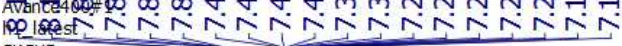
SKSUR

Magnet Revived

Revised: 21 May 15

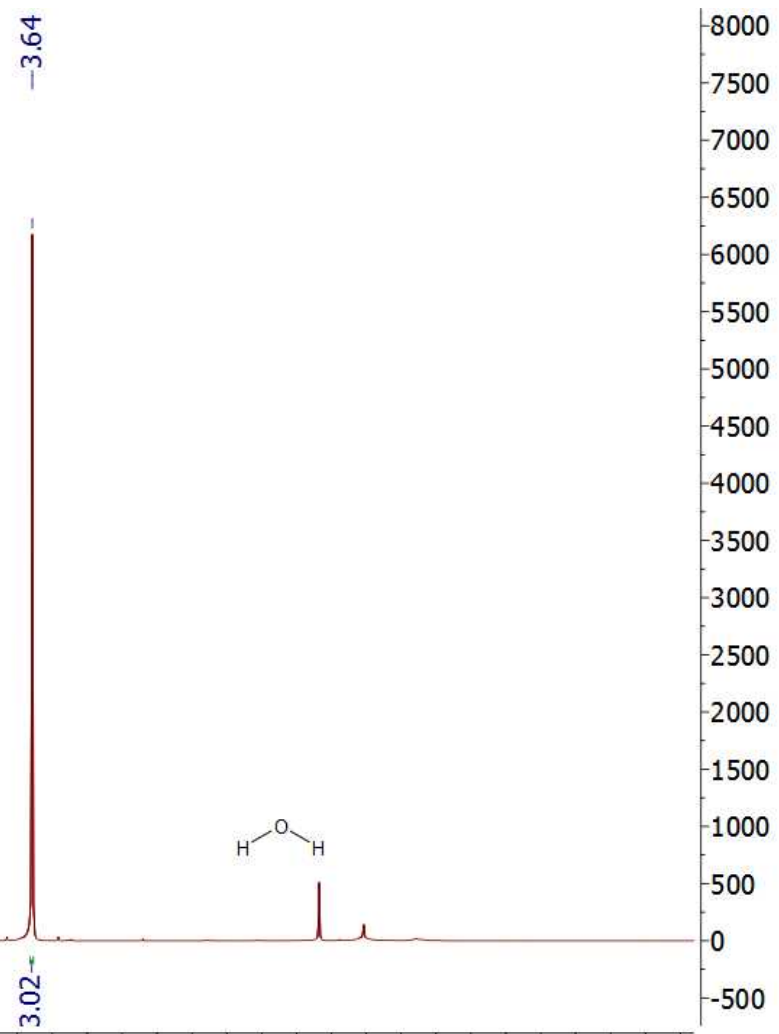

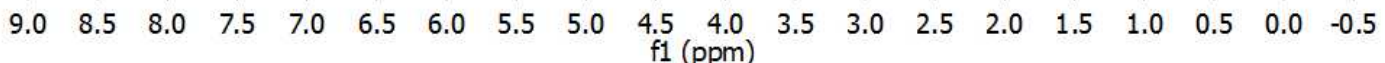

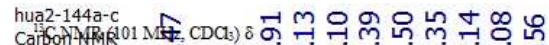

SKSut
Plot Paper Size: 8x11

1 April 2011

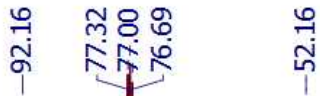

$-8000$

$-7500$

$-7000$

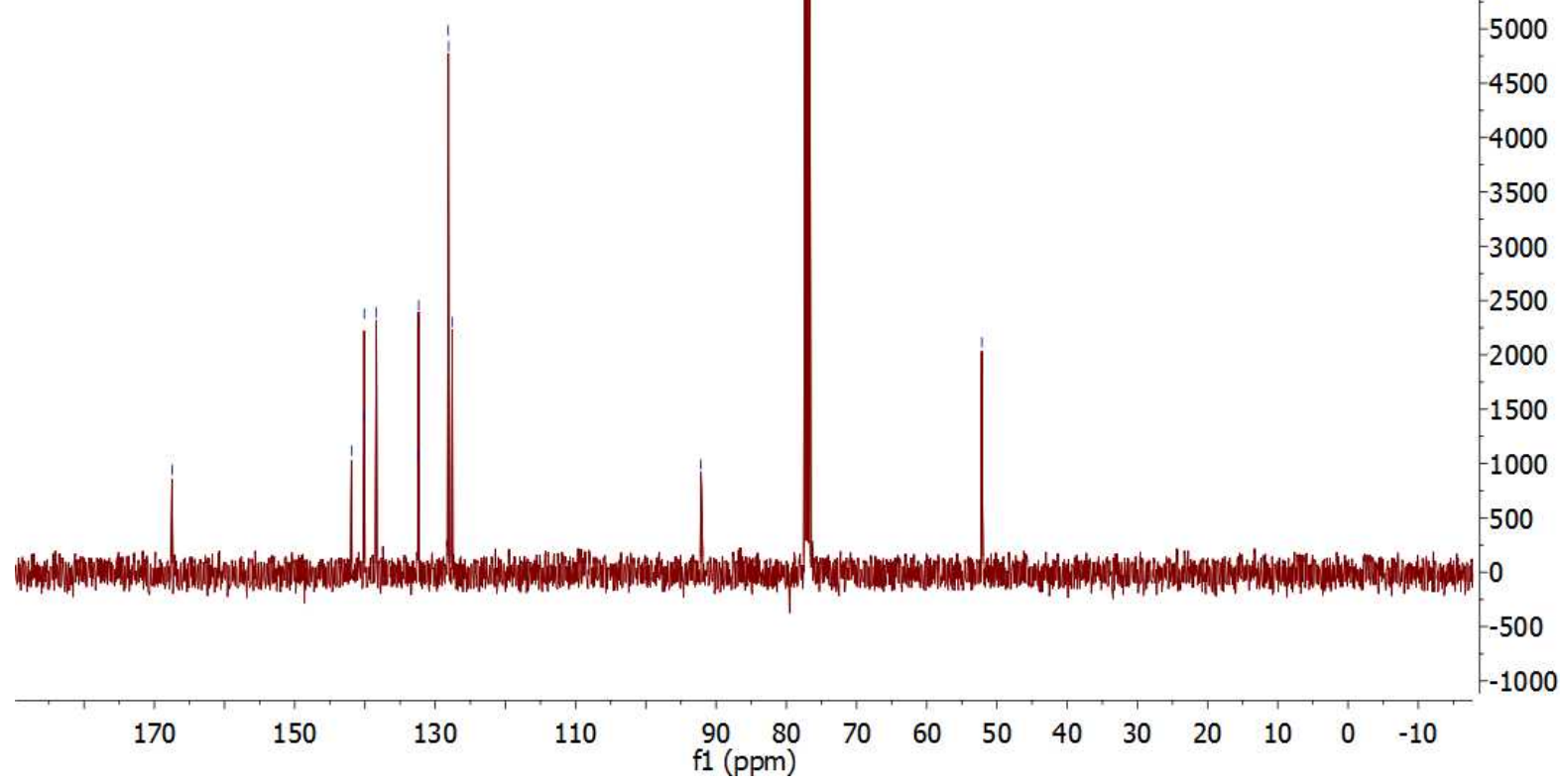




\section{4ha}

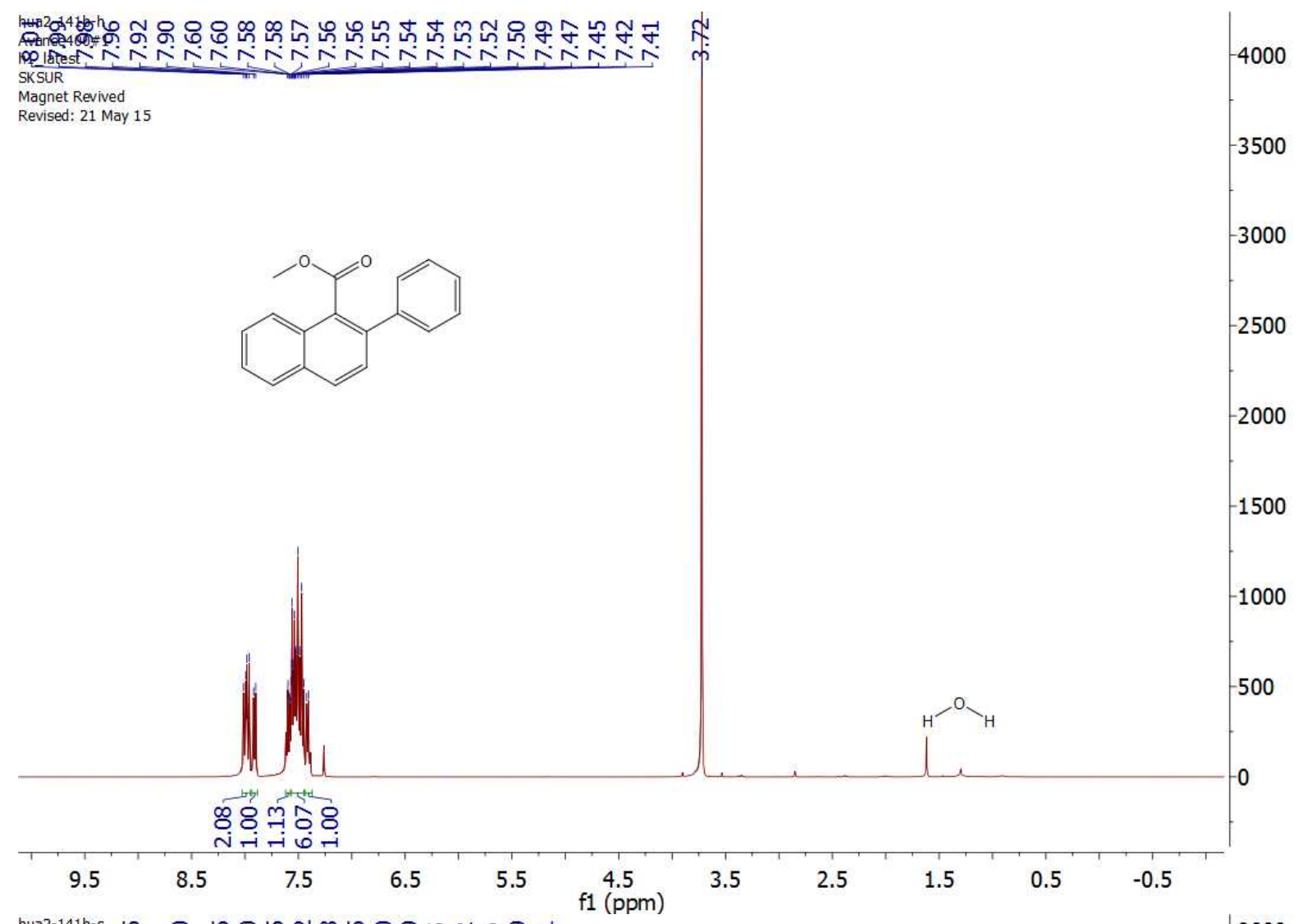

huaz-141-c

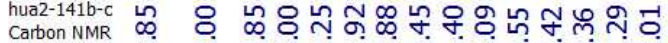
c13/lates

SKSur Slot Paper Size: $8 \times 11$

1 April 2011

ํㅗ용

路

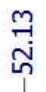

9000

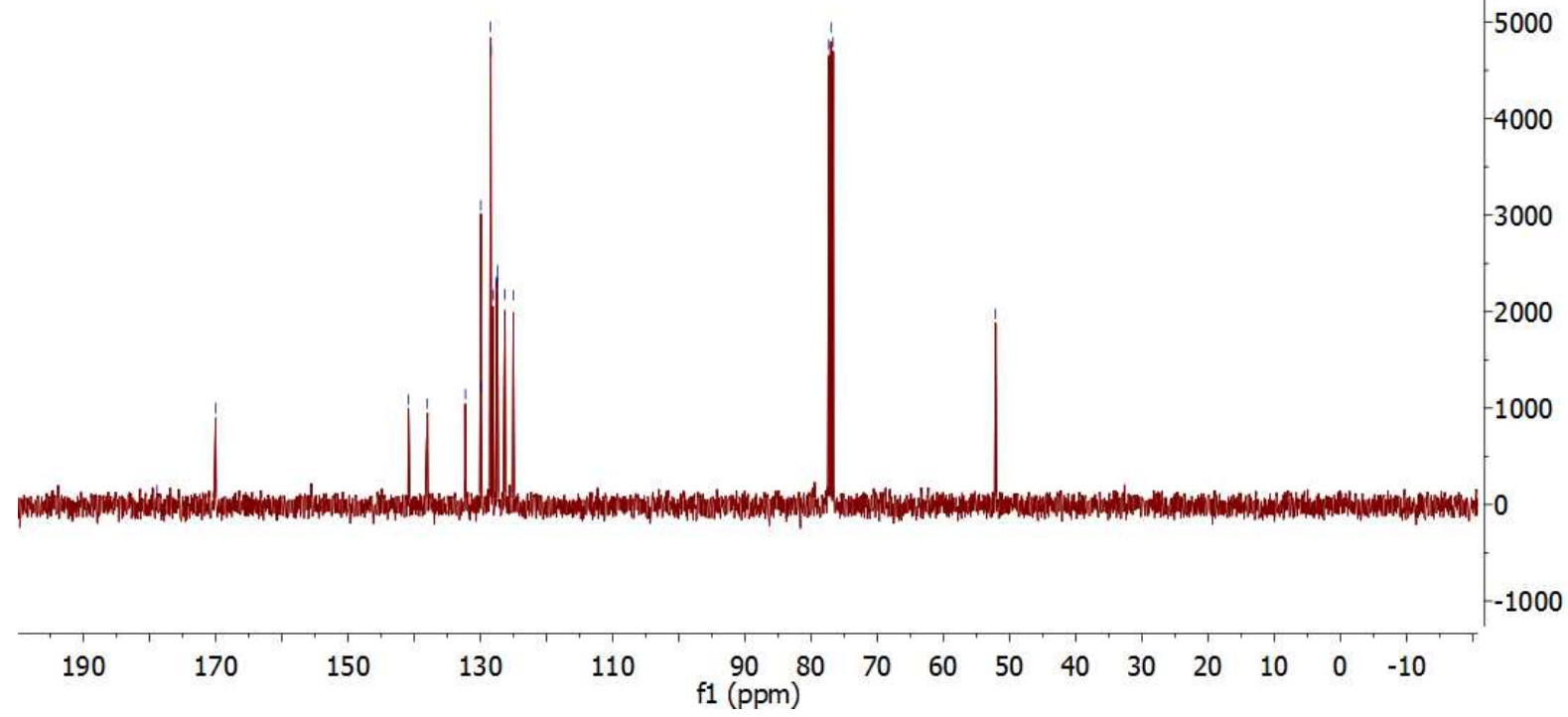


4ia

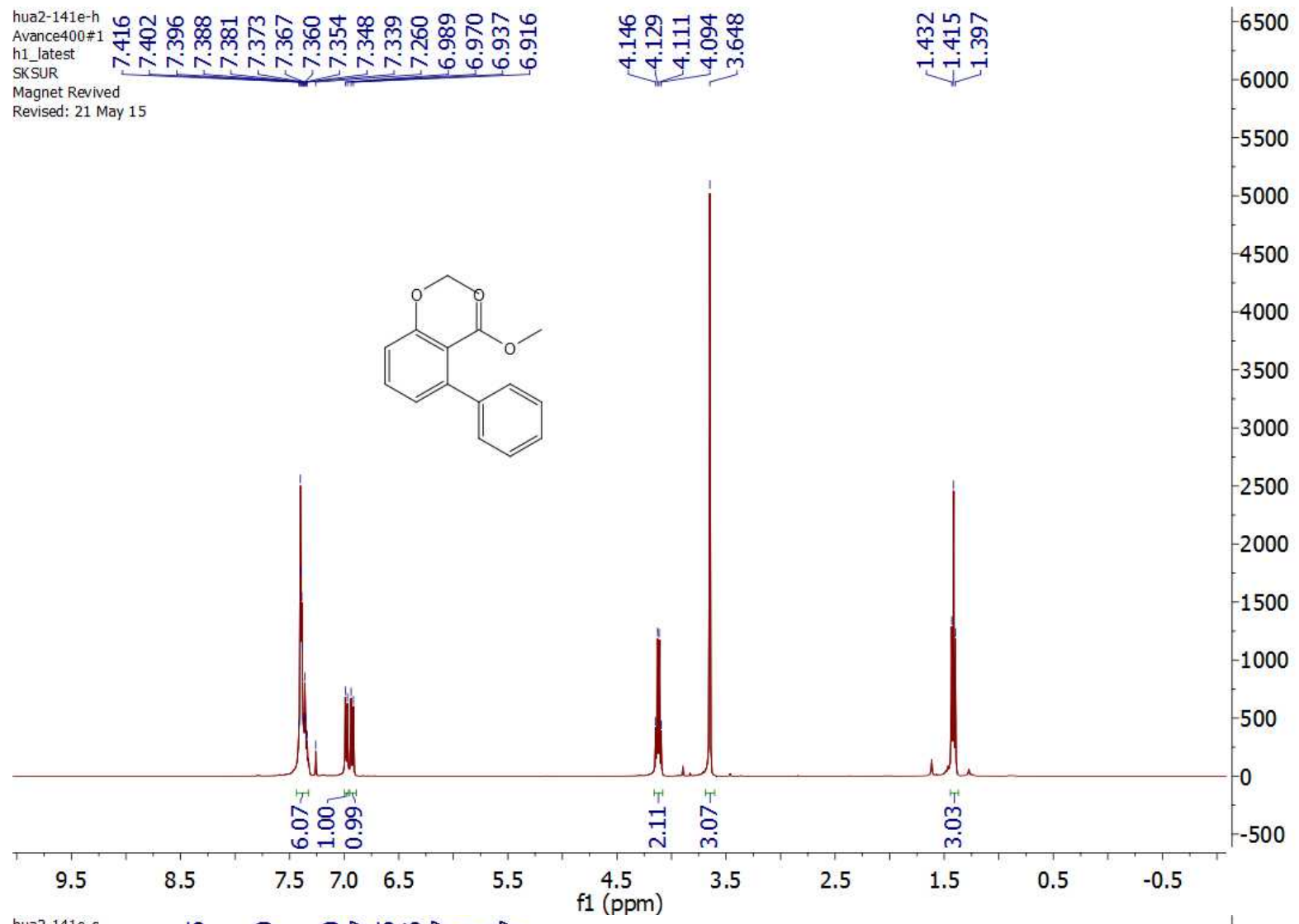

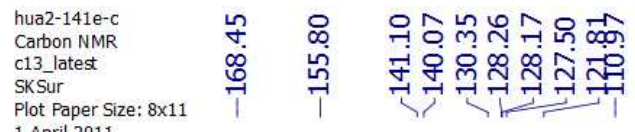

1 April 2011

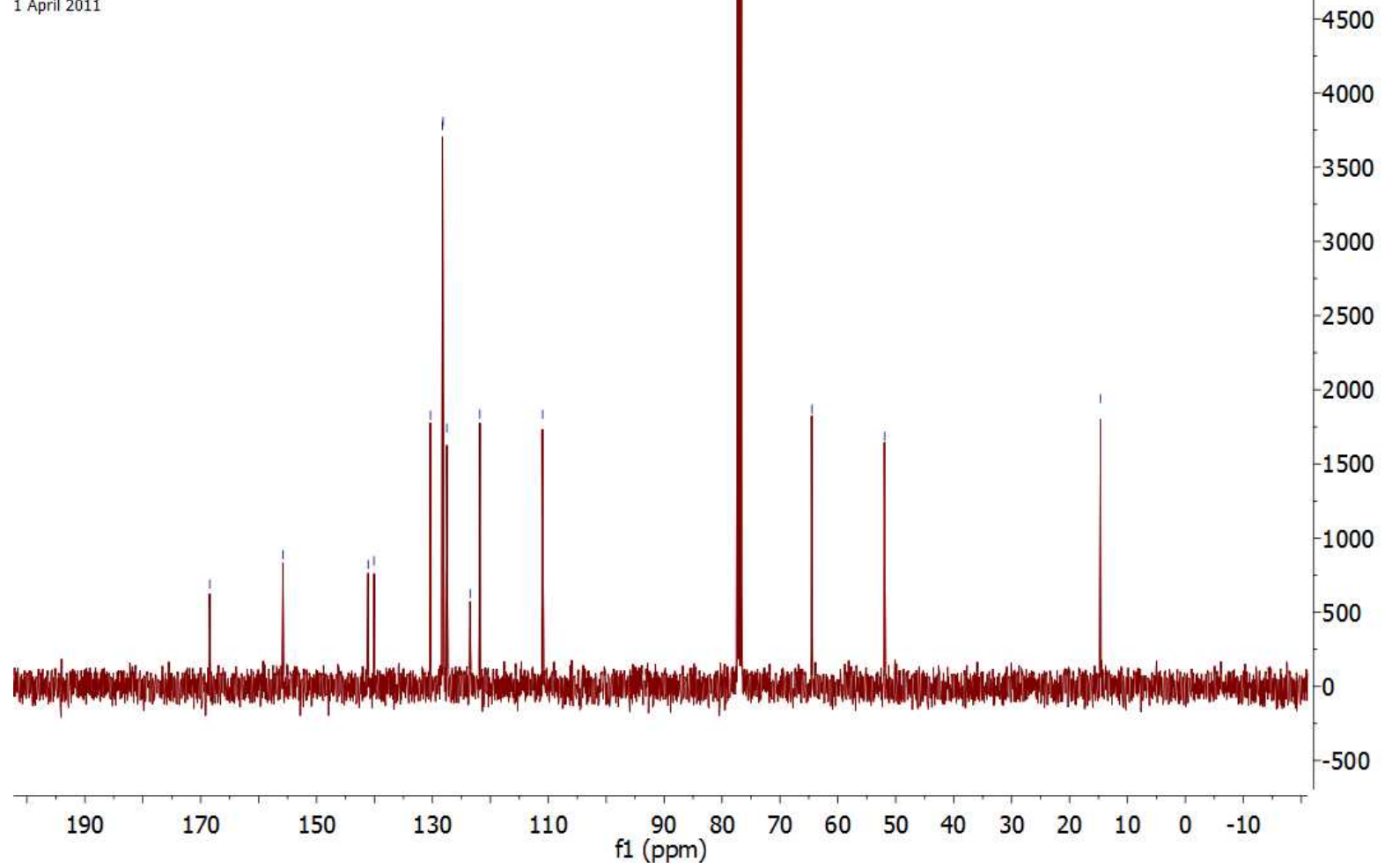




\section{$4 j a$}

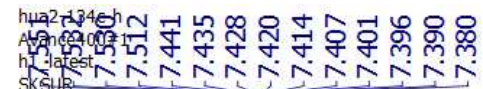

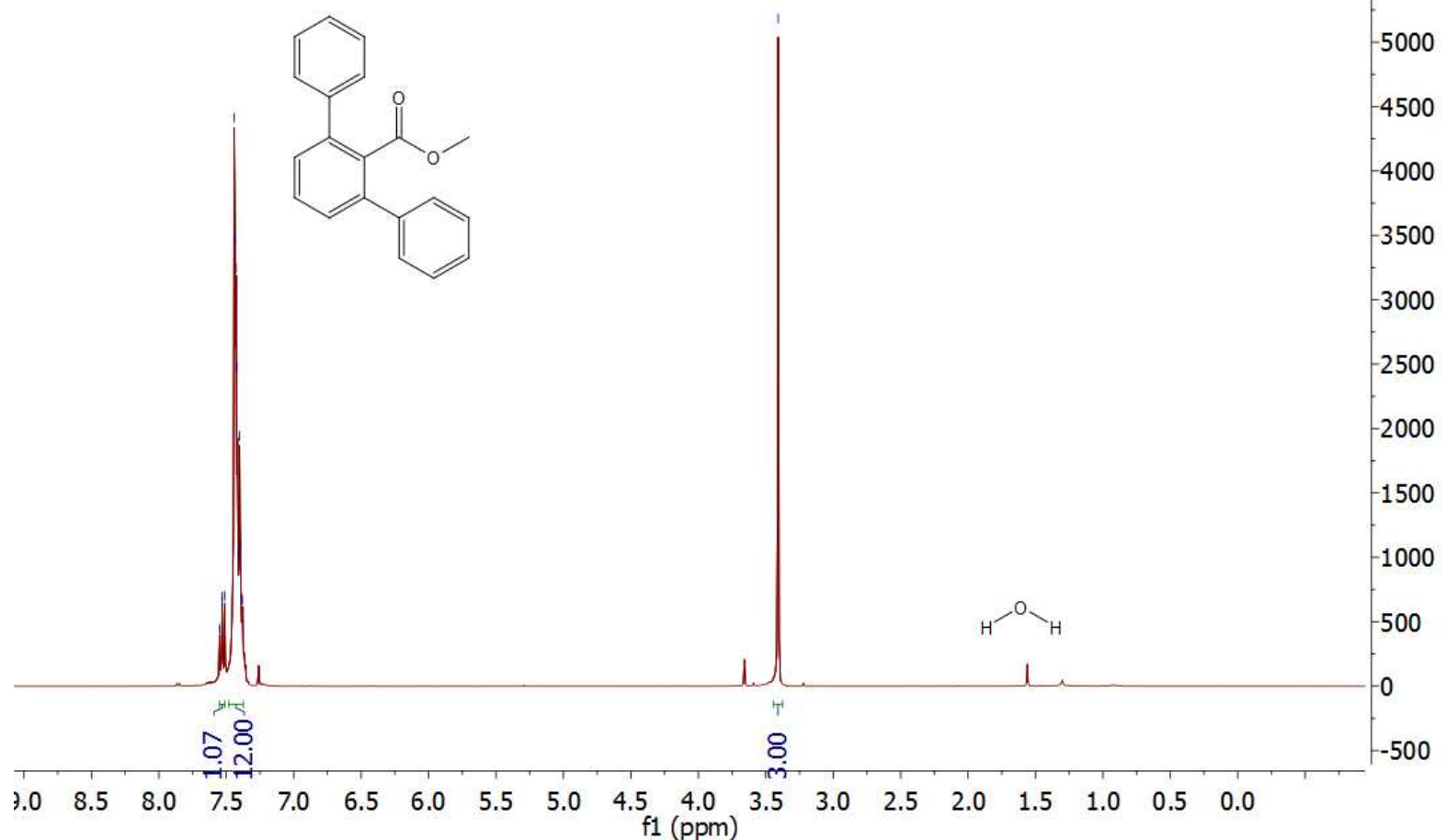

hua2-134c-c o

carbon NMR Iatest

PKSur Paper Size: $18 \times 11$

1 April 2011

fㅇำ लिल

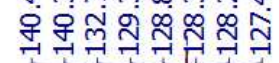

ำ 용

쇼욘

กิ

9000

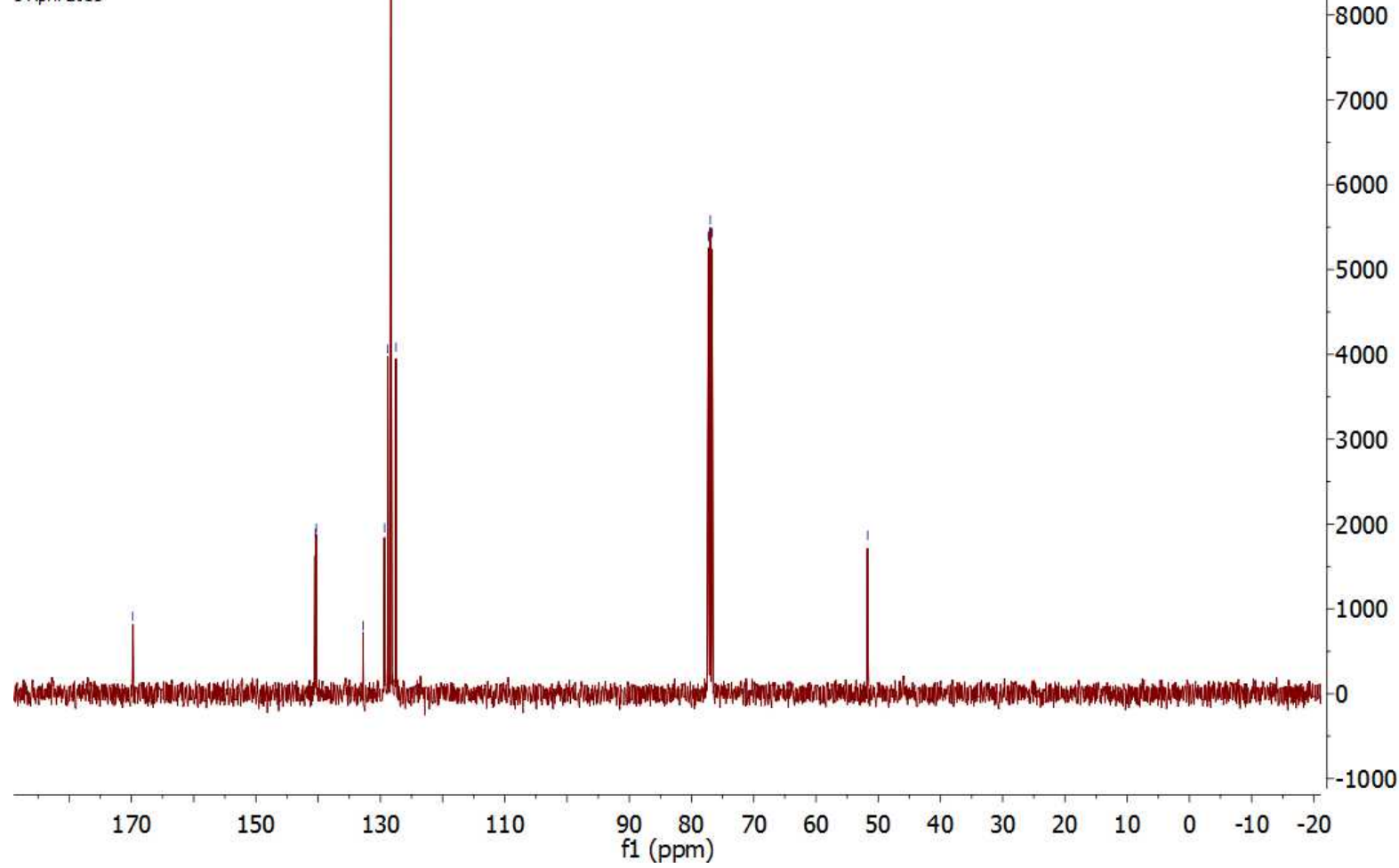


4ka

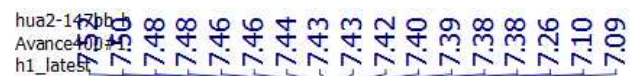

SKSUR

Magnet Revived

Revised: 21 May 15

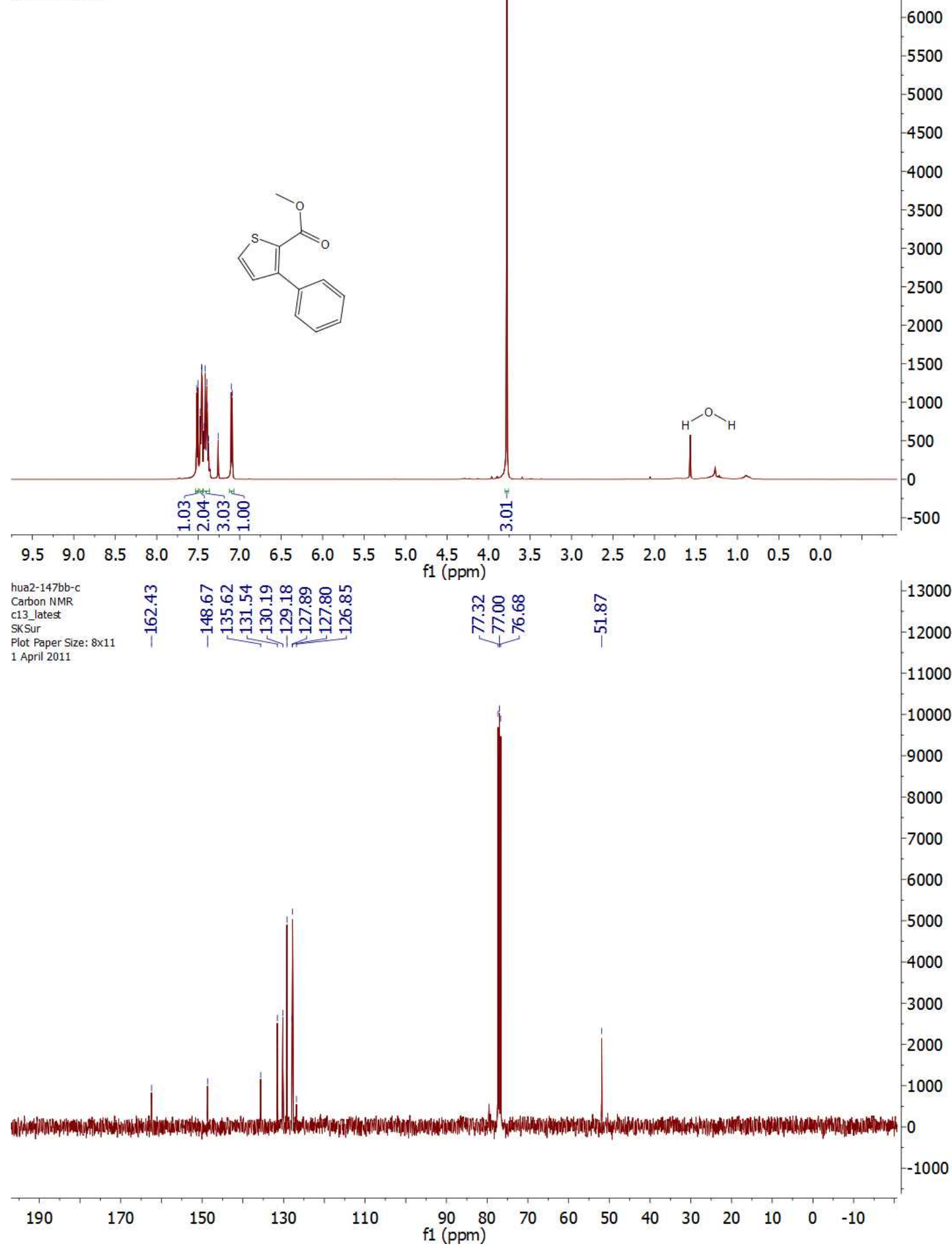




\section{$4 k t$}

hua2-156c-h

Avancete001
h1 latest

SKSUR

Magnet Revived

Revised: 21 May 15
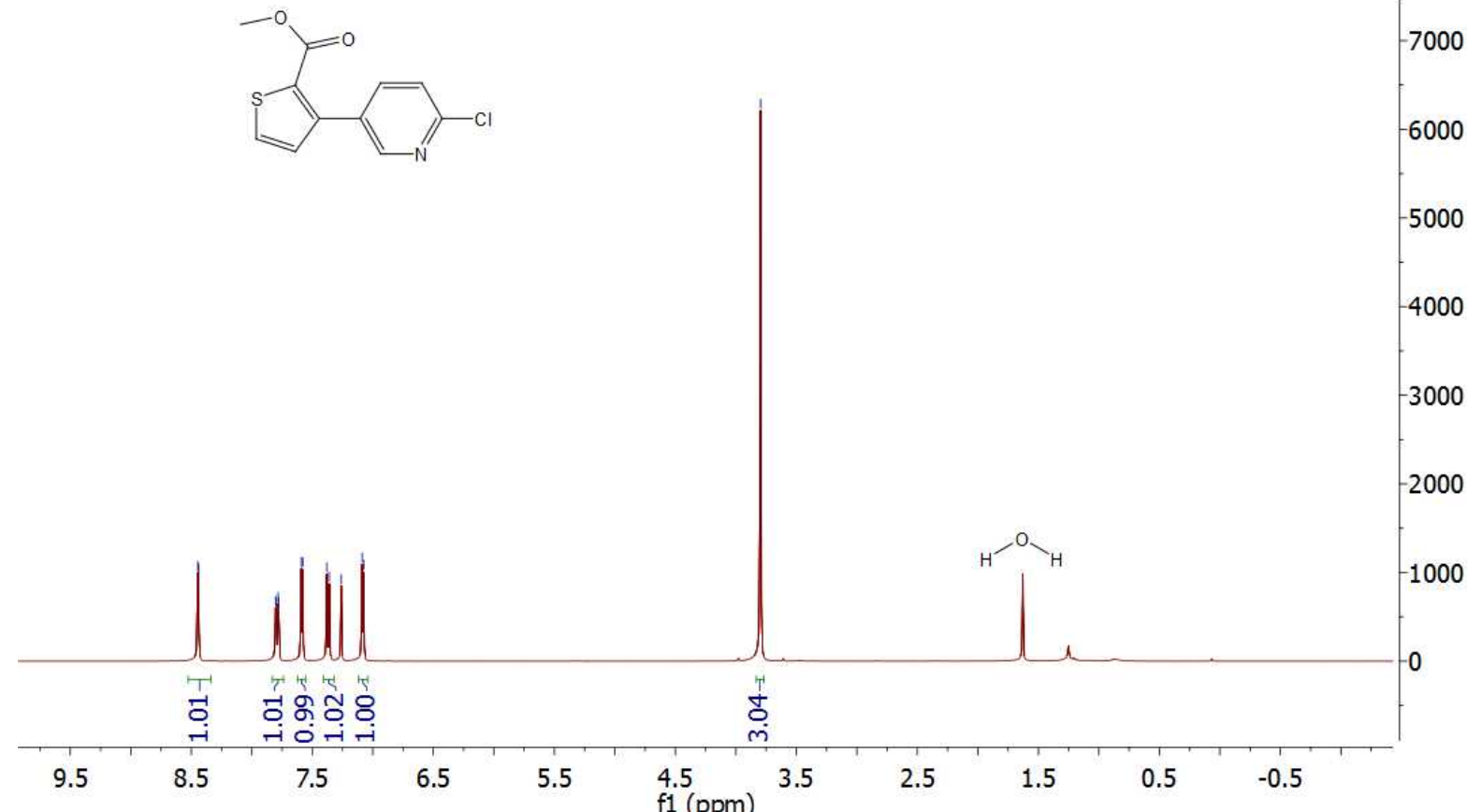

hua2-156c-C

Carbon NMR

C13_latest

Plot Paper Size: $8 \times 11$

뭉 국

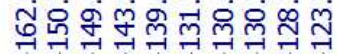

f1 (ppm)

1 April 2011

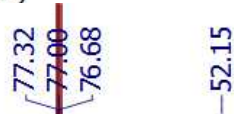

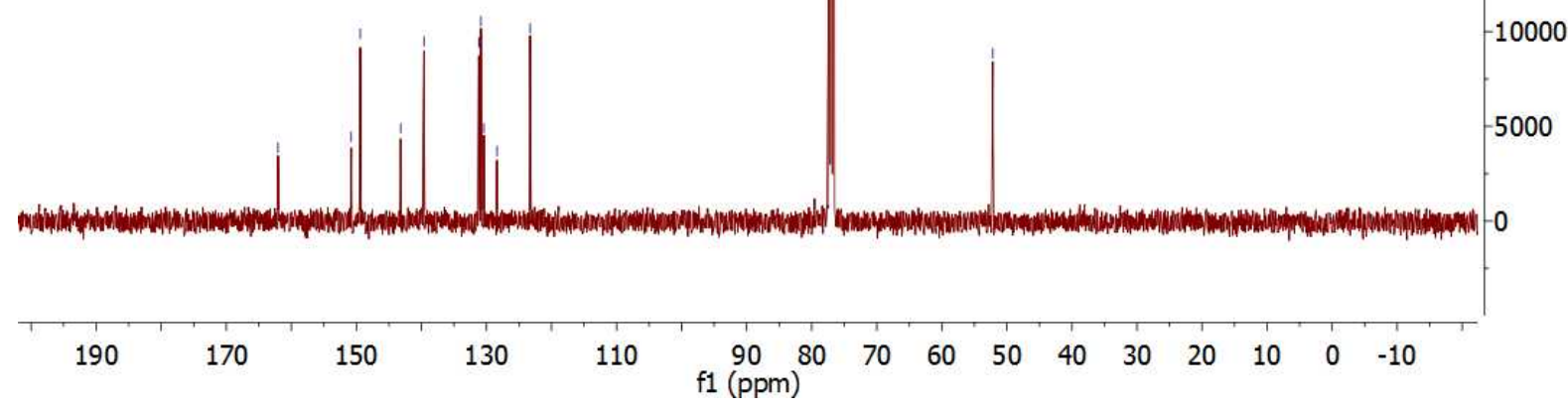


$41 a$

hua2-168d-h-1

Avance400\#

SKSUR

m

$-3800$

Magnet Revived

Revised: 21 May 15

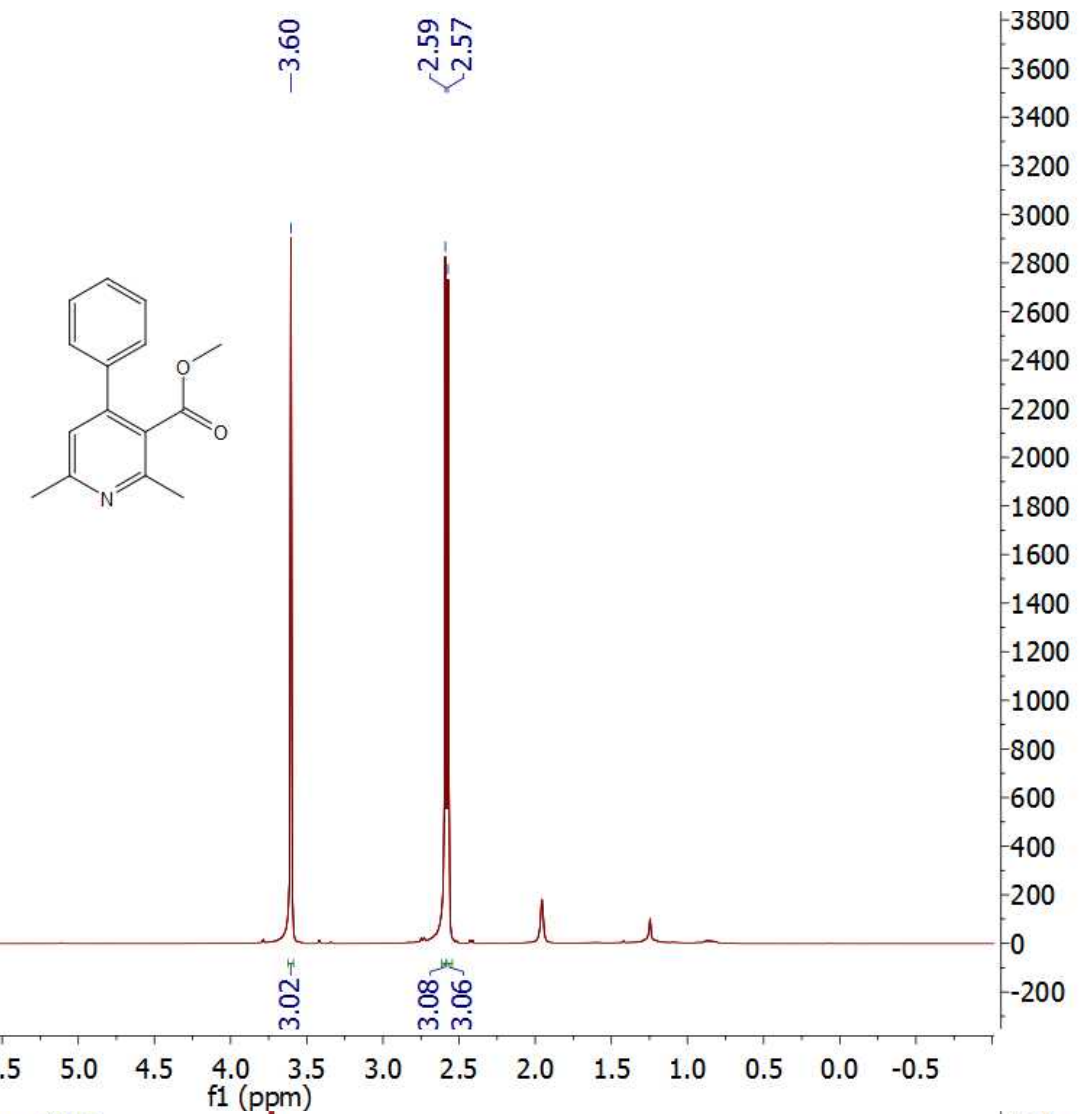

$\begin{array}{llllllllll}8.5 & 8.0 & 7.5 & 7.0 & 6.5 & 6.0 & 5.5 & 5.0 & 4.5 & 4.0 \\ \mathrm{f1}(\mathrm{ppm})\end{array}$

hua2-168d-c

Carbon NMR

c13_lates

Plot Paper Size: $8 \times 11$

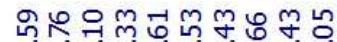

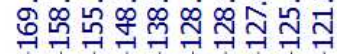

ल $\$$

你它 ก

700

1 April 2011

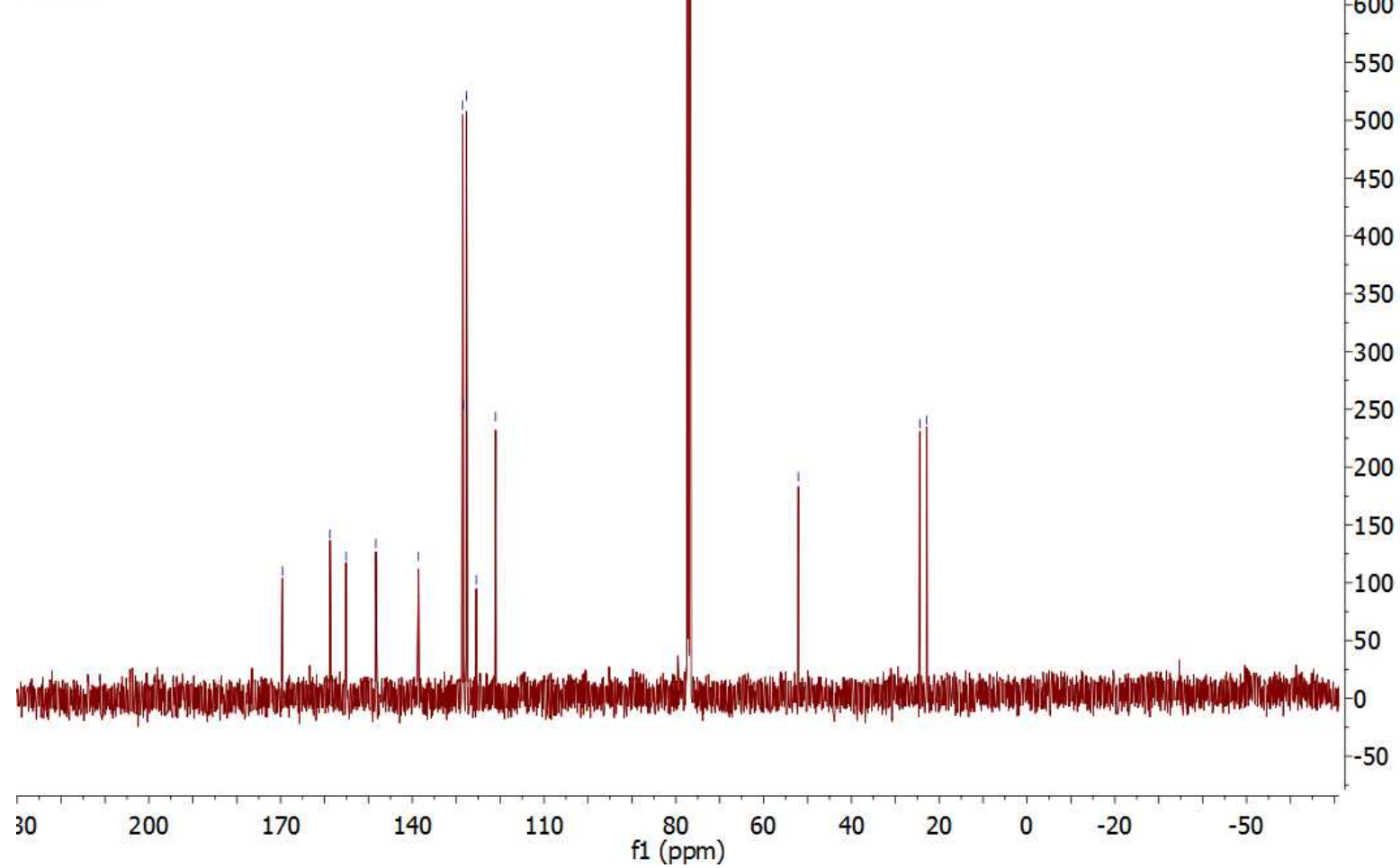




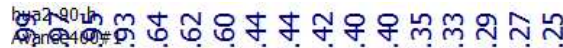
(1) SKSUR

Magnet Revived

Revised: 21 May 15
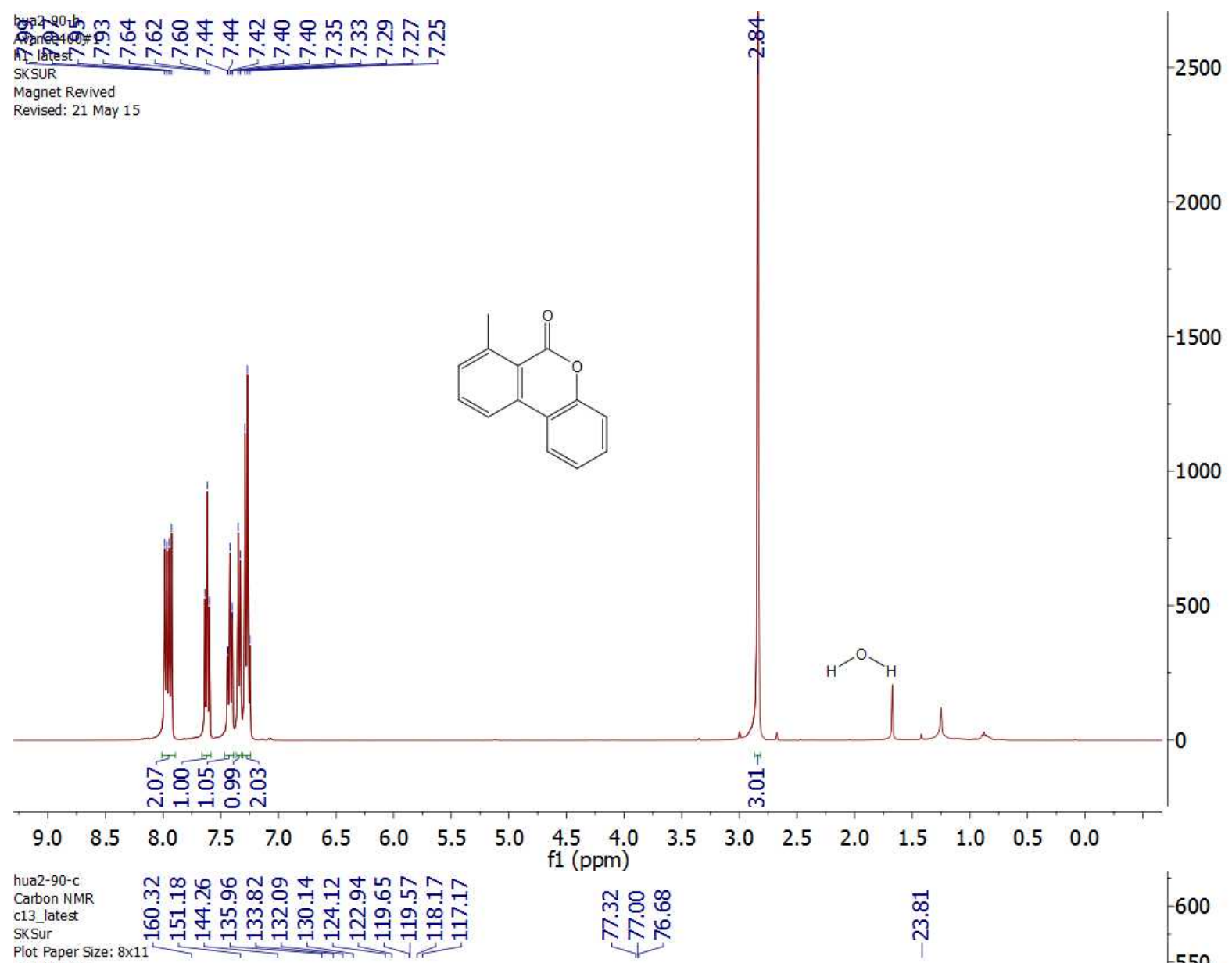

ํㅛㅇㅛ

슌

ஸे

$-600$

Plot Paper Size: 8x11

1 April 2011

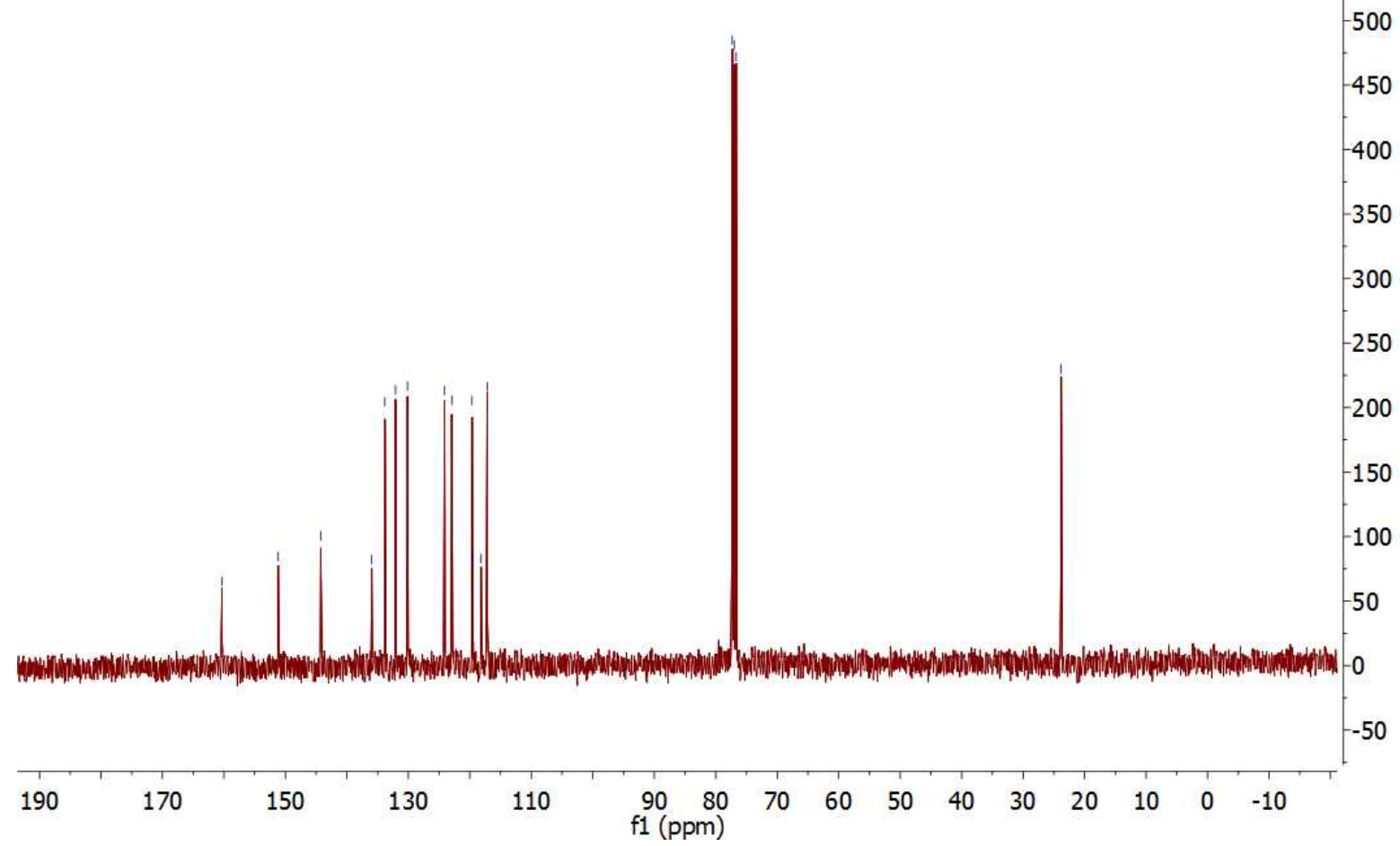




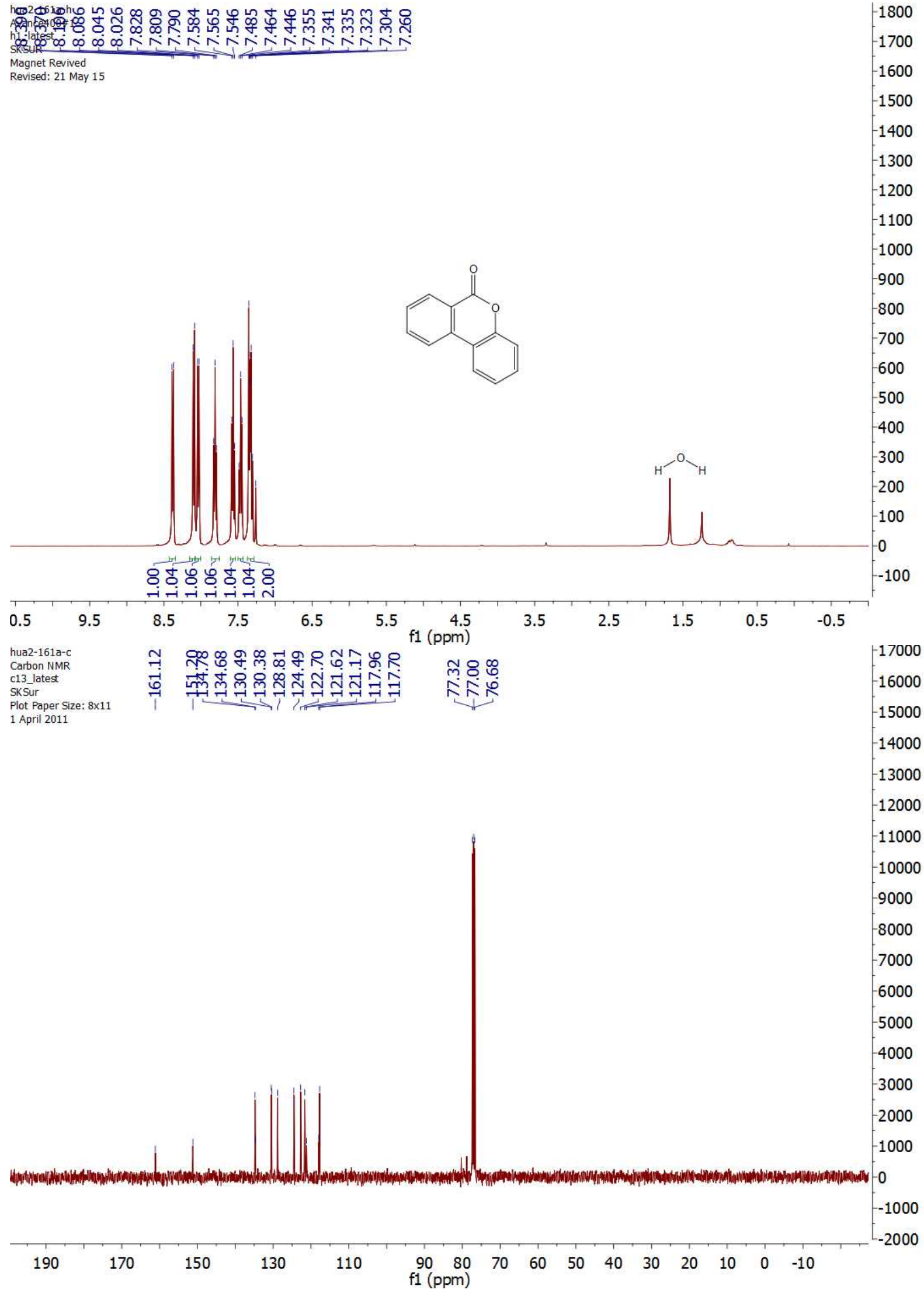


6c

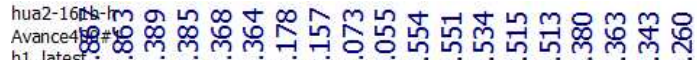

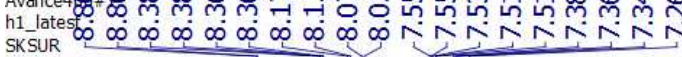

Magnet Revived

Revised: 21 May 15

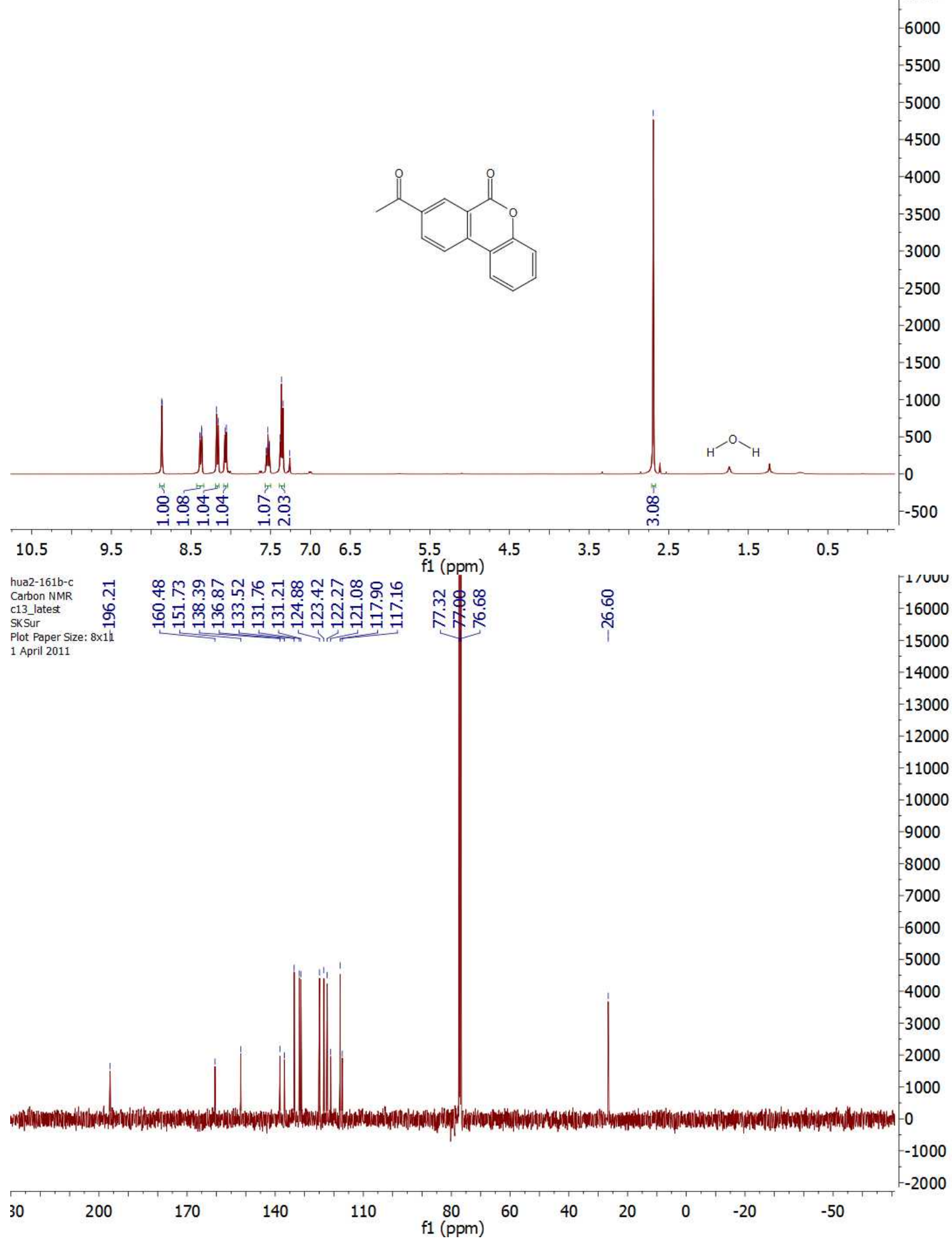



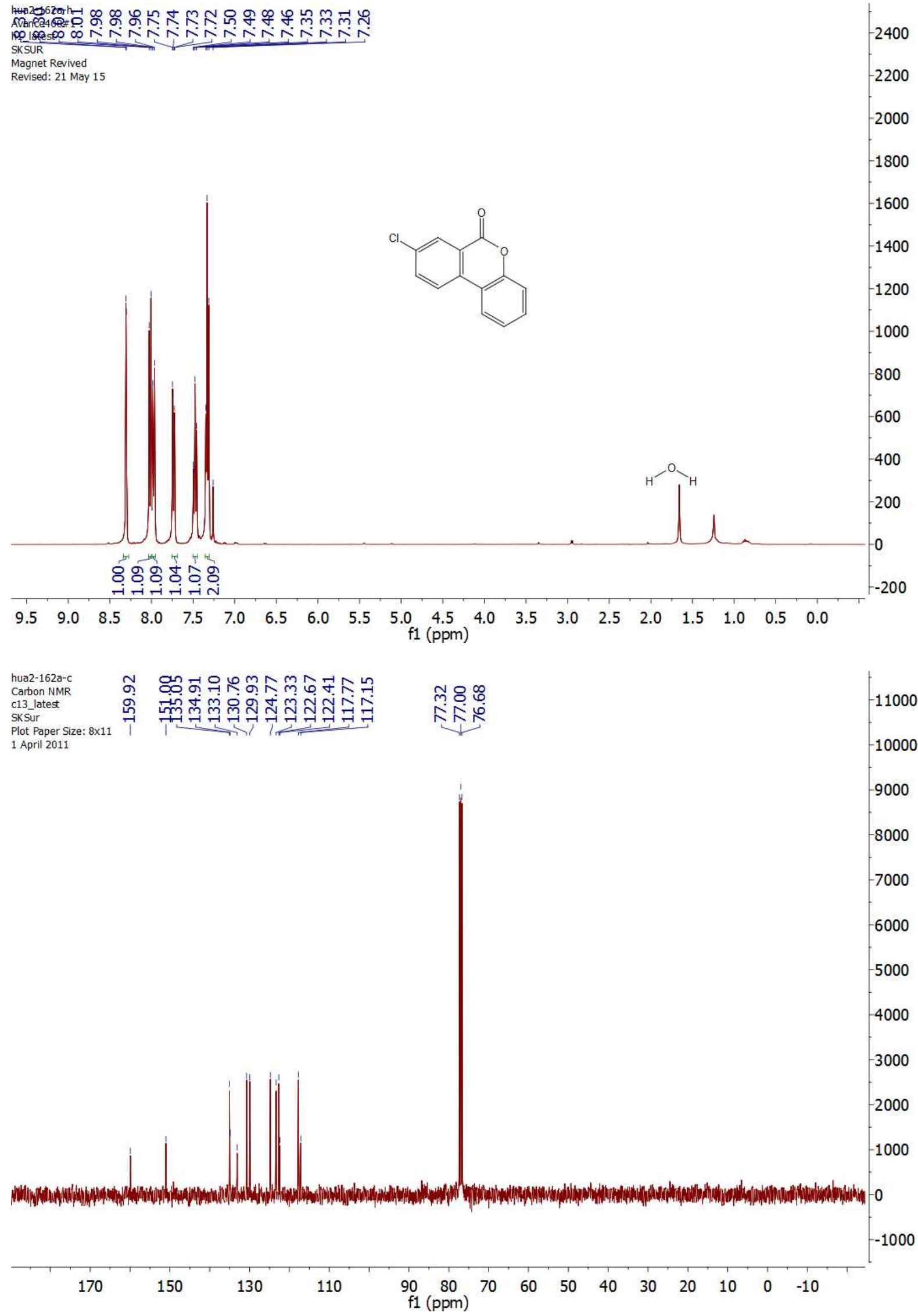
hua2-162b-tm 규

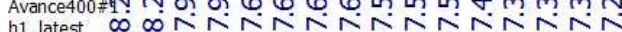

SKSUR

Magnet Revived

Revised: 21 May 15

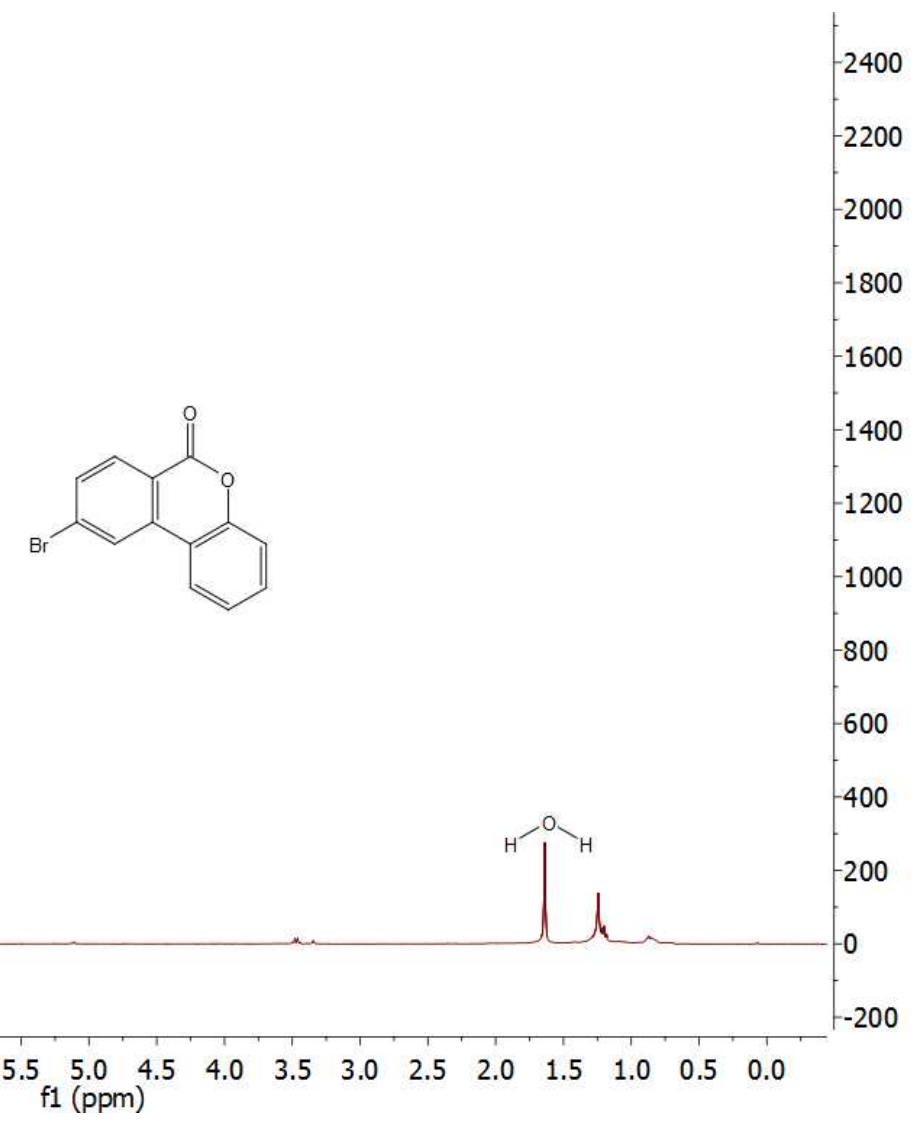

hua2-162b-c Carbon NMR c13_latest

SKSur
Plot Paper Size: $8 \times 11$

บ. ำกㄴำ

०.

꾜윰

슈요

$-45000$

1 April 2011

वृ.

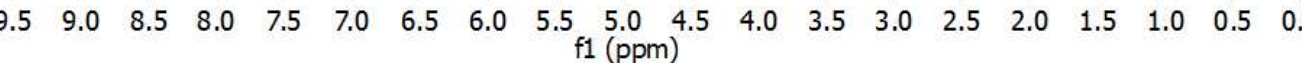

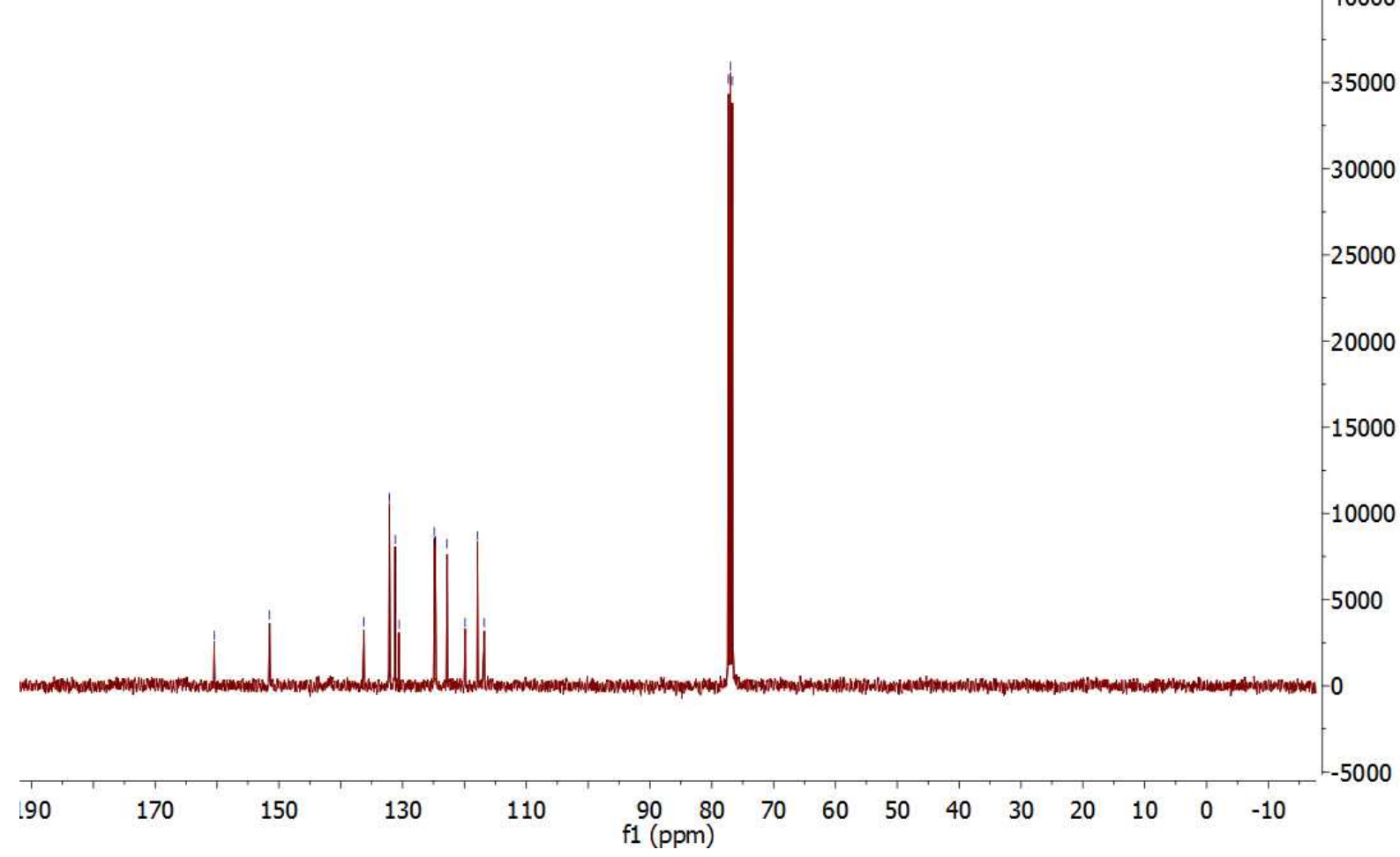


6f

hua2-162-hn
Avance400*1

Avanceteon

SKSUR

Magnet Revived

Revised: 21 May 15

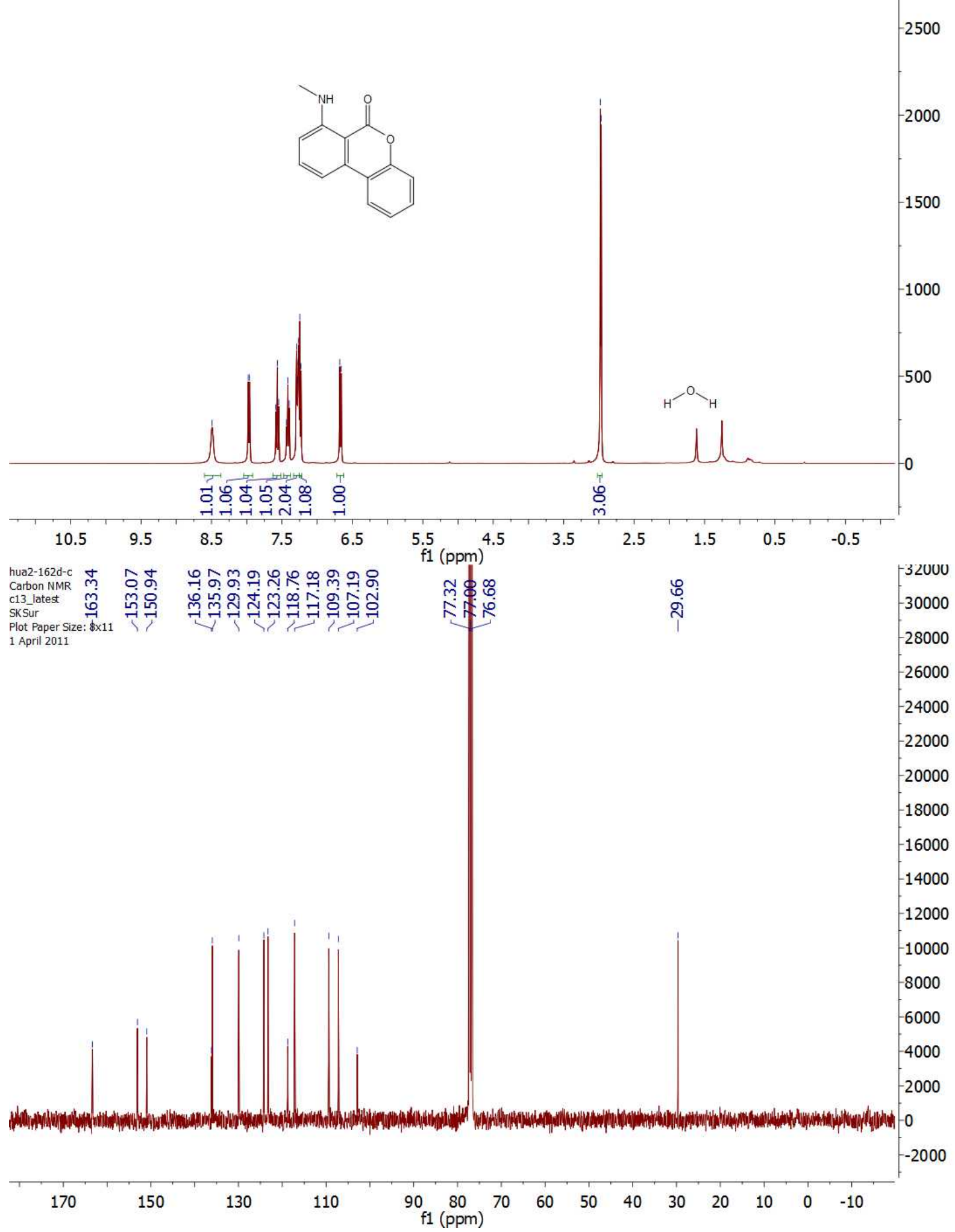


6ga

hua2-6g-h

Avance $400 \# 1$

h1_latest

Magnet Revived

Revised: 21 May 15

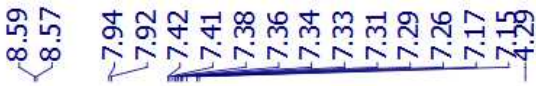

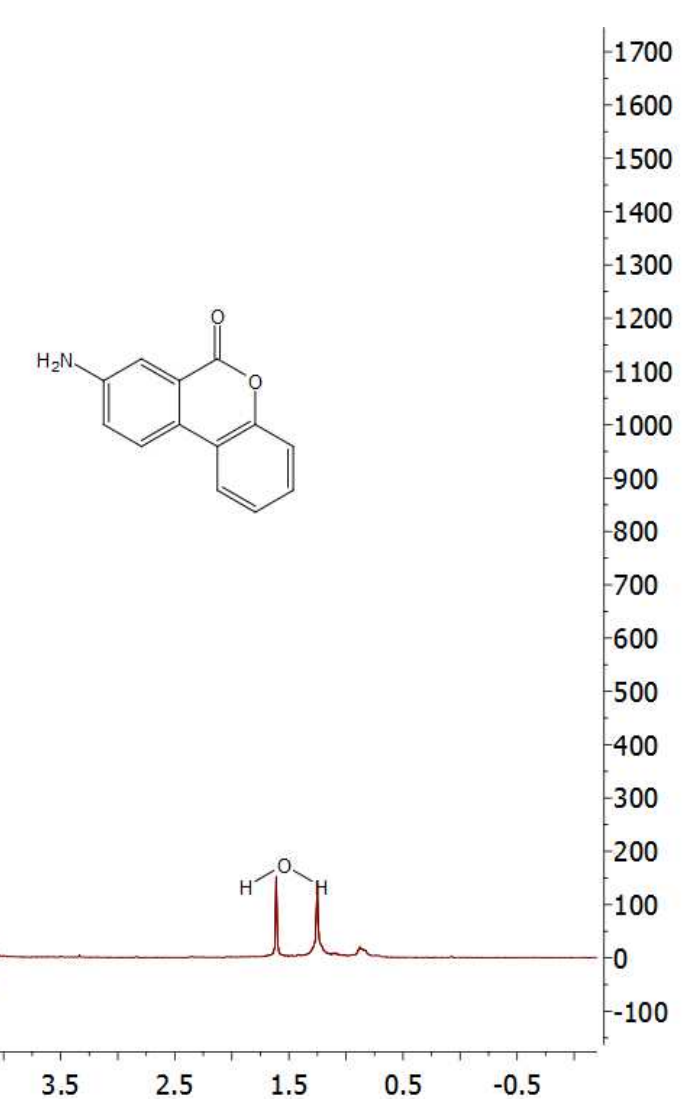

$\begin{array}{llllllllllllll}11.5 & 10.5 & 9.5 & 8.5 & 7.5 & 6.5 & 5.5 & 4.5 & 3.5 & 2.5 & 1.5 & 0.5 & -0.5\end{array}$

hua2-165b-c

Carbon NMR

SKSur

Plot Paper Size: $8 \times 11$

1 April 2011

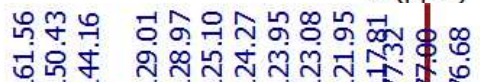

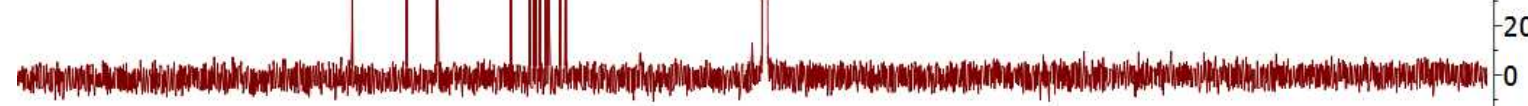


6ha
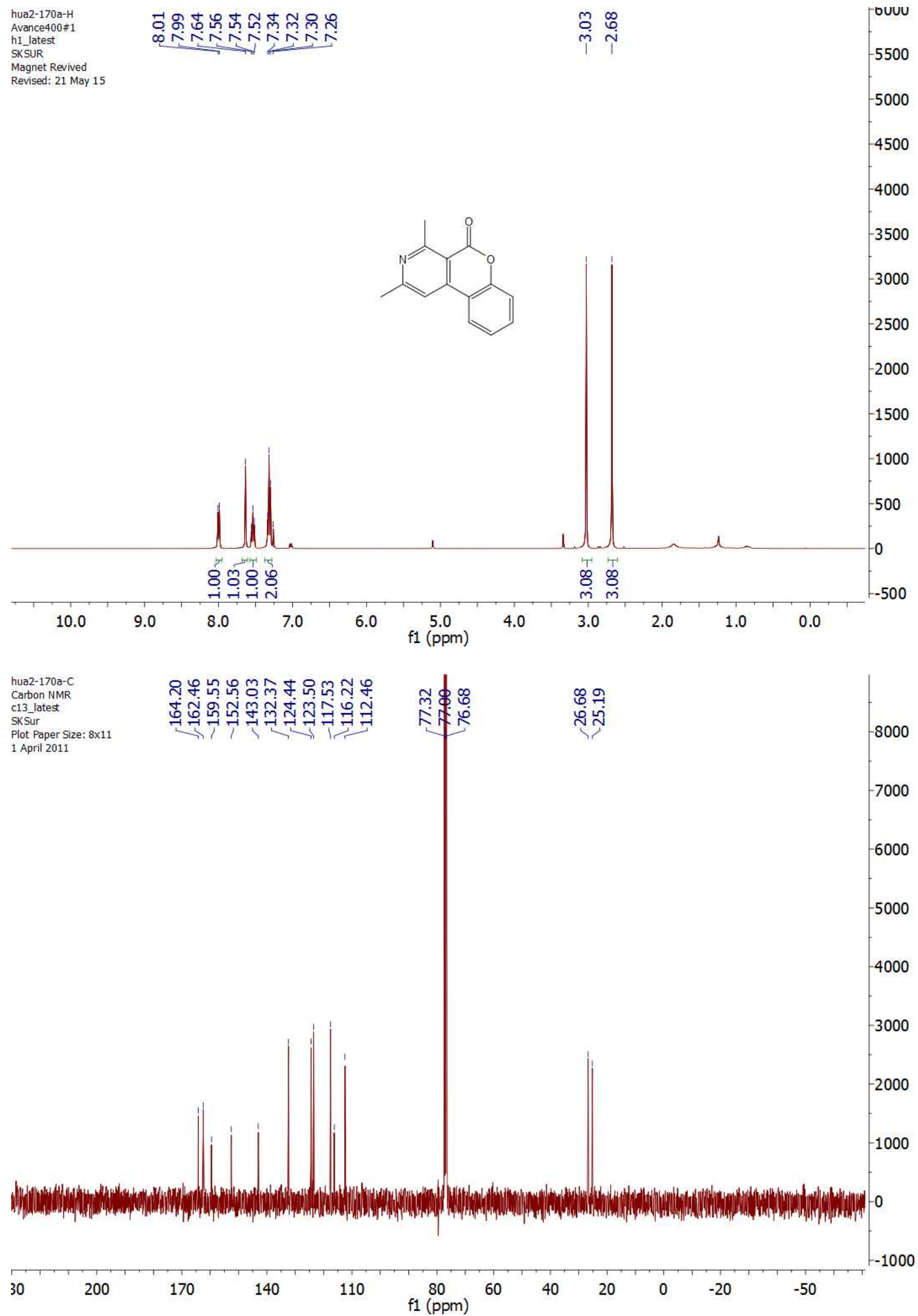